Florida International University

FIU Digital Commons

FIU Electronic Theses and Dissertations

University Graduate School

$11-6-2013$

\title{
The Utilization of Chiral Ion Mobility Spectrometry for the Detection of Enantiomeric Mixtures and Thermally Labile Compounds
}

Howard K. Holness

Florida International University, holnessh@fiu.edu

DOI: $10.25148 /$ etd.FI13120421

Follow this and additional works at: https://digitalcommons.fiu.edu/etd

Part of the Analytical Chemistry Commons, Evidence Commons, Laboratory and Basic Science Research Commons, and the Science and Technology Law Commons

\section{Recommended Citation}

Holness, Howard K., "The Utilization of Chiral Ion Mobility Spectrometry for the Detection of Enantiomeric Mixtures and Thermally Labile Compounds" (2013). FIU Electronic Theses and Dissertations. 988.

https://digitalcommons.fiu.edu/etd/988

This work is brought to you for free and open access by the University Graduate School at FIU Digital Commons. It has been accepted for inclusion in FIU Electronic Theses and Dissertations by an authorized administrator of FIU Digital Commons. For more information, please contact dcc@fiu.edu. 


\title{
FLORIDA INTERNATIONAL UNIVERSITY \\ Miami, Florida
}

THE UTILIZATION OF CHIRAL ION MOBILITY SPECTROMETRY FOR THE DETECTION OF ENANTIOMERIC MIXTURES AND THERMALLY LABILE COMPOUNDS

\author{
A dissertation submitted in partial fulfillment of \\ the requirements for the degree of \\ DOCTOR OF PHILOSOPHY \\ in \\ CHEMISTRY \\ by
}

Howard K. Holness 
To: Dean Kenneth G. Furton

College of Arts and Sciences

This dissertation, written by Howard K. Holness, and entitled The Utilization of Chiral Ion Mobility Spectrometry for the Detection of Enantiomeric Mixtures and Thermally Labile Compounds, having been approved in respect to style and intellectual content, is referred to you for judgment.

We have read this dissertation and recommend that it be approved.

Joong-Ho Moon

Norman D. H. Munroe

Piero Gardinali

Kenneth G. Furton

José R. Almirall, Major Professor

Date of Defense: November 6, 2013

The dissertation of Howard K. Holness is approved.

Dean Kenneth G. Furton

College of Arts and Sciences

Dean Lakshmi N. Reddi

University Graduate School

Florida International University, 2013 
(C) Copyright 2013 by Howard K. Holness

All rights reserved. 


\section{DEDICATION}

I dedicate this work to my wife, Davia, my parents Howard and Shyrel and last but certainly not least my little bambino, David! 


\section{ACKNOWLEDGMENTS}

I would like to acknowledge my dissertation committee for their guidance during my tenure as a chemistry graduate student. Special thanks goes to my major professor Dr. José Almirall, who has constantly pushed and demanded more of his students with the expectation that they produce the highest quality research, I believe those efforts have paid off. I would also like to acknowledge Dr. Furton, who has served on my dissertation committee and I have watched work tirelessly both as a researcher and administrator during my time at FIU. Both Drs. Almirall and Furton have made me appreciate the dedication and love required in leading successful research groups. This has been an unforgettable experience that has been unique to me and I believe should be ubiquitous to

all graduate students. To my lab group (A-Team), the Furton Females (and males) and all the friends I have made during this journey, thank you for being there. 


\begin{abstract}
THE UTILIZATION OF CHIRAL ION MOBILITY SPECTROMETRY FOR THE

DETECTION OF ENANTIOMERIC MIXTURES AND THERMALLY LABILE

COMPOUNDS

By
\end{abstract}

Howard K. Holness

Florida International University, 2013

Miami, Florida

Professor José R. Almirall, Major Professor

This dissertation utilized electrospray ion mobility mass spectrometry (ESI-IMS-MS) to develop methods necessary for the separation of chiral compounds of forensic interest. The compounds separated included ephedrines and pseudoephedrines, that occur as impurities in confiscated amphetamine type substances (ATS) in an effort to determine the origin of these substances. The ESI-IMS-MS technique proved to be faster and more cost effective than traditional chromatographic methods currently used to conduct chiral separations such as gas and liquid chromatography. Both mass spectrometric and computational analysis revealed the separation mechanism of these chiral interactions allowing for further development to separate other chiral compounds by IMS. Successful separation of chiral compounds was achieved utilizing a variety of modifiers injected into the IMS drift tube. It was found that the modifiers themselves did not need to be chiral in nature and that achiral modifiers were sufficient in performing the required separations. The ESI-IMS-MS technique was also used to detect thermally labile compounds which are commonly found in explosive substances. The methods developed provided mass 
spectrometric identification of the type of ionic species being detected from explosive analytes as well as the appropriate solvent that enhances detection of these analytes in either the negative or positive ion mode. An application of the developed technique was applied to the analysis of a variety of low explosive smokeless powder samples. It was found that the developed ESI-IMS-MS technique not only detected the components of the smokeless powders, but also provided data that allowed the classification of the analyzed smokeless powders by manufacturer or make. 


\section{TABLE OF CONTENT}

CHAPTER

PAGE

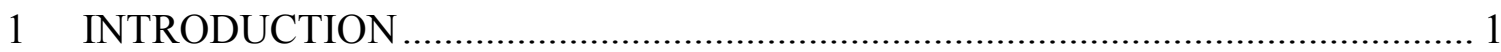

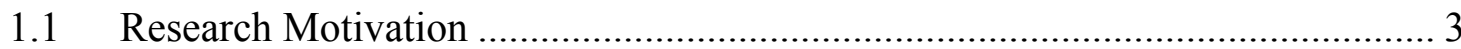

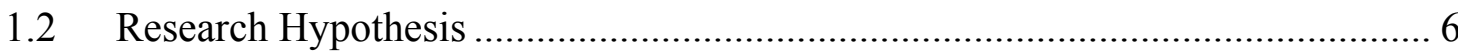

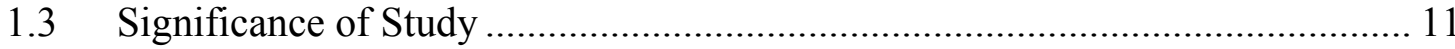

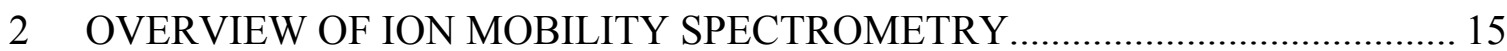

2.1 Theory of Electrospray Ionization............................................................. 18

2.2 Mechanics of the Electrospray Process: The Taylor Cone …………………... 22

2.3 History of Mass Spectrometry........................................................................ 25

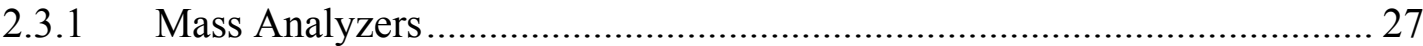

2.3.2 Coupling IMS to Mass Spectrometry ......................................................... 28

2.4 Performance of Electrospray Ionization versus Nickel-63 Ionization. .............. 32

2.5 Charge Competition in IMS ............................................................................ 41

2.6 Calculating Reduced Mobility in IMS experiments........................................... 46

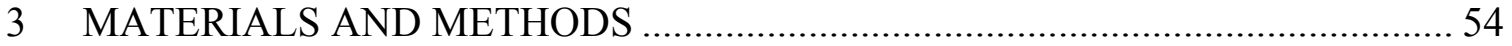

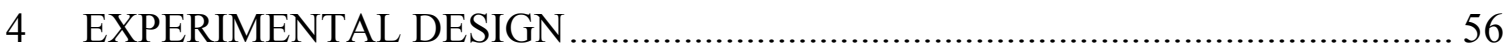

4.1 Adaptation of an Ion Mobility Spectrometer for Chiral Separations .................. 56

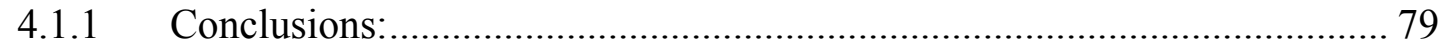

4.2 Utilization of an Electrospray Ionization Ion Mobility Spectrometer for Chiral

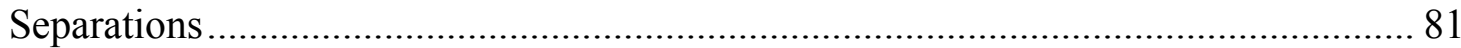

4.2.1 Optimization of the Electrospray Ionization Source................................... 86

4.2.2 Optimization of the Drift Gas Flow and Tube Potential............................. 89

4.2.3 Utilizing a Quadrupole Mass Spectrometer as an IMS detector................. 93

4.2.4 Analysis of Multiple Component Mixtures Utilizing ESI-IMS-MS............ 98

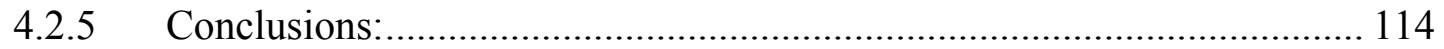

4.3 Application of Appropriate solvent chemistry for the Chiral analysis of

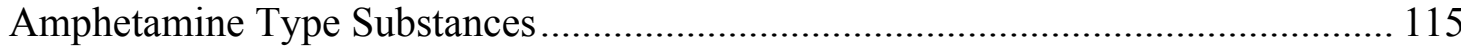

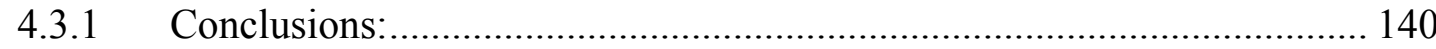

4.4 Application of Chiral Separations to Samples ………..................................... 141

4.4.1 Conclusions:...................................................................................... 149

4.5 Development of a Suitable IMS methods for the detection of Thermally Labile

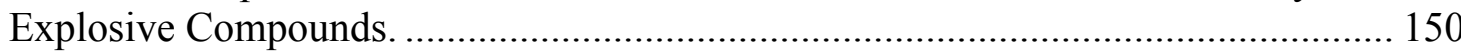

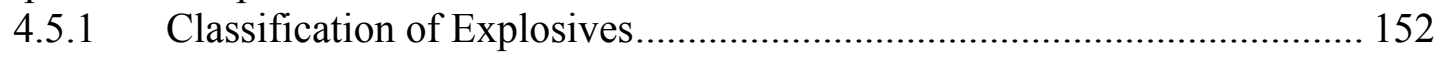

4.5.2 Analysis of Smokeless Powder Samples ……………………................ 165

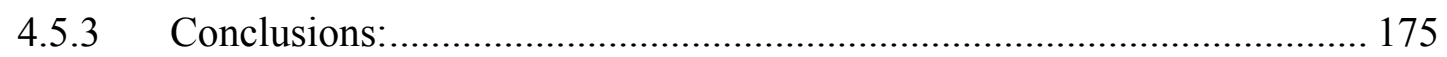

4.6 Detection and Mass Spectrometric Characterization of explosive analytes using ESI-IMS-MS 176 


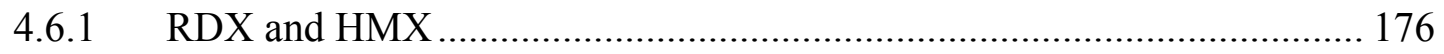

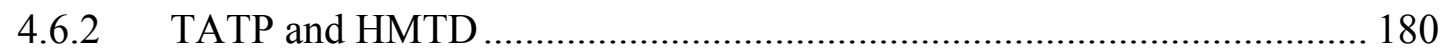

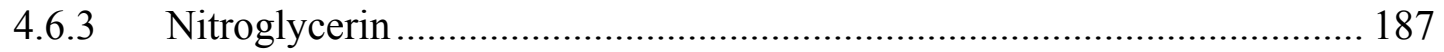

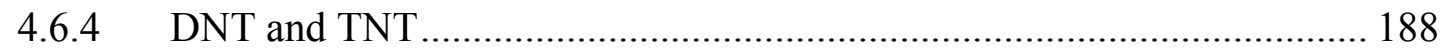

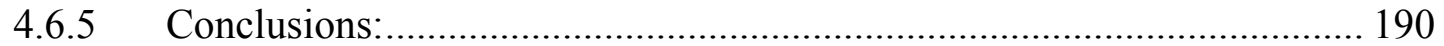

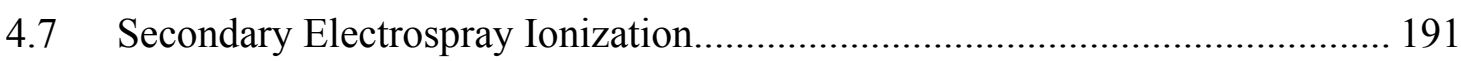

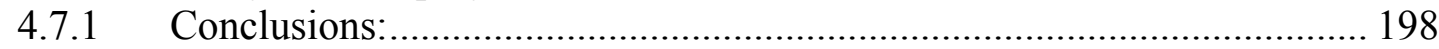

5 OVERALL CONCLUSIONS AND DISCUSSION ..Error! Bookmark not defined. 5.1 Utilization of Ion Mobility for Chiral Separation .............................................. 199

5.2 Application of Appropriate Solvent System for Chiral Analysis..................... 201

5.3 Development of IMS methods for the detection of Thermally Labile Explosive

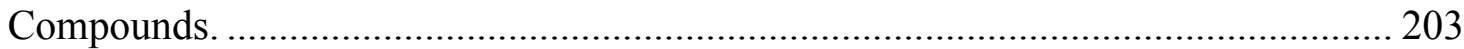

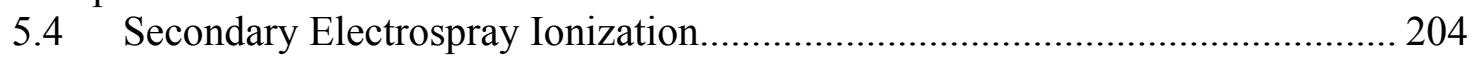

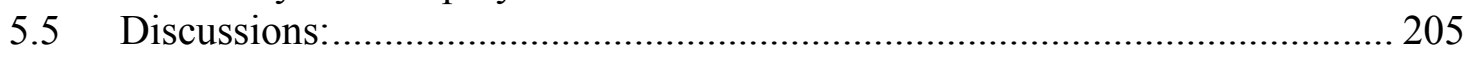

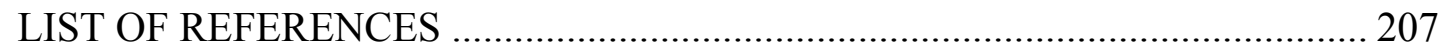

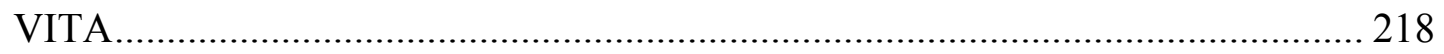




\section{LIST OF TABLES}

TABLE

PAGE

Table 1: Drift Times of ATS analyzed on Ionscan 400B .......................................... 70

Table 2: Effect of Modifier on Drift time of Analytes................................................. 78

Table 3: Masses of Polyethylene glycol (PEG) detected by Mass Spectrometer ............ 95

Table 4: Ionization energies of common ESI solvents and analytes. .......................... 100

Table 5: Full width half maximum (FWHM) of peaks obtained with different solvent systems of analytes at 50ng/ $\mu \mathrm{L}$ on ESI-IMS-MS .................................................. 102

Table 6: Observed drift times and reduced mobility of target analytes ........................ 118

Table 7: Effect of modifier on resolution between (R,S)-ephedrine and (S,S)-

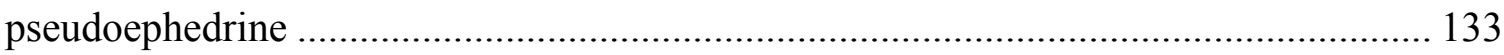

Table 8: Physical properties of modifiers used for chiral separations [129] ................. 134

Table 9: Explosive Compounds and Classification ................................................ 151

Table 10: Non-energetic materials found in Low Explosive smokeless powders .......... 155

Table 11: Results from ESI-IMS-MS analysis of Smokeless powder extracts in positive

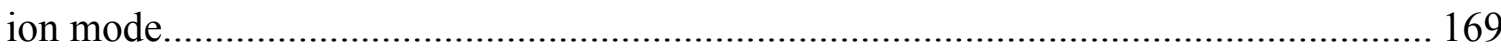

Table 12: Results from ESI-IMS-MS analysis of Smokeless powder extracts in negative

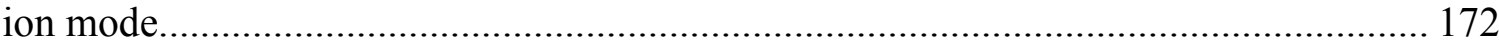




\section{LIST OF FIGURES}

FIGURE

PAGE

Figure 1: Starting materials used to make methamphetamine [11] …............................ 4

Figure 2: Diagram of applied voltage gradient in Ion Mobility Drift Tube....................... 8

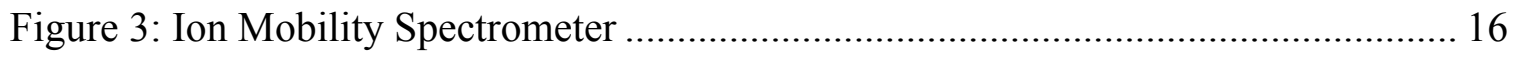

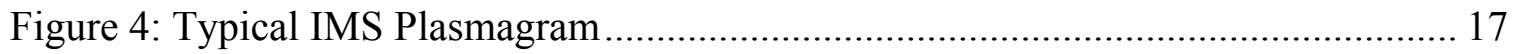

Figure 5: Illustration of ESI as a chemical Redox reaction cell [46] ............................ 18

Figure 6: Dispersion of a droplet formed under nanoESIspray conditions [47] .............. 20

Figure 7: Spherical drop formed at ESI tip under no electric field $(0 \mathrm{KV})$..................... 23

Figure 8: Elongated drop formed at ESI Tip under strong electric field $(3.5 \mathrm{KV}) \ldots \ldots \ldots \ldots . .23$

Figure 9: Image showing continuous flow of analyte onto field desorption sorbent....... 25

Figure 10: Improvement in signal to noise $(\mathrm{S} / \mathrm{N})$ obtained by using IMS coupled to MS

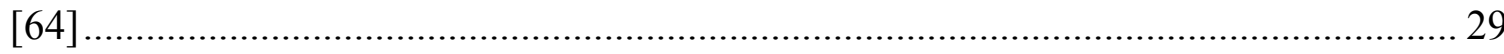

Figure 11: Micromachined Fused Silica tips used in ESI sources [68] ......................... 37

Figure 12: Plot of Ionization efficiency versus ESI flow rate [77] .............................. 37

Figure 13: Plot of Ion current with ESI flow rate [77] ......................................... 38

Figure 14: ESI-IMS interface showing solvent being sprayed into IMS [77] ................ 39

Figure 15: Effect of drift tube temperature on peak resolving power in ESI-IMS at; A-

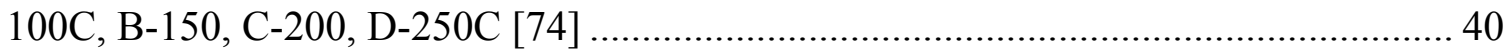

Figure 16: Ionization of Cocaine using Nickel 63 source......................................... 41

Figure 17: Ionization of Cocaine using Electrospray Ionization ................................. 42

Figure 18: Hypothetical example of selecting dopant to eliminate unwanted peaks [1].. 43

Figure 19: Various ion mobility spectra showing interferences from surrounding

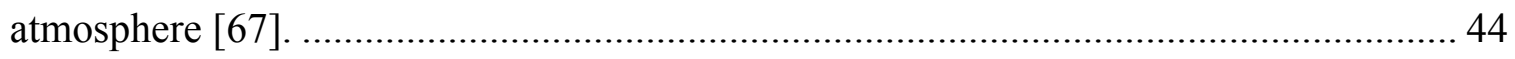

Figure 20: Alpha vs. Electric field for a group of analytes in differential mobility [93].. 51 
Figure 21: Effect of temperature on calculated reduced mobility values of four

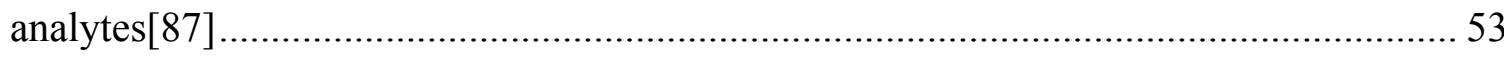

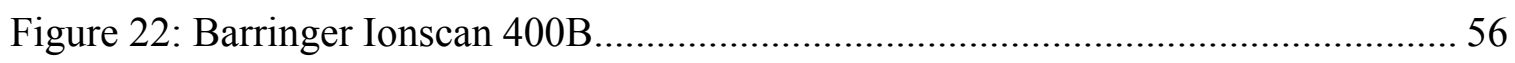

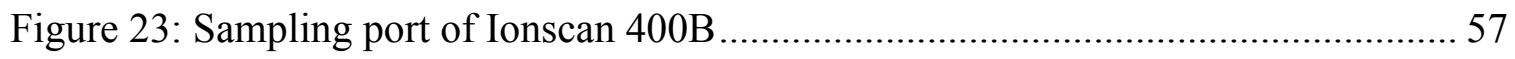

Figure 24: Location of Dopant on Ionscan 400B.......................................................... 58

Figure 25: Comparison of RIP with (a) Nicotinamide and (b) Air.................................. 60

Figure 26: Gravimetric determination of S-2-Butanol permeation rate............................ 62

Figure 27: Image showing external dopant tube used for S-2-Butanol permeation ......... 62

Figure 28: Peak obtained from S-2-Butanol permeation .................................................. 63

Figure 29: Images showing arrangement for direct infusion of modifier on Ionscan 400B 64

Figure 30: Ionscan 400B analysis of D and L methionine without modifier.................... 65

Figure 31: Ionscan 400B analysis of D and L methionine using S-2-Butanol modifier... 66

Figure 32: Improved chiral separation of D and L methionine on Ionscan 400B using S-2Butanol modifier.

Figure 33: Plasmagram of Amphetamine Type Substances (ATS) analyzed by Ionscan400B 68

Figure 34: Plasmagrams of ATS analyzed by GE Itemiser 2 showing only the detection of Methamphetamine and cathinone, ephedrine and pseudoephedrine cannot be detected.. 69

Figure 35: Diazepam and Cocaine analyzed on Ionscan 400B with 0.2ms Gate Pulse.... 71

Figure 36: Diazepam and Cocaine analyzed on Ionscan 400B with $0.05 \mathrm{~ms}$ Gate Pulse showing improved resolving power............................................................................... 72

Figure 37: Effect of Gate pulse on resolution between Diazepam and Cocaine .............. 73

Figure 38: Ion intensity vs. Gate Pulse duration on Ionscan 400B.................................. 74

Figure 39: Separation of select ATS on Ionscan 400B ……………………………...... 75

Figure 40: Chiral separation of select ATS using S-2-Butanol at 500uL per hour infusion 
Figure 41: Effect of Modifier infusion rate on drift time separation of analytes on Ionscan 400B. 77

Figure 42: Effect of Modifier on drift time of Chiral Analytes ................................... 78

Figure 43: Effect of modifier on drift time of Cocaine and Diazepam........................... 79

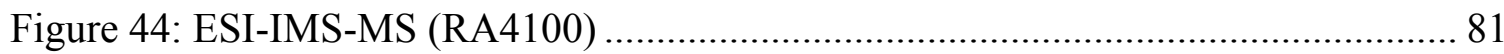

Figure 45: Cooled Electrospray Ionization Source on RA4100 …............................. 82

Figure 46: High Voltage Power module for RA4100 ................................................. 83

Figure 47: (a) 3mm Hole in Faraday detector of IMS and (b) $200 \mu \mathrm{m}$ Orifice of MS inlet

Figure 48: (a) IMS and (b) Single ion plot of Diphenylamine showing the mobility of the

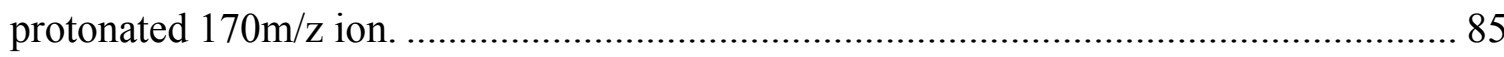

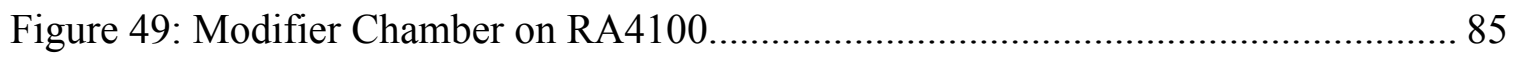

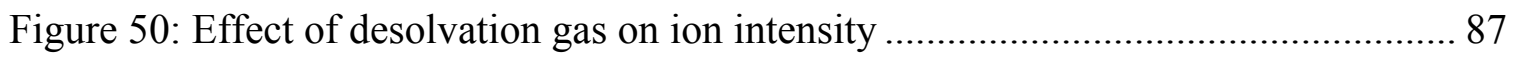

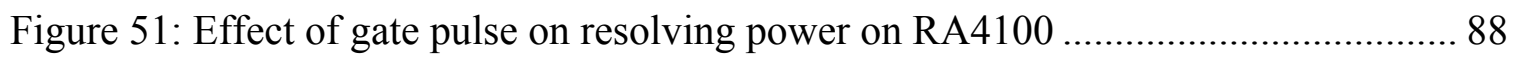

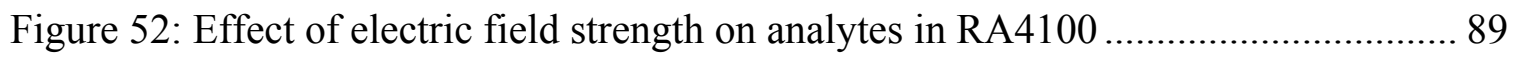

Figure 53: Effect of Electric field strength on ion intensity ..................................... 90

Figure 54: Effect of RA4100 drift gas flow rate on ion mobility of a mixture of analytes

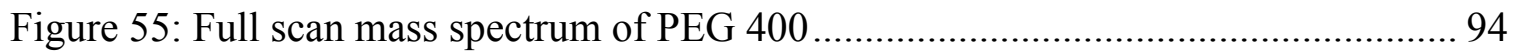

Figure 56: Repeating unit of Polyethylene glycol (PEG) 400 polymer observed on mass

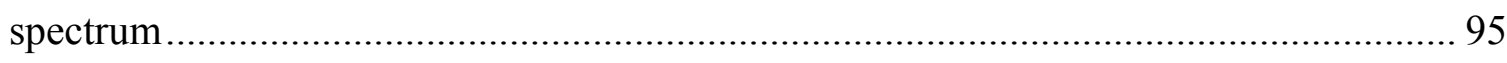

Figure 57: Overlay of Ion Mobility and Single ion monitoring of PEG 400 on RA4100 96

Figure 58: Mass spectrum of Cocaine obtained on RA4100 showing inaccurate mass

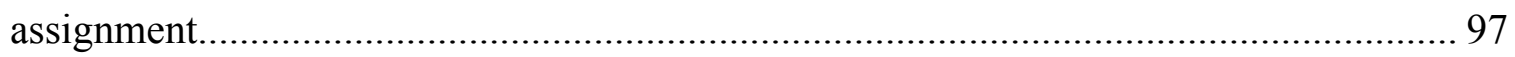

Figure 59: Correlation of observed versus actual mass of analytes on calibrated RA4100 98

Figure 60: Effect of acid strength on detection of analytes 100 
Figure 61: 3-D plot of solvent effect on analyte intensity 101

Figure 62: Species observed from ESI-IMS-MS of methanol:water mixture with 2.5\% formic acid showing Ion Mobility and Mass Spectrum in Positive Ion Mode 104

Figure 63: Species observed from ESI-IMS-MS of methanol:water with 10mMol ammonium nitrate and 2.5\% formic acid showing Ion Mobility and Mass Spectrum in Positive Ion Mode 105

Figure 64: Response of Cocaine in 0.1\% Formic acid............................................ 106

Figure 65: Response of Cocaine in methanol:water containing 5\% Formic acid.......... 107

Figure 66: Species observed from ESI-IMS-MS of methanol:water with $10 \mathrm{mMol}$ ammonium nitrate showing Ion Mobility and Mass Spectrum in Negative Ion Mode 108

Figure 67: Mass Spectra of RDX species formed in (a) sodium chloride based solvent, (b) methanol:water only and (c) ammonium nitrate based solvent

Figure 68: Species observed from ESI-IMS-MS of 4-Nitro-diphenylamine dissolved in methanol:water with $0.1 \%$ chloroform by (a) Ion mobility in negative ion mode and (b) Full scan mass spectrum in negative ion mode.

Figure 69: Effect of chloroform on ion intensity for negative mode analysis showing enhanced detection of 2,4 Dinitrotoluene

Figure 70: Analysis of 50ppm Caffeine, R-Methamphetamine, SR-Ephedrine, Diazepam and Cocaine on RA4100 using both $2.5 \%$ formic acid and $0.1 \%$ formic acid 116

Figure 71: Single Ion monitoring plot of molecular ions for a multicomponent mixture

Figure 72: Overlay of target analytes on ESI-IMS-MS (RA4100) ............................... 118

Figure 73: Effect of Modifier Infusion rate on analyte drift time............................... 119

Figure 74: Effect of Modifier infusion rate on drift time separation of analytes on RA4100

Figure 75: Effect of Modifier Gas flow on drift time of analytes

Figure 76: Mass Spectrum of SS-Pseudoephedrine on RA4100 122

Figure 77: Single Ion Monitoring of ions from SS-Pseudoephedrine on RA4100 123 
Figure 78: Mass spectrum of SS-Pseudoephedrine on RA4100 with S-2-Butanol modifier infusion

Figure 79: Separation of chiral compounds using achiral modifier 2-butanol

Figure 80: Resolution obtained between ephedrine and pseudoephedrine using achiral modifiers 126

Figure 81: Adaptation of Pirkle interaction between chiral analyte and chiral modifier (a) having a weak interaction and (b) having a strong interaction.

Figure 82: Representation of analyte-cluster formation in the drift tube region of IMS with modifier (Not drawn to scale, IMS gate not shown for clarity).

Figure 83: Mass spectrum of analyte (RS-ephedrine) with modifier clusters (ethanol). 130

Figure 84: Single Ion monitoring of ions from analyte modifier interaction

Figure 85: Newman and visual projections of (R,S)-ephedrine with two n-octanol modifiers depicting the "bridged" motif or compact configuration.

Figure 86: Newman and visual projections of (S,S)-pseudoephedrine with two n-octanol modifiers depicting the "independent" motif or expanded confirmation 135

Figure 87: Separation between ephedrine and pseudoephedrine using n-octanol modifier

Figure 88: Analysis of ephedrine and pseudoephedrine without the use of modifier .... 137

Figure 89: Ratio of chiral impurities found in ATS showing three different groups of origin A,B and C as determined by Capillary Electrophoresis [25].

Figure 90: Differences in structure for target chiral compound ephedrine and internal standard cocaine.

Figure 91: Analysis of chiral compounds with cocaine internal standard on RA4100 .. 145

Figure 92: Drift time of analytes relative to cocaine internal standard utilizing various modifiers

Figure 93: Reduced mobility values for chiral compounds utilizing various modifiers. 147 Figure 94: Analysis of a mixture of analytes using both chiral and non-chiral analysis on RA4100 
Figure 95: Determination of ephedrine and pseudoephedrine ratio from a single mixture

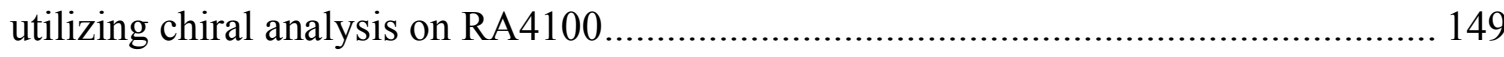

Figure 96: World Incident Map of Explosive Use (www.globalincidentmap.com) ....... 153

Figure 97: Analysis of DEP, DPA, MC and EC at $170^{\circ} \mathrm{C}$ in positive in mode .............. 159

Figure 98: Analysis of DEP, DPA, MC and EC at $110^{\circ} \mathrm{C}$ in positive in mode, showing inability to detect DPA, MC and $\mathrm{EC}$ at this temperature............................................... 160

Figure 99: Analysis of DEP, DPA, MC and EC at $150 \mathrm{C}$ in positive in mode showing the detection of all analytes with good separation.

Figure 100: Single Ion Monitoring of molecular ions of DEP, DPA, MC and EC at 150C in positive in mode

Figure 101: Comparison of Standards DNT, NG, 2-NDPA and 4-NDPA in negative mode using Air vs. Nitrogen drift gas 163

Figure 102: Single Ion Monitoring of ions of DNT, NG, 2-NDPA and 4-NDPA at $150^{\circ} \mathrm{C}$ in negative in mode. 164

Figure 103: Extraction scheme used for Smokeless powder analysis by ESI-IMS-MS. 166 Figure 104: GC-ECD response of smokeless powder extracts with varying extraction time. 167

Figure 105: Analysis of smokeless powder extracts in positive ion mode 168

Figure 106: Dendrogram showing clustering of smokeless powders based on analysis by ESI-IMS-MS in positive ion mode.

Figure 107: Dendrogram showing clustering of smokeless powders based on analysis by ESI-IMS-MS in negative ion mode. 173

Figure 108: Analysis of Green dot in negative ion mode 174

Figure 109: Analysis of Green dot in the positive ion mode ……………...................... 174

Figure 110: Molecular structure of (a) RDX and (b) HMX......................................... 177

Figure 111: $200 \mathrm{ng} / \mu \mathrm{L}$ of $\mathrm{RDX}$ in MeOH:Water analyzed in the negative ion mode. ... 178

Figure 112: Thermodynamically favorable adduct of RDX not observed in ESI-IMS-MS analysis in negative mode. 
Figure 113: Expected HMX adduct not observed on ESI-IMS-MS in negative ion mode 179

Figure 114: Molecular structure of (a) TATP and (b) HMTD........................................ 181

Figure 115: Results from the analysis of TATP in negative ion mode.......................... 182

Figure 116: Single Ion Monitoring of TATP ion fragment obtained in the negative ion mode. 183

Figure 117: Overlay of 20ppm TATP and 20ppm HMTD detected in positive ion mode 184

Figure 118: ESI-IMS-MS of (a) HMTD and (b) TATP in positive ion mode using Methanol:Water solvent with $2.5 \%$ formic acid 185

Figure 119: ESI-IMS-MS of (a) HMTD and (b) TATP in positive ion mode using Methanol:Water solvent with $2.5 \%$ formic acid with $10 \mathrm{mMol}$ Ammonium Nitrate 186

Figure 120: Structure of Nitroglycerin 187

Figure 121: ESI-IMS-Mass Spectrum of Nitroglycerin dissolved in MeOH:Water in the negative ion mode. 188

Figure 122: Structure of (a) 2,4 Dinitrotoluene and (b) 2,4,6 Trinitrotoluene. 189

Figure 123: (a) Ionization of Nitro-aromatics in Methanol:Water solution and (b) Suppression of Nitro-aromatics in Nitrate solution 190

Figure 124: Modifications to allow secondary electrospray ionization on RA4100 with SPME fiber introduction 193

Figure 125: (a) Ion mobility and (b) Mass Spectrum of Secondary ESI of 2,4 DNT collected by SPME Headspace 194

Figure 126: GC-FID data showing amount of DNT extracted by Headspace SPME .... 195

Figure 127: Response of DNT on GC-FID .......................................................... 196

Figure 128: Headspace extraction of DNT by Secondary ESI on RA4100.................... 197

Figure 129: Model of Secondary ESI as it occurs within the RA4100 .......................... 198 


\section{LIST OF EQUATIONS}

EQUATION

PAGE

Equation 1: Relationship of ion drift velocity to electric field strength ............................ 8

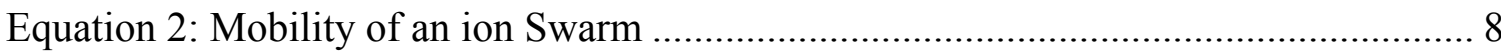

Equation 3: Probable redox reactions occurring during electrospray ionization in positive

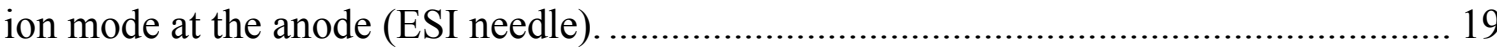

Equation 4: Relationship of voltage and shape of a drop that determines the ESI Taylor cone.

Equation 5: Ionization of air molecules by nickel beta particles ...................................... 33

Equation 6: Including mobility constant to correlate ion velocity and electric field strength [1]

Equation 7: Rearrangement of mobility constant to include the length of the drift tube.. 46

Equation 8: Relating reduced mobility to mobility [1] ..................................................... 47

Equation 9: Expanded reduce mobility equation [1] ..................................................... 47

Equation 10: Relationship between reduced mobility values of two analytes [1;87]...... 47

Equation 11: Expanded ion mobility equation [1] ......................................................... 49

Equation 12: Relationship between field condition, field strength and gas density [1] ... 49

Equation 13: Coefficient of mobility in a high electric field [92] ……........................... 50

Equation 14 Proton abstraction in negative mode ESI [115]........................................ 110

Equation 15 Anion attachment in negative mode ESI [115] ......................................... 111

Equation 16: Typical combustion reaction between a fuel and oxygen ......................... 158

Equation 17: Combustion reaction of nitroglycerin during detonation .......................... 158 


\section{LIST OF ACRONYMS AND ABBREVIATIONS}

\begin{tabular}{ll} 
AMU & Atomic Mass Unit \\
APCI & Atmospheric Pressure Chemical Ionization \\
CAS & Chemical Abstract Service \\
CE & Capillary Electrophoresis \\
DEP & Diethyl phthalate \\
DNT & Dinitrotoluene \\
DPA & Diphenylamine \\
EC & Ethylcentralite \\
ECD & Electron Capture Detector \\
ESI & Electrospray Ionization \\
FAB & Fast Atom Bombardment \\
FID & Flame Ionization Detection \\
GC & Gas Chromatography \\
HMTD & Hexamethylene triperoxide \\
HMX & High Molecular Weight RDX \\
IMS & Ion Mobility Spectrometry \\
LC & Liquid Chromatography \\
LD & Laser Desorption \\
MAGIC & Mono disperse aerosol generating interface for chromatography \\
MC & Methylcentralite \\
MS & Mass Spectrometry \\
NDPA & Nitro-dihphenylamine \\
NG & Nitroglycerin \\
OTS & Off the Shelf \\
RDX & Research Development Explosive \\
SPME & Solid Phase Microextraction \\
TATP & Triacetone Triperoxide \\
TNT & Trinitrotoluene \\
TOF & Time of Flight \\
& \\
\hline
\end{tabular}




\section{INTRODUCTION}

Ion mobility spectrometry (IMS) has been described throughout scientific literature as both a stand-alone separation technique and as a hyphenated technique used to enhance other analytical instruments [1]. Despite this flexibility and versatility, the applications of IMS have not grown as quickly as that of gas chromatography (GC) or liquid chromatography (LC) even though IMS has similar, if not greater resolving capabilities than that of the afore- mentioned techniques [2]. The lack of growth may be attributed to IMS' niche use for military and security applications where its high speed, low cost and simple operation have simultaneously made it both robust for field use and also made it less attractive for more advanced analytical applications within laboratories within academia. My dissertation describes the use of IMS as a lab-based analytical technique able to perform separations on par with GC and LC applications. Ion mobility spectrometry, in its present form was developed in the state of Florida during the 1970s and was previously referred to as plasma chromatography [1;3-5]. The technique is more closely related to capillary electrophoresis (CE) than either GC or LC as it utilizes similar separation principles to $\mathrm{CE}$, where charged analytes are moved under the influence of an applied electric field. Analytes that have a smaller mass-to-charge ratio migrate faster than analytes with a larger mass-to-charge ratio, thereby resulting in separation of the mixture. However, unlike capillary electrophoresis, IMS does not require a solvent for separation to occur, instead analytes are separated in the gas phase under atmospheric conditions much faster (on the order of milliseconds) than CE, LC or GC which normally require at least several minutes to complete the separation. The speed of IMS has driven 
those applications that take advantage of both the speed of analysis and the resulting lower cost of analysis. Ion mobility spectrometry is not only found in niche applications involving civilian and military requirements, but also pharmaceutical, aeronautical, agricultural and petrochemical industries alike [6;7]. Analytical chemists within academia have been reluctant to adopt Ion Mobility as a separation because of the ubiquitous presence of GC and LC separation systems. These systems, though much more costly than IMS, offer improved separation efficiency for many applications at the expense of lengthy separation/analysis times. Compounding the reluctance of academic use is also the fact that separations within the realm of academia do not require the high speed separations offered by IMS, unlike separations that are required within industry or for security applications. These factors make the speed advantage offered by IMS of little value to academia. What has allowed IMS to survive within academia under such neglect is its relatively simple operation and low acquisition costs. Many research groups willing to perform ion mobility experiments construct their own instruments from easily accessible and relatively inexpensive components [8]. The low acquisition and operating costs have resulted in a small but strong network of IMS research groups that have been able to develop analytical methods for analytes which previously proved difficult or, in some cases, impossible to analyze utilizing traditional chromatographic techniques such as GC and LC. Some of these groups have no chromatographic equipment available nor do they have the need for such equipment, because of the versatility of IMS. The question then still remains, why, if IMS has been so readily accepted within industry and craved by some within academia, has it not been more popular? The answer appears to stem from the fact that traditional IMS has the stigma of being only used as a front end 
technique, requiring hyphenation with other techniques in order to be truly useful. My body of work will however show the capabilities of IMS as a standalone separation technique, particularly for the use in chiral separations. The present work will also highlight the enhancements IMS offers to techniques such as Mass Spectrometry, ultimately showcasing the symbiotic relationship IMS creates when used at its full potential.

\subsection{Research Motivation}

The current research project was motivated from the necessity to perform rapid and inexpensive analyses on substances commonly encountered by law enforcement, in particular analysis of controlled amphetamine type substances and explosives, some of which are thermally labile and some of which are chiral molecules. The current state of the art for the analysis of chiral molecules remains liquid chromatography utilizing a chiral stationary phase [9]. However, the ever growing variety and expense of chiral stationary phases appears to have stalled the progression of chiral analysis. The time required and expense to develop chiral separation methods utilizing liquid chromatography have been prohibitive and as a result, such analyses are not routinely performed within a crime lab, thereby limiting the amount of information generated by a particular laboratory. In the case of thermally labile compounds, these analyses are performed with instruments that have inherently poor detection limits for the target analytes of interest. The use of these instruments results in an inability to detect analytes when they are present and this missed detection, called a false negative, is one of the single greatest problems faced by the field of forensic science. The occurrence and 
subsequent elimination of false negatives is a powerful driving force in instrumental development within forensic science and the current research endeavor. The solutions to both these problems are quite different but I propose the use and development of a single analytical instrument, Ion Mobility Spectrometry (IMS) to solve both problems of interest. For chiral analysis, IMS offers the unique capability to combine its inherent high speed and low cost of operation to offset the high barriers to analysis normally encountered when conducting traditional LC-based chiral analysis. Today, the vast majority of pharmaceutical drugs sold within the United States are chiral in nature, meaning that these drugs, when analyzed by chiral separation techniques can yield law enforcement with valuable information as to their production, purity and origin [10]. The widely abused and purely synthetic amphetamine type substances (ATS) fall into this drug category. These drugs are synthesized from a variety of starting materials from remote clandestine laboratories before being transported and sold in different locations around the world. The most popular controlled ATS, S-methamphetamine, may be synthesized from one of two starting materials as shown in Figure 1.

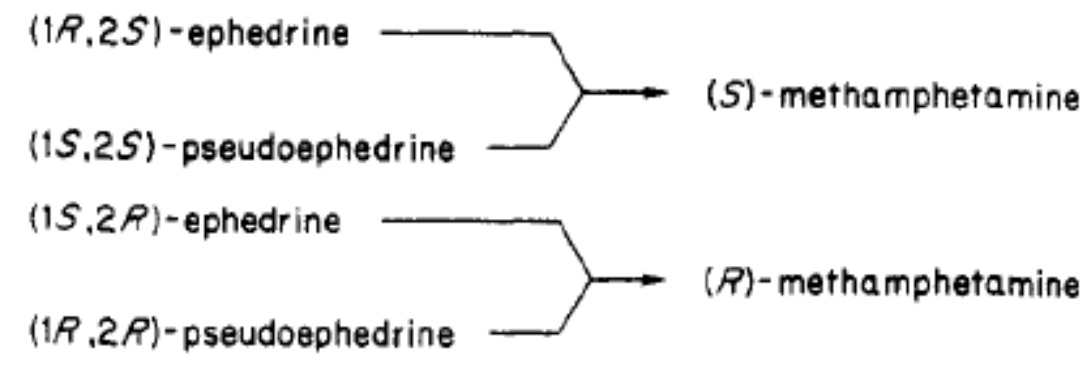

Figure 1: Starting materials used to make methamphetamine [11]

Analysis of these starting materials, $(\mathrm{R}, \mathrm{S})$ ephedrine and $(\mathrm{S}, \mathrm{S})$ pseudoephedrine, has been shown to provide additional information to law enforcement on the confiscated drug's 
origin and can also provide the ability to link different batches of confiscated drugs. The ability to analyze these substances once confiscated on a routine basis will provide a necessary investigative tool to law enforcement on their origin and also provide the ability to link different batches of seized substances solely on the basis of their chiral makeup. However, this information may only be obtained by utilizing chiral separation techniques. Chiral information has already proved useful when utilizing other analytical techniques such as liquid chromatography and capillary electrophoresis [11]. But, as previously stated, the high cost of chiral separation phases required to perform these analyses, coupled with laborious trial and error to develop a fully functioning technique has made these analyses too expensive to become routine. Ion mobility spectrometry has demonstrated the ability to perform chiral separations in a fraction of the time and at a fraction of the cost of traditional chiral separation techniques [12-15]. Ion mobility spectrometry has also demonstrated the ability to be more multifaceted by providing capabilities to conduct a wide array of other analysis in addition to chiral separations without the requirement of specialized instrument parts and consumables [6;7]. As a result, IMS may be viewed as the ideal tool by which a routine chiral separation method for ATS may be developed for the use in current forensic laboratories.

Thermally-labile compounds are those which are easily destroyed by heat. Compounds found in explosive materials often have this characteristic, therefore if these materials are subjected to typical analysis by gas chromatography they are often destroyed through thermal decomposition [16]. The very nature of gas chromatography, which utilizes heat to maintain analytes in a volatile state and drive them through the separation phase, may decompose some analytes to the point where they can no longer be detected. The IMS 
analysis allows for the detection of these substances without the requirement of extremely high temperatures that will cause their degradation. The lower temperatures allows the availability of more intact molecules for analysis, providing superior detection capabilities to that currently utilized. Presently, similar detection capabilities with these thermally labile substances can be obtained utilizing LC, however, the cost of LC instrumentation coupled with the cost of consumables makes analysis of these analytes less routine within the typical forensic laboratory.

\subsection{Research Hypothesis}

The research being described within my dissertation is derived from the fact that target analytes may be converted to gas phase ions from both volatile and non-volatile analytes. The conversion of analytes to gas phase ions will allow for rapid, low cost analysis of forensic samples of interest. Once gas phase ions are generated, the ions can then be separated using an applied electric field under ambient conditions on the basis of differences in shape to charge ratios. The interaction of these gaseous ions with the supporting atmosphere, referred to as drift gas, coupled with the differences in their shape to charge ratios, will cause these ions to migrate at different rates while in the applied electric field referred to as Ion Mobility Spectrometry (IMS). The difference in shape to charge will be used to separate chiral molecules using modifiers in the drift tube. The use of modifiers to conduct chiral separations in the gas phase is more cost effective than the current practice of purchasing a dedicated chiral separation phase as is the with GC and LC applications. Contrary to current literature, the modifiers required to conduct these gas phase separations are also chiral, however my work will show that these modifiers 
need not be chiral for separations to occur. The difference in migration rates will result in the separation of a mixture into its individual components, similar to the separation obtained from GC or LC but within a much shorter time frame, milliseconds versus minutes. The separation of these gas phase ions in IMS is carried out utilizing a short stainless steel tube typically $10 \mathrm{~cm}$ in length, which contains no stationary phase. As a result, the separation itself is not chromatography as there is no interaction between the analyte or mobile phase and a stationary phase as is the case with GC or LC. Ion mobility is more closely related to electrophoresis, where ions in a buffer solution are separated under the influence of an applied electric field. The fact that Ion Mobility is not true chromatography does result in it having slightly lower resolution capabilities than either GC or LC, but with much more rapid analysis times, lower operational costs and the ability to analyze both volatile and non-volatile analytes in a single technique, its benefits outweigh any shortcomings.

Prior to analysis of the sample by ion mobility, the sample itself must be ionized. A variety of sources may be used for ionization of samples, for these experiments, an electrospray ionization source (ESI) was used. A full description of that source is been given in the section on Theory of Electrospray Ionization (pg 18). Once the sample is ionized it is then pulsed into the separation region referred to as the drift tube. As previously mentioned, the drift tube is typically a $10 \mathrm{~cm}$ stainless steel tube which has differential voltages applied to either end. The tube itself is comprised of a series of stainless steel rings, each ring has a different applied voltage in a stepwise fashion to create a voltage gradient (Figure 2). The gradient is either positively or negatively biased to allow the separation of either positive or negatively charged gaseous ions. The ions 
created by the ionization source, referred to as an ion swarm, will drift at a rate proportional to the magnitude of the electric field as described by the following equation:

Equation 1: Relationship of ion drift velocity to electric field strength

$$
v_{d} \propto E
$$

Where $\mathrm{E}$ is the electric field strength and $\mathrm{v}_{\mathrm{d}}$ the drift velocity of the ion swarm [1]. For most analytes, the mobility $\mathrm{K}$, is different enough under an applied electric field to allow separation and individual characterization. The value for $\mathrm{K}$ may be further expanded to reveal its relationship between the temperature of the ion swarm and the average collision cross sectional area $(\Omega)$ of that ion swarm.

Equation 2: Mobility of an ion Swarm

$$
K=\left(\frac{3 q}{16 N}\right)\left(\frac{2 \pi}{\mu k T}\right)^{\frac{1}{2}} \frac{1}{\Omega_{D}}
$$
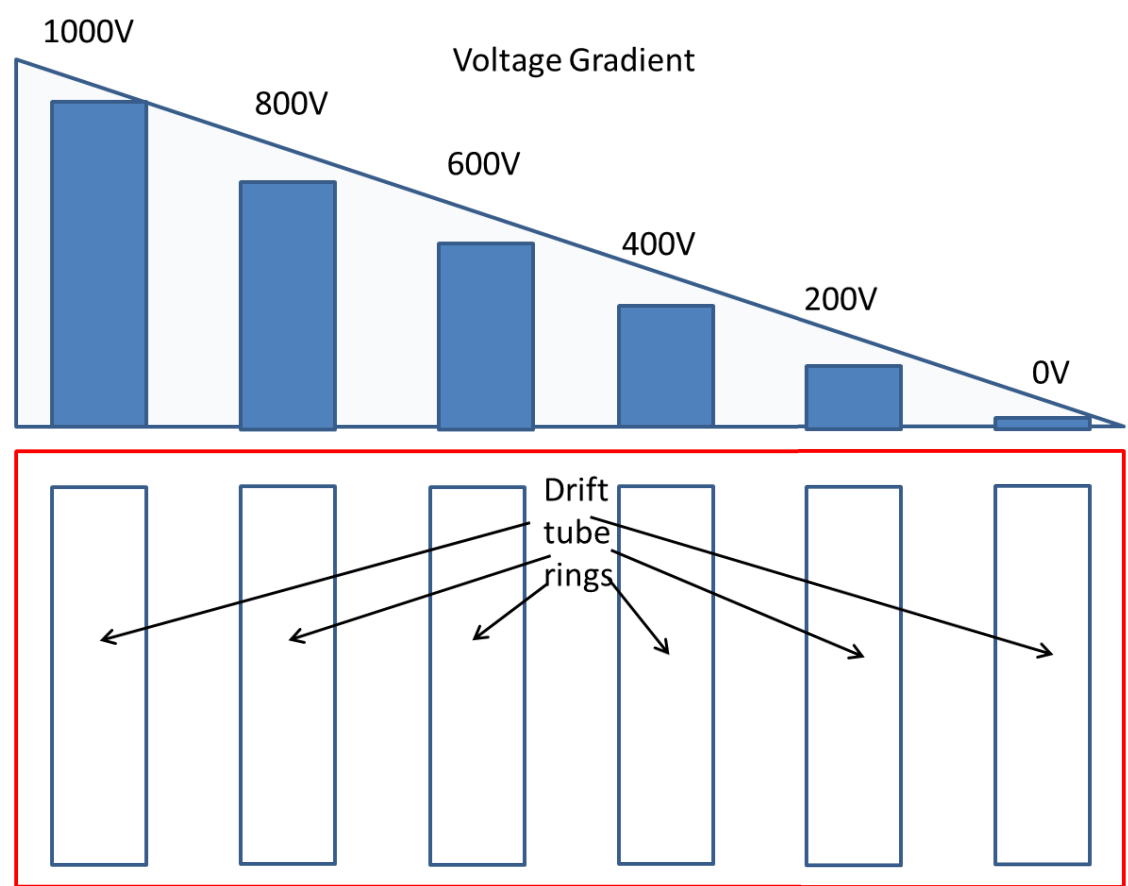

Drift Tube

Figure 2: Diagram of applied voltage gradient in Ion Mobility Drift Tube 
The ability to separate analytes on the basis of the cross sectional area allows Ion Mobility an additional degree of discrimination not found in many other separation techniques. The discrimination by shape to charge as opposed to only discriminating by mass to charge will be exploited throughout my study as I attempt to separate chiral compounds. The interaction of the analyte and supporting drift gas determines the level of separation. Higher molecular weight drift gases such as Carbon Dioxide or Argon, provides greater separation than do lower molecular weight drift gases such as helium and nitrogen. Other factors that affect the interaction of the drift gas with the ion swarm include the density of the drift gas $(N)$. An increase in drift gas density will result in an increase in collision frequency between the analyte and drift gas, again resulting in greater separation determined by greater shape to charge interaction [1].

As chiral compounds possess identical physical properties, including mass, they cannot be separated on the basis of mass-to-charge ratio. Mass spectrometry as a result is unable to discriminate these chiral compounds; however, these compounds often contain sufficient structural differences to be discriminated by their shape. For these experiments, the structural differences between chiral compounds will be amplified through the use of a modifier within the IMS drift gas. Previous work has revealed the ability of a drift gas modified with a chiral compound to preferentially interact with one chiral compound over another [12]. The aim of the present research is to build upon those experiments so that such separations can be conducted on amphetamine type substances (ATS) which are of significant forensic interest. The use of a modifier in Ion Mobility has a similar effect to the use of micelles in capillary electrophoresis, where the micelle or modifier is added in an attempt to enhance the separation capabilities of the technique. The modifier in this 
case is selected for its ability to preferentially interact with one chiral analyte more than another and thereby result in a separation of two optically active enantiomer pairs. Various modifiers were studied to investigate the degree of separation each produced as well as an investigation into the mechanism responsible for the separation. It was observed that the separation itself was ion-neutral based, as the modifier is introduced as a neutral species into the drift gas, while the analyte is ionized by the ionization source (ESI) to become a gaseous ion. Explicit details of these experiments and findings may be found in the section on Utilization of an Electrospray Ionization Ion Mobility Spectrometer for Chiral Separations (pg. 81). The process of ionizing and detecting thermally labile compounds utilizing ion mobility has been a common practice since the technique was developed. These compounds have eluded detection systems for years as a consequence of severe thermal degradation which rendered them invisible to various techniques. However, IMS has been able to not only detect these compounds, but do so with extreme efficiency. The ${ }^{63} \mathrm{Ni}$-based IMS systems have been reported to detect these compounds in the part per billion to part per trillion detection range [17]. The low detection limits result from the seemingly tailor-made ionization mechanism scheme between the radioactive nickel $\left({ }^{63} \mathrm{Ni}\right)($ see Equation 5) and many of these thermally labile species which contain electron receiving or electron withdrawing groups such as halides as nitrates. These groups are also responsible for the very unstable nature of these compounds, allowing them to be strong oxidizing agents as they interact with various fuels in an explosive chain. The explosive nature of thermally labile compounds is the single biggest reason for the importance of their detection in forensics. The misuse of these compounds has resulted in millions of dollars in property loss and thousands of 
injuries and loss of human life [18]. Ion mobility spectrometry allows for the intact detection of these analytes as well as their efficient detection when utilizing a nickel ionization source. But what these previous systems have lacked is the ability to perform simultaneous analysis of a mixture of these analytes. Traditionally, IMS units will sample a mixture of analytes and only the compound with the highest electron affinity will be ionized, leaving other analytes to be either not detected, or detected with limited response. The major drawback to using a traditional IMS system is that an interfering compound may be able to mask the presence of a target analyte from being detected. My project aims to alleviate this obstacle through the use of an ESI source rather than ${ }^{63} \mathrm{Ni}$ source. ESI, through the use of selected solvent systems, allows IMS to still be able to detect these thermally labile compounds but with the added advantage of ionizing most if not all analytes within a sample simultaneously. Electrospray ionization allows the technique to become truly analytical as opposed to being a preliminary screening tool. Various solvent systems will be tested and discussed to reveal the appropriate solvent makeup for both negative and positively ionized thermally labile species. The effects these solvent systems have on the type of ionic species generated and the effect of these solvent systems on the response for targeted analytes will also be thoroughly evaluated.

\subsection{Significance of Study}

My study has been undertaken to 1) improve the understanding of chiral separations in the gas phase and 2) provide scientists, including forensic scientists, with additional tools that will enhance the current separation and detection of amphetamine type substances and thermally labile compounds as well as provide new detection capabilities where none 
existed previously. The holistic forensic approach is designed in every experiment to ultimately be adopted for routine use within forensic crime laboratories. Experiments have been designed with cost and time factor considerations so that transitions from academia to practitioner are as seamless as possible. The first task of the present study which involves the separation of chiral compounds was derived from the growing need within the law enforcement community to analyze and categorize the increasingly large number of amphetamine type substances (ATS) being confiscated [19-21]. The ability of law enforcement to analyze and discriminate seized ATS will greatly enhance their interdiction capabilities and ultimately reduce the overall prevalence of these illicit substances. Current studies performed by the United Nations Office of Drug and Crime (UNODC) has indicated an increase in the amount of ATS seized within the last 4 years. The increase however has been accompanied by a decrease in the number of small scale laboratories found manufacturing these illicit substances [19-21]. The correlation, though counter intuitive, is as a result of illicit manufacturers switching production from numerous small scale facilities to less numerous large scale facilities meaning that there are now fewer facilities producing more illicit substances overall. The reduction in production facilities indicates a consolidation of sorts on the part of the illegal operators [21]. The consolidation simplifies the process of linking different batches of seized substances by law enforcement to a manageable database of illegal producers, meaning more confiscated ATS will be linked to each supplier. Ultimately linking different confiscated batches of drugs leads to larger criminal penalties being levied during trials. Researchers in Japan have already utilized other techniques to generate these data showing its significance to law enforcement [22-24]. One research group in Japan was 
able to reveal through other chiral separation techniques, the regional origin of seized ATS, by showing a trend in enantiomeric impurities in manufactured ATS from Asia separate from those manufactured in Europe and America [25]. The capabilities to link batches of ATS are only realized through highly specific chiral reactions, which have traditionally been out of the reach of the typical crime laboratory. However, through the use of Ion Mobility Spectrometry, a significant tool will be provided to law enforcement that will allow these types of analyses to be common place.

The ability to detect thermally labile substances in the form of explosives has been a long standing difficulty for law enforcement. Being able to detect ever smaller amounts of clandestine explosive compounds in pre-blast and post-blast samples is paramount in preventing the detonation of these devices and for information gathering exercises in post-blast situations [26]. Law enforcement are constantly seeking new and improved tools which may be used to detect such compounds, particularly in pre-blast scenarios to prevent a potential loss of life. Ion mobility has been able to achieve the level of detection without the compromise of lengthy analysis times or poor detection capabilities as seen with traditional chromatographic techniques such as GC and LC [27-34]. The heart of IMS is the ionization being used, in the case of the present study ESI which is a soft ionization technique will be used. Soft ionization allows for the thermally labile analyte to be ionized without analyte deterioration, allowing for improved transmission of analyte into the analytical system and overall improved detection capabilities. The use of soft ionization for the detection of thermally labile compounds is a very common practice in analytical chemistry and has been the analytical method of choice for the detection of these substances $[17 ; 28 ; 33 ; 35-40]$. However, numerous fiscal limitations faced by law 
enforcement agencies has restricted their use of specialized equipment often required to conduct these tasks, resulting in the reliance on general purpose instrumentation often not suited for the detection of these compounds. As reported by Flanigan et al., " the ability to detect a broad class of explosives ... with a single instrument is significant [41] ". Indeed, the dual functionality of the discussed ESI-IMS system would allow for well suited detection of thermally labile explosive compounds for law enforcement as well as the detection of the other specialized substances such as chiral impurities found in ATS. 


\section{OVERVIEW OF ION MOBILITY SPECTROMETRY}

Ion Mobility Spectrometry combines the techniques of atmospheric pressure chemical ionization (APCI) [42] and analyte separation by time of flight (TOF) under the influence of an applied electric field. There are several atmospheric ionization sources that have been utilized with IMS including photo-ionization (UV), laser ionization, electrospray,

corona discharge and radiation using americium $\left({ }^{241} \mathrm{Am}\right)$, tritium $\left({ }^{3} \mathrm{H}\right)$ or nickel $\left({ }^{63} \mathrm{Ni}\right)$ [1;6]. The most common of these is radiation using a 10 milli Currie ${ }^{63} \mathrm{Ni}$ source that emits beta particles with maximum energy of approximately $64 \mathrm{KeV}[1]$ and an average energy of $17 \mathrm{KeV}$ [7]. Other radioactive sources such as americium $\left({ }^{241} \mathrm{Am}\right)$ and tritium $\left({ }^{3} \mathrm{H}\right)$ provide ionization at different energy levels with $5.4 \mathrm{MeV}$ of alpha particles $[6 ; 43]$ and $18.6 \mathrm{KeV}$ of beta particle [43] respectively. Research has also been conducted in developing non-radioactive sources with similar ionization energies to radioactive sources, such as electrical discharge tubes developed by Gunzer et al. that emits beta particles with similar ionization energy to a tritium source [43]. In its most simplistic form, an IMS is comprised of two regions, the ionization or reaction region where samples are introduced and ionized, and the drift tube which is under an applied electric field that separates these ions (Figure 3). 


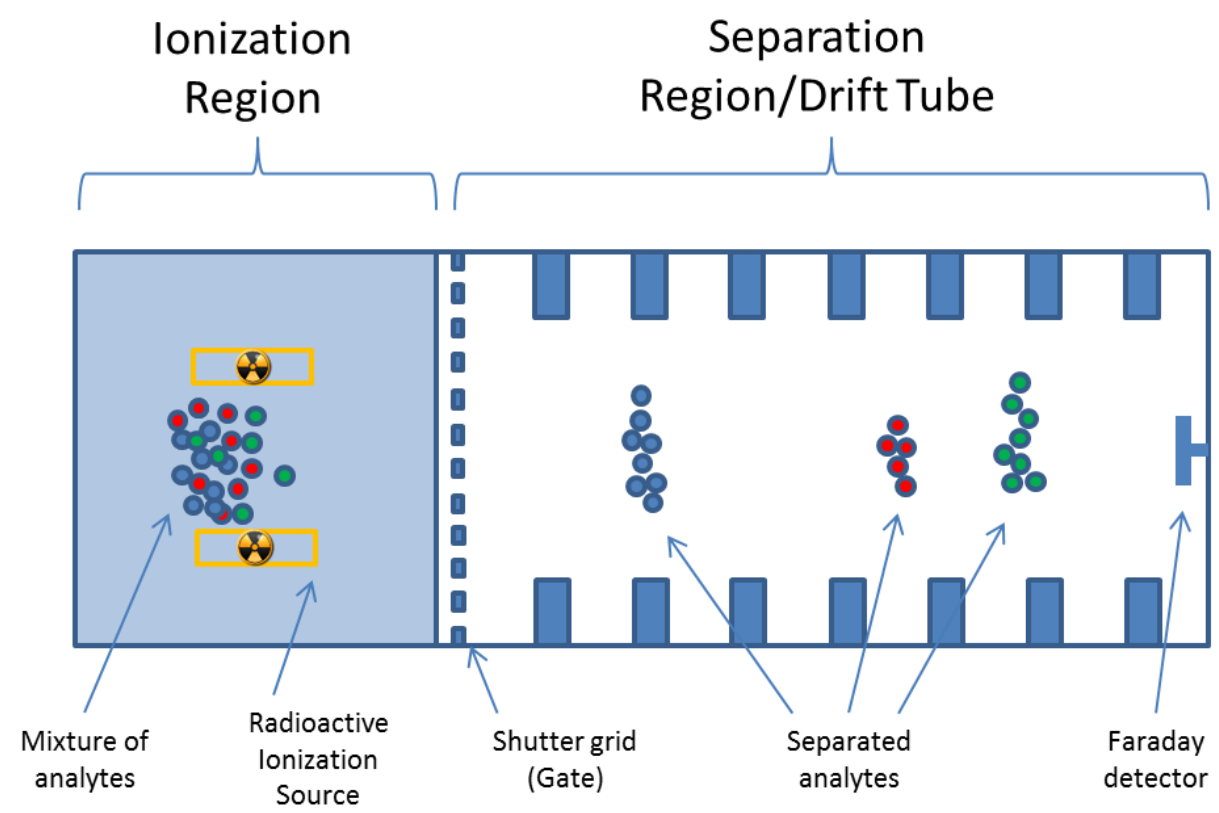

Figure 3: Ion Mobility Spectrometer

Many commercial IMS units are capable of analyzing samples in either a solid or liquid form. Samples are collected on an absorbent material, which is then heated by a hot desorption port to vaporize the sample into the ionization region of the IMS. Once in the ionization region, competitive ionization occurs as the ${ }^{63} \mathrm{Ni}$ ionizes the surrounding atmospheric vapor (drift gas) which is usually mixed, referred to as being "doped", with a reactant gas. The reactant gas becomes ionized and will then transfer charge to the vaporized analyte. The analyte which is now ionized will be "injected" or pulsed into the drift tube via an electric gate or shutter grid within the drift tube at an applied electric field of 200 to $400 \mathrm{~V} / \mathrm{cm}$ [8]. The electric field causes the ions to migrate at a particular rate (mobility) in the presence of an opposing drift gas. A combination of these two forces, drift gas and electric field, will cause ions of different mass to charge and shape to charge ratios to separate [1]. The collision of these ions with a faraday cup at the end of 
the drift tube will result in an increase in current of 10 to $1000 \mathrm{pA}$ which is amplified and interpreted by data processing software [1]. The resulting peaks elute in milli-seconds and a plot of intensity vs. time is generated and called a plasmagram (Figure 4).

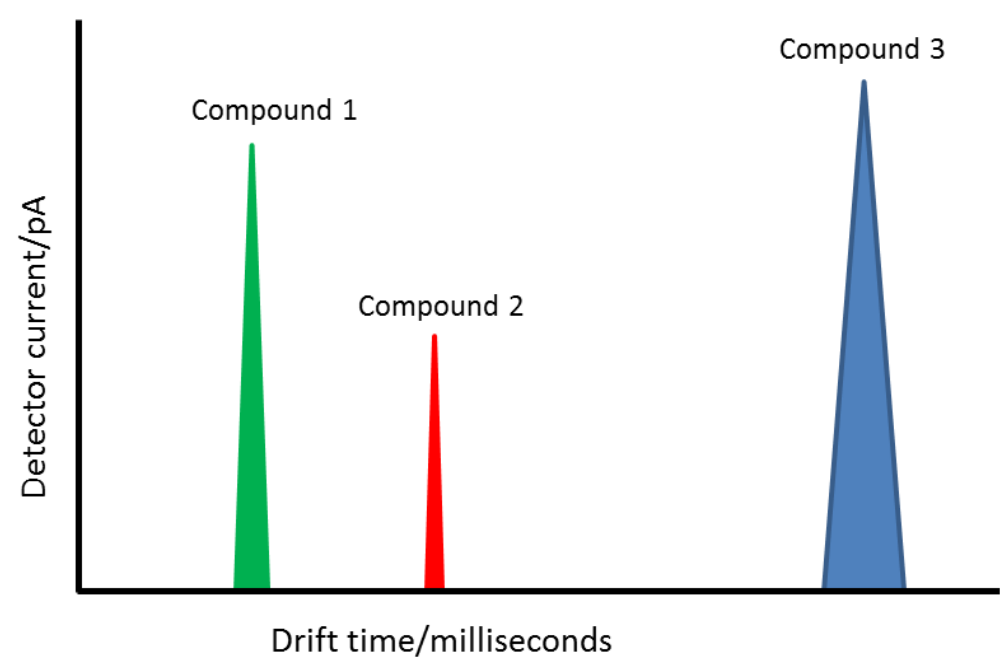

Figure 4: Typical IMS Plasmagram

The rapid analysis time of IMS for the separation and detection of analytes is one of its greatest strengths and is exploited in various industrial applications such as the evaluation of waste sites for toxic chemicals, purity of polymers, space exploration, food, industrial pollutants and forensics [6]. The high versatility of IMS, satisfactory analyte separation capabilities and the relatively low design and acquisition costs have also given it the name "poor man's mass spectrometer"[6]. Other drift tube designs such as travelling wave IMS and field asymmetric IMS have also seen more frequent use in recent years [44]. Each of these drift tubes provides enhanced separation capabilities for specific applications, however only the linear drift tube design as shown in Figure 3 was used in the course of the work and so discussion of other IMS drift tubes will be limited. 


\subsection{Theory of Electrospray Ionization}

The process of Electrospray ionization (ESI) is not limited to laboratory-based analytical techniques. Electrospray ionization is used in a wide array of industrial applications from electrostatic painting in the automobile industry to crop dusting within the agricultural industry [45]. Electrospray ionization has the ability to produce a stream of fine droplets in air that contain ionized particles through the application of an electrical charge to a solution. The process works more effectively with solutions that are conductive and with analytes that are either Lewis acids or Lewis bases. The exact mechanism of how charge is transferred from the electrospray electrodes to the solution and then from solution to neutral analytes is not completely understood. However, this particular process may be explained utilizing redox equations and considering electrospray ionization as a voltaic cell (Figure 5) [46;47].

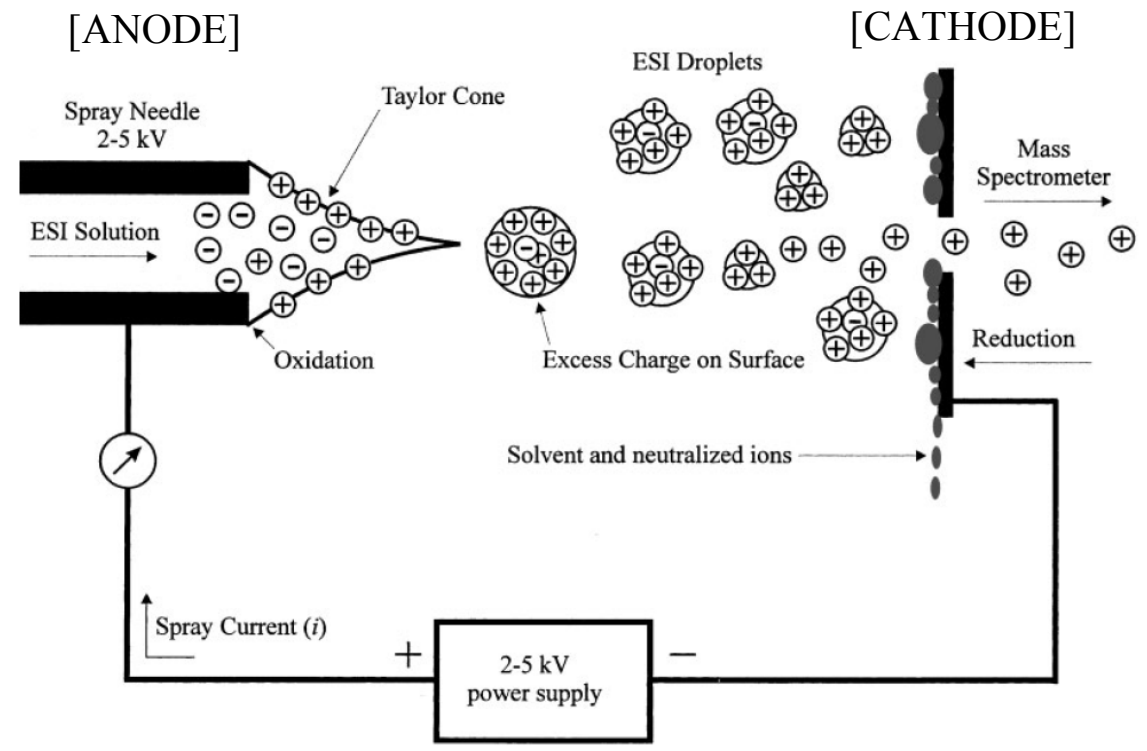

Figure 5: Illustration of ESI as a chemical Redox reaction cell [46] 
The redox equilibrium allows a low energy transfer of charge from an anode in the positive ion mode, through the solution to produce ions. The ions will migrate in the gas phase towards the cathode to complete the circuit, thereby creating a spray of ions. The process may be considered as an electrolytic cell, where oxidative and reductive processes occur under the influence of an applied potential may be expressed using Equation 3 [48].

Equation 3: Probable redox reactions occurring during electrospray ionization in positive ion mode at the anode (ESI needle).

$$
\begin{gathered}
2 \mathrm{H}_{2} \mathrm{O} \rightarrow 2 \mathrm{H}^{+}+\mathrm{O}_{2}+2 e^{-} \\
\mathrm{H}^{+}{ }_{(a q)}+\mathrm{H}_{2} \mathrm{O}_{(l)} \rightarrow \mathrm{H}_{3} \mathrm{O}_{(a q)}^{+} \\
\mathrm{H}_{3} \mathrm{O}_{(a q)}^{+}+A_{(a q)} \rightarrow A H_{(a q)}^{+}+H_{2} O_{(l)}
\end{gathered}
$$

The first line of Equation 3 shows the oxidation of water from the aqueous ESI solution which results in a loss of electrons at the anode (ESI needle), these electrons move as a result of the potential difference between the ESI needle surrounding the solution and the counter electrode (cathode) displayed in Figure 5. The flow of electrons or electrical current from the oxidative process produces free protons within the solution that further oxidize the dissolved neutral analyte (A) within the electrospray solution, producing protonated species $\left(\mathrm{AH}^{+}\right)$(Equation 3). These protonated species are further repelled by the high potential of the ESI electrode unto the counter electrode creating the stream of fine droplets that contains these gas phase ions. A fairly large amount of charge accumulates within these droplets causing similarly charged particles to repel each other. The repulsion or columbic stress causes an "explosion" of charged solvent resulting in 
droplets being dispersed into progressively smaller sizes (Figure 6). As a result of this dispersion, the ESI process effectively ionizes and de-solvates the analyte producing "naked" gas phase ions from either volatile or non-volatile analytes.

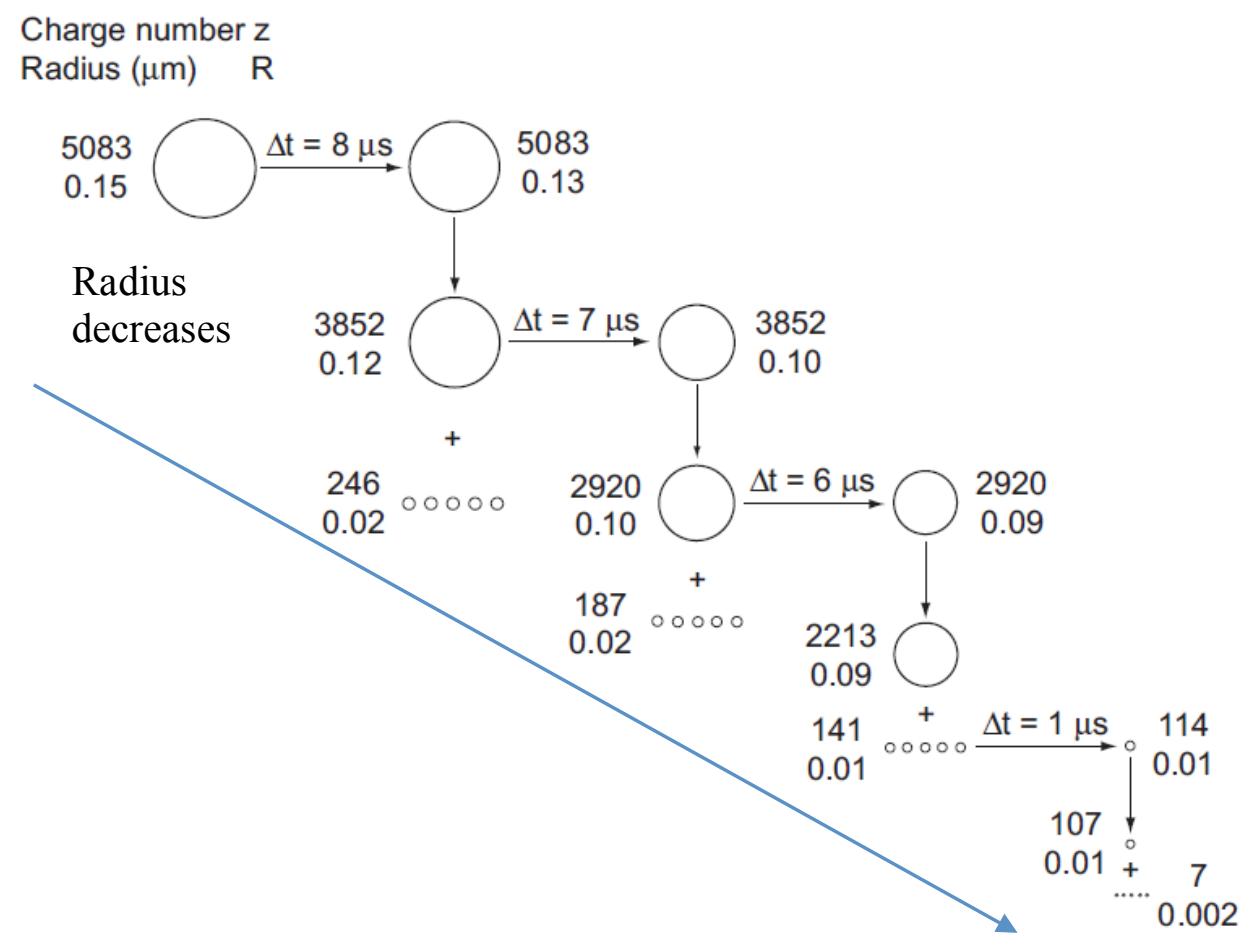

Figure 6: Dispersion of a droplet formed under nanoESIspray conditions [47]

The fact that ESI can create ions from both volatile and nonvolatile analytes within solutions has allowed ESI to become ubiquitous for mass spectrometric applications involving liquid separation techniques. Electrospray ionization has quickly surpassing other similarly developed liquid-based ionization techniques such as fast atom bombardment (FAB), thermo-spray and the monodisperse aerosol generating interface for chromatography (MAGIC). Each of these techniques has failed to match the simplicity that accompanied utilizing ESI and as a result are rarely used during routine analyses 
today [45]. The electrospray process itself is simplified, an aerosol spray of fine drops is created as a result of a high electric potential being applied to a needle containing a solution with a polar solvent [45] which is referred to as a field desorption technique [49]. The electrospray ionization process allows for the creation of charged ions of intact molecules as a result of its inherently low ionization energy of $\sim 10 \mathrm{eV}$ [50] from ESI. Low ionization energy has made ESI popular for the ionization of large biomolecules that are typically too fragile to be ionized by any other means. Electrospray ionization has allowed the analysis of relatively large and labile biomolecules through techniques such as mass spectrometry. The adaptation of ESI to IMS has been a natural progression over time as there have been numerous other ionization sources coupled to IMS devices as previously mentioned. Electrospray ionization still allows for the analysis of large biomolecules through soft ionization but also has the added advantage of not containing a radioactive source typically found in many off the shelf (OTS) IMS devices. Using a nonradioactive source such as ESI has reduced what has been referred to as the "bureaucratic burden" that accompanies housing traditional radioactive sources. The burden requires that the holder of these radioactive sources comply with Environmental Health and Safety regulations to minimize potentially lethal exposure and/or loss of these sources. The health and safety requirements have limited the use of many OTS IMS devices and may be attributing to IMS' anemic growth within academia. Credit for demonstrating Electrospray Ionization goes to Malcolm Dole who in 1968 reported that it was possible to obtain intact gas-phase macromolecules under atmospheric conditions [51]. As Dole stated in his Molecular Beams of Macroions paper, "the dogma at that time was that drops on evaporation would become electrically unstable and break down into smaller 
drops until only one macromolecule per drop would result". Dole continues by stating that the macroions formed would be vibrationally unexcited and so would not fragment [51]. Put simply, the ESI process was of low enough energy to ionize large molecules without causing destruction or fragmentation. The end result being a charged macromolecule that would be devoid of solvent and still be intact after ionization due to the low energy level transfer of charge (Figure 6).

\subsection{Mechanics of the Electrospray Process: The Taylor Cone}

The heart of electrospray ionization is the formation of fine droplets from a liquid under the influence of an applied electric field. The fine droplets that form result from a distortion of the liquid at the tip of the nozzle/capillary into a fine point commonly referred to as the "Taylor cone" named after Sir Geoffrey Taylor [52]. Sir Taylor was investigating the distortion of water droplets in strong electric fields which are common during thunderstorms. Through several experiments, Sir Taylor reported that under the influence of an electric field a drop of liquid will become elongated until its length becomes 9 times its equatorial diameter. At that point, the drop becomes unstable as a consequence of the influence of the electrostatic field and the surface tension of the liquid, resulting in an elongated sphere (Equation 4). By increasing the electrical potential that results in greater electric field strength, Lord Rayleigh reported that jets develop out of the unstable ends of the drops. "The liquid is thrown out as fine jets, whose fineness, however, has a limit" [53].

Equation 4: Relationship of voltage and shape of a drop that determines the ESI Taylor cone.

$$
V=\left(4 \pi(n+2) r_{0} T\right)^{\frac{1}{2}}[52]
$$


The limit of the ESI droplet was reported by Taylor as occurring when the obtuse end of the drop elongates to a point that measures an angle of approximately $49.3^{\circ}$ [52]. As shown in the figures below, under no electric field (0V) Figure 7 and strong electric field (3.5KV) Figure 8, the drop has become elongated and has now formed an angle of approximately 98.6 as indicated by the red lines overlaid on Figure 8 .

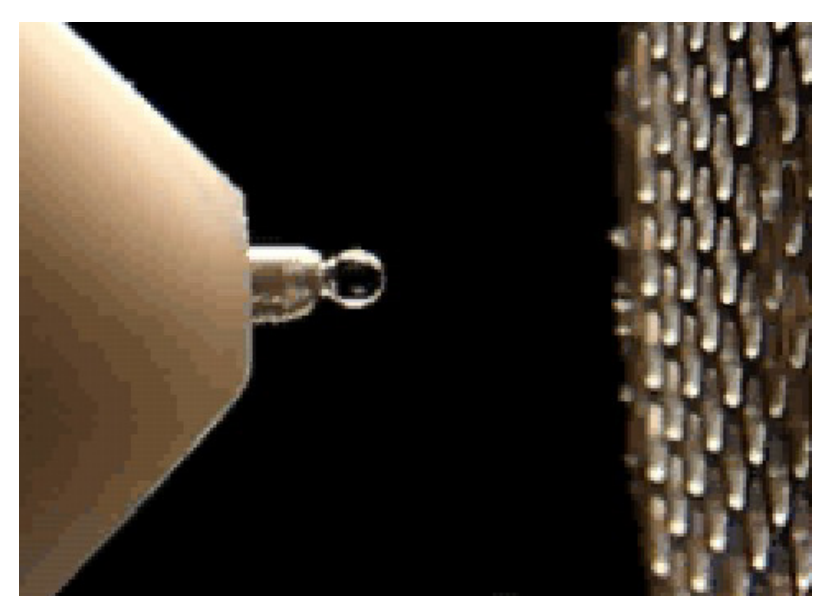

Figure 7: Spherical drop formed at ESI tip under no electric field (0KV)

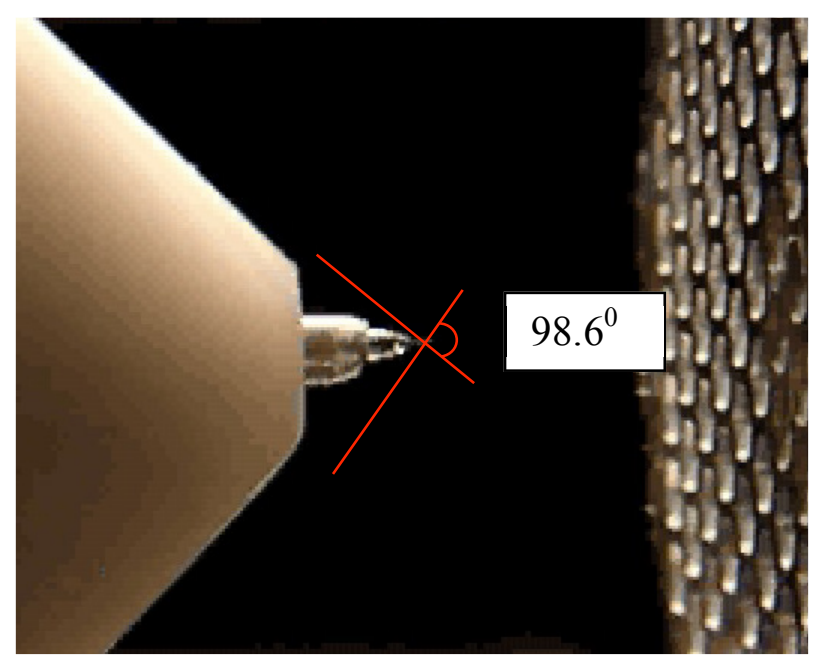

Figure 8: Elongated drop formed at ESI Tip under strong electric field (3.5KV) 
The fine spray that results from the end of the tip is responsible for the formation of gaseous ions. The cone itself at the tip is referred to as the "Taylor cone" that occurs under all true electrospray conditions. True electrospray conditions refer to the generation of these gaseous ions in the absence of corona discharge or pneumatic nebulization. True ESI is not limited to only occur without these phenomena, but it has been reported that corona discharge which occurs under excessive electric field strength is a different ion generation process that inhibits ESI. Conversely, pneumatic nebulization which is intentionally done to assist ESI when using higher liquid flow rates, reduces its overall efficiency [49]. The ability of ESI to create gaseous ions from non-volatile species is one of its strongest attributes. Electrospray ionization is able to perform this transition through field desorption activation. Desorption methods utilize strong electrostatic fields to extract ions from a substrate. Field desorption was pioneered by Beckey in the 1960's and utilizes a substrate with sharp points called whiskers or dendrites [54]. The sharp points assist in accumulating charge due to their inherently small radii (Figure 9). The analyte is then placed on the substrate where under a strong applied electric field, an electron is removed from the analyte forming a charge radical $\left(\mathrm{M}^{+}\right)$. The ionization itself is very soft, even more so than that observed from energy sudden methods such as laser desorption (LD), fast atom bombardment (FAB) and secondary ion mass spectrometry (SIMS). Field Desorption closely resembles ESI, as both create ions from analytes in a liquid medium. Both transfer very little energy to the analyte, but the original field desorption (FD) developed by Beckey creates intact molecular ion radicals. Field desorption ionization was one of the first soft ionization techniques utilized that could be used for the analysis of volatile analytes by allowing the gaseous analyte to be blown 
over the substrate. Or, it could be used to ionize non-volatile liquids, where the analyte was either deposited directly on the substrate, or a small continuous flow was allowed onto the substrate. Field desorption resulted in simple to interpret spectra from nonvolatile aqueous samples which was previously unobtainable. However, the preparation of these substrates or electrodes and continuously dosing them with analytes for analysis proved difficult. As a result, this technique though useful fell out of favor among researchers and was soon abandoned, leaving behind electrospray ionization ESI as the most practical and now as the most popularly used atmospheric ionization technique for liquids.

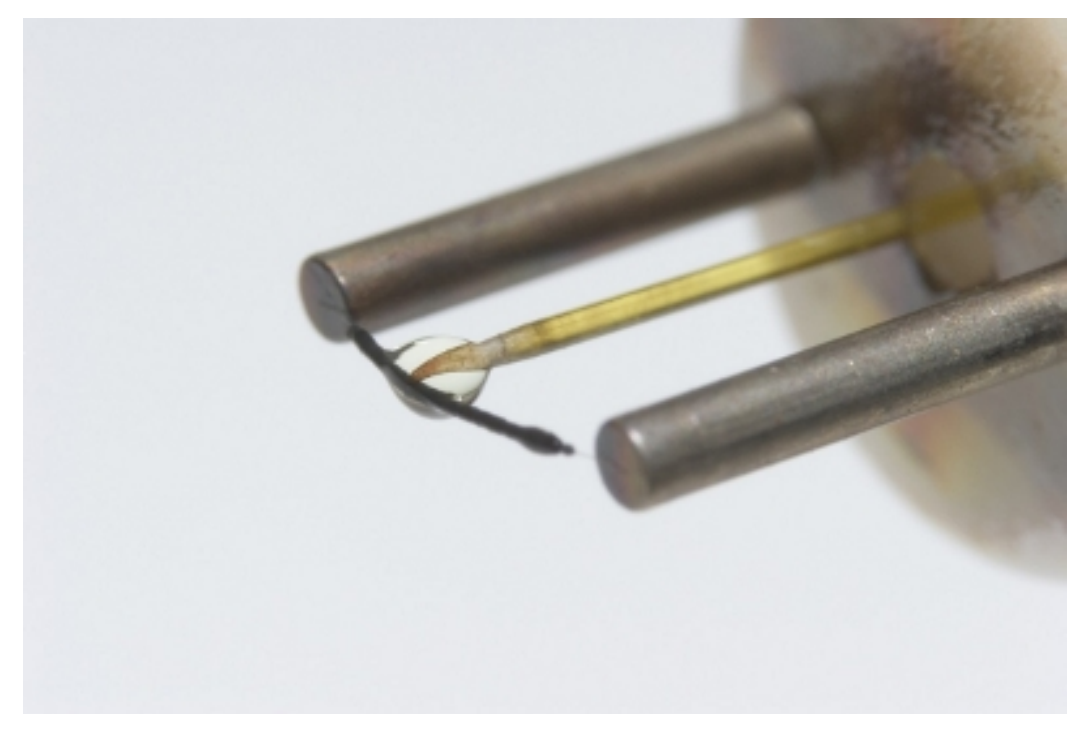

Figure 9: Image showing continuous flow of analyte onto field desorption sorbent http://www.udel.edu/chem/ms/lifdi.html

\subsection{History of Mass Spectrometry}

Mass spectrometry is the study of charged atoms or molecules defined by the ability to separate these analytes by their mass (m) to charge (z) ratios. The capability to separate 
ions based on mass to charge was first demonstrated in 1918 by Jeffery Dempster [55] and later by Francis William Aston in 1919 [56;57]. The applications of mass spectrometry have revolutionized the science of gas chromatography whereby compounds are now almost exclusively detected by mass spectrometry. The application of mass spectrometry for the detection of larger non-volatile molecules soon followed with liquid chromatography applications. However the hardware required to make a complete and functional system is cumbersome, expensive and difficult to maintain. The problem has and still is efficient ionization of the liquid or solid sample for separation and analysis by the mass spectrometer. As a result, much of the emphasis for studying these large molecules by mass spectrometry has focused on development of the ionization used to introduce the sample rather than the mass spectrometer itself used to analyze the sample. Various techniques have been developed that ionize these non-volatile matrices. These include electrospray ionization (ESI) previously discussed as the most popular, atmospheric pressure chemical ionization (APCI), matrix assisted laser desorption (MALDI), desorption electrospray ionization (DESI) and direct analysis in real time (DART). All these interfaces/ionization sources allow the introduction of intact ions from solid or liquid matrices into a mass spectrometer for analysis.

All mass spectrometers, have three essential components; an Ionization Source, Mass Analyzer or Mass Selector and a Detector. Crucial to the operation of any mass spectrometer is also the vacuum system, since ionic species are highly reactive, they must exist in a relatively inert atmosphere so that they can attain a high "mean free path." The mean free path is the average distance travelled by a molecule before it has a collision with another molecule. The mean free path will ensure that ions created for analysis, will 
not be destroyed by unwanted reactions before they get to the detector. The mean free path is achieved using a vacuum system that will remove any unwanted molecules from interacting with the ions created. The high vacuum requirement is the main obstacle in coupling mass spectrometers to any other non-vacuum dependent analytical system such as ion mobility. Since ion mobility systems generally operate at comparatively high pressures and gas flow rates, the transition of analytes and solvent from high pressure to vacuum results in an expansion which overwhelms the mass spectrometer's vacuum system. High pressure and gas flow creates far too many molecules within the mass spectrometer, reducing the mean free path of the ions of interest and reduces the overall detection capabilities of the instrument. Today, good mean free path has largely been accomplished through the utilization of high capacity vacuum pumps and sample skimmers to quickly reduce the effects of sample introduction into the mass spectrometer. In order to analyze very high molecular weight molecules, the capability of the mass spectrometer to detect these large masses limits the type of mass analyzers used for analyses. Today the two mass analyzers with extremely wide mass sampling ranges are ion cyclotron and time of flight. Both these mass analyzers have a near unlimited mass range but their complexity increases their acquisition cost eliminating their use for the project. Instead, a quadrupole mass analyzer was found sufficient to provide all the separation capabilities required.

\subsubsection{Mass Analyzers}

There are several types of Mass Analyzers commercially available, these include but are not limited to: Quadrupole which is the most common used, Ion Trap, Time of Flight, Magnetic Sector and Ion Cyclotron [58]. Each Mass Analyzer or Mass Selector 
mentioned has its own strength and weakness. Both quadrupole and ion trap mass analyzers are static analyzers that have limitations in the mass ranges they can analyze, generally 50 to $2000 \mathrm{amu}$ [59]. These analyzers filter out or trap ions of a particular mass to charge ratio through the utilization of alternating electric fields. The low mass range limits the utilization of these mass analyzers for mass ranges in excess of 2000 atomic mass units. However, a time of flight (TOF) mass analyzer equipped with an ion reflector has an almost unlimited mass range as these ions are separated on the basis of the time traveled through the field-free region [60]. The unlimited mass range coupled with the high sampling rate of the TOF electronics makes it perfectly suited for the detection of ions produced intermittently by pulsing a packet of ions such as within an Ion mobility spectrometer. Ion cyclotron and magnetic sector mass analyzers are also well suited for work with large molecules, but the costs of these particular instruments prohibit their widespread use. The ubiquitous quadrupole mass analyzer was selected for this project as a result of the relatively low acquisition cost as well as the widespread expertise already developed in utilizing these mass filters.

\subsubsection{Coupling IMS to Mass Spectrometry}

One of the earliest reports of coupling IMS to a Mass Spectrometer was done by McDaniel et al., who utilized a drift tube operating under low electric field and low pressure to study the reactions of various gases such as hydrogen in 1962 [61]. During that study, the drift tube was utilized to vary the number of collisions of the ion molecules prior to being analyzed by the mass spectrometer. The study was undertaken in an attempt to determine the ionic species obtained under varying drift conditions through mass spectrometric confirmation. In essence, the mass spectrometer was being used to 
enhance the data obtained from conducting ion mobility experiments. Today, ion mobility spectrometry has become so mature that it is now being used as a tool to enhance mass spectrometric analysis. The enhancement of other techniques by IMS is particularly true in biochemical analysis where analyte ions are often structural isomers and hence isomeric $[44 ; 62 ; 63]$ and unable to be resolved through the use of mass spectrometry. Ion mobility spectrometry has also been used to enhance mass spectral analysis by pre-screening analytes prior to their entry into the mass spectrometer, allowing for cleaner spectra and overall greater sensitivity by reducing the level of noise through elimination of unwanted analytes from entering the detector (Figure 10).

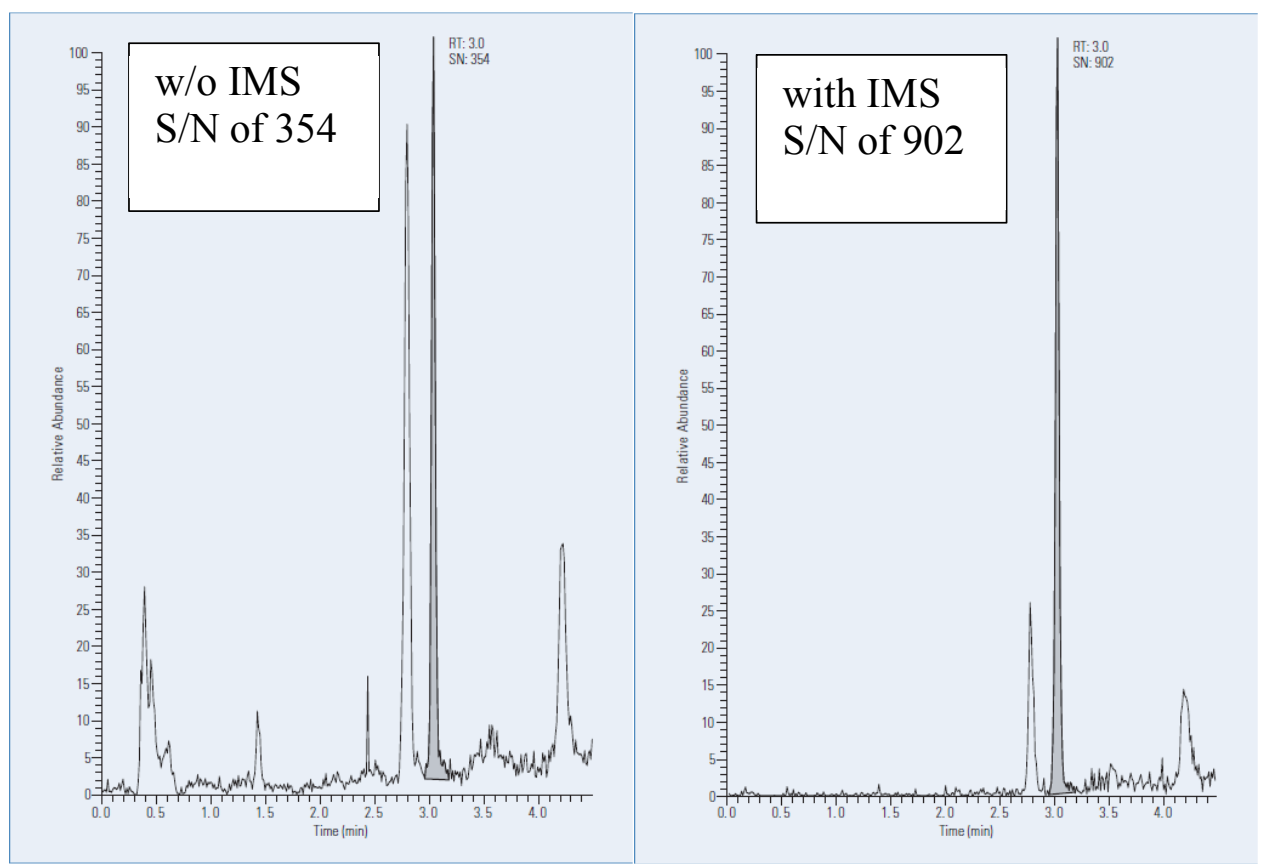

Figure 10: Improvement in signal to noise (S/N) obtained by using IMS coupled to MS [64]

Such improvements have been beneficial for both techniques (IMS and MS) as IMS on its own is unable to provide unambiguous confirmation of the existence of a particular analyte, while mass spectrometry requires additional resolving capabilities to readily distinguish isomeric species. The coupling of the two has been synergistic, as each has 
provided additional capabilities by giving information that neither could produce on its own, making the resulting union greater than the sum of each individual technique. However, the coupling of these two techniques does present analytical challenges. The first challenge is that both ion mobility and mass spectrometry are destructive techniques. That is, in order for either technique to detect an analyte ion, it must effectively neutralize that ion making it non-existent. Coupling either of these techniques therefore creates a challenge as to how ions will be transported from one detection system to the other. The solution currently used by ion mobility spectrometrists is to eliminate a portion of the IMS' faraday detector so that not all ions are detected and allow some of the remaining ions to be fed into the mass spectrometer [65]. By eliminating a portion of the faraday detector, there is some reduction in sensitivity on the part of the IMS, but the overall enrichment in data generated by the mass spectrometer compensates for this slight decrease in sensitivity. The second challenge has been far more elusive to resolve, as it rests in the very nature of either technique. Ion mobility spectrometry is lauded for its high speed separation capabilities, resolving mixtures on the order of milliseconds, however, mass spectrometers require far more time in order to provide a resolved mass spectrum of an analyte, on the order of microseconds to several minutes [65]. The thousand fold difference in analysis times creates a significant hurdle in introducing, analyzing and recording data for IMS-MS coupled systems. Resolutions for the challenge in acquiring IMS-MS data have ranged from performing repeated and continuous IMS analysis to provide sufficient ion time for typically slower mass spectrometers to collect data, or utilizing dual gated IMS systems that will inject only a single packet of an ion swarm for analysis by the mass spectrometer. The reason for the wide range in scan times 
of mass spectrometers stems from their mass analyzers. Each mass analyzer separates ions by different mechanisms. Magnetic sector analyzers separate ions on the basis of changes in momentum within a magnetic field. To allow these changes, a continuous stream of ions is required necessitating a longer residence time than that allowed from IMS analysis. Quadrupole instruments separate ions on the basis of their stability within an electrodynamic field and so suffers from similar time constraints as magnetic sector analyzers. Hyperbolic ion traps are able to store ions however, the precise timing involved to store sufficient ions from the short analysis times of IMS analyzers is still problematic. However, Time of Flight (TOF) analyzers separate ions in similar principles to drift tube ion mobility spectrometers and are generally equipped with high speed electronics to capture the rapid separations that occur during flight in the microsecond scale. By comparison, IMS analyses are relatively slow to the detection speed of TOF mass analyzers, making TOF analyzers an ideal and popular choice when coupling IMS to MS [65]. The third challenge encountered when coupling IMS to MS has been the same challenge when coupling any technique to MS. The fact that MS must operate under high vacuum (milliTorr pressures), significantly reduces the types of interfaces that may be used, compounding this is the fact that IMS operates at ambient to high pressure with high flow rates of drift gas flowing through the drift tube. The high pressure and flow rates have required MS devices with significant pumping capabilities in order to maintain the low pressures necessary to facilitate MS analysis when these are coupled to IMS systems. A detailed report was created by the Sandia National Lab describing the pros and cons of coupling various mass spectrometers to IMS devices as well as the steps 
required in optimizing the interface between both detection systems [65]. Many of these steps will be explained in detail within the remaining chapters.

\subsection{Performance of Electrospray Ionization versus Nickel-63 Ionization.}

Ion mobility spectrometry (IMS) is a stand-alone separation technique that has been categorized similarly with gas chromatography and liquid chromatography [66]. The term ion mobility describes the motion "mobility" of ions under the influence of an applied electric field. The ion swarm as it is referred to in IMS will drift/migrate at a rate proportional to the intensity of the applied electric field. Ions with relatively small masses and high charge (low mass to charge ratio) will migrate faster and have higher mobility than ions with relatively large mass and low charge (high mass to charge ratio). The difference in mobility between low and high mass to charge ions results in the desired separations. The ion swarm also interacts with the surrounding neutral atmosphere which in turn causes separations based on the ions shape to charge, as ions with relatively large collisional cross sectional areas will have slower migration rates or mobilities than those with smaller cross sectional areas [1]. These basic separation mechanisms are true for all "drift tube" or "linear tube" IMS units [44]. What has altered IMS is the proliferation of front end ionization sources that creates this initial ion swarm. As previously mentioned, radioactive sources made of isotope Nickel-63 is commonly used in commercially available off the shelf (OTS) IMS units. Other ionization sources used include Desorption Electrospray Ionization (DESI), Direct Analysis in Real Time (DART), Matrix Assisted Laser Desorption Ionization (MALDI), Corona or Glow Discharge and last but not least Electrospray Ionization (ESI) [6]. Each ionization source has its strengths in particular 
applications, but almost all suffer from interferences during ionization that requires an intermediate buffer or doping gas to minimize these interferences. Electrospray ionization however, does not suffer from such interferences as electrospray ionizes analytes that are directly dissolved within the spraying solution. As a result, ionization with ESI becomes more direct and more specific based on the analytes dissolved and hence the spectra obtained from ESI not only reveals intact ions due to the soft ionization, but are also cleaner and simpler to interpret. In order to maintain the high sensitivity currently observed in radioactive $63 \mathrm{Ni}$ source based IMS, a high ion flux or high generation and transmission of ions from the ionization source through the IMS drift tube to the detector is required. Radioactive sources which are the de-facto means of ionization for many IMS units have very high ionization potentials allowing them to ionize a large number of neutral molecules. Nickel-63 for example has a maximum ionization energy of $67 \mathrm{KeV}$, for an electron (beta particle) emitted from the source. The electron will lose approximately $35 \mathrm{eV}$ of energy to ionize a neutral analyte, leaving it with sufficient energy to further analyze other neutral species producing up to 2000 ion pairs per beta particle [67] (see equation below).

$$
\begin{aligned}
& \text { Equation 5: Ionization of air molecules by nickel beta particles } \\
& \qquad \begin{array}{c}
\mathrm{N}_{2}+\beta^{-} \rightarrow \mathrm{N}_{2}^{+}+\beta^{\prime}+e^{-} \\
\mathrm{N}_{2}^{+}+2 \mathrm{~N}_{2} \rightarrow \mathrm{N}_{4}^{+}+\mathrm{N}_{2} \\
\mathrm{~N}_{4}^{+}+\mathrm{H}_{2} \mathrm{O} \rightarrow 2 \mathrm{~N}_{2}+\mathrm{H}_{2} \mathrm{O}^{+} \\
\mathrm{H}_{2} \mathrm{O}^{+}+\mathrm{H}_{2} \mathrm{O} \rightarrow \mathrm{H}_{3} \mathrm{O}^{+}+\mathrm{OH} \\
\mathrm{H}_{3} \mathrm{O}^{+}+\mathrm{H}_{2} \mathrm{O}+\mathrm{N}_{2} \rightarrow \mathrm{H}^{+}\left(\mathrm{H}_{2} \mathrm{O}\right)_{2}+\mathrm{N}_{2}
\end{array}
\end{aligned}
$$


By comparison, electrospray ionization is believed to have a much lower ionization potential of between $11 \mathrm{eV}[50]$ and $17 \mathrm{eV}[1]$ versus the $67 \mathrm{KeV}$ for ${ }^{63} \mathrm{Ni}[1]$. However, for ESI there is believed to be a direct interaction between the analyte and the source (Equation 3) as opposed to an indirect relationship that exists with radioactive sources (Equation 5). The indirect interaction for radioactive sources significantly reduces their effective ionization potential of many gas phase ionization sources. Electrospray ionization, though having lower ionization energy is able to apply more of that energy directly to the analytes of interest allowing it to still create a large ion flux despite a lower ionization potential. Several studies have revealed that by limiting the flow of sample to ESI sources, one is able to achieve even greater ion flux resulting in higher detection capabilities [68]. Though seemingly counter intuitive, ESI responds in an inversely proportional manner when relating flow rate to the ion current generated [45]. As a result, careful consideration of the flow rate of the effluent being introduced to an ESI source will greatly affect the ion current generated and ultimately the detection capabilities of a particular instrument. In 2003, one of the founding fathers and revolutionaries of IMS, Dr. Gary Eiceman, was quoted as saying "We're all looking for a small, low-power ion source that replicates ${ }^{63} \mathrm{Ni}$ with comparable gas-phase chemistry, without radioactivity" [8]. Electrospray ionization has been proven to be an alternative ion source to traditional radioactive ionization sources with current published reports on IMS utilizing either ESI or ${ }^{63} \mathrm{Ni}$ as the ionization source. Most samples received within a laboratory setting require some type of sample pretreatment which generally entails dissolution in a suitable solvent that allows easier handling and manipulation. As a result, samples currently used within crime labs are readily amenable to analysis via electrospray ionization. The added 
advantage of electrospray ionization is the ability to analyze nonvolatile analytes which has been difficult when utilizing traditionally ionization sources such as ${ }^{63} \mathrm{Ni}$ as these are more suited for gaseous samples which are traditionally not the most common state of matter for evidence submitted to crime laboratories.

The first phase of ion mobility analysis, is the ionization of a sample from a neutral molecule to an ion, whether positive or negatively charged. Though there have been numerous attempts to couple various ionization sources to IMS in the past [69], none have required significant changes to the second phase of IMS which is the separation section or drift tube. As a result, regardless of the ionization source coupled to IMS, the separation obtained in theory will be unaffected. However, electrospray ionization has been reported to provide peaks that are slightly narrower and hence have a higher resolving power than those obtained from other ionization sources [70]. The narrower peaks have been attributed to the additional ability of electrospray ionization to produce "naked" ions. These "naked ions" are simply gas phase ions that are essentially desolvated and have little to no additional adducted species. The creation of naked ions is key to having a tightly packed ion swarm. The tight swarm migrate down the drift tube resulting in sharp peaks with higher resolving power than that obtained when using traditional ionization sources such as photoionization or radiation using ${ }^{63} \mathrm{Ni}$ or ${ }^{241} \mathrm{Am}$ as previously mentioned. Since the first presentation of electrospray type techniques coupled with IMS during the 1972 Annual Conference on Mass Spectrometry and Applied Topics [71], numerous authors have gradually improved upon those initial experiments to enhance the overall application. The main champion of these developments has without a doubt been Dr. Herbert Hill, whose group presented one of 
the first reports of effectively coupling electrospray to IMS, initially coining the term "Corona spray-nebulization" in 1989 [72] and then finally refining the technique into what is currently the standard for ESI-IMS interfaces in 1994 [73]. As electrospray requires the analyte to be dissolved within an electrically conductive solvent, mixtures of polar solvents compatible to the analyte are used. These are typically aqueous/organic mixture also commonly used in liquid chromatographic systems (LC). During electrospray ionization, these solvents as well as the analytes are ionized, resulting in both naked ions and solvent ions being generated. However, since the separation which occurs within IMS does so under atmospheric conditions, the elimination of unwanted ions or neutrals that may steal charge during the separation process is crucial. Therefore, ionized solvent molecules, though not directly affecting the ionized analyte, will affect the separation characteristics and ion lifetimes within the drift tube. Minimization of these solvent ion effects in ESI-IMS has been achieved through the use of counter flow desolvation gases which have been placed at various locations within the ionization region of ESI-IMS units at varying temperatures. The major drawback being the ability of these gases to prematurely evaporate the injected analyte/solvent mixture before ionization can occur [73]. Thus, a cooled ESI source has been developed that allows the ionization of analytes under true electrospray conditions and also minimizes the introduction of solvent ions and neutrals that will affect the atmospheric separation of created ions once in the drift tube [74]. Additional steps to obtain superior ionization efficiency for ESI comparable to that obtained from ${ }^{63} \mathrm{Ni}$ have been in the design of the ESI needle itself. Micro-machining techniques (Figure 11) have advanced at such a pace 
that precisely cut conical fused silica tips are possible with openings as small as 1 micrometer to all for the most optimal electrospray conditions [68].
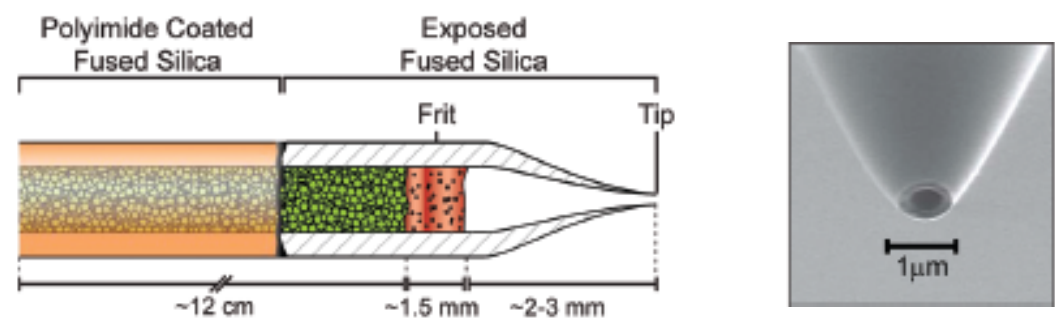

Figure 11: Micromachined Fused Silica tips used in ESI sources [68].

As previously mentioned, ESI displays a counter intuitive characteristic where higher ion flux is obtained at relatively low sample flow rates [68;70] (Figure 12). The rational being that low flow rates allow for true ESI conditions which are more efficient in generating ions than that obtained at high flow rate conditions. Flow rates in the order of microliters per minute [75] to as low as a few nanoliters per minute are typically used for ESI-IMS applications allowing for maximum ionization efficiency [68;76]. The formation of a stable Taylor cone under all ESI operations is what allows the technique to generate ions at the highest level of efficiency, the use of relatively low flow rates without the need to artificially nebulize the sample allows this phenomenon.

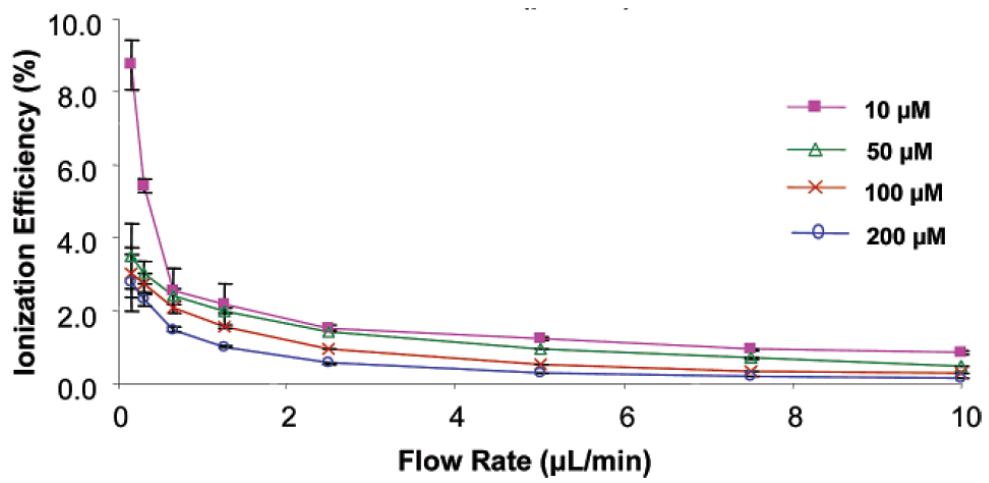

Figure 12: Plot of Ionization efficiency versus ESI flow rate [77]. 
The use of low flow rates is also in line with the dogma that ESI is a concentration rather than a mass based technique as stated by Griffiths et al. [78]. Alluding to the fact that ESI favors high concentration and low mass (low volume flow rates) in order to achieve maximum ionization efficiency and hence analyte sensitivity. However, the ideal ESI parameters do not simply begin and end by the selection of low flow rates. Extremely low flow rates as described for some ESI processes $(<1 \mu \mathrm{L} / \mathrm{min})$, though having high ionization efficiency would simply produce too few ions for sufficient detection of any analyte. Therefore, though low to ultralow ESI flow rates have greater ionization efficiency as shown in Figure 12, relatively high flow rates are still required in order to produce sufficient ions for IMS detectors to produce good responses to analytes (Figure 13). The major disadvantage being that while large amounts of ions are being produced at higher flow rates, there are equally large amounts of unwanted neutrals and solvent species also being loaded into the analytical system because of the lower ionization efficiency under these conditions.

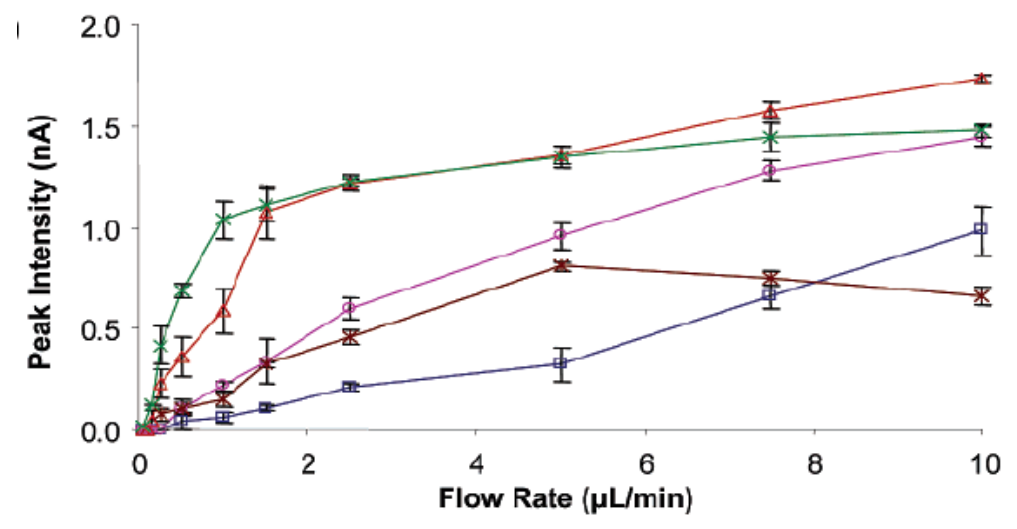

Figure 13: Plot of Ion current with ESI flow rate [77]

The introduction of relatively large amounts of water and other solvents into the IMS drift tube, has been the largest downfall with the utilization of electrospray ionization for 
IMS (Figure 14) [74]. To overcome this obstacle, various parameters have to be considered when utilizing ESI for IMS.

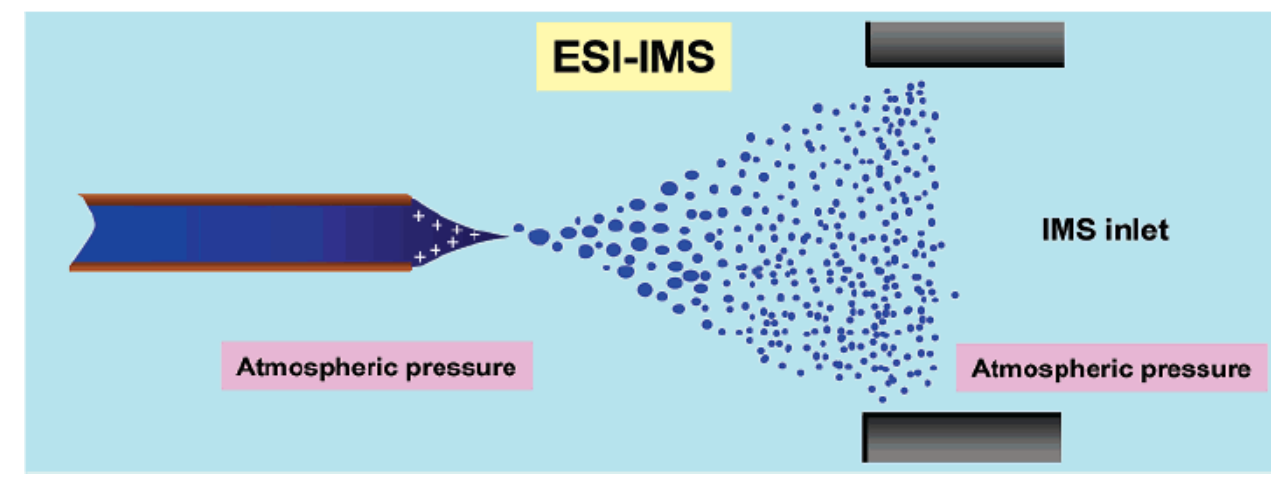

Figure 14: ESI-IMS interface showing solvent being sprayed into IMS [77]

While maintaining the ESI itself at relatively low temperatures prevents premature solvent evaporation which inhibits ionization, the reverse is required once the ions enter the drift tube. As a result of solvent ionization alongside the analyte, the use of mild heating within the drift tube is often required to improve peak resolving power (Figure 15) [74]. 


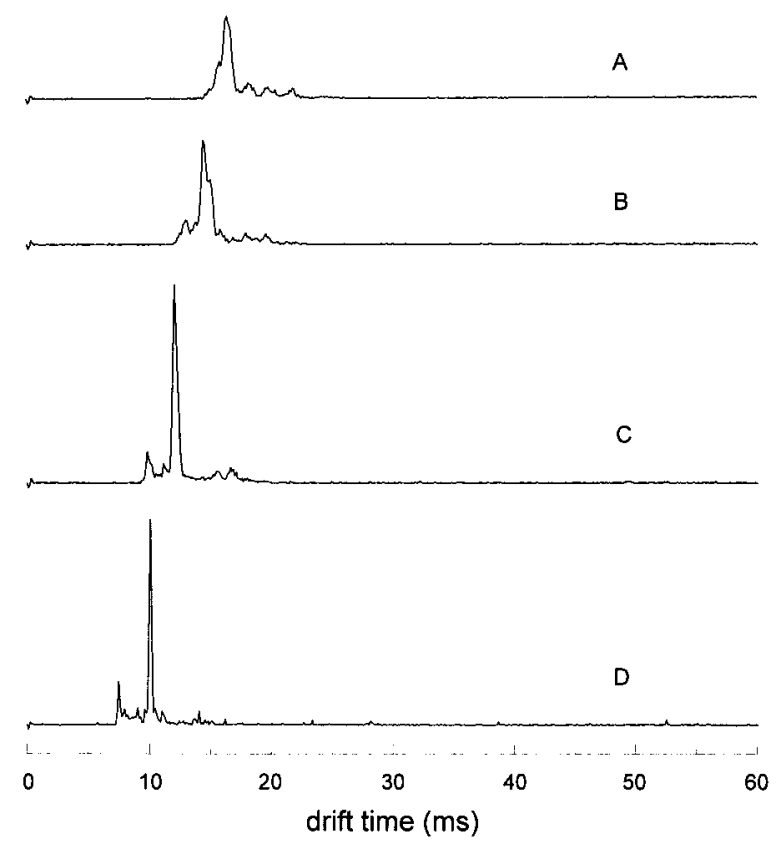

Figure 15: Effect of drift tube temperature on peak resolving power in ESI-IMS at; A-100C, B-150, C-200, D-250C [74]

These are some of the enhancements that can be provided through the utilization of electrospray ionization with ion mobility spectrometry over the use of the traditional radioactive ionization source. It is important to note that these enhancements, higher resolved peaks, simpler to interpret spectra and elimination of the care and handling of a radioactive source have still not propelled IMS within the annals of Analytical Chemistry to be synonymous with GC and LC analysis. Such a transition for IMS still has one obstacle to overcome which stems from a property that is at the same time its greatest strength and now apparently also its weakness. The ability to conduct rapid low cost analyses occurs as IMS performs these analyses in the gas phase under atmospheric conditions. These same atmospheric conditions allows for gas molecules to just as quickly steal and compete with ionized analytes for charge, making the task of analyzing and separating analytes under atmospheric conditions using IMS especially difficult. 


\subsection{Charge Competition in IMS}

On close inspection of an Ion mobility spectra a few things will be observed, the existence of a reactant ion peak (RIP) close to the beginning of the spectra (low drift time) and the analyte peak which is typically further down the spectra (higher drift time).

See Figure 16 and Figure 17.

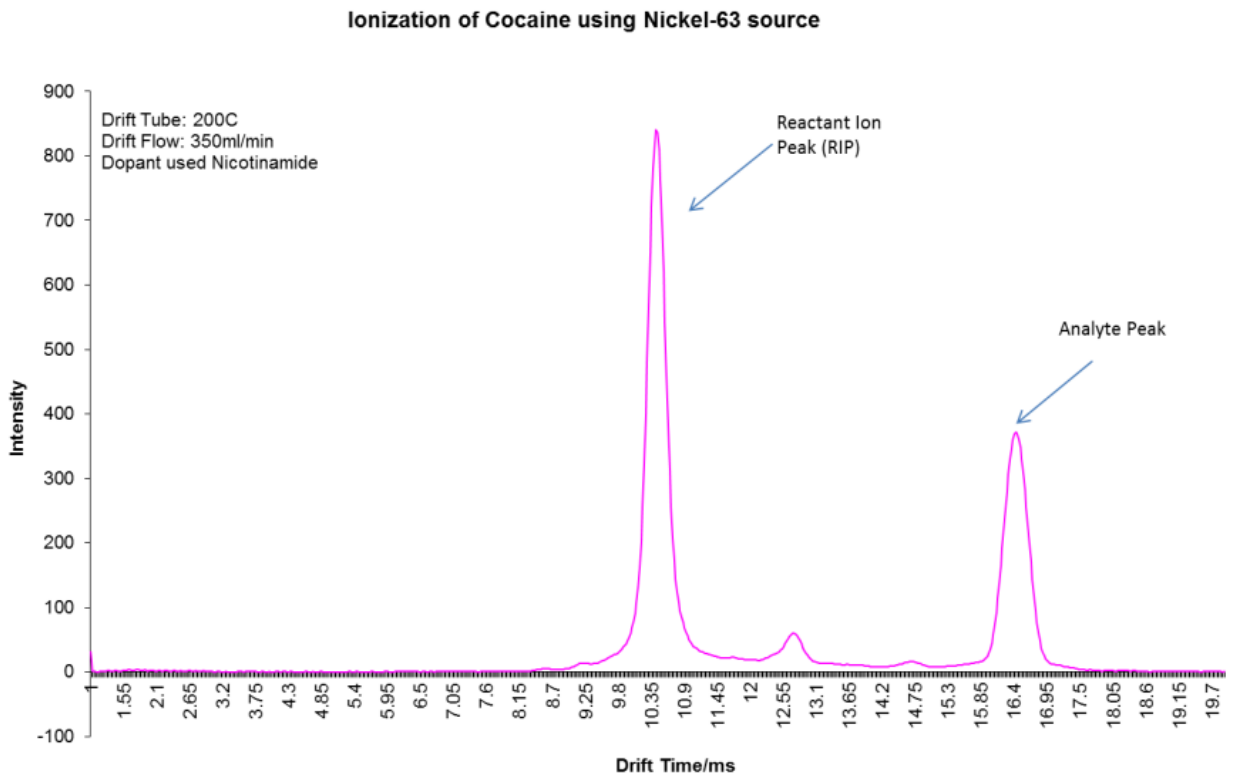

Figure 16: Ionization of Cocaine using Nickel 63 source 


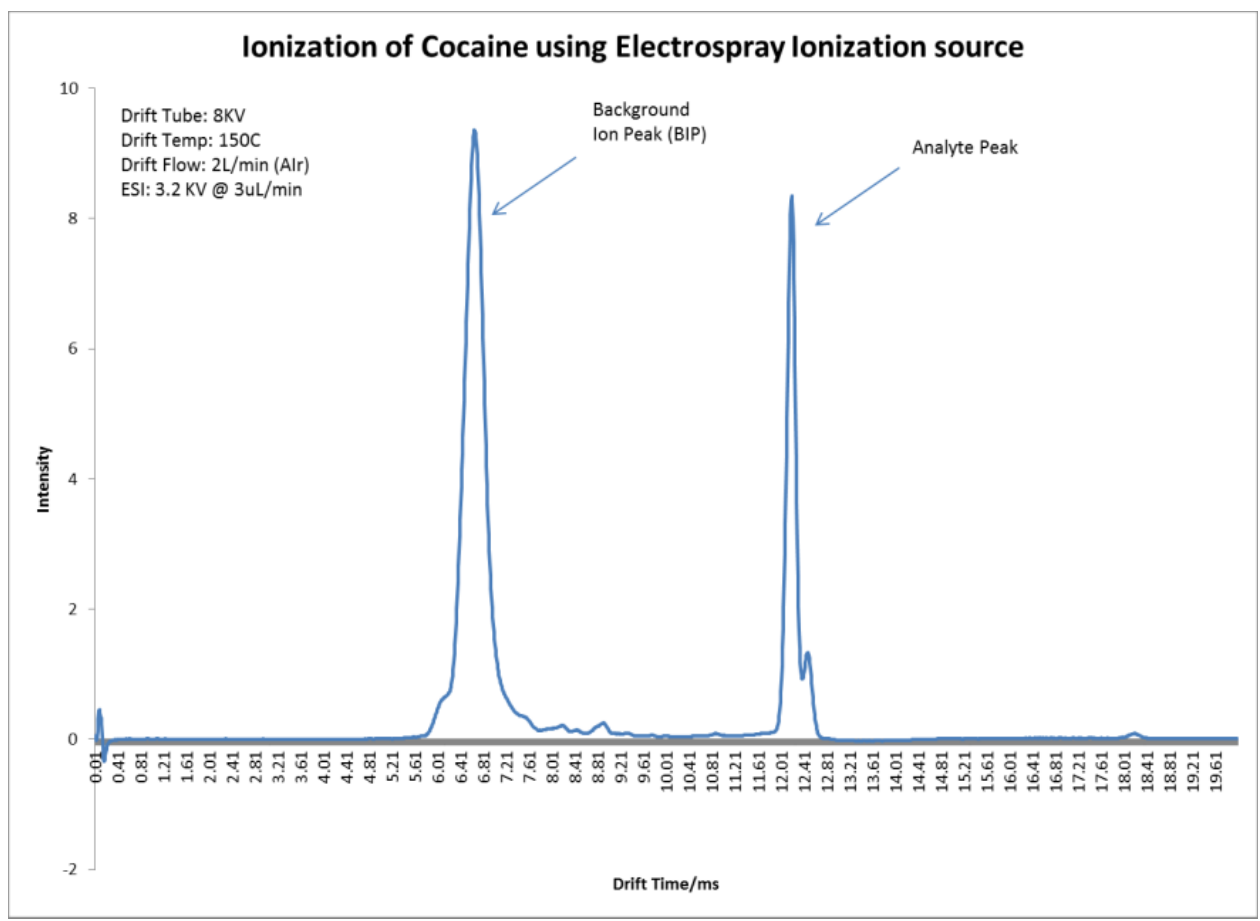

Figure 17: Ionization of Cocaine using Electrospray Ionization

These peaks observed in addition to the analytes termed reactant ion peak (RIP) for traditional radioactive sourced IMS units and background ion peak (BIP) when referring to electrospray ionization [79], are unavoidable. In fact, for many IMS applications, these peaks are manipulated to minimize the observation of other unwanted peaks that may be within the supporting drift gas. The figure below captured from Gary Eiceman's magnum opus "Ion Mobility Spectrometry" depicts the hypothetical concept of selecting an appropriate reagent gas to produce a RIP in an attempt to reduce the observance of unwanted peaks within the mobility spectra (Figure 18). 


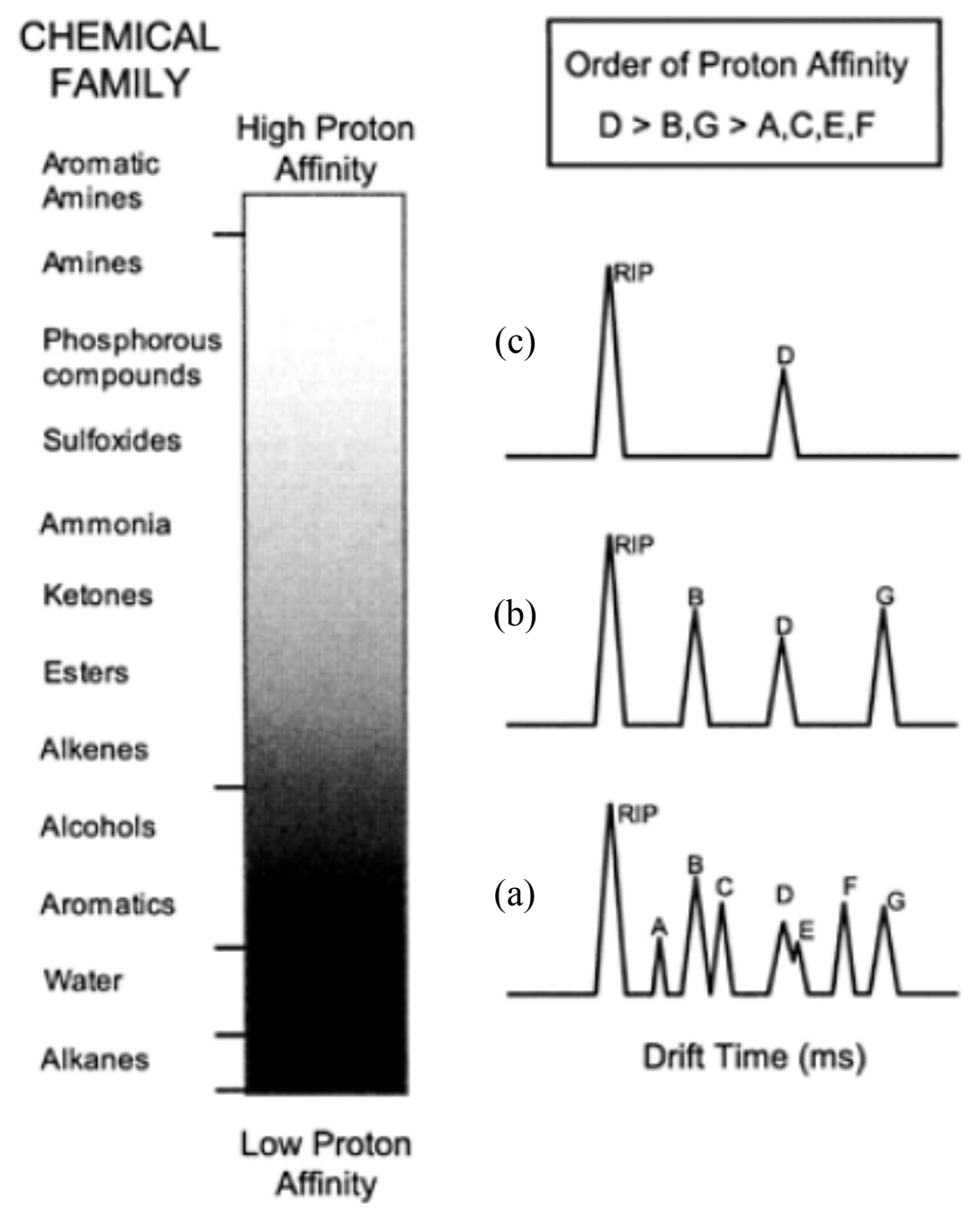

Figure 18: Hypothetical example of selecting dopant to eliminate unwanted peaks [1].

The figure depicts the effect that selecting a RIP with a particular proton affinity will have on the spectra of a group of analytes being ionized. During the ionization process, the analytes with the highest proton affinity will be ionized preferentially in the presence of those species of a lower proton affinity. As a result, a RIP that is selected with a particular proton affinity will suppress the ionization of analytes that have a lower proton affinity. As a result of this suppression effect, those analytes are not ionized and so not observed. Consider Figure 18 (a), where a RIP with a low proton affinity is used, analytes 
A,C,E,F,B \& $\mathrm{G}$ are all observed during ionization as they possess a proton affinity greater than RIP (a). Analysis shown in Figure 18 (c) reveals the use of a RIP with a much higher proton affinity than analytes $\mathrm{A}, \mathrm{C}, \mathrm{E}, \mathrm{F}, \mathrm{B} \& \mathrm{G}$ resulting in their ionization being suppressed and those analytes not being observed despite them being present. Haphazard use of IMS for analysis without careful consideration for analyte proton affinities often leads to either noisy spectra from the ionization of too many analytes or results in missed information that stems from the suppression of ionization of expected analytes. Depending on the ionization source being used and the ionization energy of those sources, the spectra obtained during IMS analysis can be significantly different for analysis of the same analyte owing to simultaneous ionization of unwanted compounds by different sources (Figure 19).

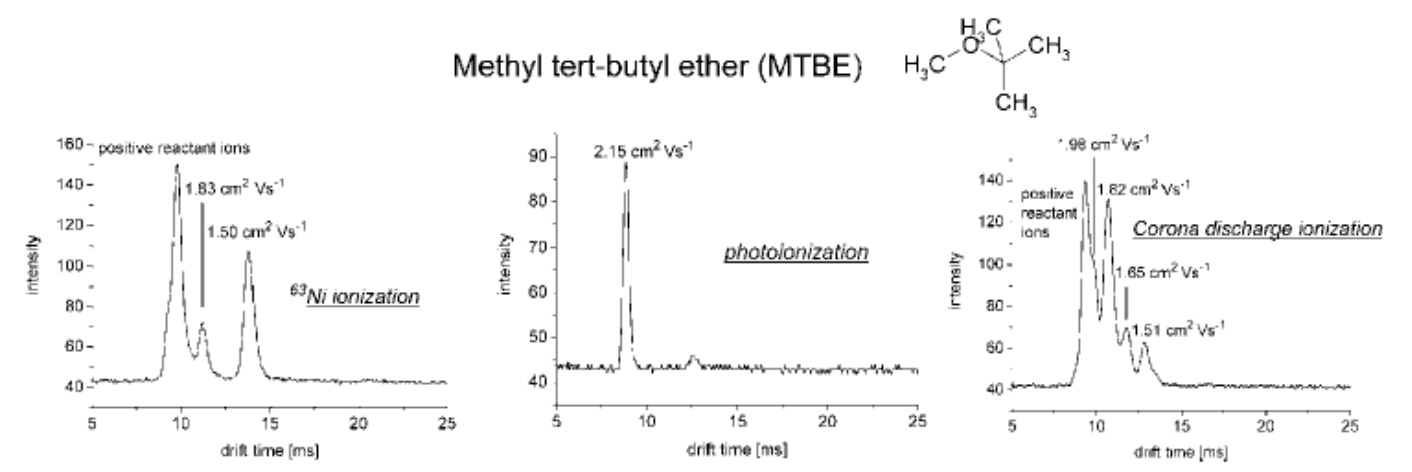

Figure 19: Various ion mobility spectra showing interferences from surrounding atmosphere [67].

The selection of a RIP is relatively straightforward for ionization sources that operate by analyzing a gaseous sample. To obtain a particular RIP under these circumstances, the target compound is "doped" into the drift gas via a permeation device. The doped drift gas will then contain trace amounts of the selected compound, for example ammonia. The doped drift gas will be ionized by the ionization source and in turn ionize or suppress 
analytes depending on proton affinity of the dopant. For negative ionization, the selection criterion is based on the electron affinity of the dopant, as negative ion mode produces negative ion species.

In electrospray ionization, dopants are not used as the ionization of analytes takes place within solution prior to the ions entering into the gas phase and interacting with the drift gas. Therefore a different approach is adopted for ionization/suppression criteria within ESI. Selected additives, sometimes called modifiers, are mixed with the ESI solvent in an attempt to determine the type of ionic species generated/suppressed. Previous studies focusing on electrospray ionization coupled to mass spectrometry outside the field of Ion mobility, have revealed that the addition of acids to ESI solvents facilitate ionization when using positive ion mode, while halogenated solvents facilitate the production of analyte anions by proton abstraction or halogen attachment in negative ion mode [80]. Anecdotal evidence is observed throughout reported literature that indicates a relationship between the amount of modifier added to ESI solvents and the relative intensity of a particular analyte [81-85]. One of the major efforts of the present body of work is to highlight the relationship between modifiers within the ESI solvent and the analyte to determine how best to minimize ion suppression effects when utilizing ESI, as there has been no direct or conclusive works previously published on this particular topic.

Compounding this is the difference of ion behavior in a liquid phase versus that behavior in the gas phase. Liquid and gas phase basicity was found to vary greatly for some analytes, resulting in analytes being preferentially ionized while in solution but then losing their charge to another more basic gas phase species. Overcoming this particular 
obstacle, though mountainous, was attempted and the results will be discussed and has also been published in peer reviewed literature [86].

\subsection{Calculating Reduced Mobility in IMS experiments}

No writing on ion mobility may be complete without mentioning reduced mobility values. As indicted in the section on Overview of Ion Mobility Spectrometry (pg. 15), the ion swarm migrates down the drift tube under the influence of an applied electric field. The velocity of this swarm approximated from the rate of travel within the length of the drift tube, is directly proportional to the intensity of the applied electric field, the higher the electric field (E), the higher the velocity (v) (Equation 1)

\section{Equation 1: Relationship of ion drift velocity to electric field strength}

$$
v \propto E
$$

Assuming a constant relationship between ion velocity and electric field, the equation becomes.

Equation 6: Including mobility constant to correlate ion velocity and electric field strength [1]

$$
v=K E
$$

Where the constant $K$ is termed the ion's mobility, which is plotted versus time to produce an Ion Mobility Spectrum. Rearrangement of the equation produces:

$$
\begin{aligned}
& \text { Equation 7: Rearrangement of mobility constant to include the length of the drift tube } \\
& \qquad K=\frac{v}{E}=\left(\frac{d}{t_{d}}\right) \cdot\left(\frac{1}{E}\right)=\frac{d}{t_{d} E}
\end{aligned}
$$

Equation 7 shows that the ion velocity (v) may be expended to be a function of (d) the distance travelled by the ions which is proportional to the length of the drift tube, divided 
by the time taken by the ions to travel that length which is proportional to the drift time $\left(t_{d}\right)$. The tendency for different ions to migrate down the electric field at different rates provides separation, as these ions are said to have different mobility values (K). Mobility is also dependent on the temperature and pressure of the drift tube in which the ions migrate and so the mobility is often corrected to standard temperature (273 Kelvin) and Pressure (760 Torr) to produce a reduced mobility value $\mathrm{K}_{0}$.

Equation 8: Relating reduced mobility to mobility [1]

$$
K_{0}=K \cdot\left(\frac{273}{T}\right) \cdot\left(\frac{P}{760}\right)
$$

Equation 9: Expanded reduce mobility equation [1]
\[ K_{0}=\left(\frac{d}{t_{d} E}\right) \cdot\left(\frac{273}{T}\right) \cdot\left(\frac{P}{760}\right) \]

The equation for reduced mobility as described is therefore not affected by temperature and pressure. The reduced mobility value is assumed constant for a particular ion (analyte) once factors of analytical pressure and temperature are taken into consideration, this allows a reduced mobility value to be used as an identification marker for a particular analyte. A comparison of reduced mobilities can also be carried out by relating the unknown reduced mobility to that of a standard with known reduced mobility (Equation $10)$.

Equation 10: Relationship between reduced mobility values of two analytes $[1 ; 87]$.

$$
\frac{K_{0(\text { unknown })}}{K_{0(\text { standard })}}=\frac{t_{d(\text { standard })}}{t_{d(\text { unknown })}}
$$

Equation 10 has generally been the equation used during ion mobility experiments to determine the reduced mobility values of unknown analytes compared with that of a 
standard. For example, a compound such as 2,4 Lutidine with a known $\mathrm{K}_{0}$ of $1.95 \mathrm{~cm}^{2} \mathrm{~V}^{-1}$ [88] may be analyzed on an instrument and then used to determine $\mathrm{K}_{0}$ values of other analytes analyzed on the same instrument with the same conditions by substituting the drift time values obtained for 2,4 Lutidine and the known analyte into Equation 10. Identification of compounds through comparison of reduced mobility values has proven to be a reliable method for IMS. The technique of using reduced mobility is not limited to the use of standard compounds; the comparison can also be done by using the $\mathrm{K}_{0}$ of RIP within the spectra which has also been reported to produce very precise and accurate results [89]. Various publications have reported tables of $\mathrm{K}_{0}$ values for hundreds of compounds, a quick comparison of the determined $\mathrm{K}_{0}$ from Equation 10 to that from published tables has been tested to produce reliable identification of unknowns [70;90]. While the determination of an unknown via comparison to a standard using Equation 10 is beneficial, the initial determination of an ions mobility value $\mathrm{K}$ is in fact more complex. Equation 7 shows the relationship that knowledge of drift tube length in $\mathrm{cm}(\mathrm{d})$, drift time in seconds (td) and electric field strength in volts per centimeter (E) will produce a value for $\mathrm{K}$. While this would be true for ions travelling in a vacuum, this is not the case for ions travelling under atmospheric pressure. To correct for these factors, the value for $\mathrm{K}$ is expanded to include other factors such as the density of the drift gas $(\mathrm{N})$, the columbic charge on the ion $(\mathrm{q})$, the effective mass of the ion $(\mu)$, the Boltzmann constant $(\mathrm{k})$, the temperature of the drift gas $(\mathrm{T})$ and the effective collision cross sectional area of the ion $(\Omega)$ (see Equation 11). 


\section{Equation 11: Expanded ion mobility equation [1].}

$$
K=\left(\frac{3 q}{16 N}\right)\left(\frac{2 \pi}{\mu k T}\right)^{\frac{1}{2}} \frac{1}{\Omega_{D}}
$$

The relationship between $\mathrm{K}$ and $\Omega$ is what allows IMS to differentiate analytes based on their shape to charge ratio. The relationship only holds true at certain values for $E$ and $N$ termed the field value or Townsends [91]. Low field conditions of IMS occur at 200 to $400 \mathrm{~V} / \mathrm{cm}$ of applied electric field strength across the drift tube. The application of stronger electric fields (E) will drive the ions across the drift tube at a faster rate, by increasing the velocity of the ion $(v)$ and ultimately its mobility $(K)$ as seen in Equation 6. Any increase in ion velocity also results in more collisions between the ion and the surrounding drift gas, these collisions effectively slows down the ion. The increased collisions makes necessary the consideration of drift gas density $(N)$ in addition to $(E)$ when describing the overall electric field strength (Equation 12).

Equation 12: Relationship between field condition, field strength and gas density [1] Townsend $(T d) \propto E / N$

As a result of the relationship shown in Equation 12 referred to as the Einstein equation, low field conditions in IMS should be described as $\leq 6 \mathrm{Td}$ [92]. Under these field conditions Equation 11 holds true. Field conditions above these values are typically used to conduct Field Asymmetric Ion Mobility Spectrometry (FAIMS) or the older termed Differential Mobility Spectrometry (DMS). These techniques provide separation determined by differences in an ion's mobility defined by a tendency of the ion's mobility to change under high applied field strength. Differential mobility devices operate outside the field range of traditional linear drift tube IMS field units and so the 
equations that govern their separation are different, allowing them to deliver superior resolving capabilities for various applications (Equation 13).

Equation 13: Coefficient of mobility in a high electric field [92]

$$
K(E / N)=K_{0}\left(1+\sum_{n=1}^{\infty} \propto_{2 n}\left(\frac{E}{N}\right)^{2 n}\right)
$$

Where $\mathrm{K}_{0}$ is the reduced mobility under low field conditions and $\alpha_{2 \mathrm{n}}$ are the coefficients preceding the powers of $\mathrm{E} / \mathrm{N}$ in the equation. As a result, mobility $(K)$ does not remain constant at high field values ( $\geq 6 \mathrm{Td}$ ), this behavior is exploited by differential mobility devices to allow for separation of ions that possess the same or similar mobilities under low field conditions. Figure 20 shows the alpha values of a group of analytes that have similar coefficients at low field strengths $(\leq 6 \mathrm{Td})$ but shows large differences as the field strength is increased, these differences in $\alpha$ allows for the separation of the analytes according to Equation 13. The reason for this change in mobility under high field conditions is believed to be as a result of thermal energy being transferred to the ions resulting in ions having much higher kinetic energy. Full reviews of differential mobility may be found within reported literature [67;93-101], but was not utilized during this research project thereby limiting further discussion of details of the technique. 


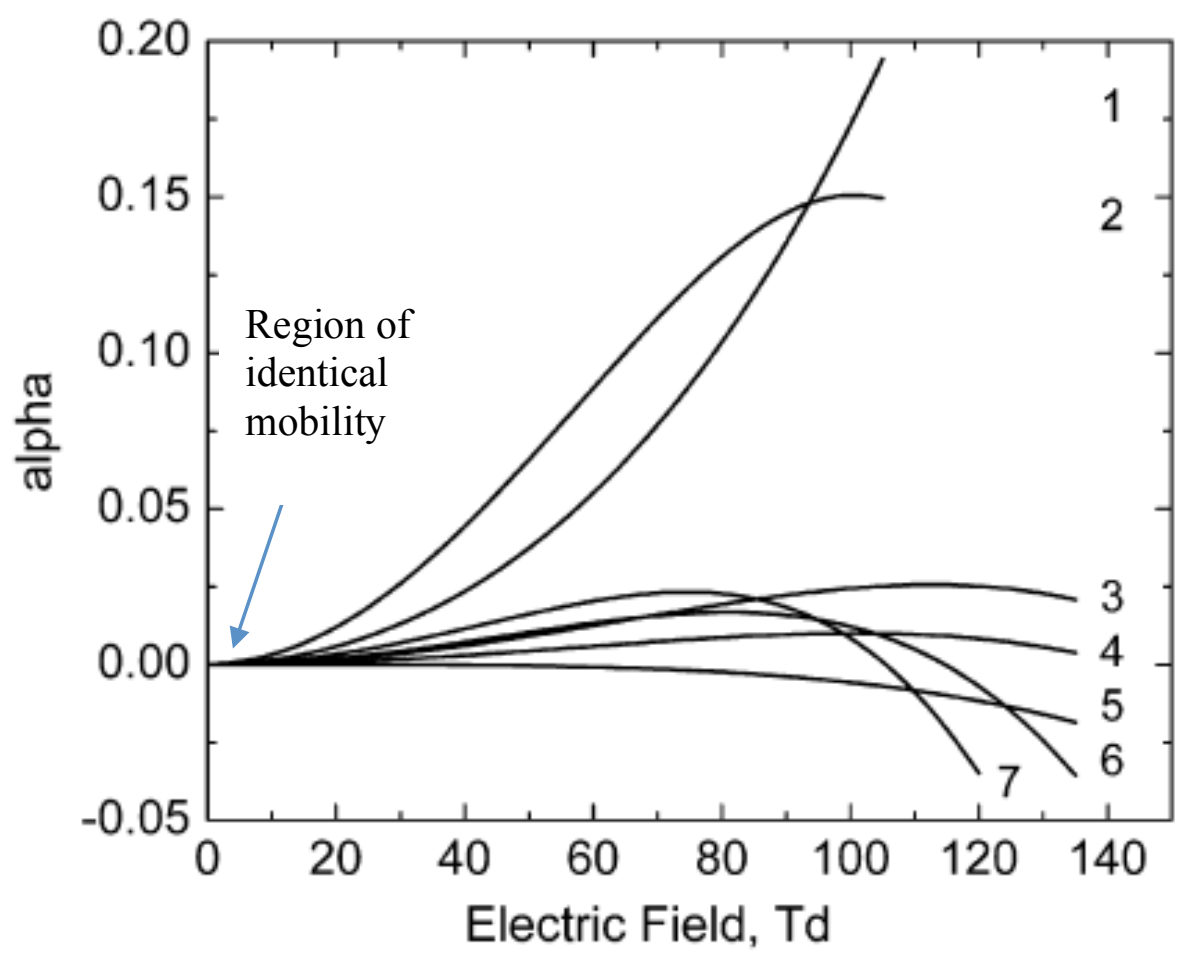

Figure 20: Alpha vs. Electric field for a group of analytes in differential mobility [93]

Instrument manufacturers of low field linear drift tube IMS units such as Smith's detection, have developed software that incorporates values such as Equation 10, into its IMS data analysis algorithm. The software allows the instrument to perform on the fly analysis of unknowns and instantaneously generate a match termed an "Alarm" to a predetermined set of reduced mobility values of analytes. The only requirement being a periodic calibration with a reference standard to compensate for any deviations in the mobility values that might occur over time. These deviations typically occur as a result of factors such as increased moisture within the drift gas which tends to cause greater formation of adducts around ions increasing both the effective mass of the ion $(u)$ and the collisional cross sectional area $(\Omega)$. An increase in both these terms reduces the mobility $(K)$ as seen in Equation 11. However, the effect is systematic as all ions are separated 
within the same drift tube, so compensations made for observed mobility changes of one ion typically translates into the same change for any other ion within those same conditions. These changes still allow the use of Equation 10 in determining unknowns by the use of a standard with a known $(K)$ despite observed changes in mobility because of atmospheric influences.

Atmospheric influence segues into another crucial requirement when conducting IMS analysis under ambient conditions, since ambient conditions vary by location. For example, IMS experiments conducted in areas with low relative humidity and low barometric pressure will produce ion mobility spectra with very different mobility values from those experiments conducted under the same instrument parameters but in a location with much higher relative humidity and higher barometric pressure. To compensate for these differences the reduced mobility $\left(\mathrm{K}_{0}\right)$ is used instead of mobility (K) (Equation 8). In theory the reduced mobility of an analyte is assumed to be constant at low field strength, hence its use as an identification tool. However, Eiceman has reported that not all analytes behave in this manner [87]. A 2003 study revealed that the reduced mobility of some analytes are greatly affected by changes in temperature Figure 19, this could lead to misidentification of analytes under certain conditions. 


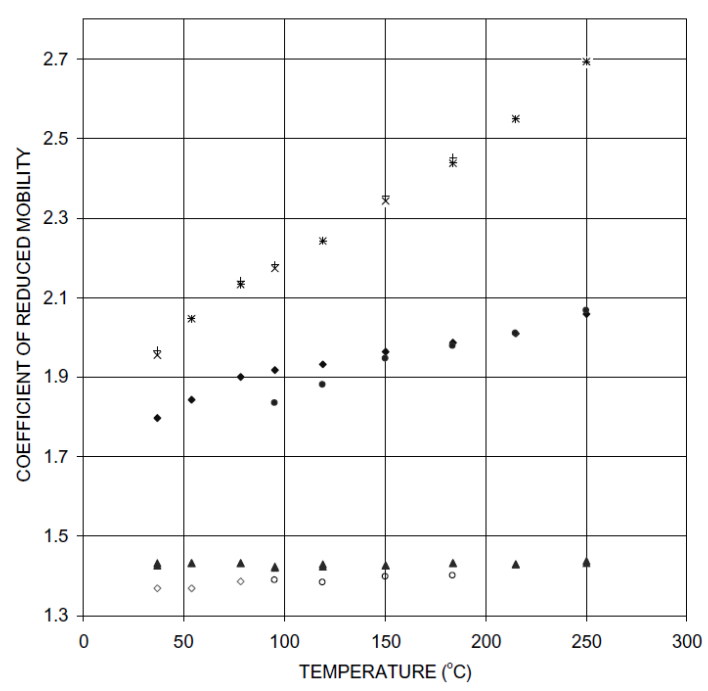

Figure 21: Effect of temperature on calculated reduced mobility values of four analytes[87]

A later study by Hill et al. revealed that contaminants in the drift gas may also affect the calculated reduced mobilities of analytes [88] again raising a cautionary flag when conducting experiments and comparing results across platforms involving different locations and different instruments. On a hold however, once precautions are taken to control pressure, temperature, moisture and drift gas purity, IMS has been proven to provide trustworthy data. In fact no other technique has been developed that combines the speed of analysis offered by IMS with comparable levels of detection, reliability and low cost of operation. 


\section{MATERIALS AND METHODS}

All chemicals and reagents used were of spectrometric grade. ( $R$ \& S) Cathinone, ( $R$ \& S) Methamphetamine, (RS \& SR) Ephedrine and (SS \& RR) Pseudoephedrine where purchased from Cerilliant Corp, Round Rock, Texas. S-2-(+) Sec Butanol, (D \& L) Methionine, Hexyl alcohol (1-Hexanol) and 1-Heptanol were obtained from Acros Chemicals and purchased through Fisher Scientific Corporation, Pittsburgh Pennsylvania USA. Methanol, 1-Propanol, 1-Butanol and 1-Pentanol were supplied by Fisher Scientific. Ethanol and 1-Octanol were purchased from Sigma Aldrich Corporation, St. Louis, Missouri. The electrospray ionization (ESI) source was maintained at $+3.2 \mathrm{KV}$ above the Drift Tube potential for positive ion mode and $-2.8 \mathrm{KV}$ below the drift tube for negative ion mode analysis. The Drift Tube was maintained at $+8 \mathrm{KV}$ for positive ion mode and $-10 \mathrm{KV}$ for negative ion mode at a temperature of $150^{\circ} \mathrm{C}$, drift flow was $2 \mathrm{~L} / \mathrm{min}$ of ultra-high purity (UHP) air from Airgas, Miami, FL. The modifiers were infused at $4 \mu \mathrm{L} / \mathrm{min}$ and had an independently controlled carrier flow of purified air at $800 \mathrm{ml} / \mathrm{min}$ into the drift region. The modifier was infused using a Harvard Apparatus Model 11 syringe pump while the ESI supply was infused with a Harvard Apparatus Model 22 syringe pump from Holliston, Massachusetts. The modifier syringe pump was fitted with a $250 \mu \mathrm{L}$ Hamilton Gas tight syringe while the ESI syringe pump was fitted with a $50 \mu \mathrm{L}$ Hamilton Gas tight syringe from the Hamilton Company, Reno, NV. Modifier and ESI liquids were infused through a $350 \mu \mathrm{m}$ OD/75 $\mu \mathrm{m}$ ID neutral capillary tube from Polymicro Molex, Lisle, Illinois. Samples were filtered using a $0.2 \mu \mathrm{m}$ hydrophobic polytetrafluoroethylene based filter from Millex Millipore, purchased through Fisher Scientific Corporation, Pittsburgh Pennsylvania. The ESI-CIMS-MS (model RA4100) 
was designed and built by Excellims Corp, Acton Massachusetts. The instrument was coupled to an Extrel QC-150 quadrupole with mass range 10 to 500amu, Extrel CMS, Pittsburgh, Pennsylvania. Both IMS and quadrupole may be operated in either negative or positive mode. One nickel-63 IMS system used was a Barringer Ionscan 400B from Smith's Detection, Smith's Group plc, UK. Fitted with Nicotinamide (CAS 98-92-0) as the positive mode dopant and hexachloroethane (CAS 67-72-1) as the negative mode dopant. The other nickel-63 based IMS system was a GE Itemizer 2 from Safran Morpho, Morpho Detection, Wilmington MA. Solid Phase Microextraction (SPME) fibers used were coated with a $100 \mu \mathrm{m}$ polydimethylsiloxane from Sigma Aldrich Corporation St. Louis, Missouri.

Graphic artwork was rendered by Google SketchUp v 8.0 Google Inc. All molecules were rendered by Chemsketch v. 12.01 ACD Labs (Freeware version). Statistical modelling was done utilizing JMP version 10.0.2 from SAS. Density Functional Theory (DFT) quantum chemical calculations were performed at the B3LYP/6-31g level of theory using the Gaussian 09 program suite. 


\section{EXPERIMENTAL DESIGN}

\subsection{Adaptation of an Ion Mobility Spectrometer for Chiral Separations}

Experiments for this research project began by utilizing a commercially available Ion Mobility Unit designed and built by Smith's Detection, Smith's Group plc, UK. The unit used was a Barringer Ionscan 400B IMS unit (Figure 22) which is identical to the Smith's Ionscan 400B model. Barringer was acquired by Smith's detection in 2001, and Smith's continued marketing Barringer's IMS trace detection instruments under the new name Smith's Ionscan 400B [102].

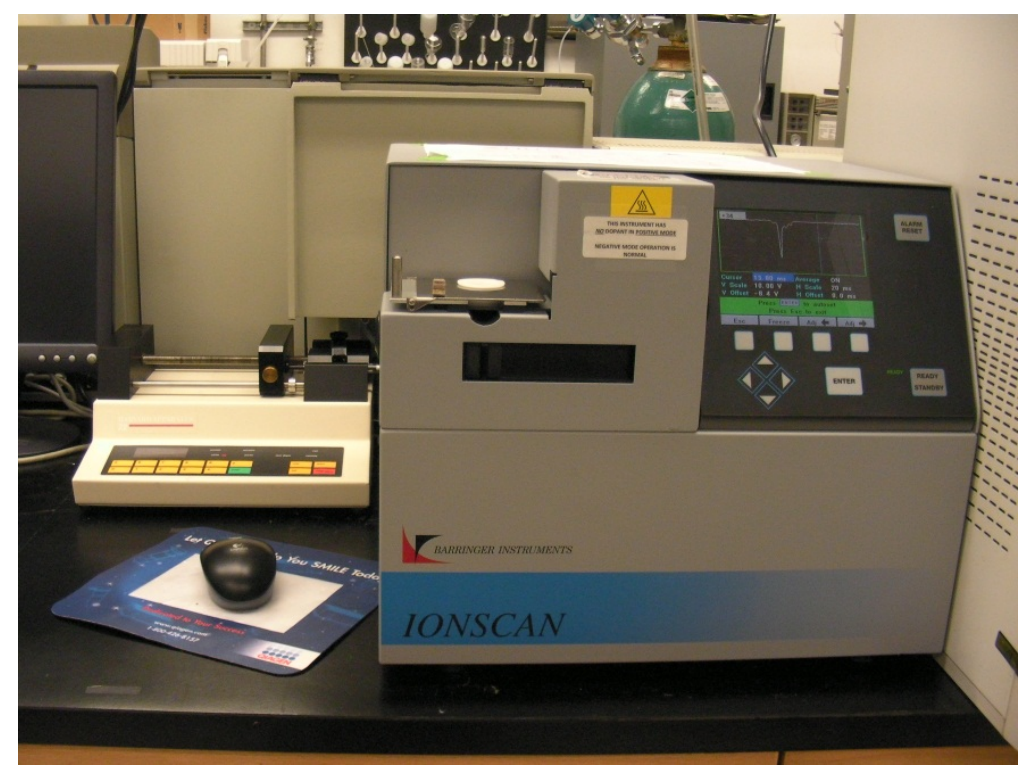

Figure 22: Barringer Ionscan 400B

The advantages of using a commercially available OTS instrument were numerous, well integrated hardware and software and a simplified user interface. However, the disadvantages were equally numerous and at times very prohibitive towards advanced analytical work. The result of well integrated parts inhibited the alterations to many of these components, which was the case when attempting to perform chiral separations. The Barringer 400B utilized a 10 milliCurrie ${ }^{63} \mathrm{Ni}$ ionization source that was coupled to a 
drift tube of approximately $7 \mathrm{~cm}$ in length. Samples were loaded onto a sampling port where they were introduced via thermal desorption unto the ionization region of the IMS by a heated anvil (Figure 23). The desorbed analyte interacted with the reactant ion peak to form analyte ions which were separated and identified on the basis of reduced mobility values. The overly simplified operation has resulted in over 50,000 similar IMS unites sold by companies like Smith's detection being used at airports and security checkpoints globally [103]. Samples are loaded onto the sample port through wipes of suspect articles using a round sorbent that is generally but not always cellulose based.

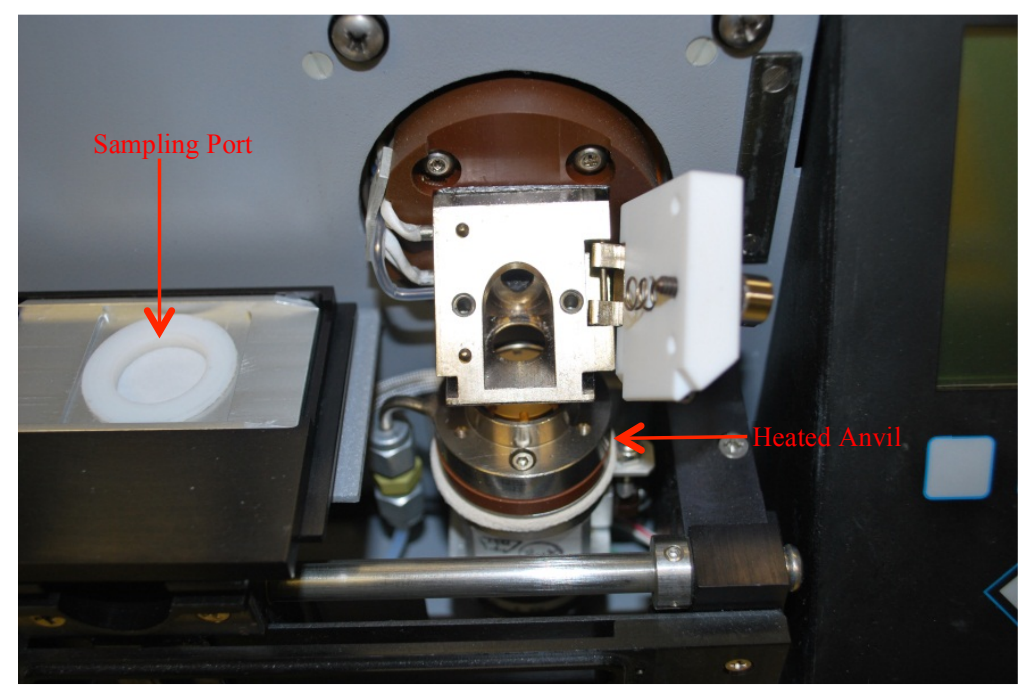

Figure 23: Sampling port of Ionscan 400B

While this instrument performs well for screening applications, its limitations are quickly revealed within the laboratory. An inability to analyze multiple analytes is the primary barrier to true analytical work as well as an inability to adjust parameters to suite a wider array of analytes. Parameters such as the drift tube potential, wider control of drift tube temperature and various software controls restrict instrument use outside factory preset parameters. An inability to perform analysis once RIP peak width is outside pre- 
determined values was one such condition that prevented analytical work outside the instruments factory determined operating range. One of the largest obstacles to the full utilization of this instrument for analytical work was also a difficulty in utilizing different dopants to form different RIP's. The instrument was preloaded with Nicotinamide (CAS 98-92-0) as the dopant for positive ion mode and Hexachloroethane (CAS 67-72-1) as the dopant for negative ion mode. The ability to limit or suppress ionization of analytes rests with the ability to manipulate the RIP. As a result, changes in the instrument's RIP was the first parameter to be altered despite a lack of instructions from the instrument manufacturer on how to do so. The dopants for the Ionscan 400B are located in heated aluminum chambers on the left side of the instrument drift tube behind the high voltage supply module (Figure 24).

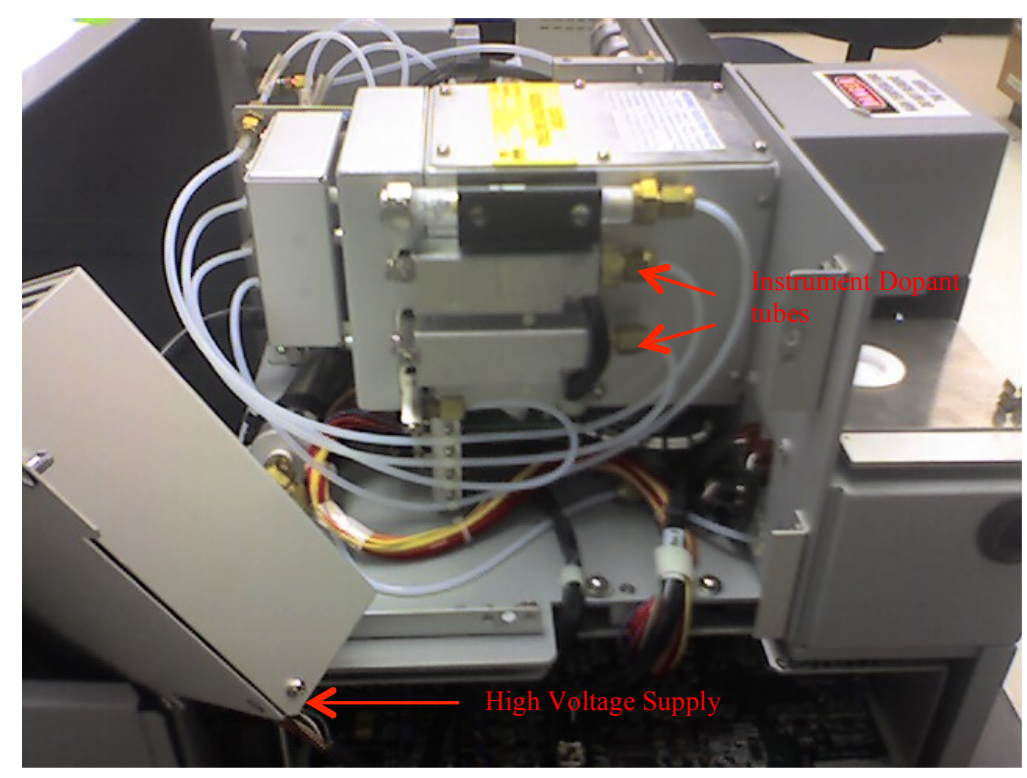

Figure 24: Location of Dopant on Ionscan 400B

Once the dopant tubes were located, simple tools were used (wrench and screwdriver) to open the chambers which allowed removal of both dopants. The dopants themselves should be handled with care and kept clean, this will allow the dopants to be re-used in 
the future if desired. For experiments involving low proton affinity analytes, no dopants were used within the Ionscan 400B. The removal of the dopant resulted in the formation of protonated water clusters from air. The lack of dopant resulted in an ability to analyze a wide spectrum of analytes from low to high proton affinity, despite the fact that the Ionscan 400B was not originally designed to conduct these analyses. Amphetamine Type Substances (ATS) were the first compounds analyzed in order to evaluate their response within the Ionscan 400B in the positive ion mode. The following compounds and their enantiomers were analyzed; Methamphetamine, Cathinone, Ephedrine and Pseudoephedrine. These compounds were sourced from Cerilliant, Round Rock Texas USA and the reagents used for dilution spectrometric grade. All analytes were diluted in methanol for these tests. The first task in analyzing new substances on the Ionscan 400B was to add the mobility values of these substances into the software to allow for their identification. Analysis of samples however, was made slightly difficult through the removal of the standard nicotinamide dopant to which the software references all other mobility values by using Equation 10. Since the RIP was no longer nicotinamide with a reduced mobility value of $1.86 \mathrm{~cm}^{2} \mathrm{~V}^{-1} \mathrm{~s}^{-1}$ but of Air with a reduced mobility value of 2.31 $\mathrm{cm}^{2} \mathrm{~V}^{-1} \mathrm{~s}^{-1}[104 ; 105]$, the reduced mobility value of air had to first be programmed as the new reduced mobility of the calibrant within the Ionscan 400B software. 

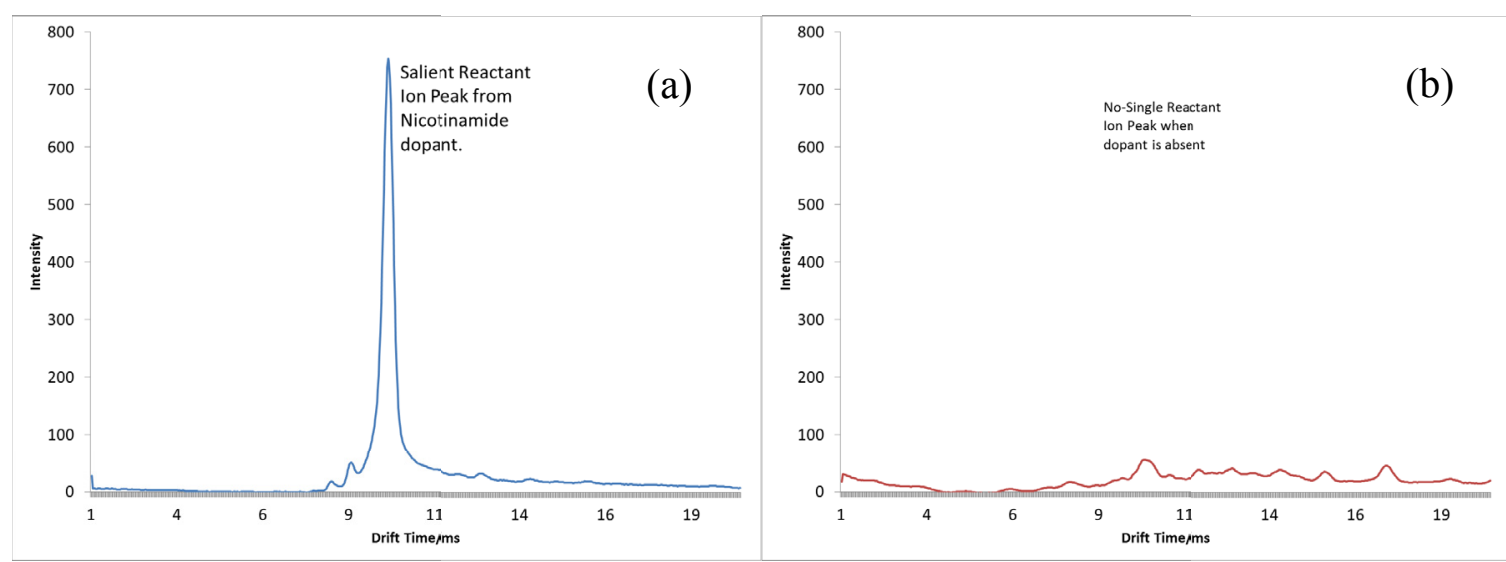

Figure 25: Comparison of RIP with (a) Nicotinamide and (b) Air

As can be seen from Figure 25, the use of air did not display a salient RIP and displayed nosier spectra when compared with the use of nicotinamide as the RIP. After reprogramming and re-calibrating the $400 \mathrm{~B}$ software, $1 \mu \mathrm{L}$ of traditional standards Cocaine and Diazepam, diluted to $10 \mathrm{ng} / \mu \mathrm{L}$ were analyzed to ensure that the instrument was still able to detect and identify these substances. Next was the addition of the drift times of the new amphetamine type substances into the 400B software. By individual analysis of each analyte, the drift time was obtained. Once the drift time was obtained the reduced mobility vale is calculated by utilizing Equation 10, this mobility value is programmed within the $400 \mathrm{~B}$ software to determine the presence of these analytes from unknown samples. Despite the seemingly poorer spectra obtained by utilizing air as the reactant peak, it provided the advantage of not suppressing analytes of interest as well as having a small enough reactant ion peak that would not interfere with chiral separation experiments as these experiments caused peaks to shift significantly along the spectra and would at times interfere with the very prominent RIP obtained from using Nicotinamide. The ability to change the dopant within the OTS Ionscan 400B removed one of the 
largest obstacles to utilize the instrument to perform a wide array of analysis. Additional modifications were still required in order to conduct chiral separations on the Barringer 400B. In 2006 Prabha Dwivedi reported on the ability to perform gas phase chiral separations by ion mobility [12]. The separation entailed adding a chiral "modifier" to the IMS drift gas that preferentially interact with one of a pair of enantiomers more than the other, resulting in separation of the enantiomeric compounds. In order to replicate these findings, the previous experiments as reported by Dwivedi et al. were attempted on the Barringer 400B. Modifications were carried out that would allow the addition of an additional compound into the drift gas of the Ionscan 400B. The compound or modifier, was S-2-Butanol as described by Dwivedi et al., is a chiral compound that was reported to interact with other chiral compounds preferentially to effect separation. The amount of the compound reported to effect the separation was 10 parts per million of S-2-Butanol within the drift gas. With the drift gas at a flow rate of $300 \mathrm{ml}$ per minute, this equated to a modifier effusion rate of $45 \mu \mathrm{L}$ per hour of S-2-Butanol. Delivery of S-2-Butanol was attempted by utilizing a permeation tube obtained from VICI Metronics. These blank permeation tubes are filled with the analyte of choice and then tested gravimetrically to determine the actual permeation rate of the analyte under experimental conditions. These tests with S-2-Butanol revealed a permeation rate of $35 \mu \mathrm{L}$ per hour (Figure 26). 


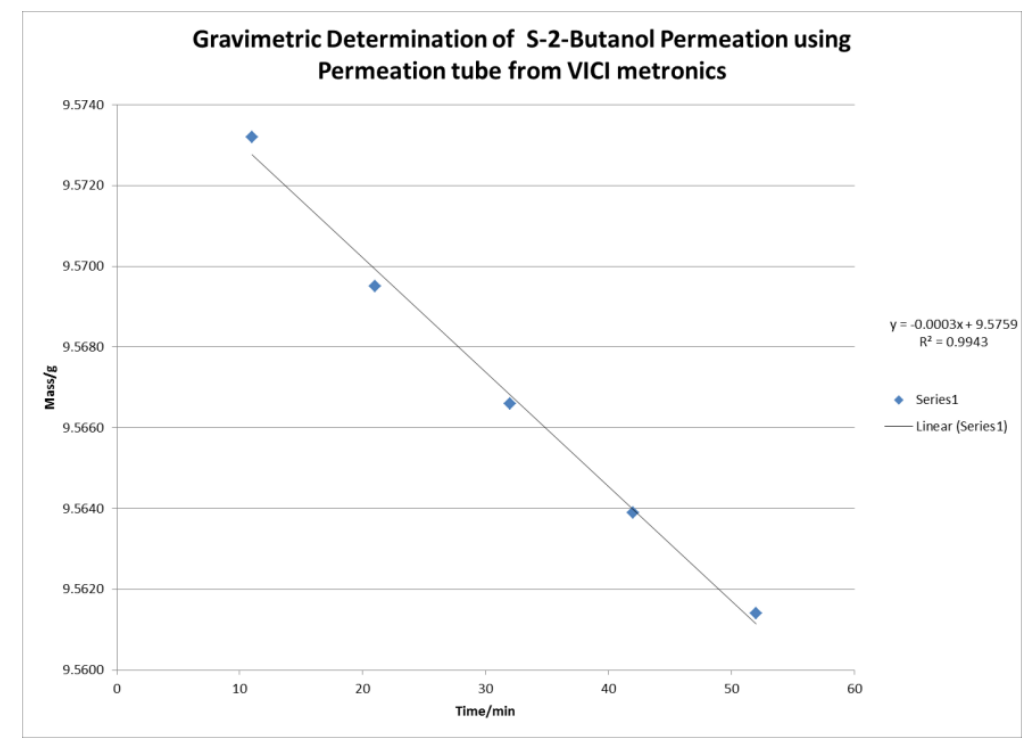

Figure 26: Gravimetric determination of S-2-Butanol permeation rate

To deliver this S-2-Butanol, the permeation tube was placed within an independently installed dopant chamber borrowed from a GE Itemiser 2 which delivered the vapors into the Ionscan 400B IMS drift tube (Figure 27). The S-2-Butanol permeation tube was unable to be fitted in the standard Ionscan 400B dopant chamber shown in Figure 24.

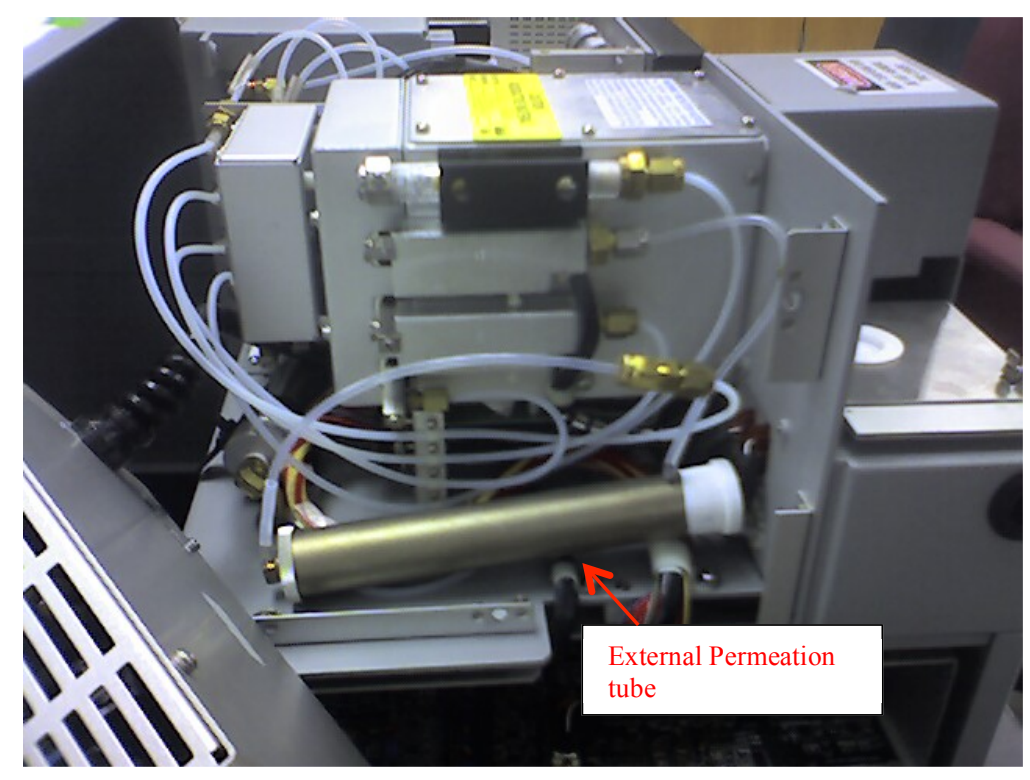

Figure 27: Image showing external dopant tube used for S-2-Butanol permeation 
Results from these modifications revealed a change within the IMS spectra, in addition to the peak for air, an additional peak was also observed believed to be the result of S-2Butanol also being ionized by the ${ }^{63} \mathrm{Ni}$ beta particles (Figure 28). Analytes detected through this arrangement showed good signal response, however the expected shift in retention times owing to interaction between the S-2-Butanol chiral modifier and chiral analytes was not observed.

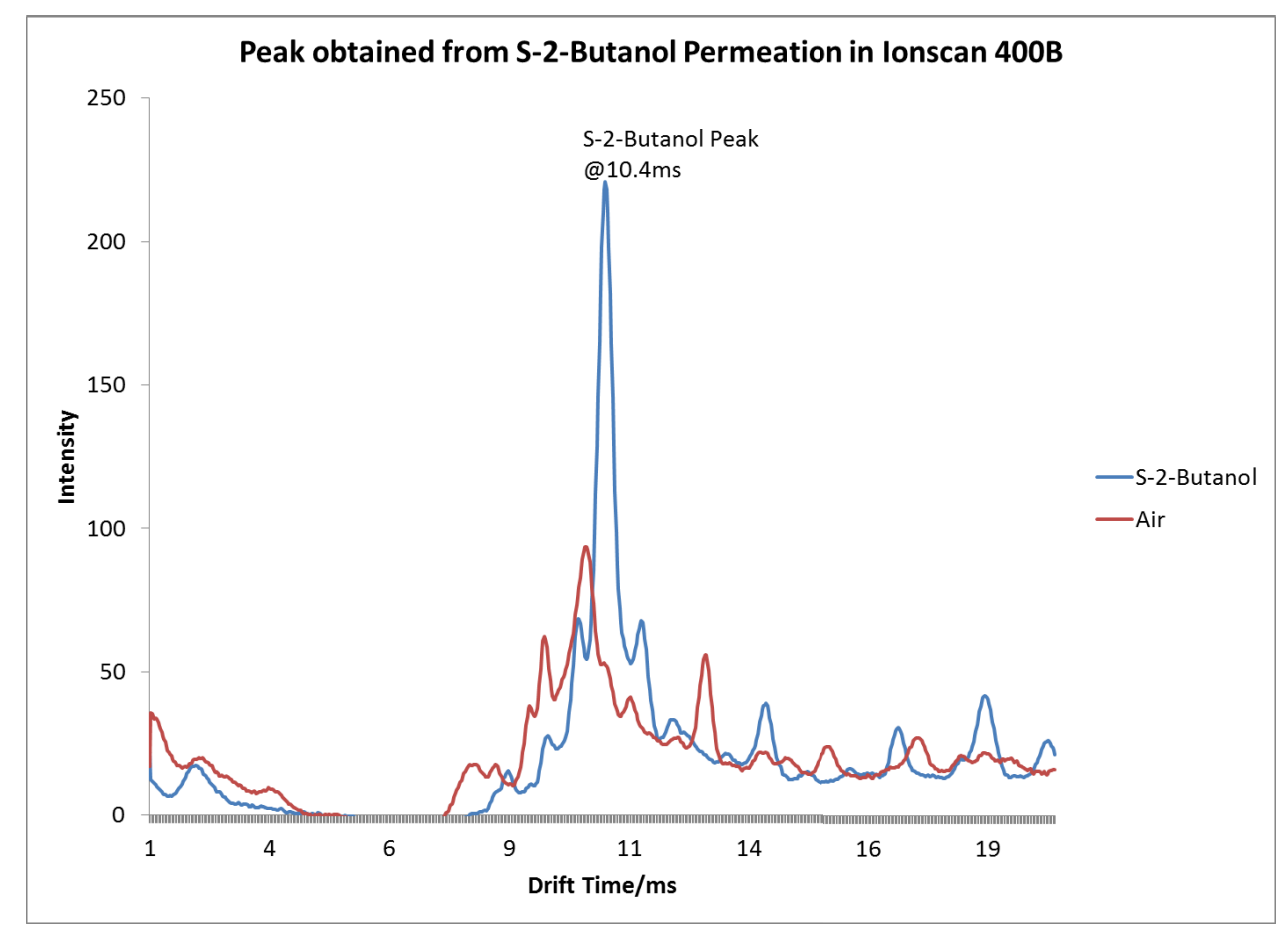

Figure 28: Peak obtained from S-2-Butanol permeation

The lack of interaction was believed to be attributed to a low ratio of chiral modifier to drift gas as the modifier was only permeating at a rate of $35 \mu \mathrm{L}$ per hour as opposed to the previously reported $45 \mu \mathrm{L}$ per hour [12], an alternative introduction system for the chiral modifier was investigated. The permeation rate could be increased through the use of additional heat, however, this could not be replicated precisely while conducting gravimetric analysis to determine the true permeation rate. Therefore, the application of 
heat was not utilized in order to increase the permeation rate and hence the ratio of modifier to drift gas. Forced induction through the use of an infusion pump was a possibility, as this allowed precise and accurate control of the ratio of modifier to drift gas. The infusion pump used was a Harvard apparatus model 22 syringe pump from Holliston, MA. The syringe pump was fitted with a $50 \mu \mathrm{L}$ Hamilton Gas tight syringe from the Hamilton Company, Reno, NV and set at various flow rates ranging from 50 to $400 \mu \mathrm{L} / \mathrm{Hr}$. In order to deliver the modifier to the drift gas, a direct connection could not be made as the drift tube and source of the Ionscan 400B were completely sealed by the manufacturer as a failsafe resulting from the presence of the radioactive ${ }^{63} \mathrm{Ni}$ source. Therefore, connections to infuse the modifier were done indirectly by using a Swagelok $1 / 8$ inch " $T$ " fitting connected between the positive dopant line and the dopant chamber to allow the introduction of the chiral modifier through a $0.50 \mu \mathrm{m}$ ID neutral capillary tube from Beckman Coulter, Brea, CA (Figure 29).

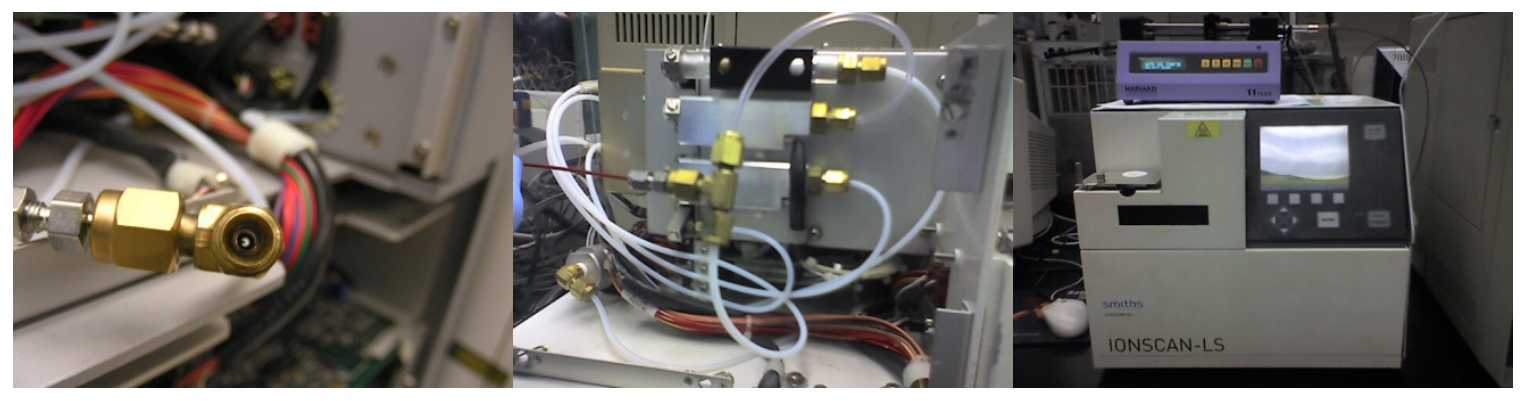

Figure 29: Images showing arrangement for direct infusion of modifier on Ionscan 400B

The arrangement provided much flexibility by allowing a range of infusion rates for the modifier to be introduced into the Ionscan 400B through varying modifier infusion rates. Experiments were carried out over a series of infusion rates using S-2-Butanol modifier, from $0 \mu \mathrm{L} / \mathrm{hr}$ to $450 \mu \mathrm{L} / \mathrm{hr}$. To ensure positive results, analytes previously reported by 
Dwivedi et al. were also used for these experiments. The stereoisomers $\mathrm{D}$ and $\mathrm{L}$ methionine were selected as these analytes had revealed the ability to be separated by gas phase chiral analysis [12]. Analysis of these compounds on the Ionscan 400B using air as the reactant peak reveals the analytes with a drift time of $11.75 \mathrm{~ms}$ each, diazepam which was also analyzed as a non-chiral standard reference compound showed a drift time of $16.12 \mathrm{~ms}$ (Figure 30).

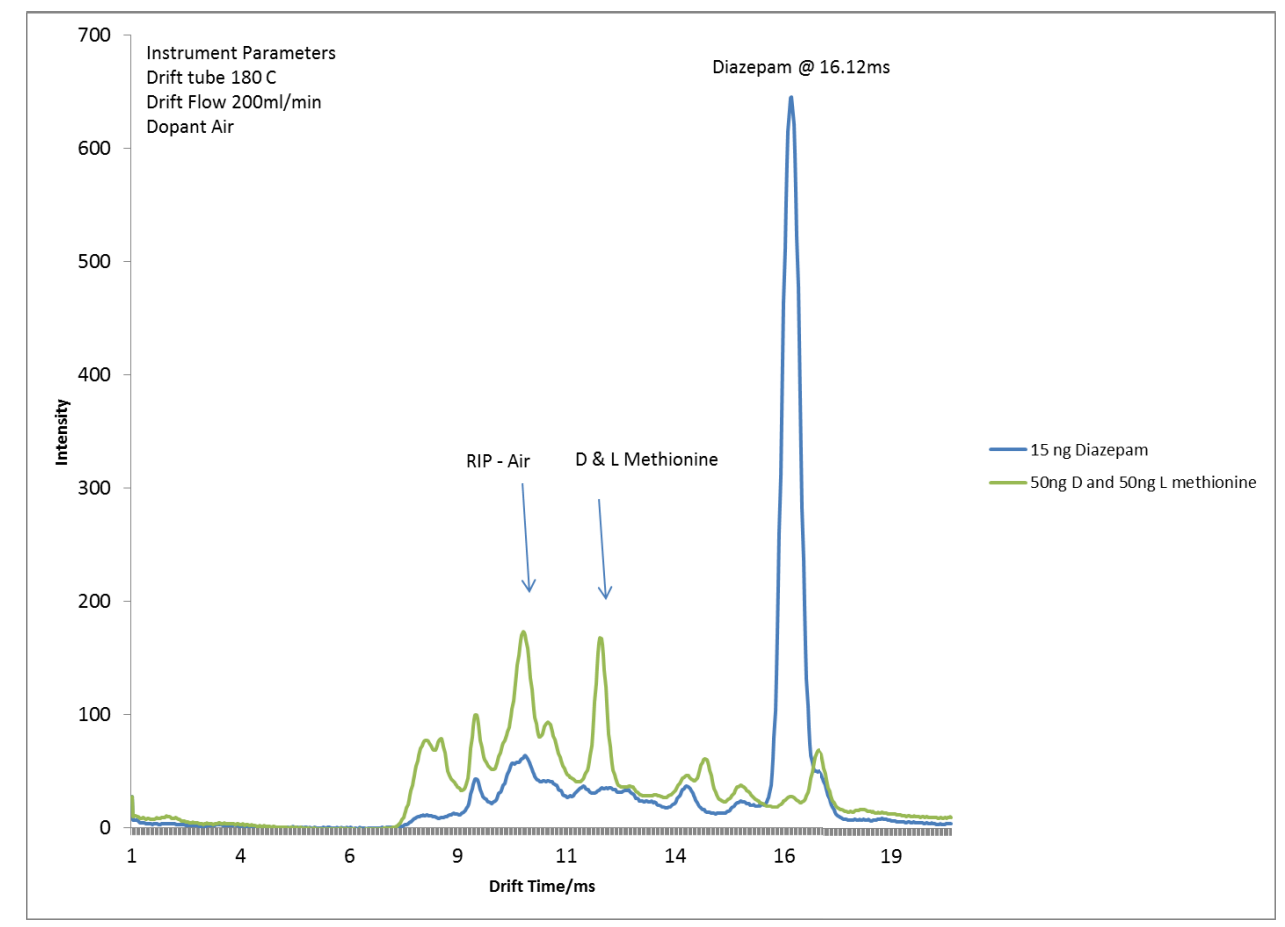

Figure 30: Ionscan 400B analysis of $D$ and $L$ methionine without modifier

The identical analytes run while infusing S-2-Butanol as the chiral modifier can be seen in Figure 31. The results of these experiments did not reveal much promise as the analytes D and L methionine showed no measurable separation. Of interest was the fact that the modifier also had an effect on the diazepam which had a shift in drift time from $16.12 \mathrm{~ms}$ to $16.55 \mathrm{~ms}$. The methionine which was a racemic mixture of both $\mathrm{D}$ and $\mathrm{L}$ 
enantiomers also had a shift in retention time from $11.75 \mathrm{~ms}$ to $14 \mathrm{~ms}$ (Figure 31 ). Other observations included the overall cleaner spectra observed when the modifier was infused resulting from the modifier suppressing the ionization of some unwanted species within the drift gas.

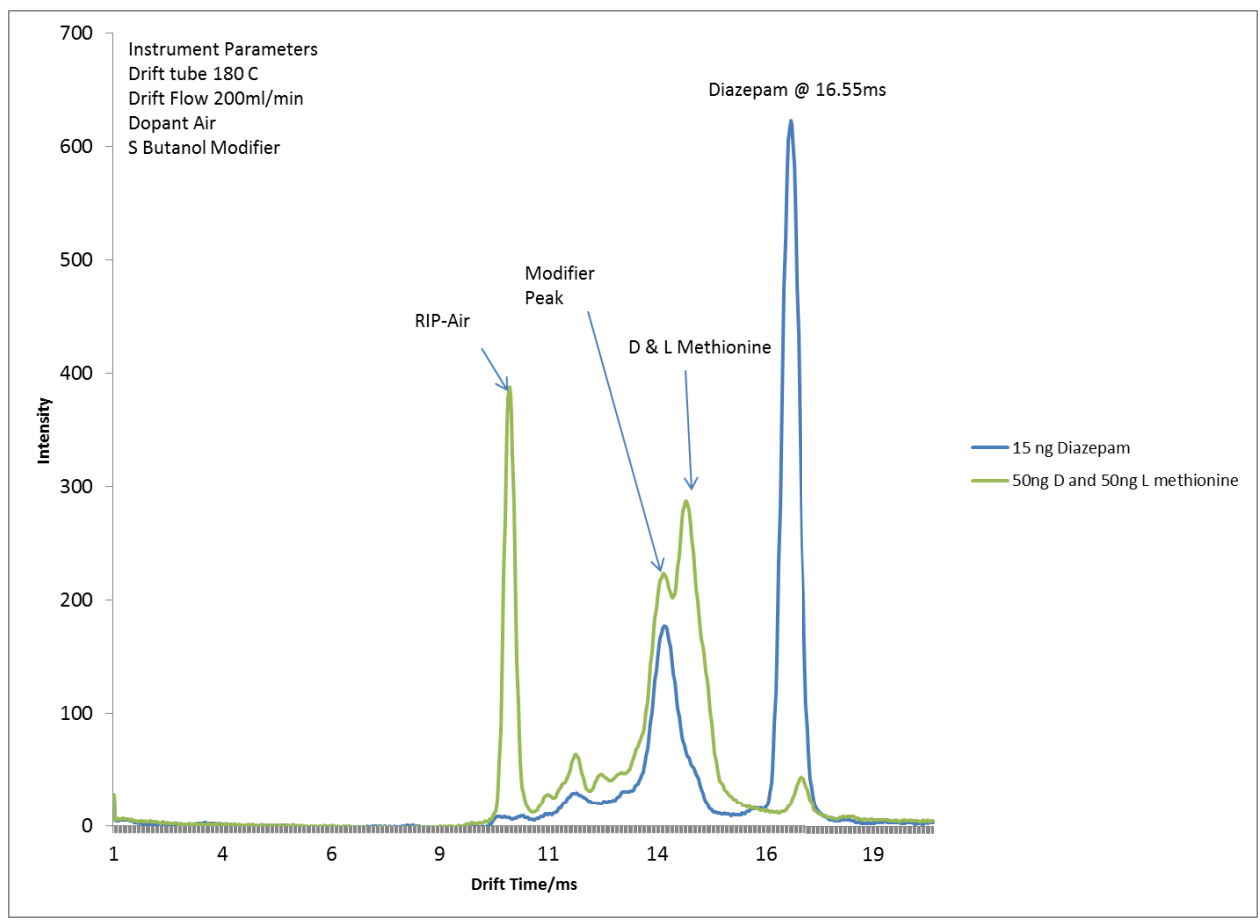

Figure 31: Ionscan 400B analysis of $D$ and $L$ methionine using S-2-Butanol modifier

On the basis of the significant change in drift time observed between the optically active methionines and the S-2-Butanol, a series of experiments were carried out in an attempt to improve upon this interaction and cause separation. During these experiments it was discovered that the modification made to the Ionscan 400B to introduce the modifier into the drift tube (Figure 29) had caused a large amount of the modifier to accumulate within the " $\mathrm{T}$ " fitting and so not be delivered to the drift tube. To improve mass transport of the modifier into the drift tube, the flow rate of the drift gas was increased from $200 \mathrm{ml} / \mathrm{min}$ to $300 \mathrm{ml} / \mathrm{min}$. Through a series of experiments at varying infusion rates from 0 to $400 \mu \mathrm{L}$ 
per hour, a set of parameters was determined that resulted in the separation of D and L methionine using S-2-butanol within the Ionscan 400B (Figure 32).

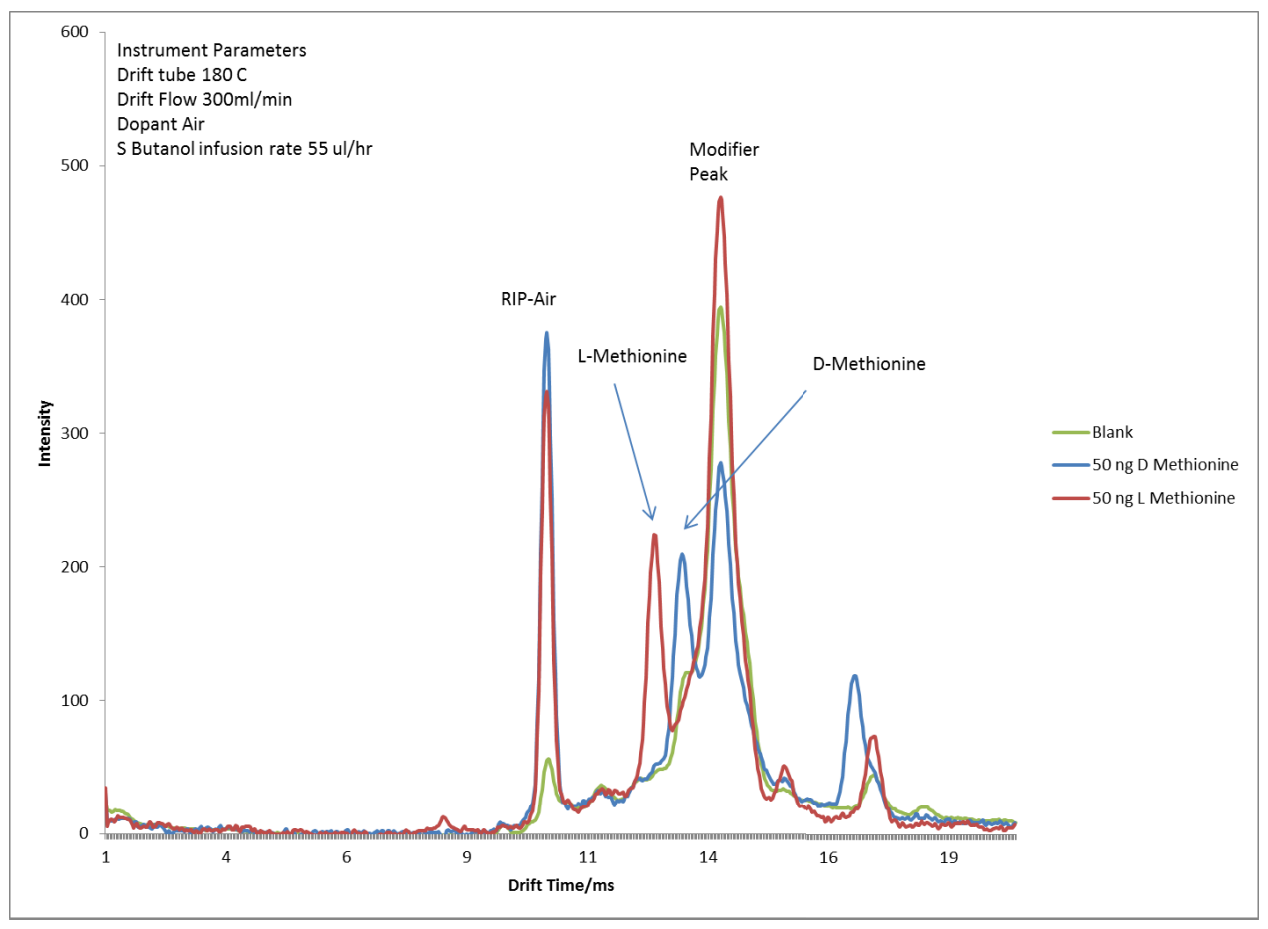

Figure 32: Improved chiral separation of $D$ and $L$ methionine on Ionscan 400B using S-2-Butanol modifier

The separation obtained not only verified the results reported by Dwivedi et al. [12], but also revealed that an OTS IMS could in fact be used to conduct chiral gas phase analysis albeit with some modifications. The next stage of the experiments was to apply these parameters to the target analytes of forensic interest which were the amphetamine substances (ATS). The Ionscan 400B proved quite suitable for the analysis of amphetamine type substances in the positive ionization mode. All target ATS enantiomers (R and S) methamphetamines, (RS and SR) ephedrines, (RR and SS) pseudoephedrines and ( $\mathrm{R}$ and $\mathrm{S}$ ) cathinones were detected resulting in ion mobility spectra with very sharp and intense peaks (Figure 33). 


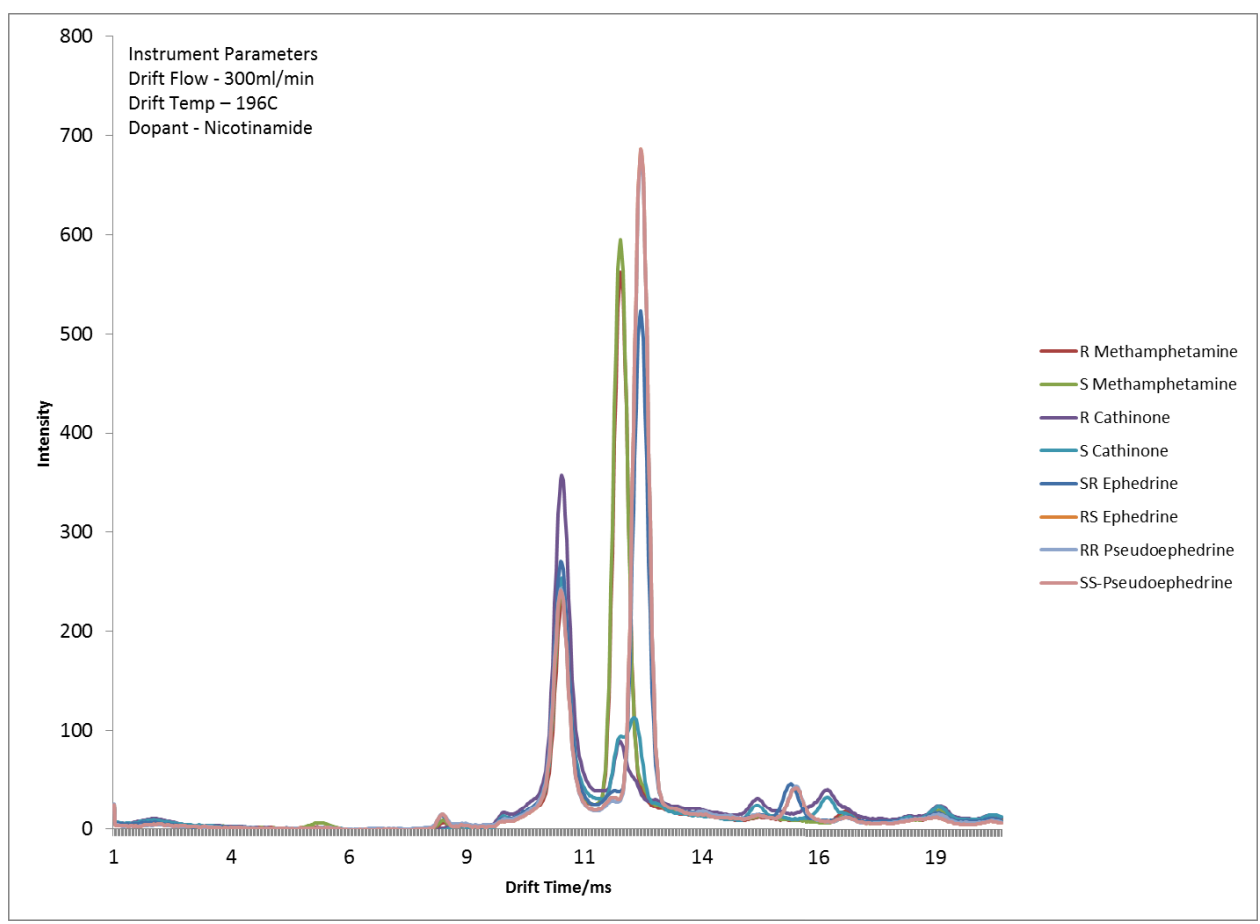

Figure 33: Plasmagram of Amphetamine Type Substances (ATS) analyzed by Ionscan400B

Of interest was the fact that these same analytes when analyzed on another OTS ion mobility unit, the Itemiser 2 manufactured and sold by General Electric (GE), not all analytes were detected as shown in Figure 34. The lack of detection was believed to be as a result of design differences between the two commercially available units. While Ionscan units have an open inlet that allows samples to be thermally desorbed directly into the ionization region (Figure 23), the GE Itemiser 2 utilizes a closed inlet by way of a semi-permeable membrane that helps eliminate unwanted contaminants from entering the ionization region and hence the drift tube. Though no further tests were carried out utilizing the Itemiser 2 to exhaustively investigate the cause for the lack of detection of key analytes such as ephedrine and pseudoephedrine, the membrane is thought to be impermeable to these analytes hence preventing them from entering into the ionization 
region of the Itemizer 2 and not being detected as shown in Figure 34. The Itemiser 2 has been upgraded and is now available as the Itemiser 3 sold by Safran Morpho that acquired GE security in 2009 [106]. A cautionary note, the technology of utilizing a semipermeable membrane is still the same with current Morpho IMS units as with the previous Itemiser 2 and hence the same inability to detect various compounds may still exist even in these newly updated OTS devices sold by Safran Morpho.

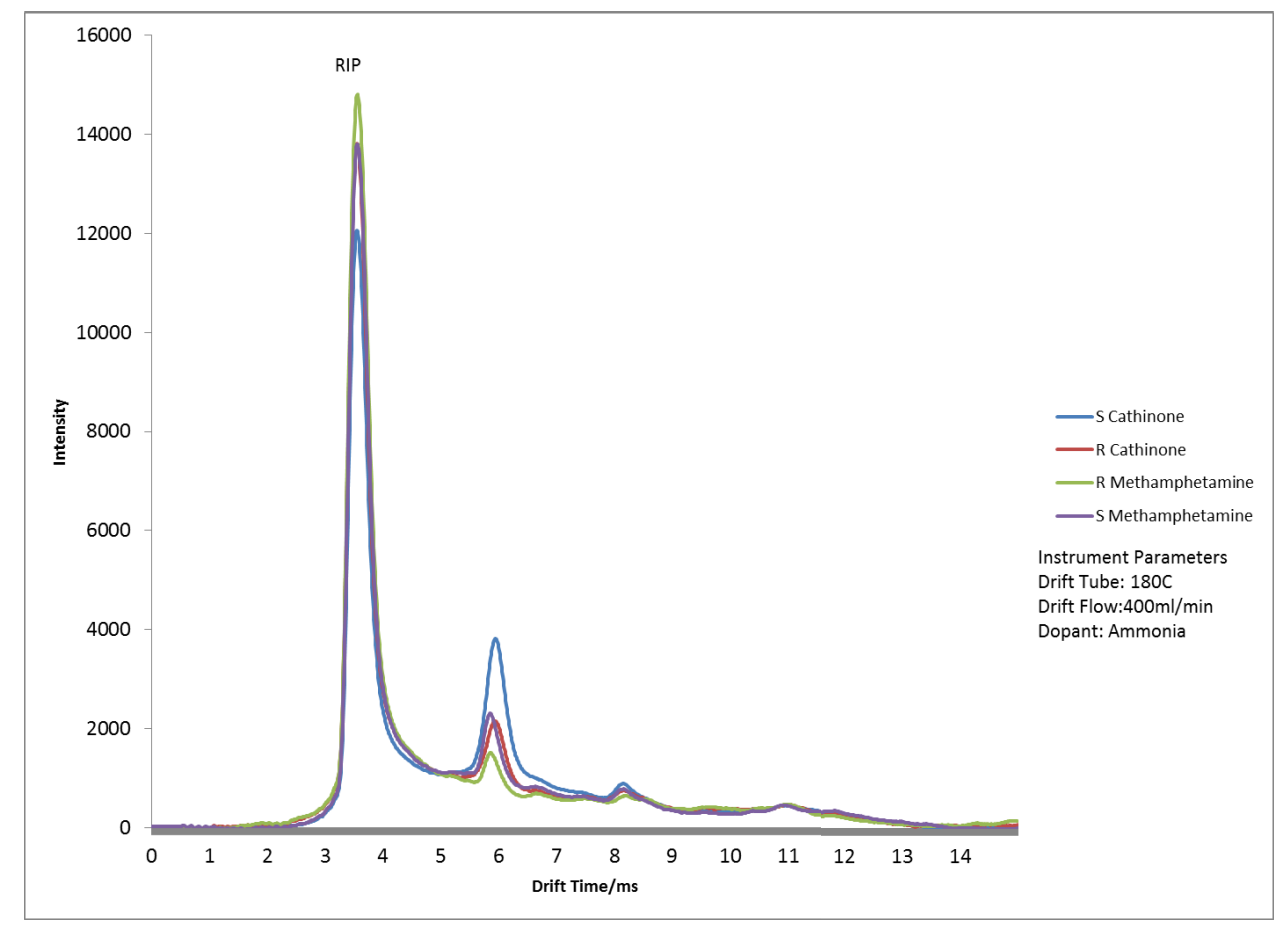

Figure 34: Plasmagrams of ATS analyzed by GE Itemiser 2 showing only the detection of Methamphetamine and cathinone, ephedrine and pseudoephedrine cannot be detected.

Figure 33 of ATS analyzed by the Ionscan 400B does show very sharp peaks, but it also shows that there is much overlap between the analytes as indicated by their drift times (Table 1). 
Table 1: Drift Times of ATS analyzed on Ionscan 400B

\begin{tabular}{|l|r|}
\hline \multicolumn{2}{|l|}{ Drift times of Analytes analyzed on Ionscan 400B } \\
\hline Analyte & Drift Time/ms \\
\hline R-Methamphetamine & 11.784 \\
\hline S-Methamphetamine & 11.777 \\
\hline RS-Ephedrine & 12.201 \\
\hline SR-Ephedrine & 12.201 \\
\hline RR-Pseudoephedrine & 12.21 \\
\hline SS-Pseudoephedrine & 12.208 \\
\hline R-Cathinone & 11.781 \\
\hline S-Cathinone & 11.778 \\
\hline
\end{tabular}

The purpose of modifying the Ionscan 400B would allow for the introduction of the S-2Butanol chiral modifier which should eliminate this overlap by providing separation between these different analytes. The expected result being similar to that obtained from the separation of D and L methionine (Figure 32), however, numerous challenges were faced with the attempted chiral separation of ATS utilizing the Ionscan 400B. All ATS introduced into the Ionscan 400B did show a significant change with their drift time when the S-2-butanol chiral modifier was also introduced. The shift in drift times was found to be proportional to the relative ratio of modifier to drift gas, that is, the greater the amount of modifier introduced, the greater the shift in the drift time. Four sets of enantiomers were targeted of the eight ATS studied as these provided the greatest forensic impact. These were RS and SR ephedrine and RR and SS pseudoephedrine. To enhance separation capabilities even further, parameters that could be changed with the Ionscan 400B that were configurable by the manufacturer were also investigated. The parameter within ion mobility that has the single greatest effect on the instrument's resolving power is the width of the gate pulse. To demonstrate the effect of gate pule on resolving power, two analytes with relatively close reduced mobilities were analyzed on the Ionscan 400B, 
diazepam $\left(\mathrm{K}_{\mathrm{o}} 1.211 \mathrm{~cm}^{2} \mathrm{~V}^{-1} \mathrm{~s}^{-1}\right)$ and Cocaine $\left(\mathrm{K}_{\mathrm{o}} 1.160 \mathrm{~cm}^{2} \mathrm{~V}^{-1} \mathrm{~s}^{-1}\right)$. Figure 35 shows the ion mobility spectra of diazepam and cocaine analyzed with the default gate pulse of the Ionscan 400B of 200 microseconds $(0.2 \mathrm{~ms})$. At this gate pulse, the analytes are resolved with a resolution of 0.43 and a resolving power of 36 for each analyte.

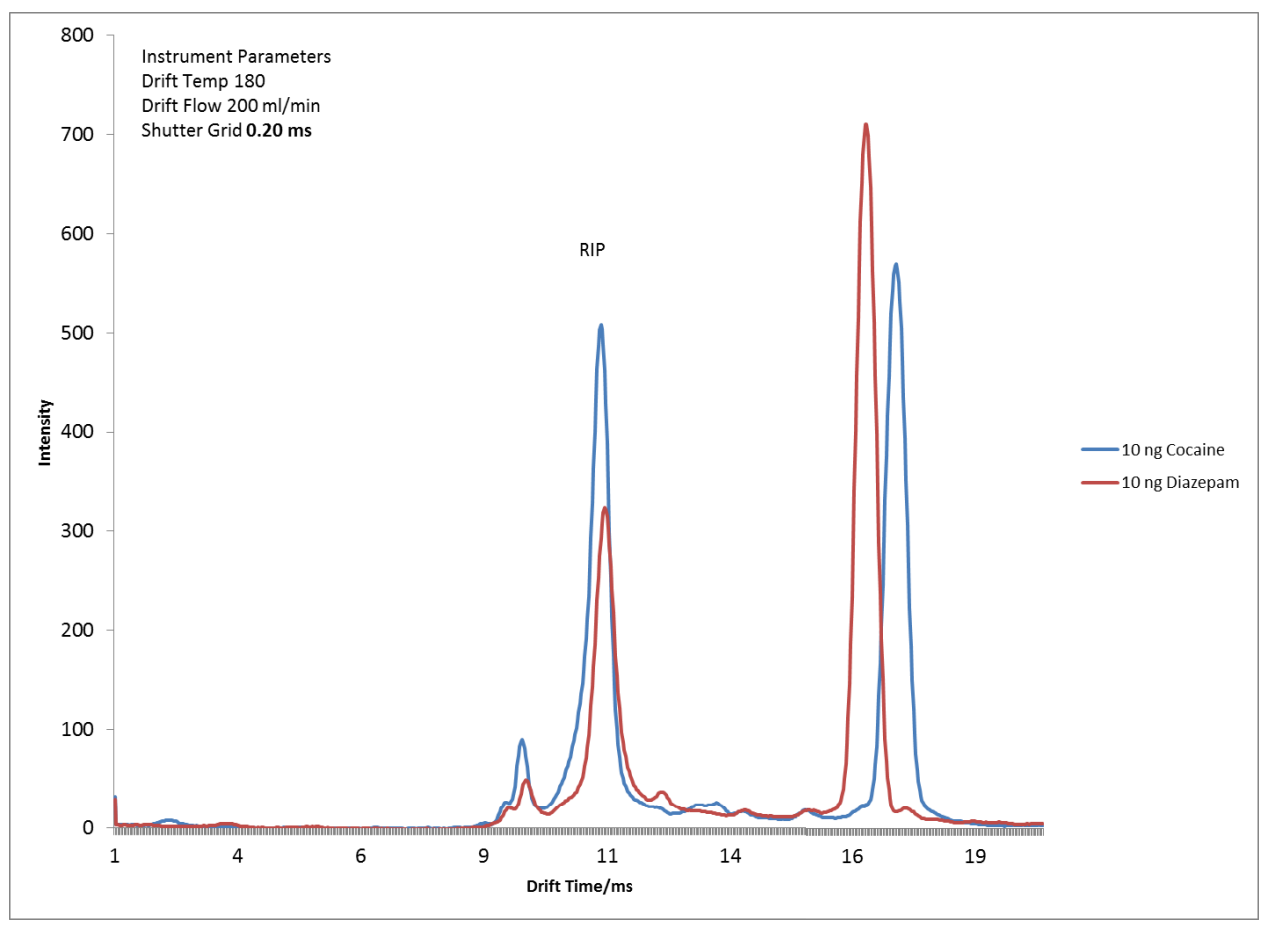

Figure 35: Diazepam and Cocaine analyzed on Ionscan 400B with $0.2 \mathrm{~ms}$ Gate Pulse

By reducing the gate pulse, these values can be improved. The gate pulse was therefore reduced to a value of $50 \mathrm{microseconds}(0.05 \mathrm{~ms})$ within the $400 \mathrm{~B}$ to provide more resolved spectra as shown in Figure 36. 


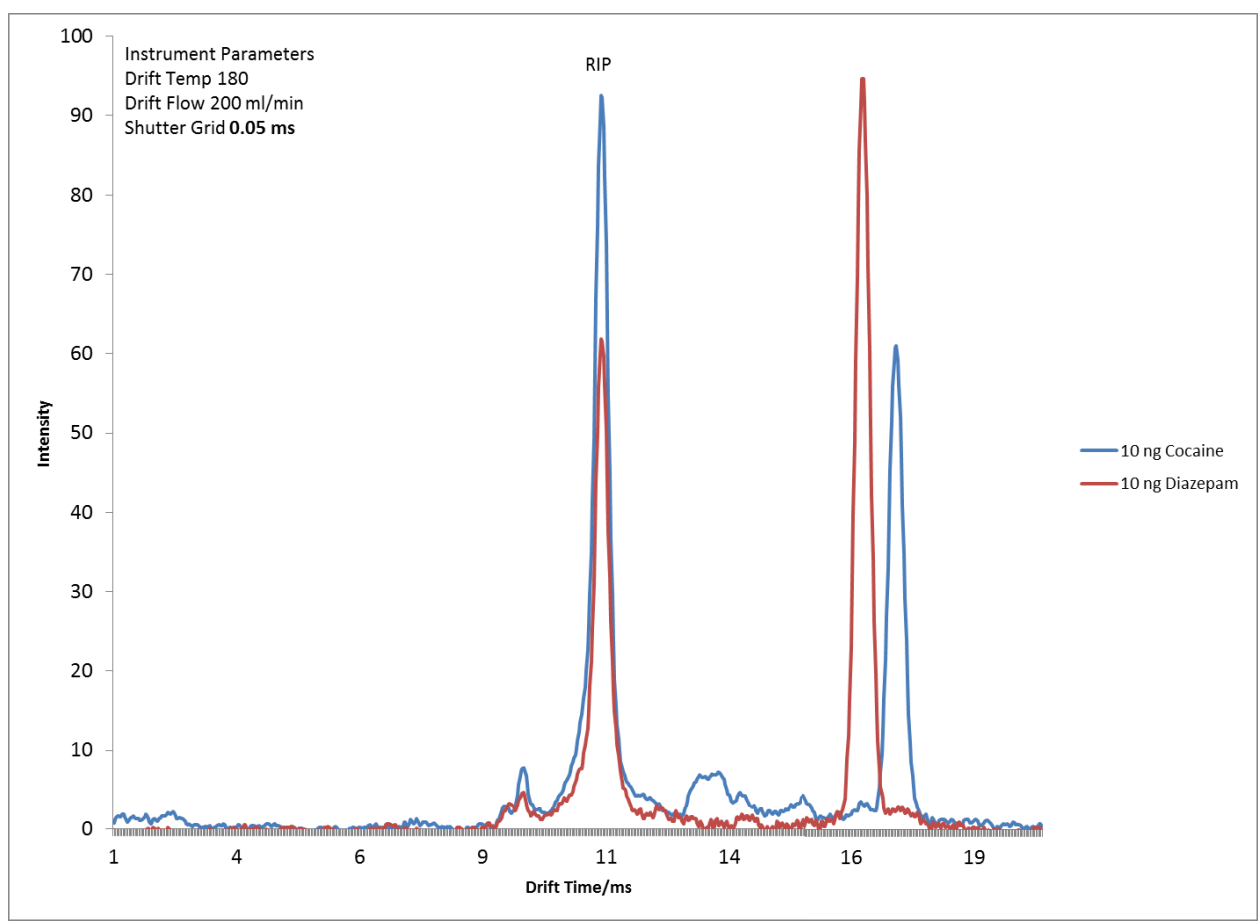

Figure 36: Diazepam and Cocaine analyzed on Ionscan 400B with 0.05ms Gate Pulse showing improved resolving power

The reduced gate pulse increased the resolution between the two peaks from 0.43 at the default gate pulse, to 0.65 and the resolving power of each peak increased to 40 . The overall trend can be seen in Figure 37. However, there is a caveat when reducing the gate pulse that can be seen when comparing the signal intensities of Figure 35 and Figure 36. Reducing the gate pulse does in fact improve resolution but it does so at the expense of decreasing signal intensity. 


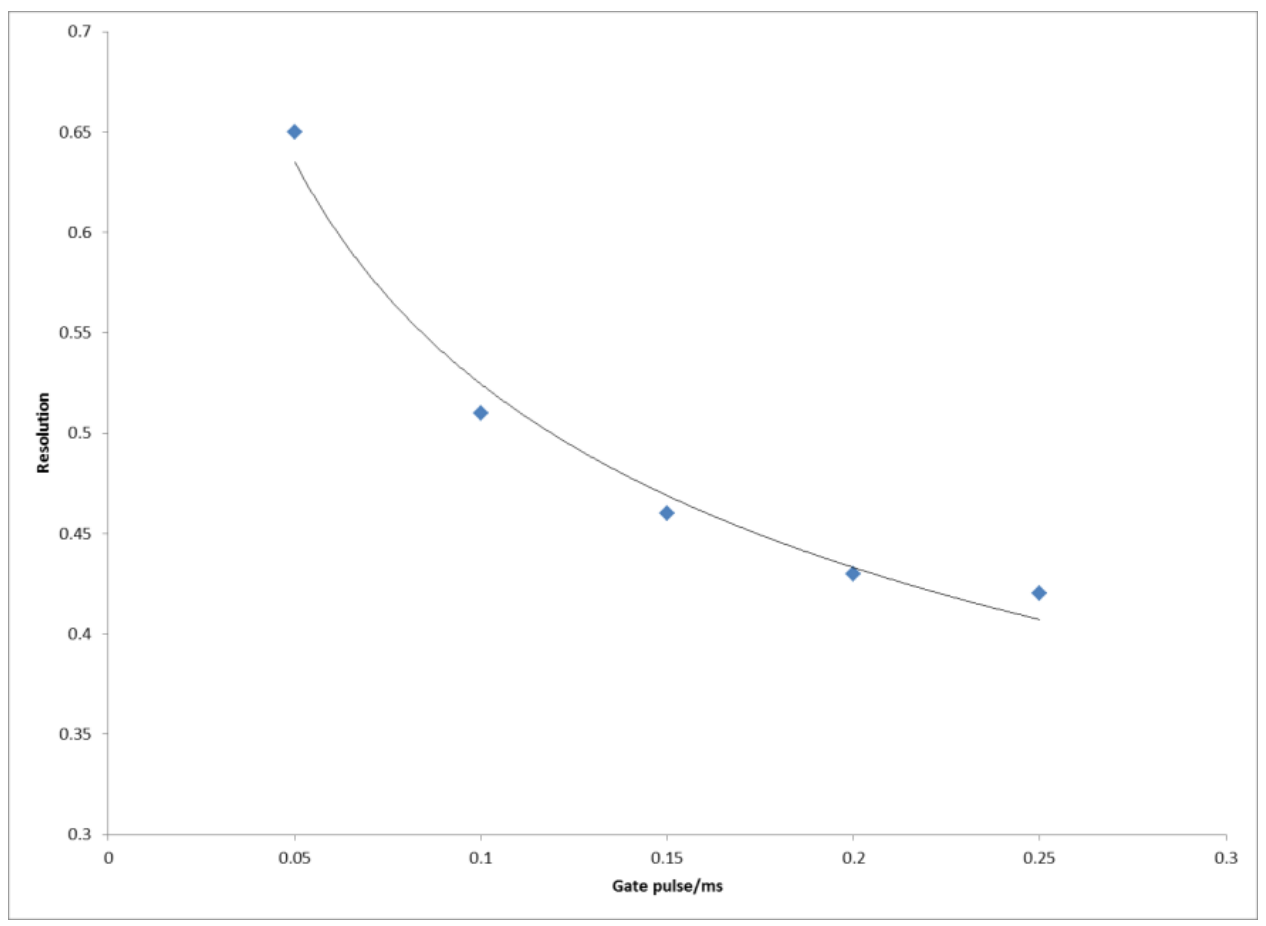

Figure 37: Effect of Gate pulse on resolution between Diazepam and Cocaine

As the gate pulse is shortened, the ion swarm allowed to enter the drift tube is confined into tightly packed or thinly sliced bands. The thin band experiences less diffusion or band broadening as it migrates down the drift tube, resulting in peaks with higher resolving power and hence greater resolution. But the shorter and more thinly sliced ion swarm is also smaller resulting in much less ions being detected overall as shown in Figure 38. 


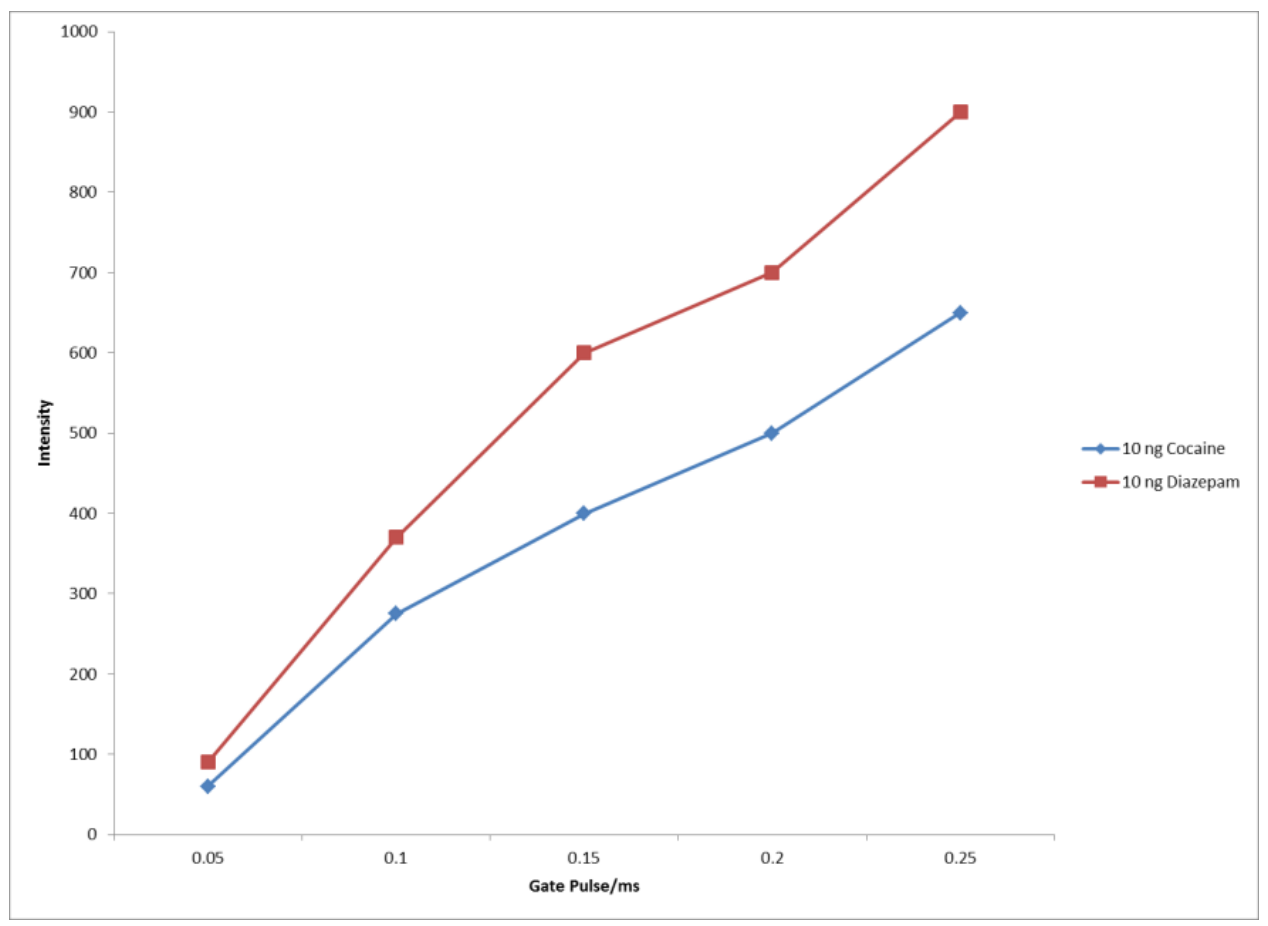

Figure 38: Ion intensity vs. Gate Pulse duration on Ionscan 400B

The smaller number of ions detected was quite significant on the Ionscan 400B as Figure 38 shows an approximate $90 \%$ decrease in signal intensity for both diazepam and cocaine when changing the gate pulse from $0.2 \mathrm{~ms}$ to $0.05 \mathrm{~ms}$ while providing only a $10 \%$ increase in resolving power. Interestingly enough, Figure 37 does imply that the resolution obtained between these two analytes could be infinitely large with smaller and smaller gate pulses, but the instrument would most certainly be unable to detect these analytes at that point. In fact at $0.05 \mathrm{~ms}$ gate pulse, the Ionscan $400 \mathrm{~B}$ was unable to perform any data analysis functions on the obtained peaks shown in Figure 36 as a result of the low signal intensity. Though the theory behind improving the overall resolution of ion mobility through the manipulation of the gate pulse is sound, the application on the Ionscan 400B did not provide exceptional results, therefore the default $0.2 \mathrm{~ms}$ gate pulse was adhered to for remaining experiments. Table 1 shows the obtained drift times of ATS analyzed on 
the Ionscan 400B. By focusing only on those analytes with the highest forensic interest, RS and SR ephedrine and RR and SS pseudoephedrine a clearer picture of the Ionscan's potential could be determined.

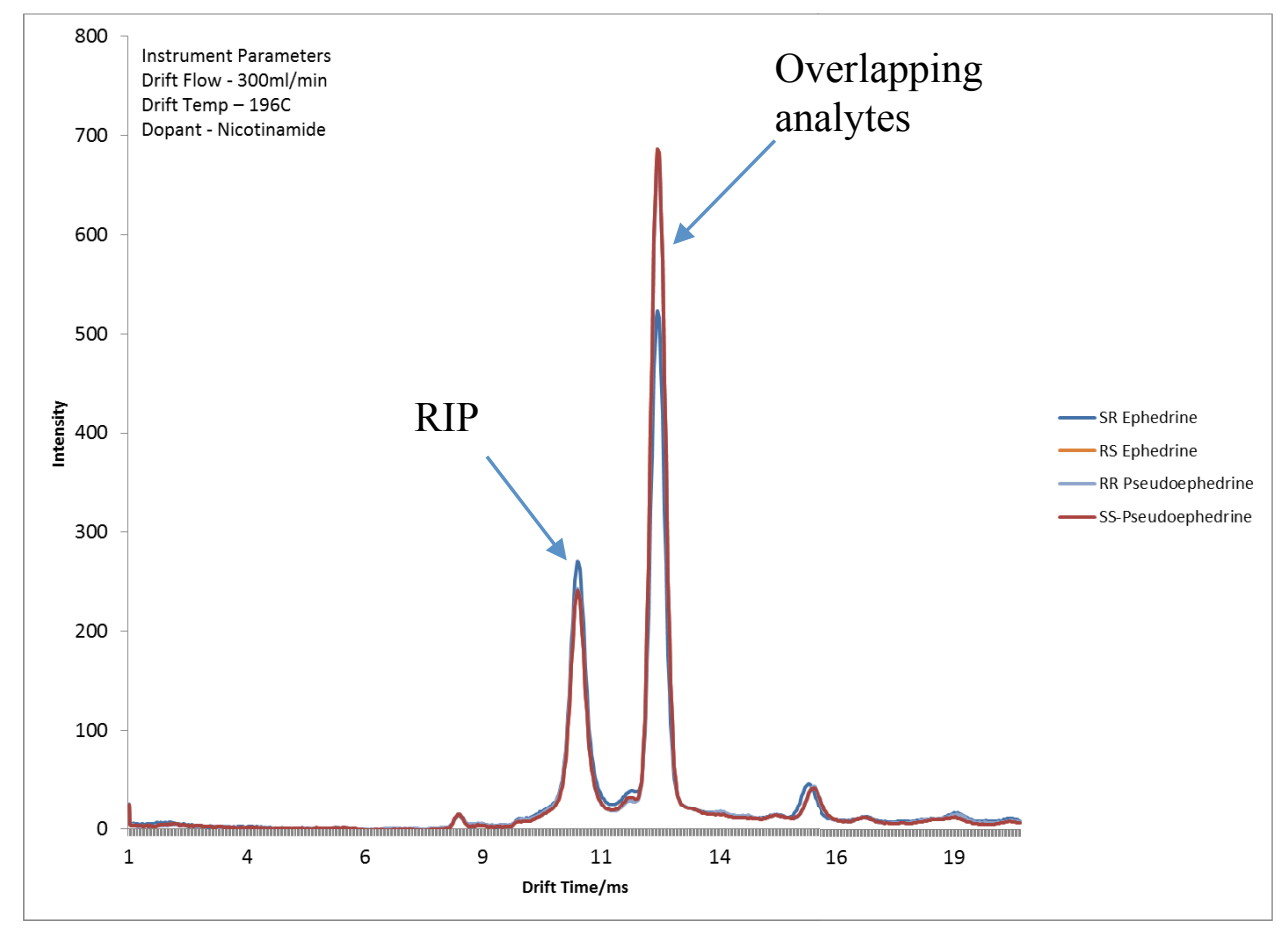

Figure 39: Separation of select ATS on Ionscan 400B

Figure 39 shows that there is near perfect overlap of these chiral molecules when conducting traditional ion mobility experiments on the Ionscan 400B. By introduction of the S-2-butanol chiral modifier, these analytes begin to separate in a manner that is proportional to the relative amount of modifier being introduced into the system. The introduction of the modifier as mentioned previously, was shifted from a permeation system Figure 27 to a positive infusion system that allowed for more precise control and faster delivery of the modifier Figure 29. 


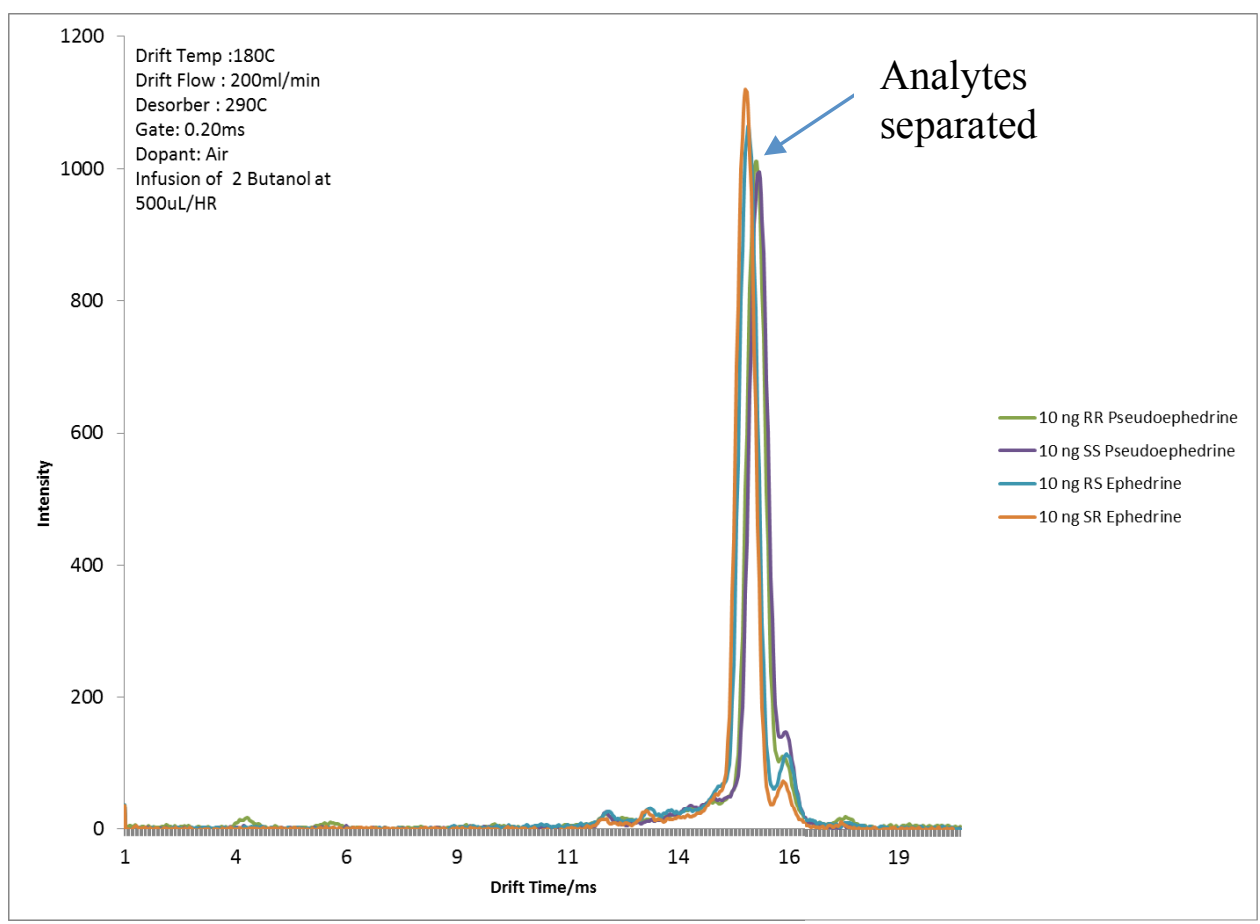

Figure 40: Chiral separation of select ATS using S-2-Butanol at 500uL per hour infusion

Figure 40 shows that these chiral molecules are able to be separated to some extent by using the chiral S-2-Butanol modifier. (RS and SR) ephedrine are separated from (RR and SS) pseudoephedrine which were previously unresolved as shown in Figure 39. Relatively high infusion rates of $500 \mu \mathrm{L}$ per hour were required in order to achieve this separation providing resolution of only 0.15 between these previously unresolved analytes. Of interest, was the observation that as the analytes' drift times were retarded by the S-2-Butanol modifier, the analytes drifted closer to the modifier, resulting in some overlap of the modifier and analyte thereby reducing the overall resolution of the technique. Coupled with this overlap between analyte and modifier was the realization that excessively high flow rates infused above $300 \mu \mathrm{L}$ per hour appeared to not have good mass transport from the brass "T" fitting shown in Figure 29, into the drift tube. Evident by the large amounts of condensed modifier found within the transfer lines after 
experiments at these flow rates and also from the overall separation plotted of the drift time separation of these analytes versus the infusion rate of the modifier Figure 41.

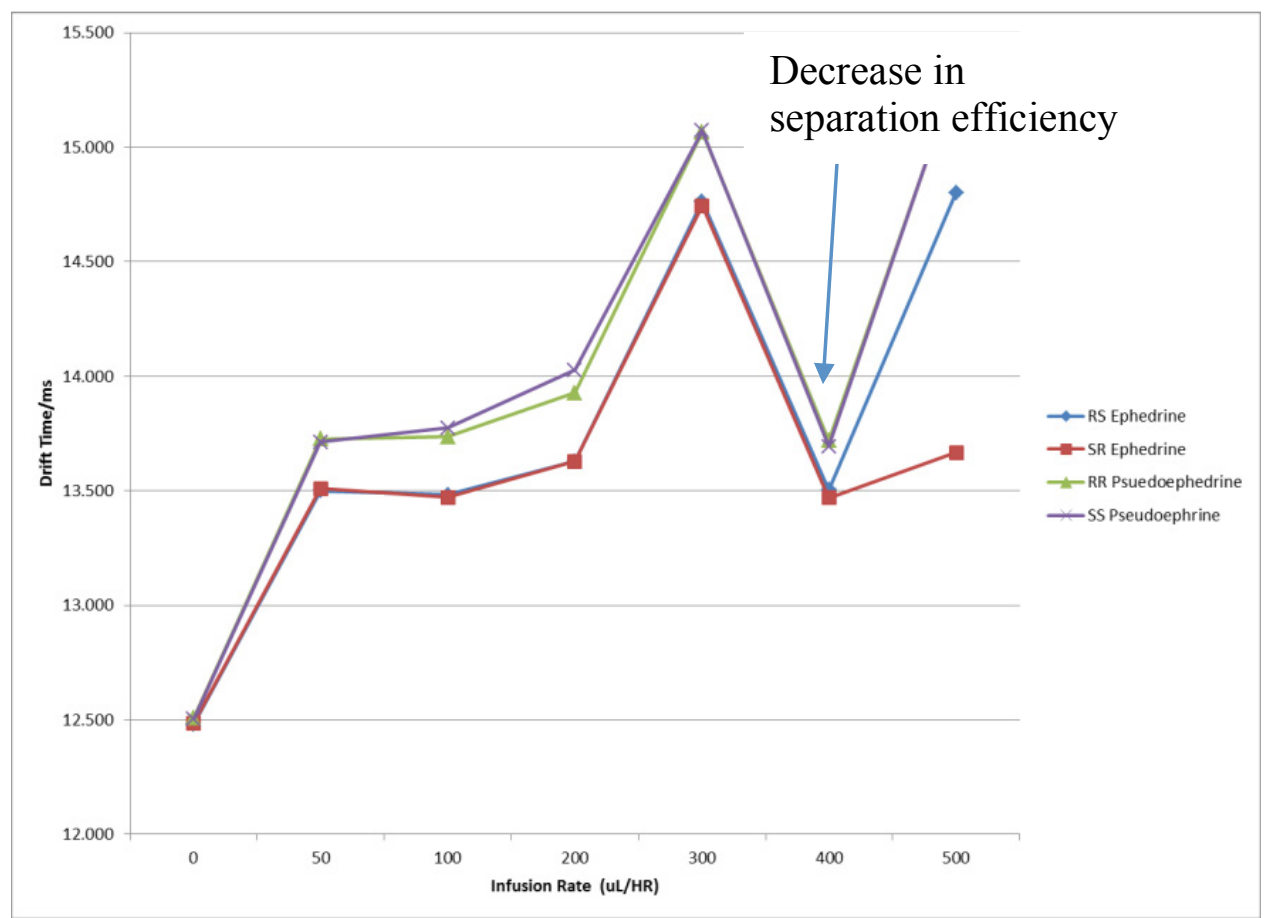

Figure 41: Effect of Modifier infusion rate on drift time separation of analytes on Ionscan 400B

Therefore on subsequent experiments, flow rates were maintained between 0 and $300 \mu \mathrm{L}$ per hour which resulted in much less accumulation of condensed modifier within the transfer lines and hence better mass transfer of modifier into the drift tube. Throughout the course of these experiments it was found that both Cocaine and Diazepam were affected only marginally by use of the S-2-Butanol chiral modifier (Table 2). 
Table 2: Effect of Modifier on Drift time of Analytes

\begin{tabular}{|c|c|c|c|c|c|c|}
\hline \multirow[b]{2}{*}{$\begin{array}{c}\text { Modifier Flow } \\
\text { Rate ul/HR }\end{array}$} & \multicolumn{6}{|c|}{ Drift Time/ms } \\
\hline & RS Ephedrine & SR Ephedrine & RR Psuedoephedrine & SS Pseudoephrine & Cocaine & Diazepam \\
\hline 0 & 12.489 & 12.491 & 12.501 & 12.498 & 16.849 & 16.179 \\
\hline 50 & 12.869 & 12.731 & 12.81 & 12.79 & 16.812 & 16.287 \\
\hline 100 & 12.945 & 12.977 & 13.061 & 13.078 & 16.835 & 16.518 \\
\hline 200 & 13.391 & 13.378 & 13.512 & 13.576 & 16.862 & 16.673 \\
\hline 300 & 13.454 & 13.403 & 13.536 & 13.636 & 16.871 & 16.812 \\
\hline
\end{tabular}

Chiral analytes such as ephedrine and pseudoephedrine showed a relatively large change in drift times of approximately $1 \mathrm{~ms}$ when comparing no modifier infused to $300 \mu \mathrm{L}$ of modifier infused. This is represented in Table 2 and also graphically in Figure 42.

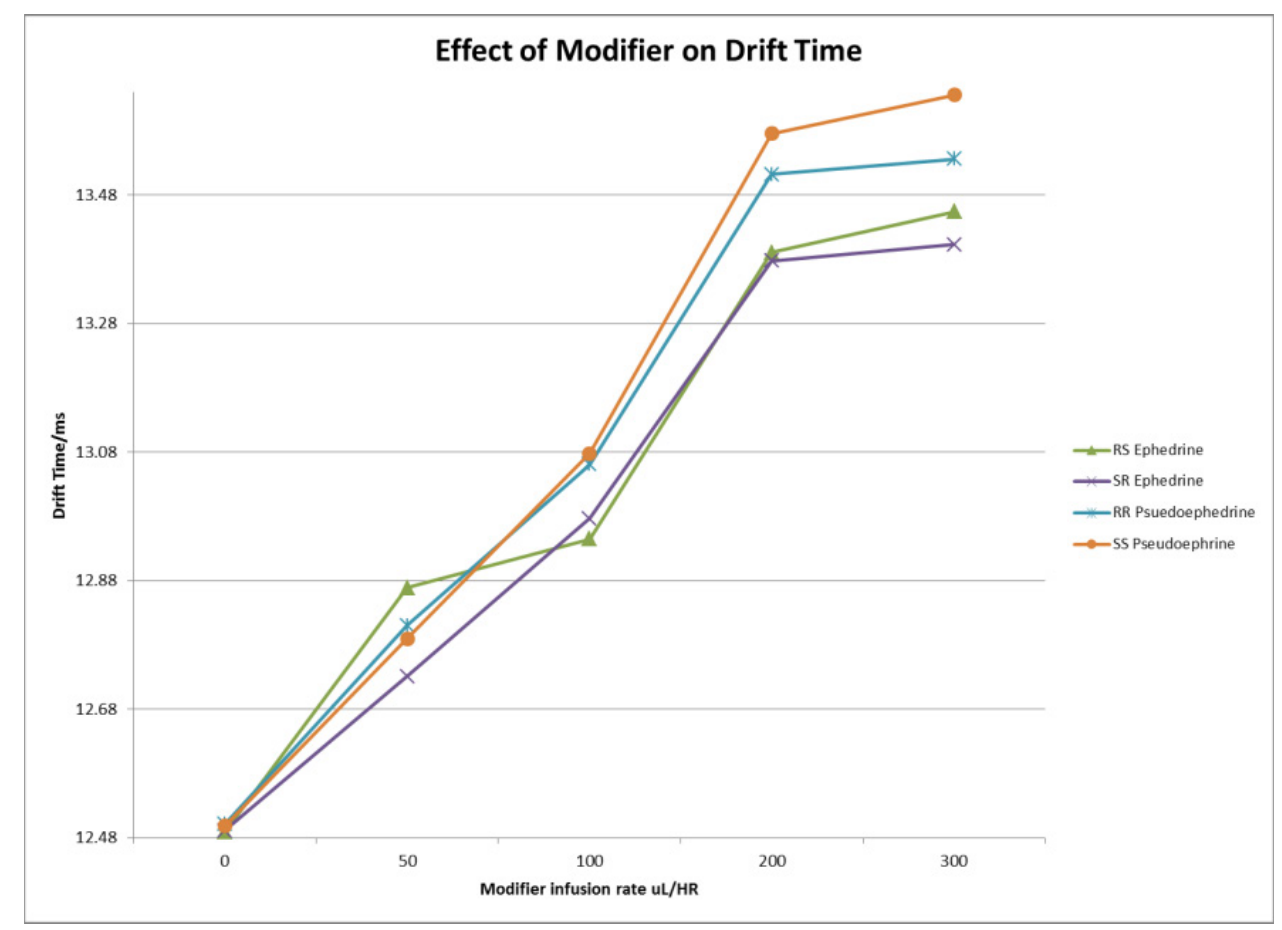

Figure 42: Effect of Modifier on drift time of Chiral Analytes

Diazepam however, showed a much smaller change of $0.6 \mathrm{~ms}$ over this same modifier infusion range while Cocaine showed essentially no change in drift time over this modifier infusion range as displayed graphically in Figure 43. 


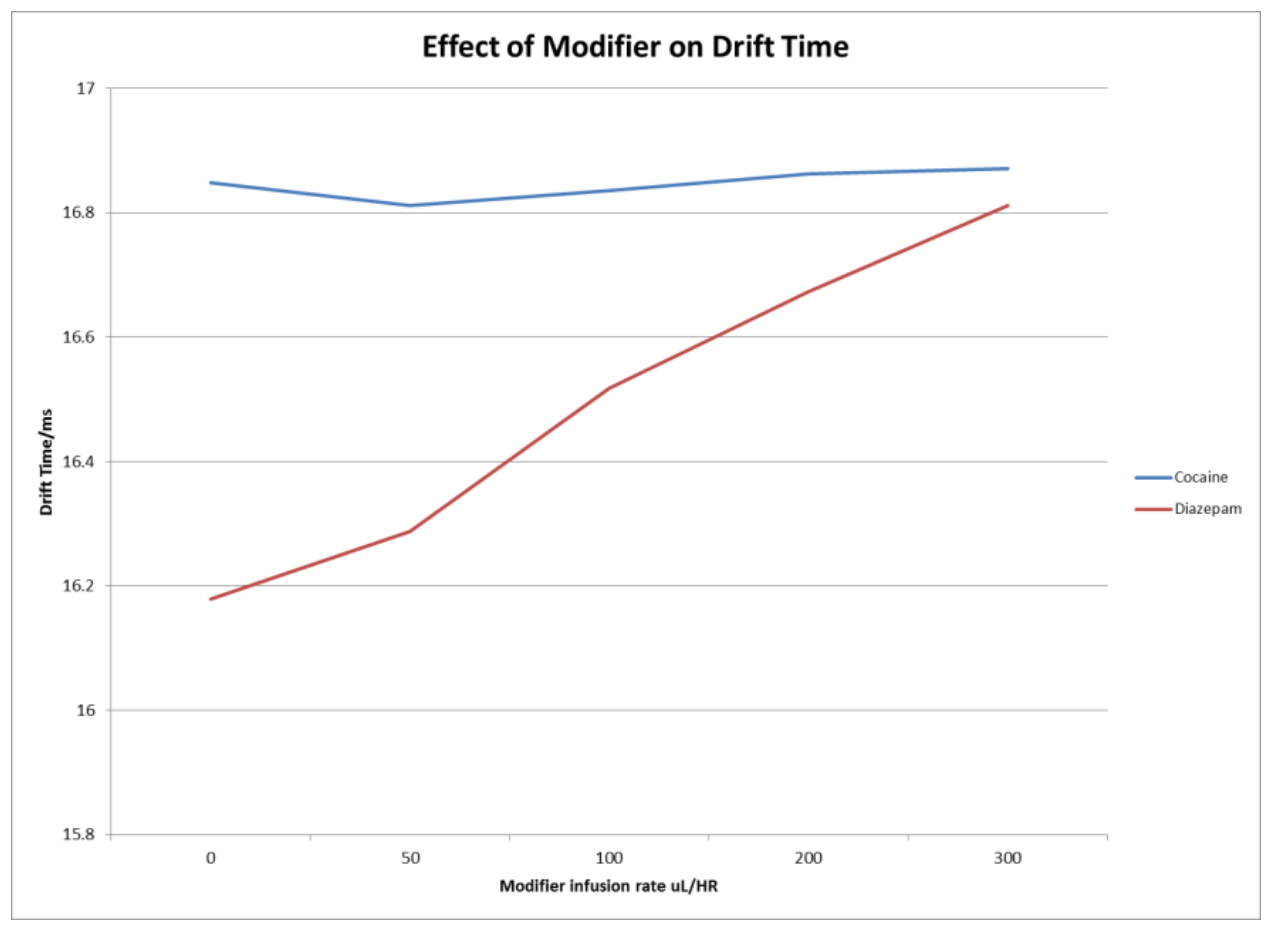

Figure 43: Effect of modifier on drift time of Cocaine and Diazepam

\subsubsection{Conclusions:}

The conclusions being drawn from these experiments therefore are as follows. It is possible to utilize an off the shelf IMS unit to conduct chiral separations provided that modifications are mode to ensure the efficient delivery of the modifier Figure 29. The modifier S-2-Butanol is effective in separating D and L Methionine (Figure 32) as well as amphetamine type substances (SR and RS) ephedrine from (RR and SS) pseudoephedrine shown in Figure 40. However, modifier introduction is required at relatively high infusion rates to cause separation, but with the changes made to the OTS Ionscan 400B, the infusion rates had to be maintained below $300 \mu \mathrm{L}$ per hour to prevent condensation and accumulation of modifier within transfer lines. It was also observed and concluded that the S-2-Butanol chiral modifier had negligible effect on the separation of other 
analytes namely Cocaine and Diazepam. On the basis of these conclusions the next phase of experiments would require a more efficient mechanism of modifier introduction into the IMS drift tube, an instrument with higher resolving capabilities to improve the overall separation between analytes and mas spectrometric capabilities in order to determine the type of species being formed within the drift tube during introduction of the chiral modifier. 


\subsection{Utilization of an Electrospray Ionization Ion Mobility Spectrometer for Chiral Separations}

The previous section highlighted the possibility and limitations of utilizing an OTS Ion mobility unit, the Ionscan 400B for the task of conducting chiral separations through the introduction of a chiral modifier. This current section will highlight the capabilities and results of utilizing a custom built Chiral Ion Mobility Unit for this same task. The unit being described is one of the only commercially available Ion mobility units that utilize an electrospray ionization (ESI) source. The source was more thoroughly described in section Theory of Electrospray Ionization (pg. 18). The unit was designed and built by Excellims corporation in Acton Massachusetts and comprises a high resolution liner drift tube Ion mobility spectrometer unit with a cool electrospray ionization source all coupled to a quadrupole mass spectrometer from Extrel corporation Figure 44 .

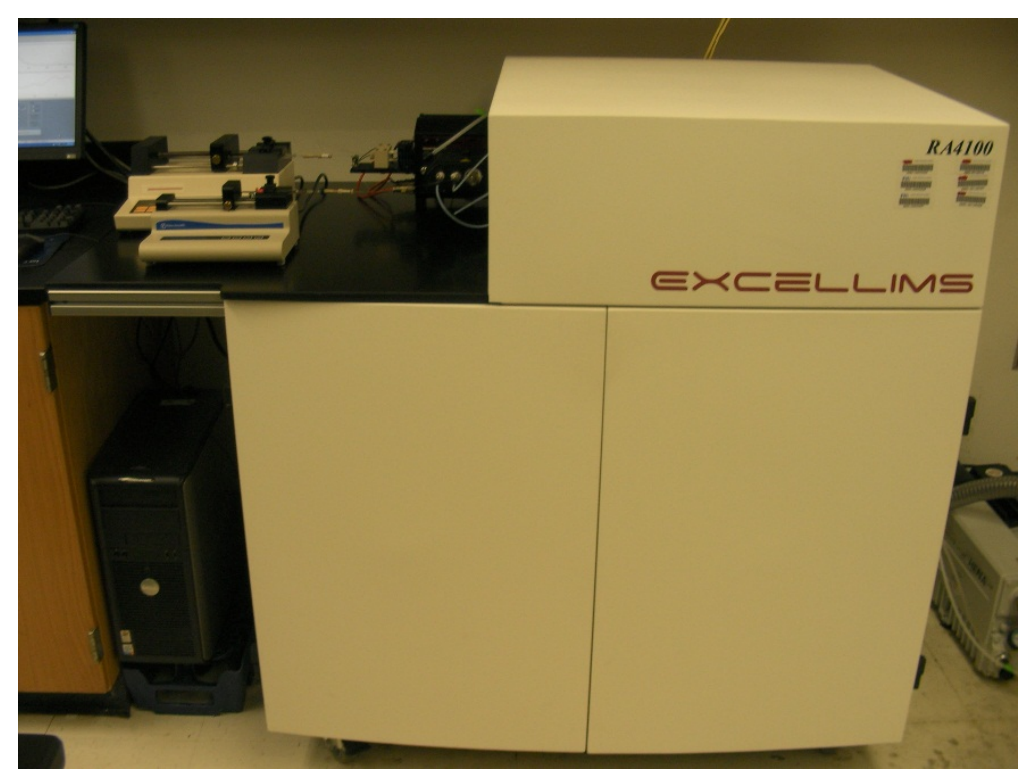

Figure 44: ESI-IMS-MS (RA4100)

As previously discussed, one of the benefits of a non-radioactive source such as ESI eliminated many of the environmental health and safety burdens which accompanies the 
traditional radioactive sources. The ability of the electrospray source to ionize samples while in solution allows for greater ionization of thermally labile species, as opposed to first being heated and vaporized into the gas phase with ${ }^{63} \mathrm{Ni}$ sources. The cool electrospray ionization source was developed by Dr. Ching Wu (CEO of Excellims) while under the tutelage of Dr. Herbert Hill [74].

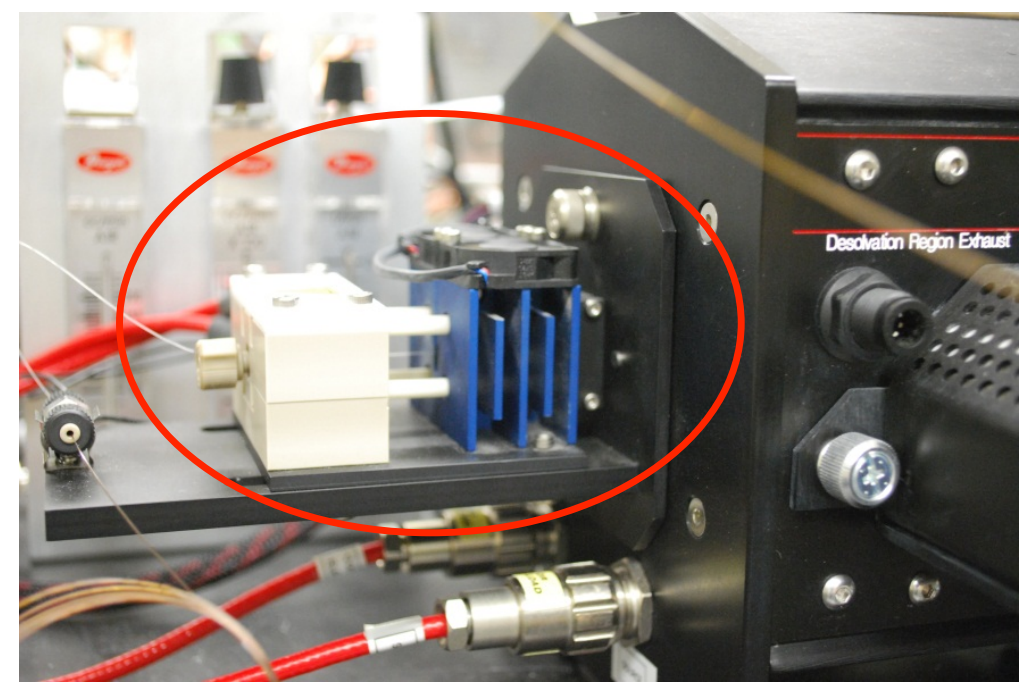

Figure 45: Cooled Electrospray Ionization Source on RA4100

The cooled electrospray ionization source on the RA4100 is air cooled vs. the water cooled unit that was originally used by $\mathrm{Wu}$ et al. [74]. The requirement of a cooled electrospray ionization unit stems from the fact that solvent would prematurely evaporate if a heated desolvation gas was used causing fouling of the ESI needle. However, there would be insufficient solvent evaporation and excessive peak broadening if a desolvation gas was not used [73]. The solution was a cooled ESI source which allowed the use of a desolvation gas at a mild temperature providing the benefit of desolvation without premature evaporation that fouls the ESI tip. The RA4100 also possesses a high resolution drift tube, producing resolving powers of 80 for some favorable analytes. The 
high resolving power is achieved through the RA4100's high voltage power module Figure 46. The power module and drift tube delivers a uniform electric field resulting in resolving powers that are almost twice that of other OTS ion mobility units coupled with the ability to select the electric field strength of the unit at will.

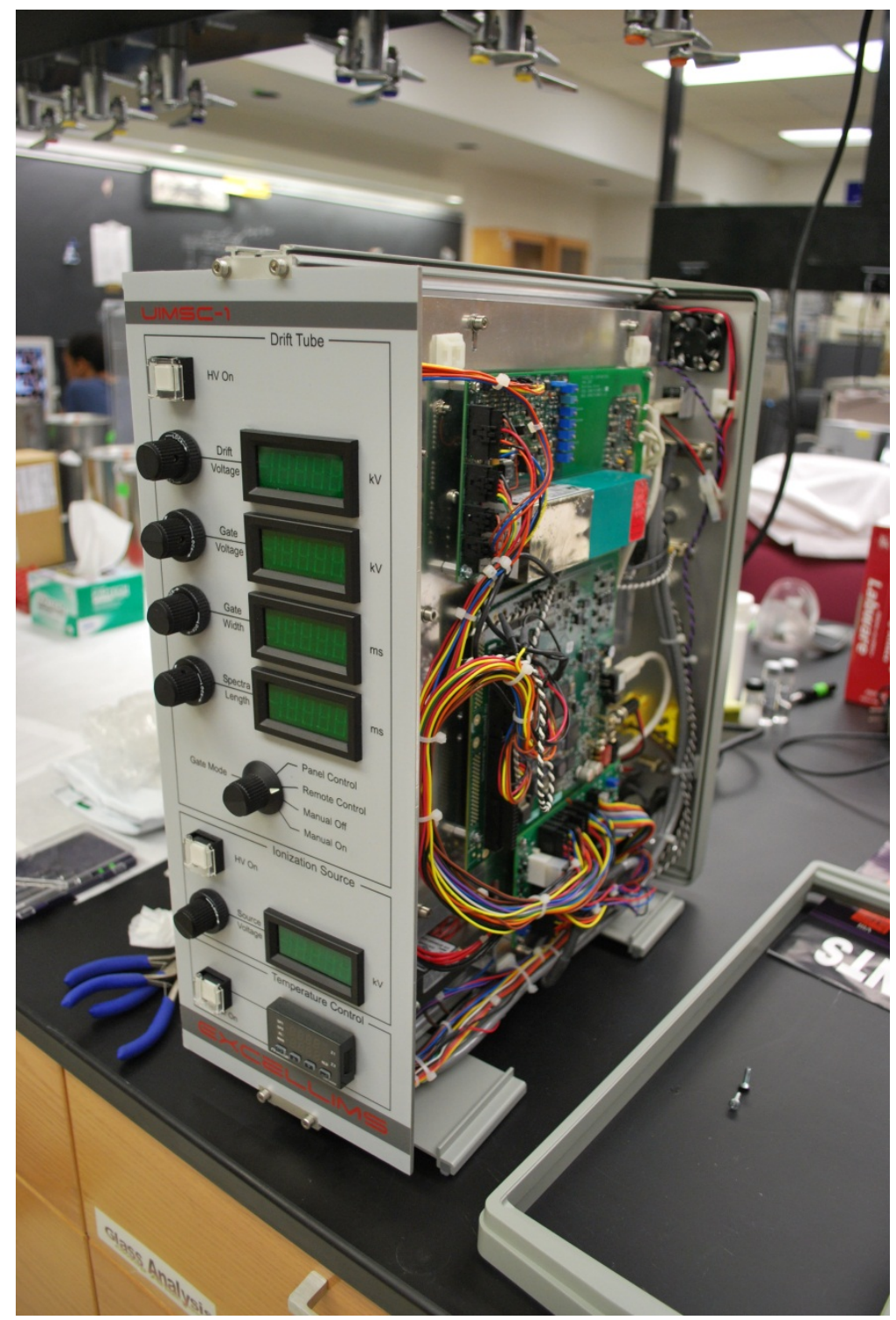

Figure 46: High Voltage Power module for RA4100

The mass spectrometer coupled to the IMS unit has a quadrupole mass selector from Extrel Core Mass Spectrometers (Pittsburgh, Pennsylvania) with a mass range of 5 to 500 
amu and provides unit mass resolution along this mass range. In order to overcome the difficulty of coupling a technique that operates at atmospheric pressure to one that operates under vacuum, a 40L per hour roughing pump is used which backs up a turbo pump displacing 300L per second. The combination of turbo and roughing pump is sufficient to maintain a vacuum of $10^{-6}$ Torr through a $200 \mu \mathrm{m}$ orifice (Figure $47 \mathrm{~b}$ ). As discussed in section Coupling IMS to Mass Spectrometry (pg.28), both IMS and Mass spectrometry are destructive techniques. In order for both techniques to be coupled, a portion of the sample from one technique (IMS) must be transferred to the other technique (MS).

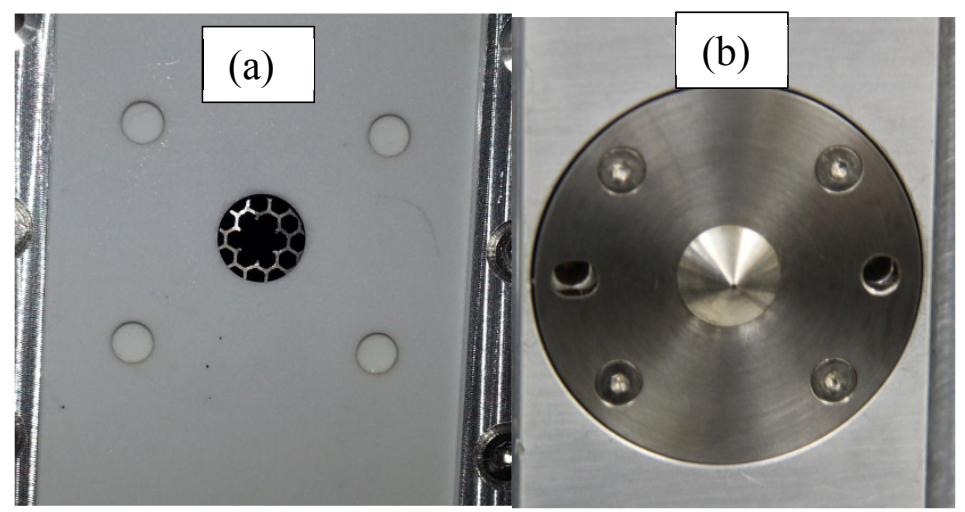

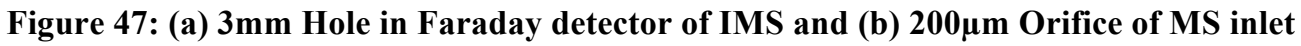

The IMS and MS interface was accomplished by creating a hole in the faraday detector of the IMS, ions would pass undetected through the IMS faraday detector and enter the MS orifice where they would be further separated by mass to charge ratio. The interface allows for the generation of both IMS and MS data simultaneously from the introduction of the same sample as seen in Figure 48. 

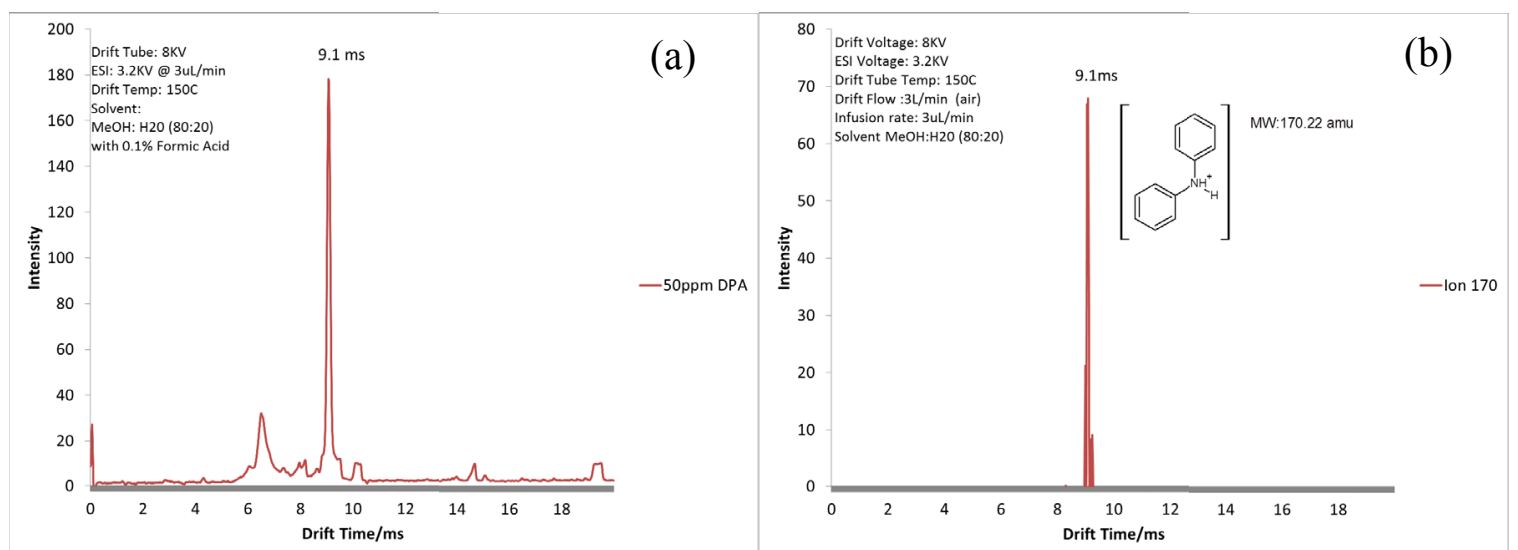

Figure 48: (a) IMS and (b) Single ion plot of Diphenylamine showing the mobility of the protonated $170 \mathrm{~m} / \mathrm{z}$ ion.

The single feature that makes the RA4100 truly chiral is the presence of a modifier chamber. The chamber allows for the introduction of a liquid modifier that is heated and thoroughly mixed with the drift gas of the IMS Figure 49.

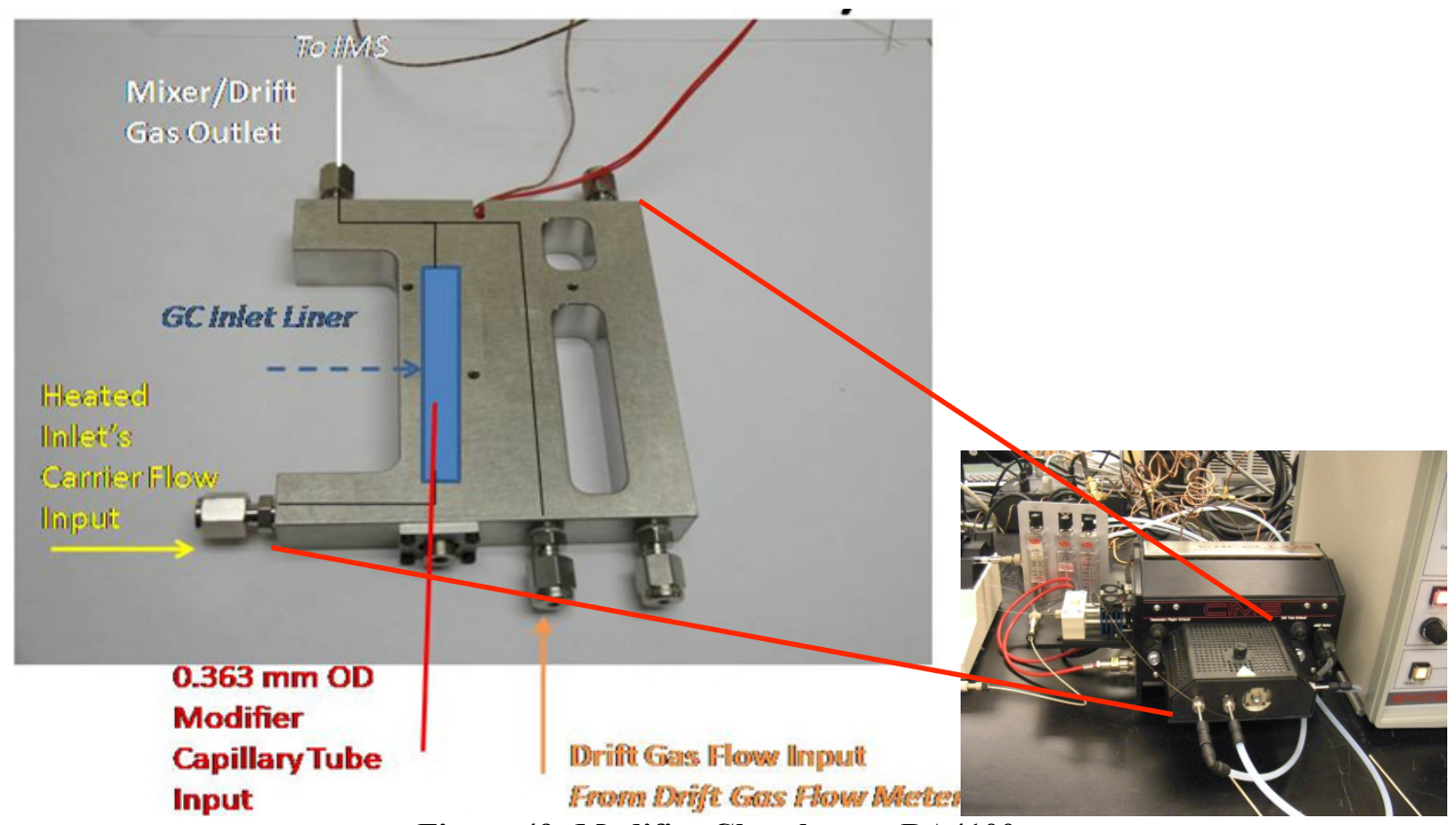

Figure 49: Modifier Chamber on RA4100

The addition of the modifier chamber on this IMS unit would minimize the inefficient introduction observed in the section entitled Experimental Design 
Adaptation of an Ion Mobility Spectrometer for Chiral Separations (pg. 56). The modifier was infused through a $350 \mu \mathrm{m} \mathrm{OD} / 75 \mu \mathrm{m}$ ID capillary tube that was coupled to a separate syringe driven by a syringe pump. The syringe was a $250 \mu \mathrm{L}$ gas tight syringe from Hamilton Company, Reno NV and the syringe pump a Harvard Apparatus 11. With these areas mentioned, the parameters for optimization were laid out, the voltage to be applied to the electrospray ionization source (ESI), the electric field strength and the amount of modifier that was required to result in the desired separation.

\subsubsection{Optimization of the Electrospray Ionization Source}

For all experiments a solvent mixture of methanol (80\%) and water (20\%) was used. The electrospray ionization source of this unit does not have artificial nebulization, the absence of which allows for true ESI conditions as described in the section titled Charge Competition in IMS (pg. 41), however, this also limits the flow rates that may be used. Electrospray ionization flow rates for all experiments were limited to less than $10 \mu \mathrm{L}$ per minute and for most experiments with very few exceptions, $3 \mu \mathrm{L}$ per minute was used as the flow rate. The voltage required for ESI conditions was also investigated, it was observed that voltages below $3000 \mathrm{~V}$ between the ESI tip and counter electrode did not result in electrospray conditions and as a result, no ions were generated or detected. Flow rates above the $10 \mu \mathrm{L}$ per minute had a quenching effect on ion generation, suspected to be as a result of too much solvent entering the ESI and it not being ionized sufficiently. Flow rates within the 3 to $5 \mu \mathrm{L}$ per minute range provided intense ions and steady spectra. In an attempt to improve de-solvation of solvent at the ESI, the gas flow that lead directly 
to the ESI (de-solvation gas) was varied independently of the drift gas to determine its effect if any, on ion intensity. Experiments revealed that the de-solvation gas did have an effect on ion intensity. Figure 50 shows the effect of varying desolvation gas flow rates when cocaine was used as the analyte, the ion intensity gradually increases and appears to plateau at $700 \mathrm{ml}$ per minute of de-solvation gas (air) when using a flow rate of $3 \mu \mathrm{L}$ per minute of sample being delivered to the ESI.

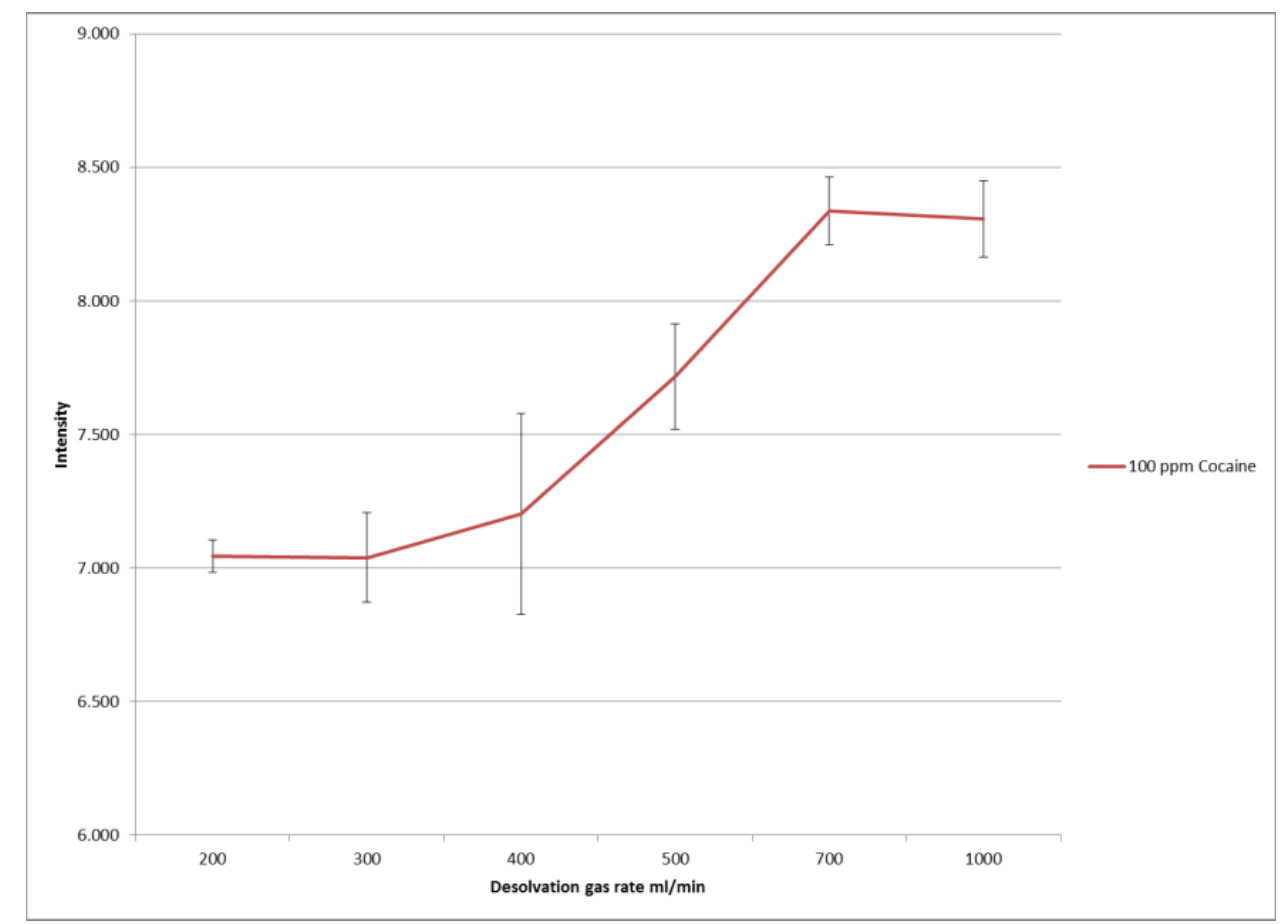

Figure 50: Effect of desolvation gas on ion intensity

It was therefore decided to maintain flow rates between 3 and $5 \mu \mathrm{L}$ per minute, ESI voltage above $3000 \mathrm{~V}$ and de-solvation gas above $700 \mathrm{ml}$ per minute in order to obtain acceptable ion mobility and mass spectra. Immediately after ions are generated at the ESI source, they are regulated by the IMS gate, which is also adjustable on the RA4100. It was previously shown in Figure 37 that a reduction in gate pulse increases overall resolution between analytes. The increase in resolution is as a result of an increase in 
resolving power from shorter more compact bands of the created ion swarm being allowed to enter the IMS drift tube. For ESI-IMS on the RA4100 this was also true, the instrument performed in a predictable manner providing higher resolving powers at reduced gate pulse settings Figure 51. The instrument was able to produce a maximum resolving power of 75 again using cocaine as the analyte at a gate pulse of $75 \mu$ s (Figure $51)$.

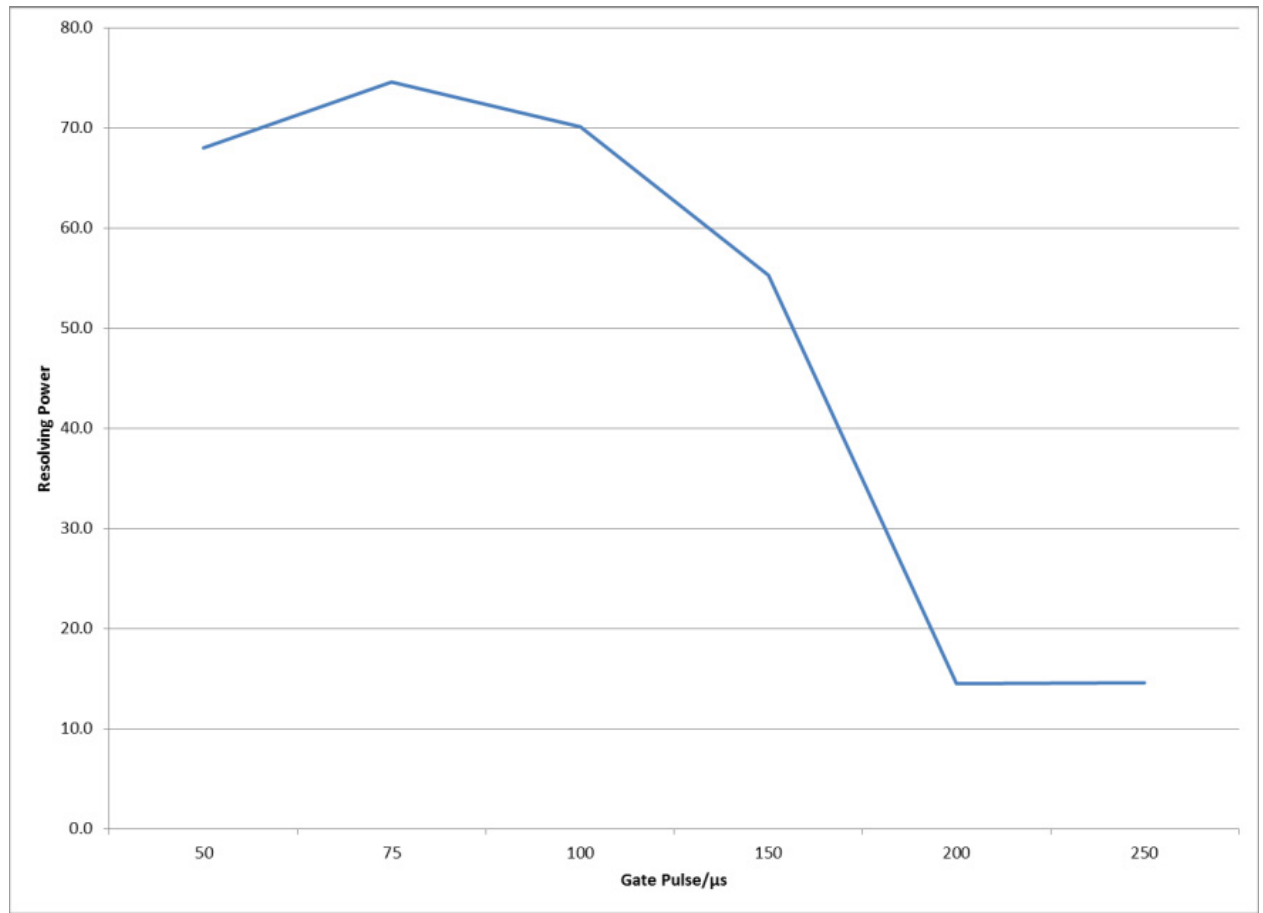

Figure 51: Effect of gate pulse on resolving power on RA4100

With both the OTS Ionscan 400B and the RA4100 ion mobility unit, an increase in gate pulse reduced the overall resolving power of the analytes. Of interest was the fact that the shape of the decline for this trend on the RA4100 followed a convex curve (Figure 51) as opposed to the concave profile of the Ionscan (Figure 37). The different profiles dictate the inherent design of either instrument where the RA4100 is able to produce a higher and more consistent resolving power over a variety of gate pulses as opposed to the rapid 
and immediate decay in resolution observed on the Ionscan 400B. The resolving power profile is indicative of the improved drift tube capabilities offered by the RA4100 ion mobility system. For all experiments a gate pulse of 80 microseconds was used.

\subsubsection{Optimization of the Drift Gas Flow and Tube Potential}

The drift tube of the RA4100 system is approximately $10 \mathrm{~cm}$ in length and is able to have applied potentials of up to 10,000 volts $(1000 \mathrm{~V} / \mathrm{cm})$. The effect of varying the applied voltage across the RA4100's drift tube was investigated and found to have a significant impact on the resolving power and intensity of ions being separated. Again, cocaine was used to conduct these tests and the results displayed in Figure 52.

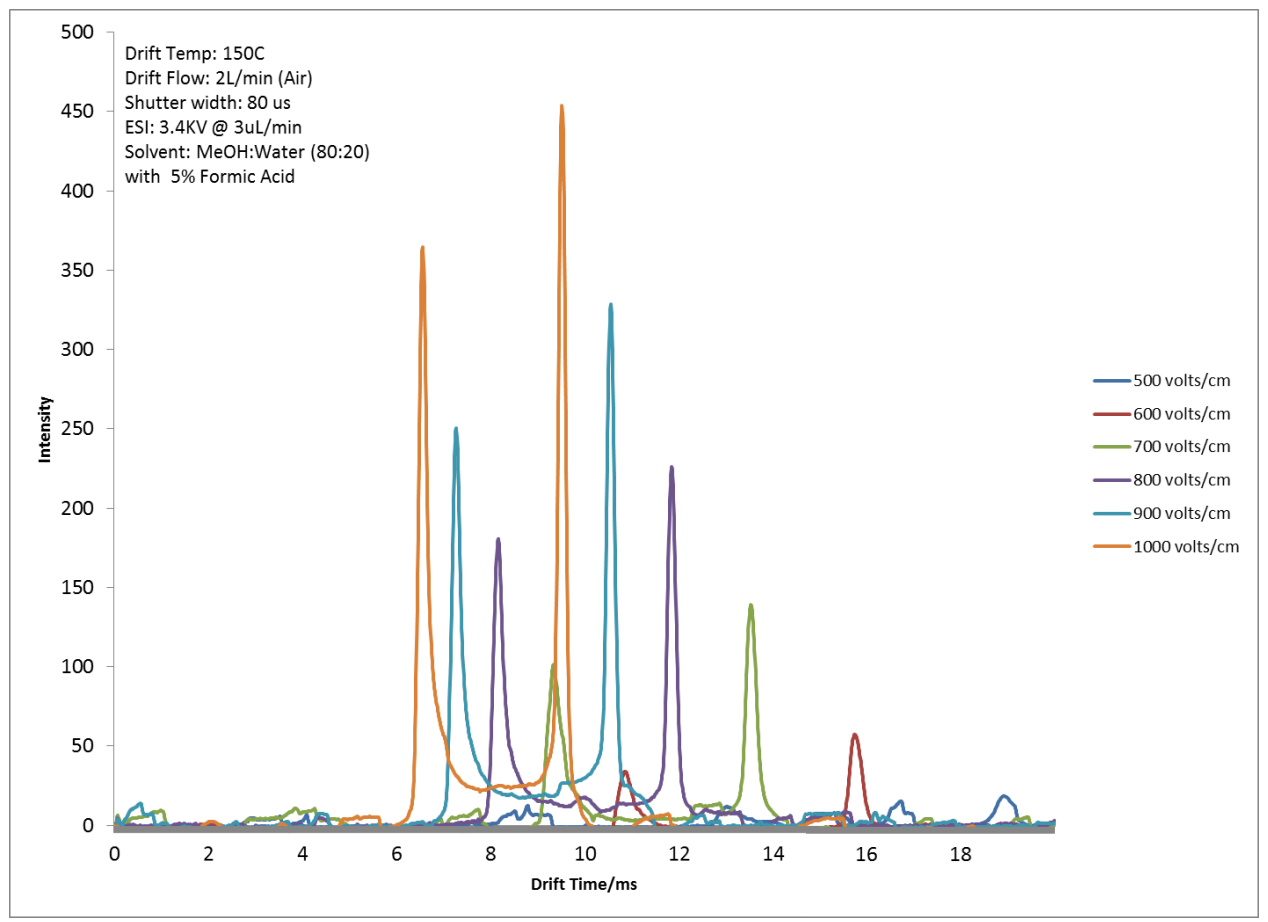

Figure 52: Effect of electric field strength on analytes in RA4100 
There is a direct relationship between the electric field strength and the intensity of analyte detected. The relationship between field strength and ion intensity is as a result of the more powerful electric field being able to focus more ions and propel them down the drift tube towards the faraday plate to be detected, resulting in a higher ion current and hence higher signal as seen in Figure 53. The only caveat to the utilization of higher drift tube potential is the possibility of causing damage to the high voltage components of the RA4100 as a result of arcing due to dielectric breakdown. The risk of damaging the components therefore limited most experiments to be conducted to the slightly lower potential of 8000 Volts providing an electric field strength of $800 \mathrm{~V} / \mathrm{cm}$.

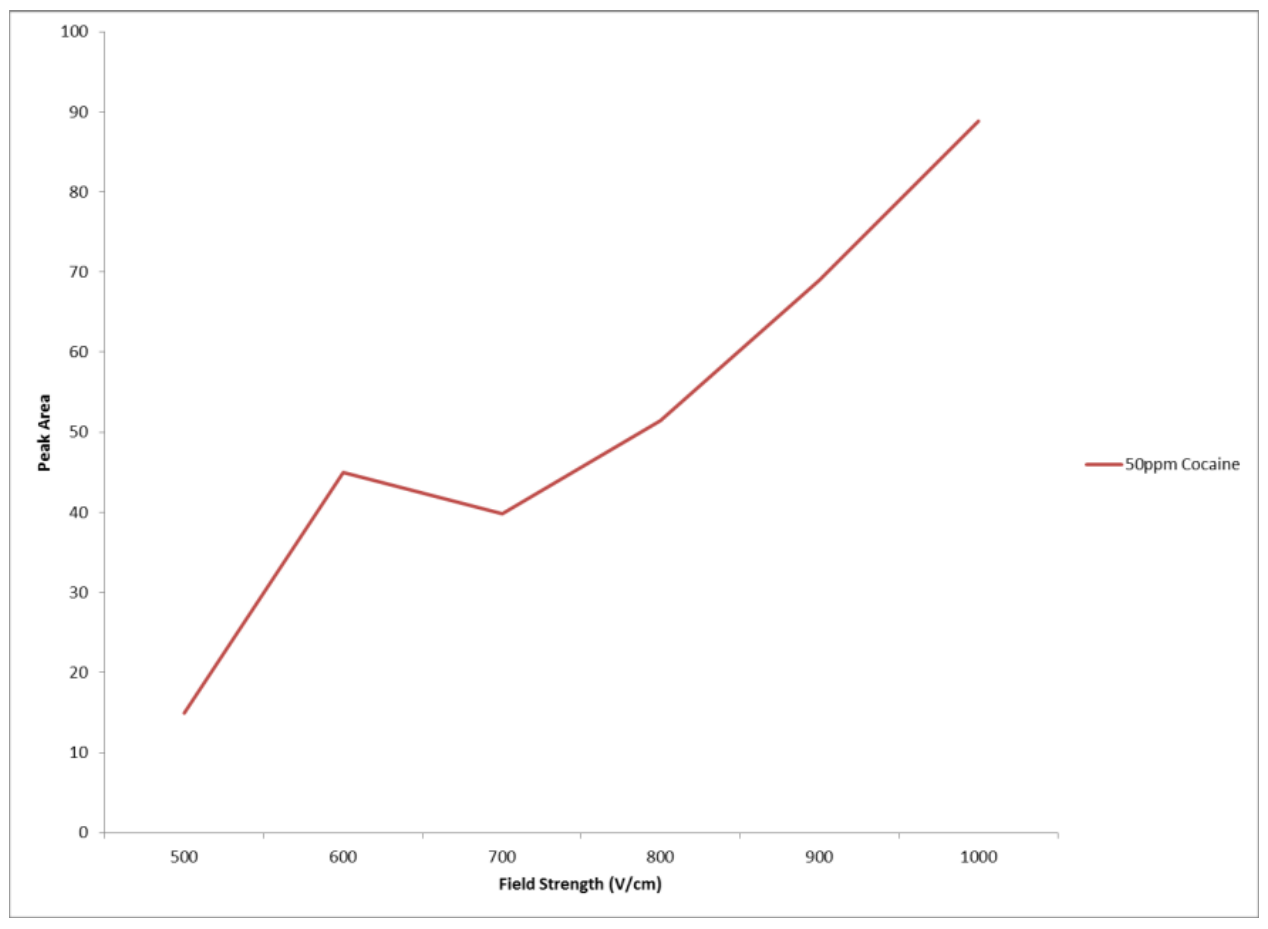

Figure 53: Effect of Electric field strength on ion intensity

Temperature has always played a significant role in ion mobility experiments. No less so with the RA4100 as the electrospray solvent used introduced a significant quantity of water $(20 \% \mathrm{v} / \mathrm{v})$ being infused into the drift tube. The high amount of water used required 
significantly high drift tube temperatures to prevent excessive water clusters from forming within the drift tube that would retard ions and steal charge, causing a loss in resolution and lower signal intensity. One interesting observation made with the RA4100 was that the drift tube design required a relatively high drift gas flow rate. Typically the drift gas composition is altered to affect the mobility of ions, however, the mobility of ions were observed to be altered by changing the drift gas flow rates on the RA4100. Figure 54 shows a mixture of Caffeine, Diazepam and Cocaine all analyzed by ESI-IMS on the RA4100 utilizing different drift gas flow rates of air. The figure shows that as the drift gas flow rate increases, the overall separation between analytes (mobility) increases providing increased resolution. The separation is crucial for analytes such as diazepam and cocaine that have very close mobility values of $\left(1.211 \mathrm{~cm}^{2} \mathrm{~V}^{-1} \mathrm{~s}^{-1}\right)$ and $\left(1.160 \mathrm{~cm}^{2} \mathrm{~V}^{-1} \mathrm{~s}^{-}\right.$ $\left.{ }^{1}\right)$ respectively. These analytes showed no separation using low drift gas flow rates of $200 \mathrm{ml} /$ minute and near complete separation at a flow rate of $2000 \mathrm{ml} / \mathrm{min}$ as shown in Figure 54. 


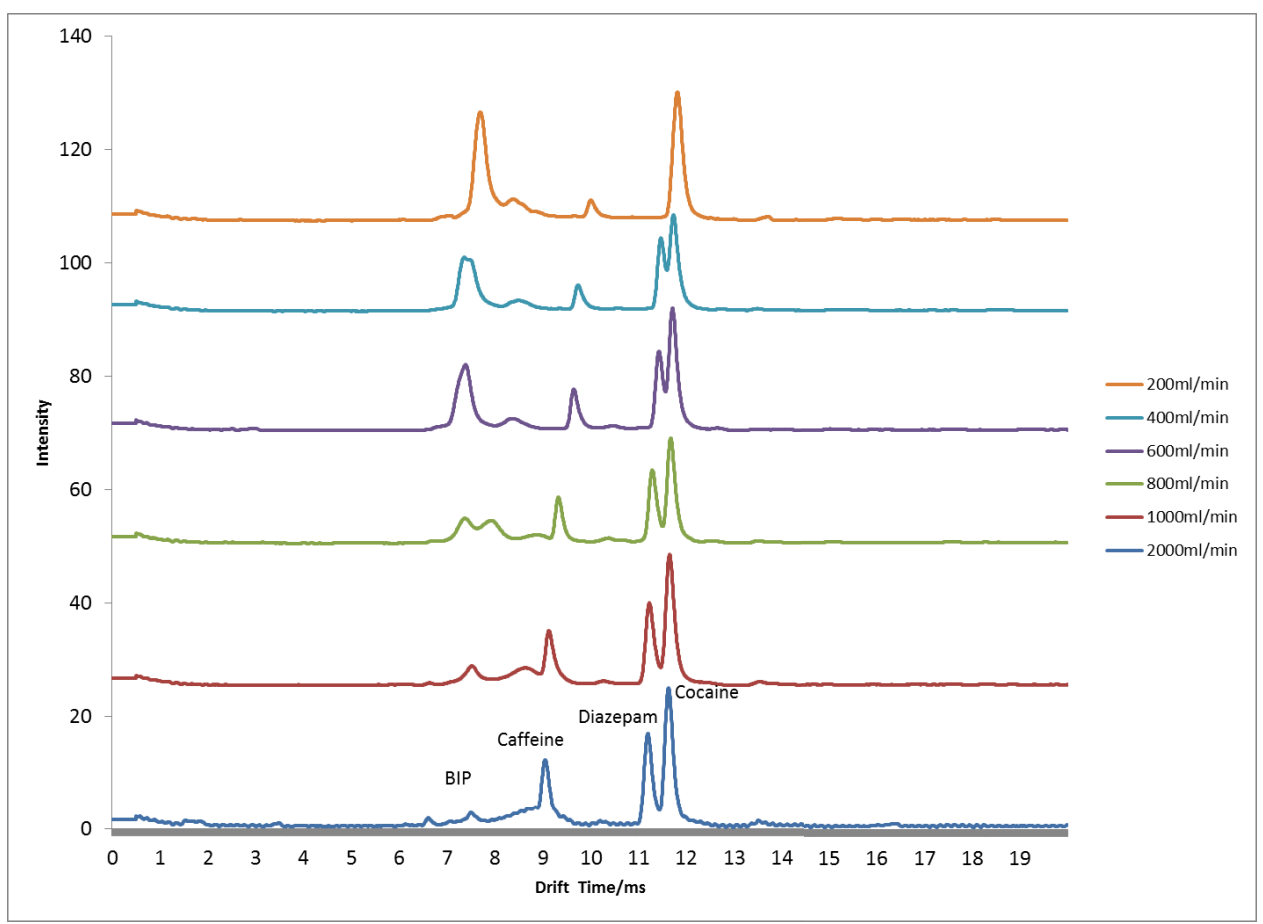

Figure 54: Effect of RA4100 drift gas flow rate on ion mobility of a mixture of analytes The reason for this change in mobility and overall improvement in separation is not fully understood. The hypothesis being that the higher flow rate drift gas is able to remove more neutral solvent molecules that retard the ions, from within the drift tube. Though, mass spectrometric analysis was unable to confirm the hypothesis for improved separation using higher drift gas flow as there was no difference in the mass to charge ratio of the ions detected at the lower drift tube flow rates versus those at the higher drift tube flow rates. However, the dominance of the solvent peak (background ion peak) at the lower drift tube flow rates and the lack of the background ion peak (BIP) at the higher drift tube flow rate does reinforce the hypothesis since it implies less overall solvent molecules within the drift tube. From these observations drift gas flow rates of $2000 \mathrm{ml} / \mathrm{min}$ were used for all experiments on the RA4100. 


\subsubsection{Utilizing a Quadrupole Mass Spectrometer as an IMS detector}

The mass spectrometer used on the RA4100 was designed by a third party vendor, Extrel Core Mass Spectrometers from Pittsburgh Pennsylvania. The QC-150 has a mass range from 5 to $500 \mathrm{amu}$ and utilizes a quadrupole mass selector. Similar to mass spectrometers used on liquid chromatography systems, the QC-150 did not utilize a filament that will create its own ions, rather it attracts and guides ions created externally to the instrument through a series of cones from an inlet into the mass selector. However, unlike most mass spectrometers utilized for liquid chromatography applications, the QC-150 does not share a similar high mass range of 2000 amu. While the lower mass range is suitable for ion mobility measurements as most analytes will be small volatile to non-volatile molecules, it did create initial challenges when first utilizing and tuning the instrument. Typical tuning solutions used on LC-MS systems include tetramethylammonium bromide, tetrabutylammonium bromide, tetrakis(decyl)ammonium bromide and perfluoroalklphophazine producing mass spectra that ranges from $500 \mathrm{amu}$ to $2000 \mathrm{amu}$. These spectra are outside the range of the QC-150 and so could not be used. Smaller molecules such as perfluorotributylamine (PFTBA) also known as FC43 used for GC applications, which would be on a similar scale to IMS could also not be used. Perfluorotributylamine requires electron impact to create its mass fragments that range from 69 to 502 amu, as the QC-150 did not possess a filament for electron impact it eliminated the use of the standard PFTBA. The alternative was to use polyethylene glycols as these could be electro sprayed similar to the bromide mixtures used on LC-MS systems and produce a mass spectrum in the range of the QC-150 as seen in Figure 55. Polyethylene glycol (PEG-400) was electro sprayed into the RA4100 in a solution of 
$80 \%$ methanol and $20 \%$ water, the spectrum detected could then be used to adjust mass assignments and peak resolution of the instrument based on the known masses of the PEG-400 (carbowax).

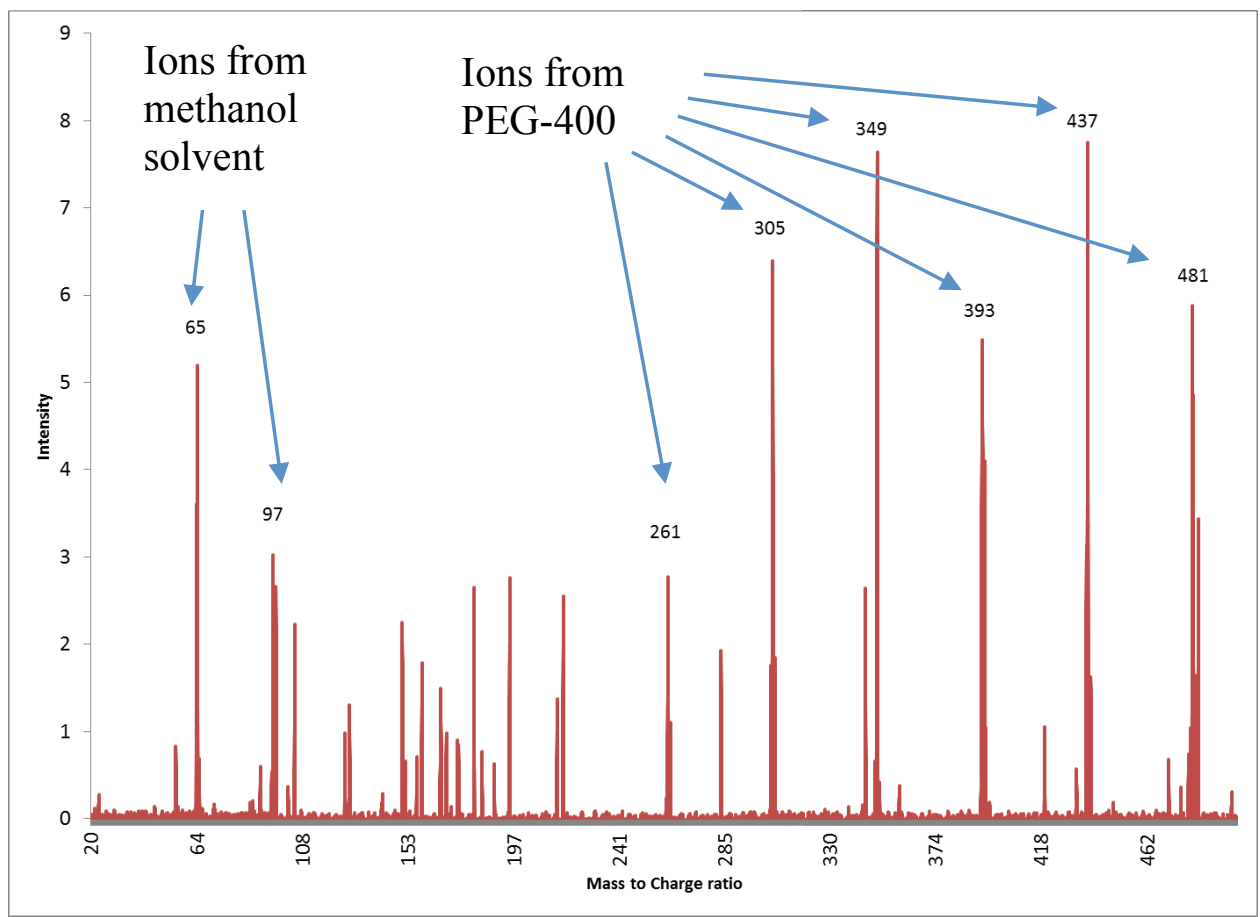

Figure 55: Full scan mass spectrum of PEG 400

The utilization of PEG-400 resulted in a tuned QC-150 that could then be used for analytical work. The spectra observed for the PEG- 400 is as a resulted of sodiated adducts of polyethylene glycol $\left(\mathrm{M}+\mathrm{Na}^{+}\right)$and the range of masses from different polymer lengths of the polyethylene glycol being sodiated, where each repeating polymer unit is $44 \mathrm{amu}$ (Figure 56). Ions 65 and 97 were protonated adducts of methanol $\left(\mathrm{CH}_{3} \mathrm{OH}\right)_{\mathrm{n}} \mathrm{H}^{+}$. 


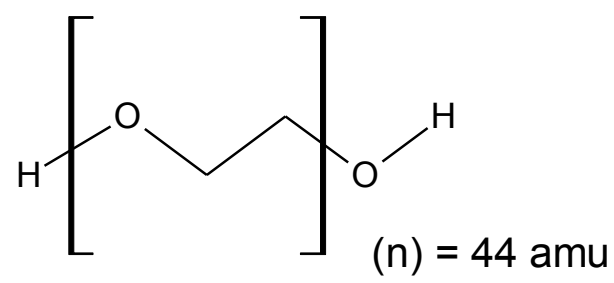

Figure 56: Repeating unit of Polyethylene glycol (PEG) 400 polymer observed on mass spectrum

The observed mass can be simply explained by changes in 44 amu as a loss of one repeating unit from the polymer to produce the entire mass range as shown in Table 3.

Table 3: Masses of Polyethylene glycol (PEG) detected by Mass Spectrometer

\begin{tabular}{|c|c|c|}
\hline $\begin{array}{c}\text { Observed } \\
\text { Mass to } \\
\text { Charge }\end{array}$ & $\begin{array}{c}\text { Actual Mass } \\
\text { to Charge }\end{array}$ & $\begin{array}{c}\text { repeating } \\
\text { units (n) }\end{array}$ \\
\hline 261.5 & 261.26 & 5 \\
\hline 305 & 305.31 & 6 \\
\hline 349.5 & 349.37 & 7 \\
\hline 393.3 & 393.42 & 8 \\
\hline 437.5 & 437.47 & 9 \\
\hline 481.1 & 481.53 & 10 \\
\hline
\end{tabular}

Though mass spectrometrists have used PEG's for years, special thanks goes to Dr. Bert Lynn at the University of Kentucky for a targeted suggestion that saved what would have otherwise been an extended search through numerous research articles [107]. Of special novelty was the application of PEG's to ion mobility work, though the PEG-400 allowed the calibration of the QC-150 mass spectrometer, it simultaneously allowed for the performance check of the ion mobility system as well. Figure 57 shows the overlay of the ions detected from the PEG 400 as observed by the RA4100 ion mobility and mass spectrometer. 


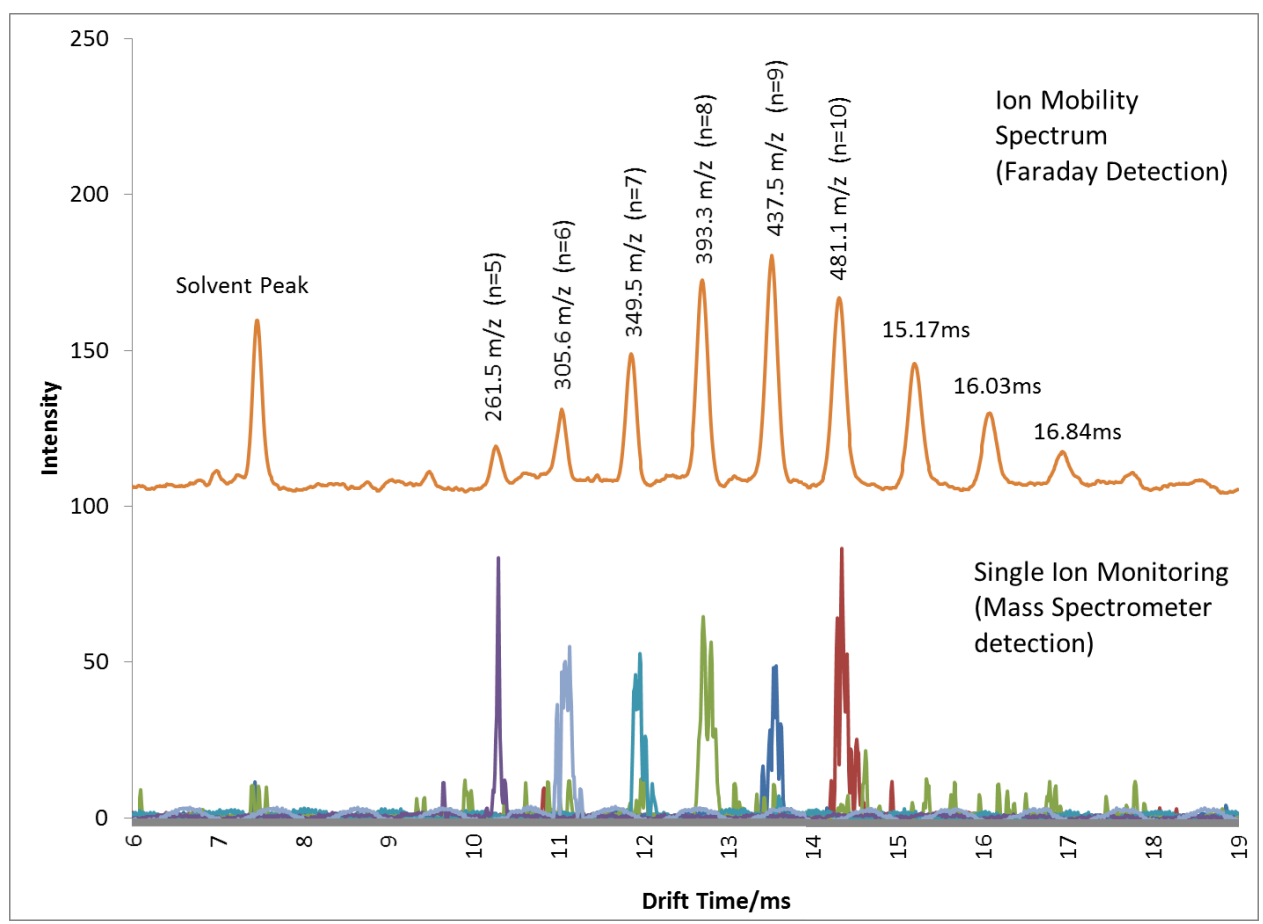

Figure 57: Overlay of Ion Mobility and Single ion monitoring of PEG 400 on RA4100

Figure 57 not only shows each ion of the PEG polymer, but also reveals that each of these ions has a different mobility. The ion mobility goes further in being able to detect what appears to be ions from polymers with repeating units of 11,12 and 13 observed at 15.17 , 16.03 and $16.84 \mathrm{~ms}$ respectively (Figure 57). These ions would have expected mass to charge ratios of 525, 569 and $613 \mathrm{~m} / \mathrm{z}$ respectively, above the range of the QC-150 and as a result not observed in the mass spectrum. Of interest was that each of these ions produced drift times that were $0.8 \mathrm{~ms}$ apart, indicating that the electric field within the drift tube of the RA4100 was uniform along its entire separation range.

The requirement of accurate mass assignment or mass calibration while performing atmospheric ionization of ions, is often times more stringent than when conducting work with electron impact ionization. The occurrence of adducted species and other phenomena during atmospheric ionization of samples often produces unexpected mass 
shifts. These mass shifts can lead to inaccurate assumptions when dealing with analytes of unknown origin. Case in point, Figure 58 shows the mass spectrum of cocaine.

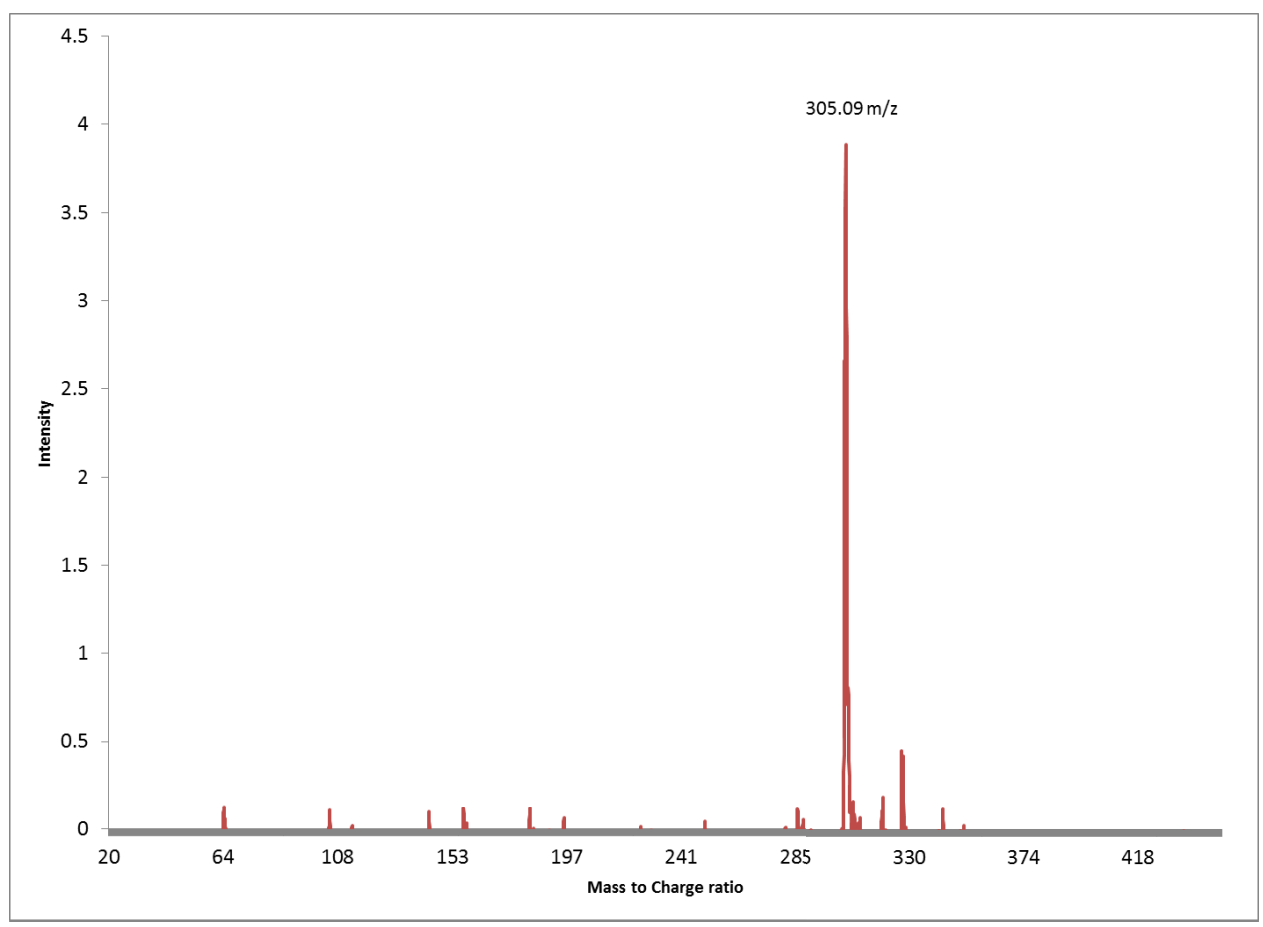

Figure 58: Mass spectrum of Cocaine obtained on RA4100 showing inaccurate mass assignment

The salient peak observed has a mass assignment of $305 \mathrm{~m} / \mathrm{z}$. The assumption of the 305 peak being correct would have led to an intensive search to determine the possible adducting species and structure of protonated cocaine which is expected to be 304.36 $\mathrm{m} / \mathrm{z}$, however the $305 \mathrm{~m} / \mathrm{z}$ being obtained was found to be as a result of inaccurate mass assignment by the RA4100 mass spectrometer. The inaccurate mass assignment was adjusted by utilizing the PEG 400 as shown in Table 3 and Figure 57 to obtain the accurate mass of any analyte being obtained. On the basis of the data in Table 3, the correlation coefficient of observed mass assignments versus the actual mass of analytes is 1 as show in Figure 59. 


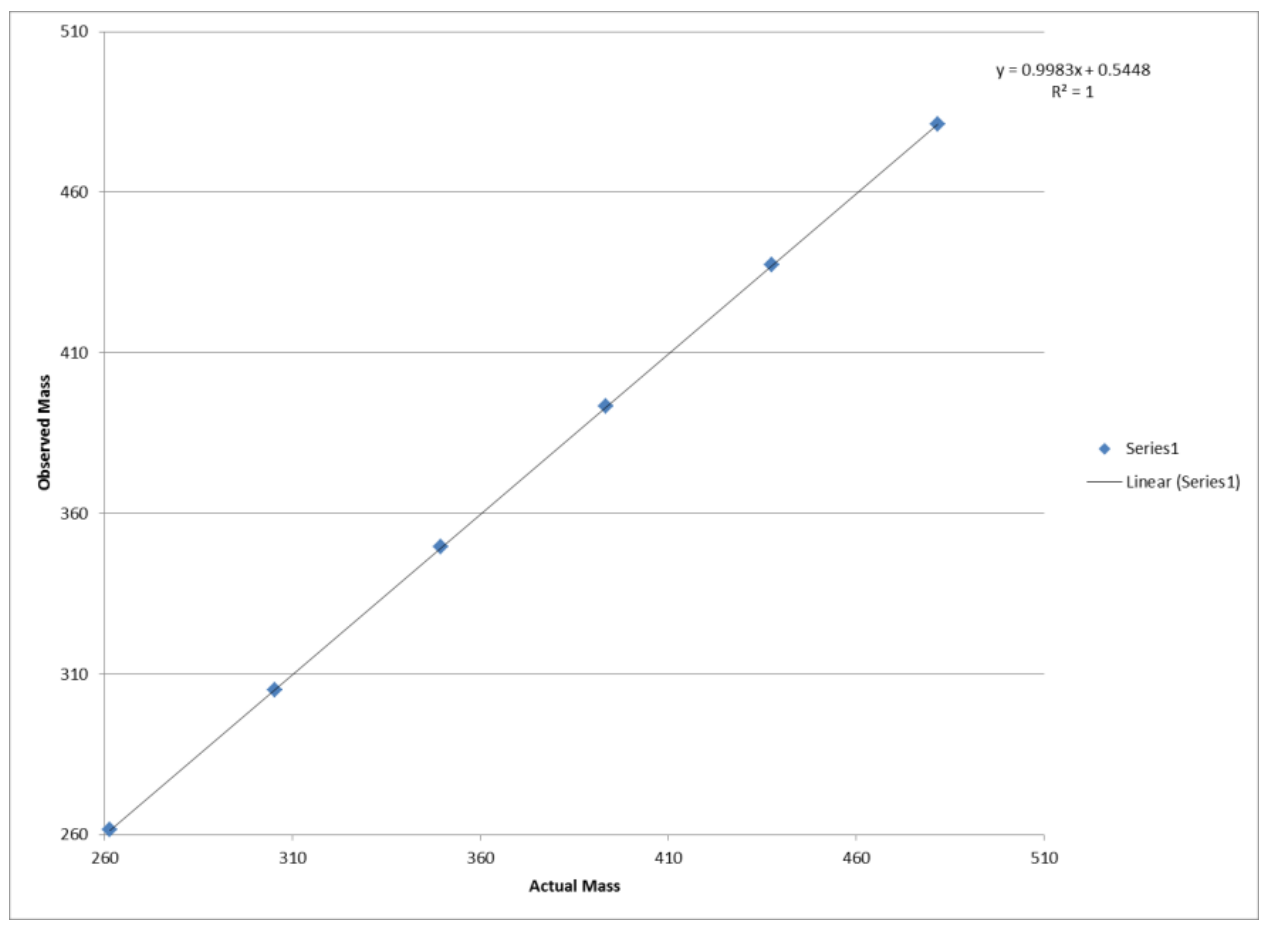

Figure 59: Correlation of observed versus actual mass of analytes on calibrated RA4100

\subsubsection{Analysis of Multiple Component Mixtures Utilizing ESI-IMS-MS}

The RA4100 was conceived as a fully functional analytical instrument, with the ability to analyze and separate complex mixtures and obtain results analogous to that obtained from either GC or LC separations. In order to achieve this status, the RA4100 ESI-IMS, must be able to simultaneously ionize and separate mixtures, which previously had been a challenging task. The previous section introduced some of the steps taken during ionization with electrospray to allow for the simultaneous analysis of mixtures. The next section will go into detail of the experimental approach taken and the hypothesis dictating why these techniques are required for successful analysis of complex mixtures utilizing ESI-IMS. The appropriate solvent in ESI-IMS can enhance the ionization of analytes, allowing simultaneous ionization of mixtures of analytes. As IMS is an atmospheric gas 
phase separation technique, analytes are bombarded by the surrounding atmosphere (drift gas) which improves separation by allowing differentiation of analytes on the basis of their collision cross sectional area as well as mass to charge ratio. However, the drift gas also steals charge from ionized species, resulting in ions only surviving a few milliseconds within the drift tube. In addition to this, each ion competes with neighboring ions for charge. Often resulting in only those ions possessing relatively high proton affinities, being the dominant ions detected. Selection of the appropriate solvent system allows ions with low proton affinities the opportunity to be ionized alongside those ions with much higher proton affinities. It has been previously reported that simultaneous ionization of analytes through ESI-IMS may occur when analyzing compounds with similar proton affinities, such ionization does not readily occur with analytes possessing differing proton affinities. In order for such analysis, it was found necessary to have acids added to solute solutions above a certain concentration for positive mode analysis. Utilization of acids allowed simultaneous ionization of analytes referred to as "multiplexing" [108]. Figure 60 shows a mixture of Caffeine, Diazepam and Cocaine when analyzed using a methanol:water mixture with varying concentrations of formic acid. With higher acid concentration, all analytes are ionized whereas the weaker acid mix resulted in only cocaine, the analyte with the highest proton affinity being detected (Table 4). 
Table 4: Ionization energies of common ESI solvents and analytes.

\begin{tabular}{|c|c|c|c|}
\hline Solvent & $\begin{array}{l}\text { Proton Affinity } \\
(\mathrm{kJ} / \mathrm{mol})\end{array}$ & $\begin{array}{l}\text { Gas Phase Basicity } \\
(\mathrm{kJ} / \mathrm{mol})\end{array}$ & $\begin{array}{l}\text { Ionization energy } \\
(\mathrm{eV})\end{array}$ \\
\hline Methanol & 754.3 & 724.5 & 10.84 \\
\hline Water & 691 & 660 & 12.62 \\
\hline Acetonitrile & 779.2 & 748 & 12.20 \\
\hline Formic acid & 742 & 710 & 11.33 \\
\hline Cocaine & 930 & N/A & N/A \\
\hline Caffeine & 900 & N/A & 7.95 \\
\hline Diazepam & N/A & N/A & N/A \\
\hline
\end{tabular}

The competitive ionization has been modeled by one author to explain its occurrence when using electrospray ionization sources [109]. It was observed that in order for mixtures of analytes dissolved in the same solvent to be ionized, an acid concentration (modifier) above $0.4 \%(\mathrm{v} / \mathrm{v})$ was necessary. Other peer reviewed literature has reported that as much as $5 \%$ modifier is required to prevent problems with ionization for electrospray ionization [110].

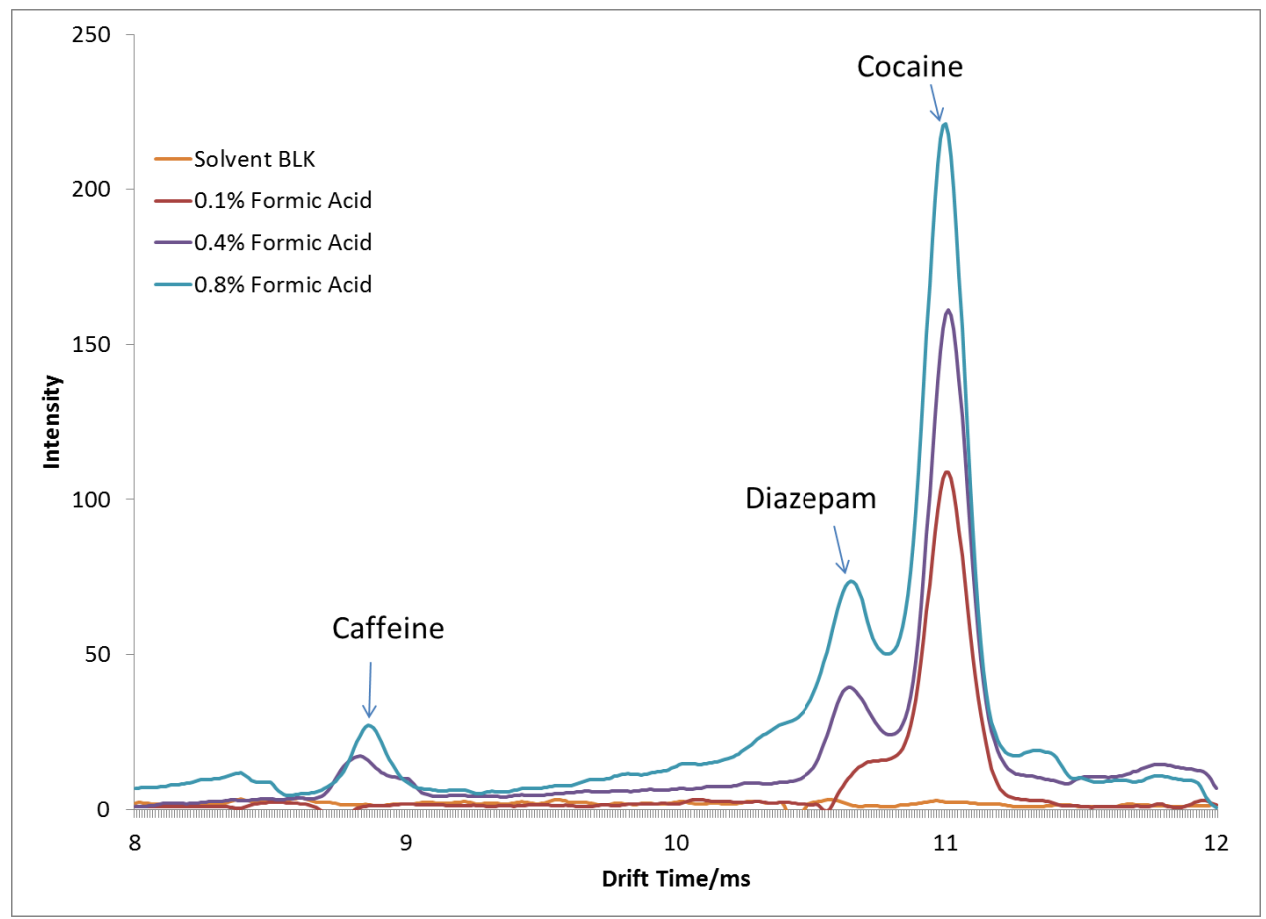

Figure 60: Effect of acid strength on detection of analytes 
Solvents such as methanol are more easily ionized than acetonitrile, as a consequence of the differences in surface tensions that makes spraying of acetonitrile by ESI more difficult [47]. The current experiments revealed the optimal spray conditions when utilizing varying mixtures of methanol and water as the electrospray solvent (Figure 61).

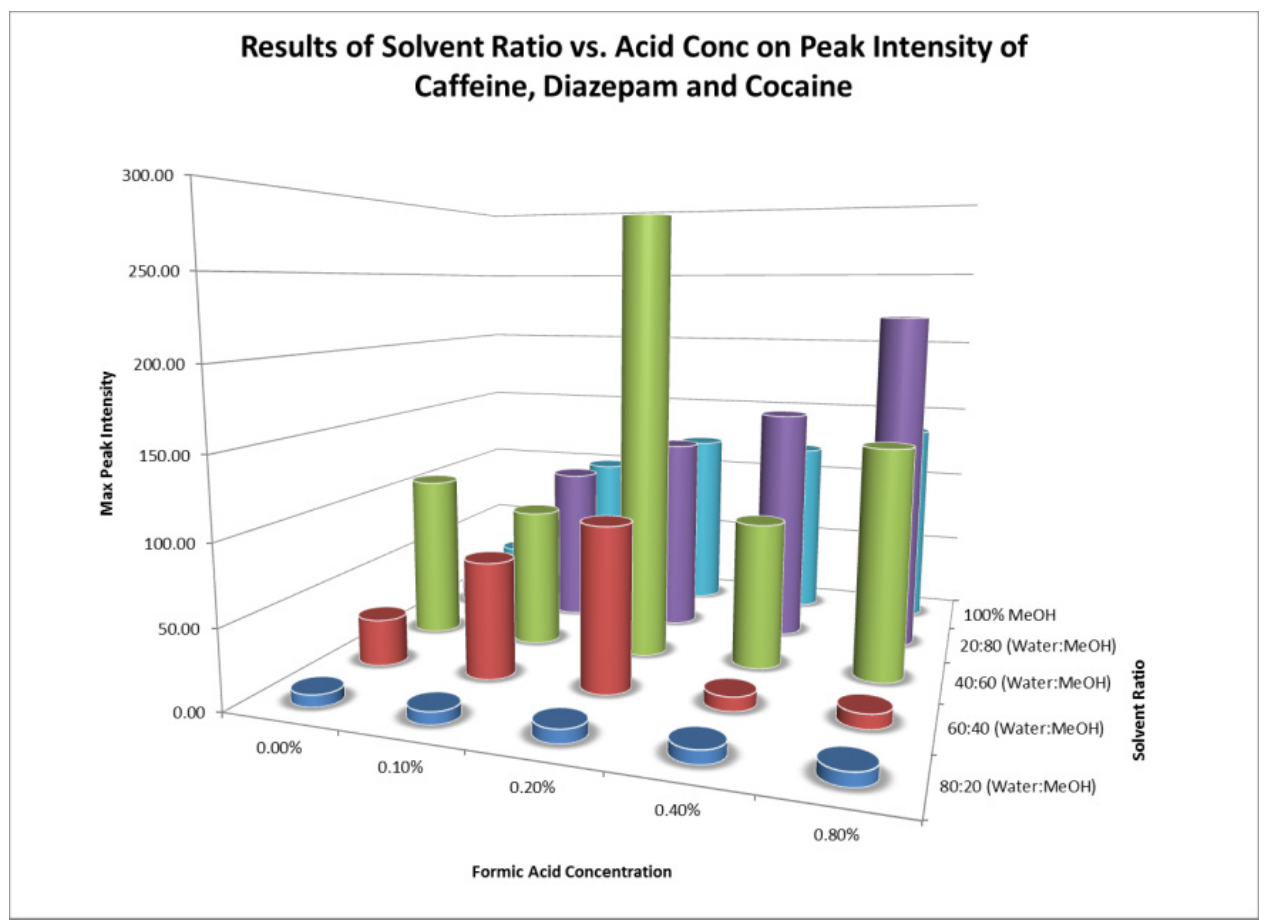

Figure 61: 3-D plot of solvent effect on analyte intensity

Optimal conditions were realized by using either a 60:40 or 80:20 (Methanol:Water) solution. High formic acid concentrations improved overall signal intensities as previously shown (Figure 60). It was observed that higher concentrations of water either inhibited ionization or resulted in ESI spray that was unstable. Most likely because of the higher surface tension of water that requires higher electrospray voltages in order to form the Taylor cone that results in stable spray characteristics [47].

It was also observed that high acid concentrations resulted in wider more diffused analyte peaks, though this same high acid concentration also provided greater signal intensities 
and simultaneous ionization of analytes. Table 5 reveals experimental data for the peak width at half maximum (FWHM) for several analytes with varying concentrations of acid modifier. Higher modifier concentrations resulted in wider peaks (higher FWHM) that reduced the overall resolving power of the technique.

Table 5: Full width half maximum (FWHM) of peaks obtained with different solvent systems of analytes at $50 \mathrm{ng} / \mu \mathrm{L}$ on ESI-IMS-MS

\begin{tabular}{|l|c|c|c|c|c|c|}
\hline & \multicolumn{3}{|c}{ Methanol:Water (60:40) } & \multicolumn{3}{c|}{ Methanol:Water (80:20) } \\
\hline Formic Acid & Caffeine & Diazepam & Cocaine & Caffeine & Diazepam & Cocaine \\
\hline $0.40 \%$ & N/D & 0.173 & 0.168 & 0.195 & 0.204 & 0.170 \\
\hline $0.80 \%$ & 0.173 & 0.249 & 0.185 & 0.163 & 0.230 & 0.179 \\
\hline $1.00 \%$ & 0.199 & 0.233 & 0.194 & 0.170 & 0.261 & 0.226 \\
\hline $1.40 \%$ & 0.227 & 0.259 & 0.220 & 0.228 & 0.244 & 0.212 \\
\hline $1.80 \%$ & 0.223 & 0.261 & 0.307 & 0.271 & 0.258 & 0.258 \\
\hline $2.50 \%$ & 0.218 & 0.279 & 0.243 & 0.261 & 0.277 & 0.253 \\
\hline
\end{tabular}

On the basis of these observations, the concentration of the analyte to be detected had to be carefully considered when preparing a solvent-solute mixture. The consideration of the amount of acid used ensured effective ionization while maintaining the best resolving power (lowest FWHM) during ESI-IMS analysis. Different solvents also determined the species of analyte ion being formed and detected. Solvents determined whether the ionic species would be sodium adducted, ammonium adducted or chlorinated. Various studies were conducted involving both positive and negative mode ionization utilizing different solvent chemistries. In traditional ${ }^{63} \mathrm{Ni}$ based IMS units, the drift gas is infused or doped with a volatile species which generates a reactant ion peak (RIP). These dopants are typically ammonia for positive mode analysis and methylene chloride for the negative mode [111]. However, in ESI based IMS units such as the RA4100, dopants are not used as ionization of analytes occurs within the solvent before the analytes enter the gas phase. 
In ${ }^{63} \mathrm{Ni}$ based IMS systems, ionization occurs after the analytes are desorbed into the gas phase, allowing the dopant to play an active role in the ionization process. The ionization is done by the dopant reacting with the beta particles from the radioactive nickel which forms a reactant ion that in turn ionizes the desorbed neutral analytes. While in ESI, the analytes are ionized within solution, forming ions of the solvent and analytes simultaneously before either enters the gas phase. As a result, dopants do not play a role during the electrospray ionization process as the ions have already formed. Rather than the presence of a reactant ion peak (RIP) formed from the dopant in ${ }^{63} \mathrm{Ni}$ based IMS systems, electrospray ion mobility shows a background ion peak (BIP) [79], which is a direct result of ion clusters formed from the electrospray solvent (Figure 62). The type of background ion peak created can be altered based on the chemistry of the solution being sprayed. Figure 62 shows the full scan mass spectrum, single ion monitoring and ion mobility of an 80:20 methanol:water solution sprayed in positive mode. The ions of 65 $\mathrm{m} / \mathrm{z}\left(\mathrm{CH}_{3} \mathrm{OH}\right)_{2} \mathrm{H}^{+}$and $97 \mathrm{~m} / \mathrm{z}\left(\mathrm{CH}_{3} \mathrm{OH}\right)_{3} \mathrm{H}^{+}$are the most dominant due to the formation of methanol clusters. 


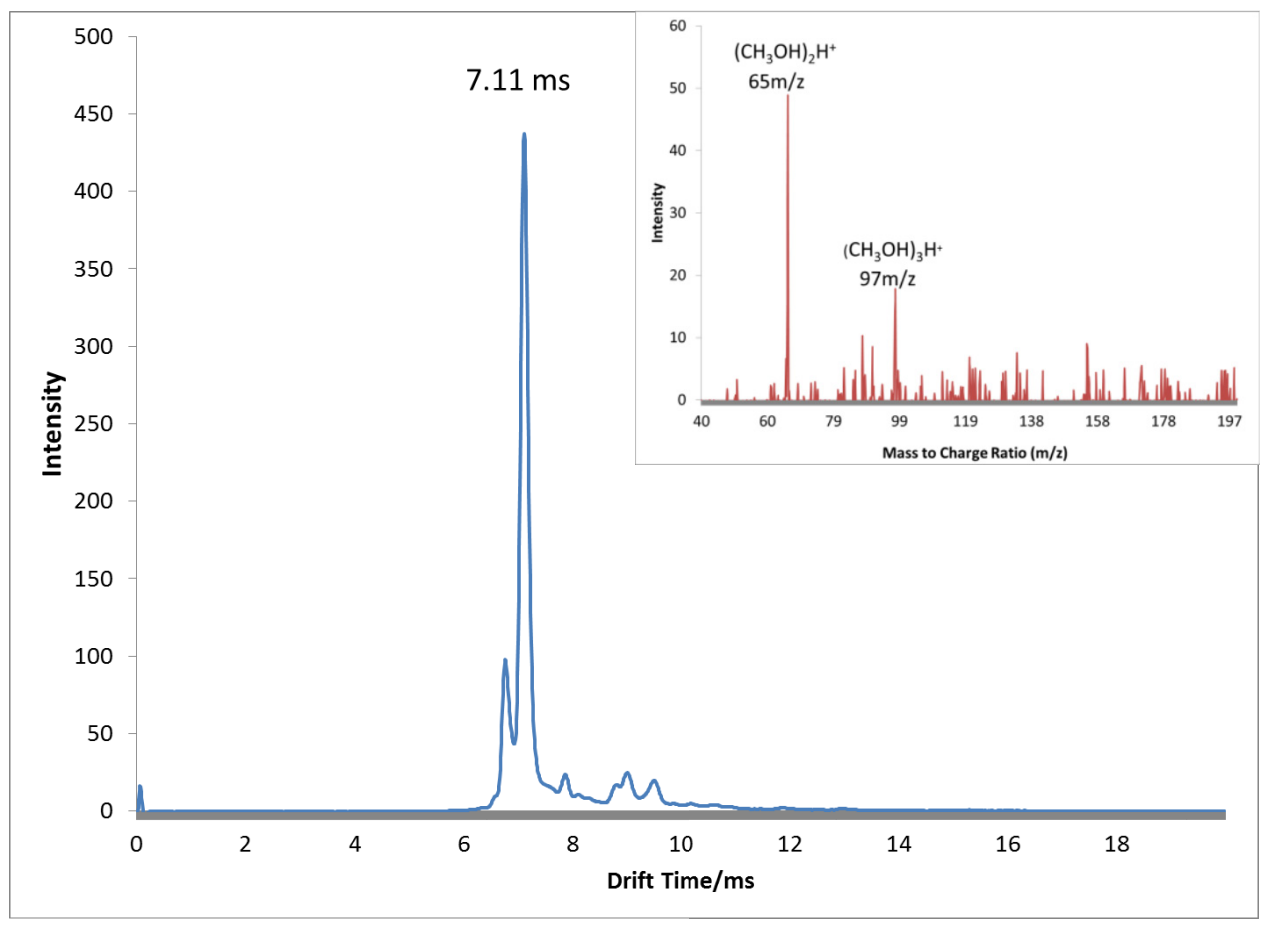

Figure 62: Species observed from ESI-IMS-MS of methanol:water mixture with $2.5 \%$ formic acid showing Ion Mobility and Mass Spectrum in Positive Ion Mode

To further enhance the ionization of some analytes, ionic salts were added to the electrospray solvent. With the addition of ammonium nitrate to the methanol:water solution, different clusters were observed as shown in Figure 63. Ions at 82, 114 and 146 $\mathrm{m} / \mathrm{z}$ were observed that corresponded to clusters of Ammonium and Methanol of formula $\left(\mathrm{CH}_{3} \mathrm{OH}\right)_{2} \mathrm{NH}_{4}{ }^{+},\left(\mathrm{CH}_{3} \mathrm{OH}\right)_{3} \mathrm{NH}_{4}{ }^{+}$and $\left(\mathrm{CH}_{3} \mathrm{OH}\right)_{4} \mathrm{NH}_{4}{ }^{+}$respectively. With the same ammonium solution ionized in the negative mode, different ions of 62 and $125 \mathrm{~m} / \mathrm{z}$ were observed as a result of clusters formed from the nitrate ions of the ammonium based salt. These corresponded to $\left(\mathrm{NO}_{3}\right)^{-}$and $\left(\mathrm{HNO}_{3}\right) \mathrm{NO}_{3}{ }^{-}$respectively (Figure 66). These ion clusters in turn transfer charge to neutral analytes within the ESI solution via protonation or adducting of positive species in the positive mode, or via de-protonation or adducting 
of a negative species in the negative mode. The background ion or solvent peak hence determined the type of analyte ion species produced for ESI-IMS analysis.

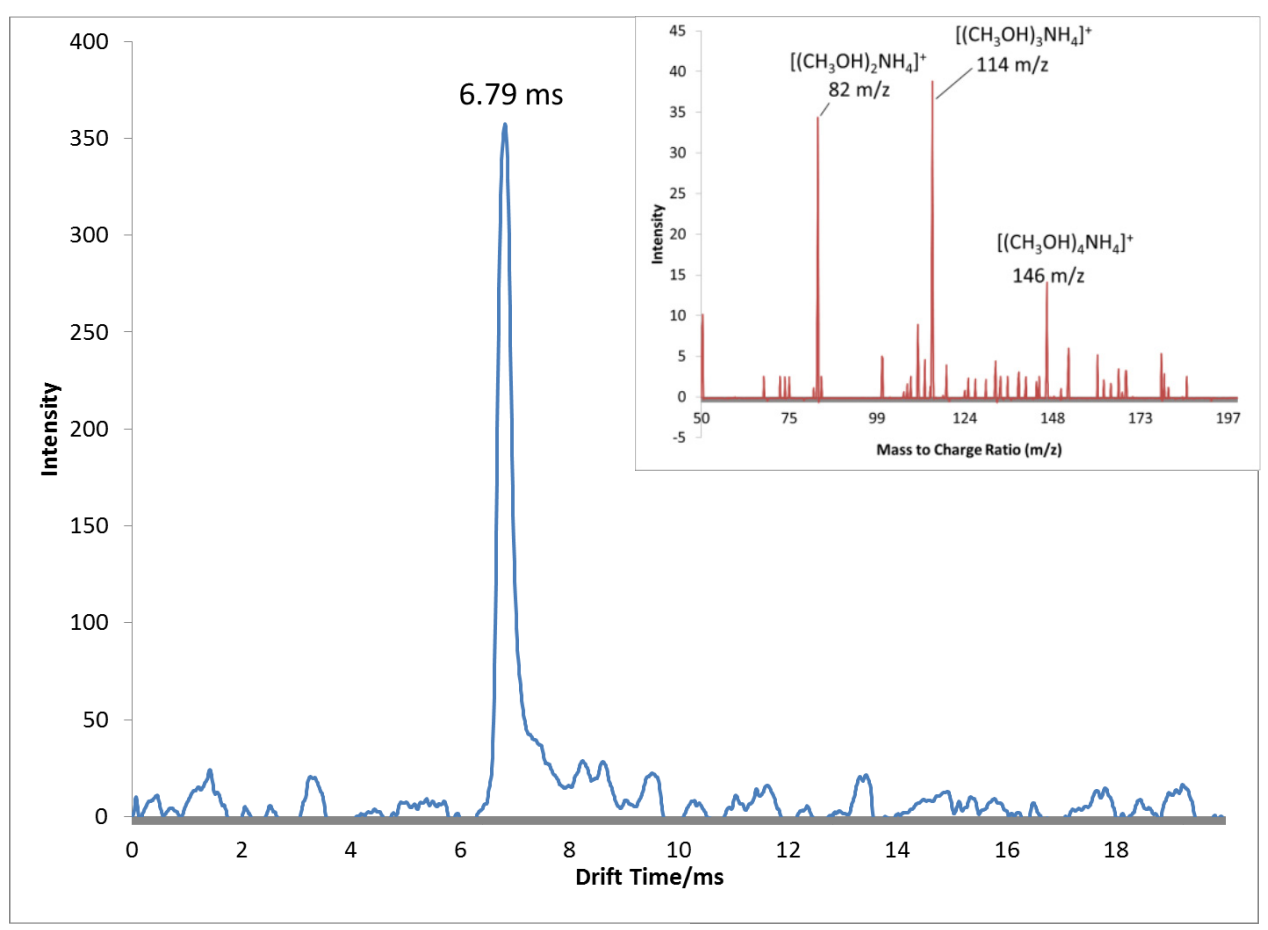

Figure 63: Species observed from ESI-IMS-MS of methanol:water with $10 \mathrm{mMol}$ ammonium nitrate and 2.5\% formic acid showing Ion Mobility and Mass Spectrum in Positive Ion Mode

Positive mode analyses, provided much simpler spectra than its negative mode counterpart, as most analytes easily underwent protonation to form a $[\mathrm{M}+\mathrm{H}]^{+}$species. As previously described, the addition of acids to the solvent system allowed for simultaneous ionization of analytes with differing proton affinities (Figure 60). A wide array of drugs were thus easily analyzed from a single mixture utilizing ESI-IMS. In addition to controlling the type of ions formed through solvent manipulation within ESI-IMS, the dynamic range of the technique was also adjusted through manipulation of solvent chemistry. Analysis of cocaine was performed via ESI-IMS and revealed a limited linear 
dynamic range of between 10ppm and 40ppm when analyzed in a methanol:water solution containing only $0.1 \%$ formic acid (Figure 64 ).

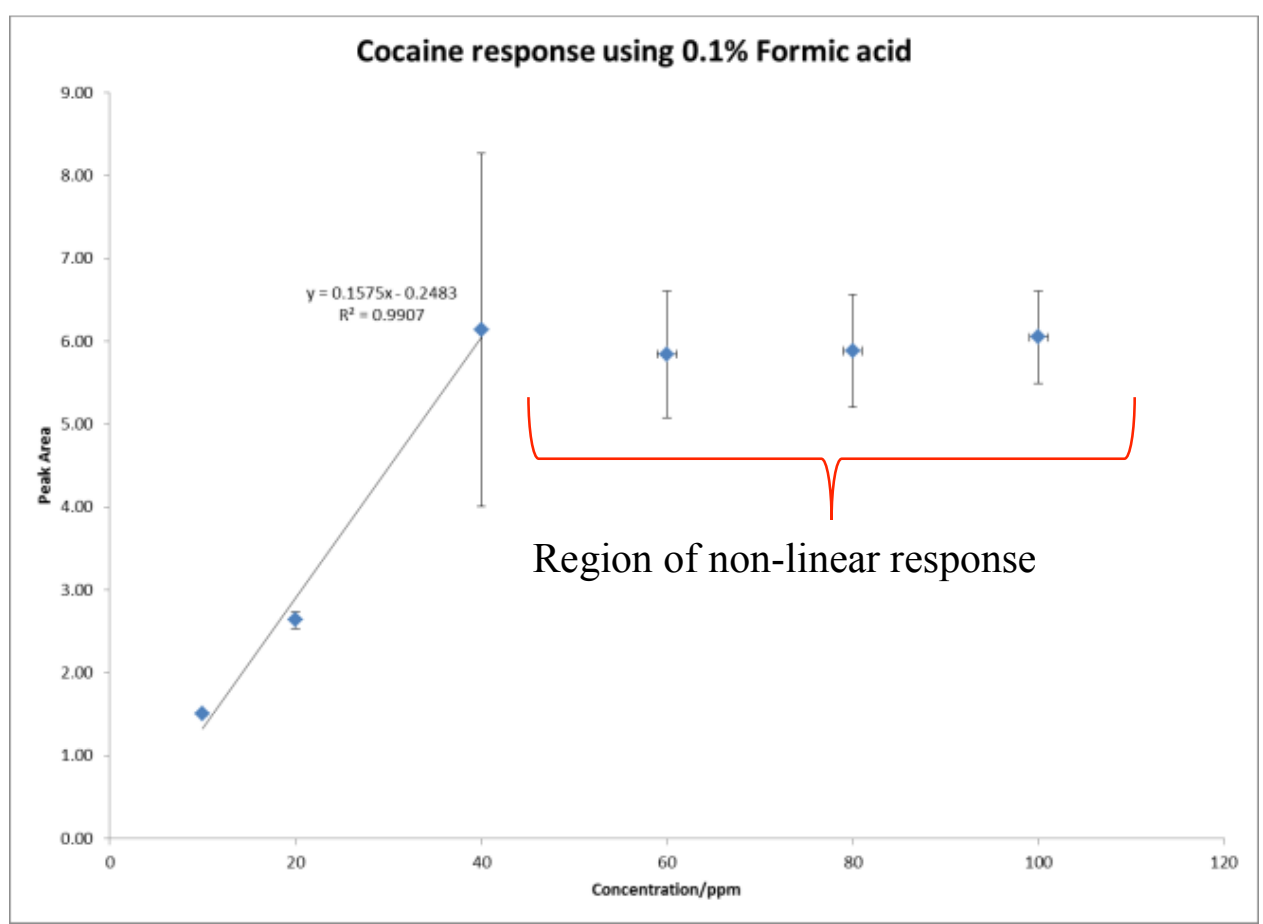

Figure 64: Response of Cocaine in $0.1 \%$ Formic acid

The signal intensity plateaus after concentrations above 40ppm. However, when this same concentration range was analyzed using higher acid strength of $5 \%$ formic acid, the dynamic range increased. Coupled with the increased dynamic range was also an improvement of the standard deviation observed from the signal intensity of each sample as shown in Figure 65. The improved signal intensity may have been attributed to improved ionization efficiency which led to more consistent ion intensity values. 


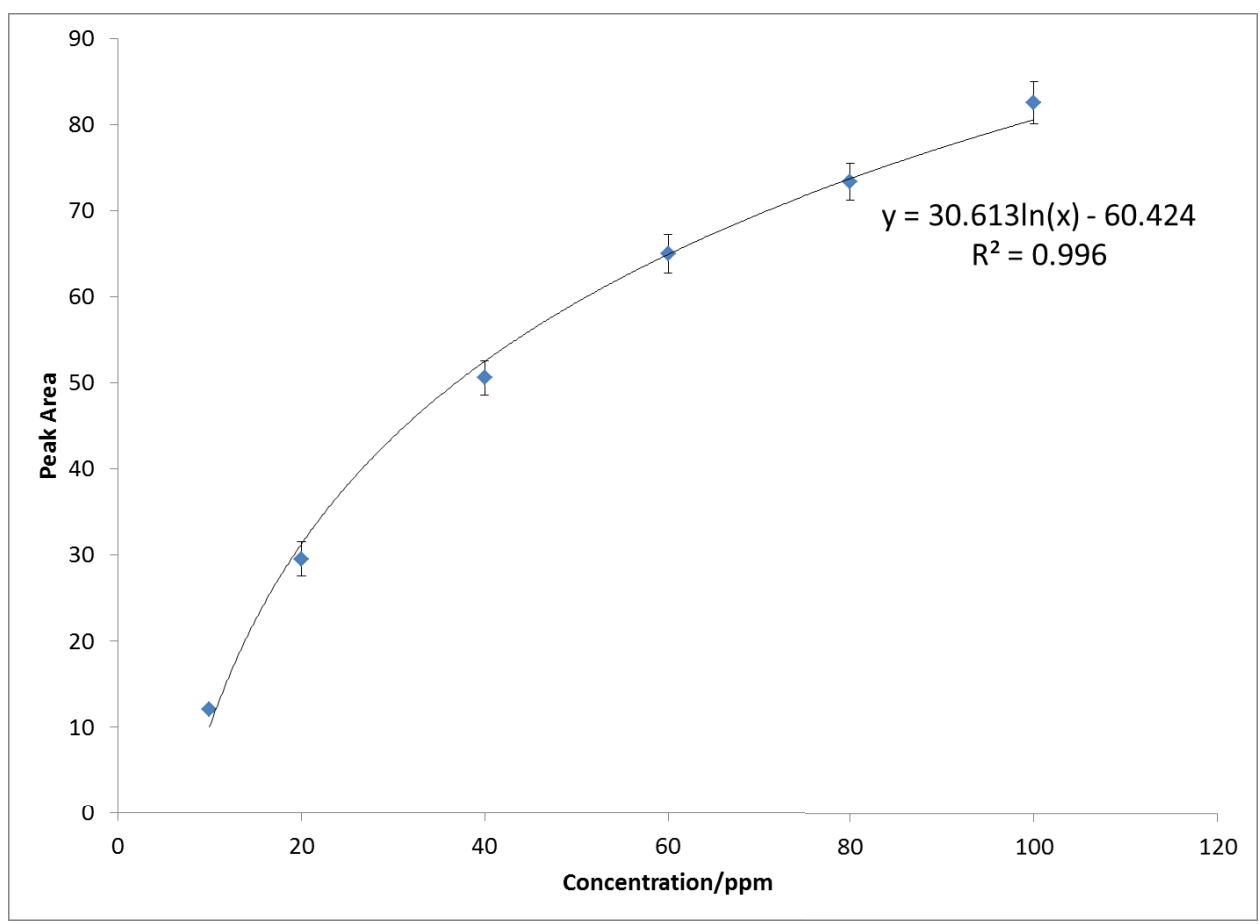

Figure 65: Response of Cocaine in methanol:water containing 5\% Formic acid

Negative mode ion chemistry is seen as more difficult than its positive mode counterpart for several reasons. First, negative mode ionization presents significant challenges as molecules that lack acidic sites on which de-protonation may occur produces low responses and detection capabilities [112]. Second, there is a greater tendency for corona discharge to occur during negative mode operation resulting in less stable spray conditions $[81 ; 113]$. Several explosive compounds where used to conduct negative mode analysis. These included nitro-aromatics (2,4-dinitrotoluene and 2,4,6-trinitrotoluene), nitro-amines (Octahydro-1,3,5,7-tetranitro-1,3,5,7-tetrazocine and 1,3,5-trinitro-1,3,5triazacyclohexane) commonly known as HMX and RDX respectively as well as nitrated aromatic amines (2-nitro-N-diphenylamine and 4-nitro-N-diphenylamine). Again the solvent system was manipulated to demonstrate the changes observed of the resulting analyte ion species and the BIP created Figure 66. 


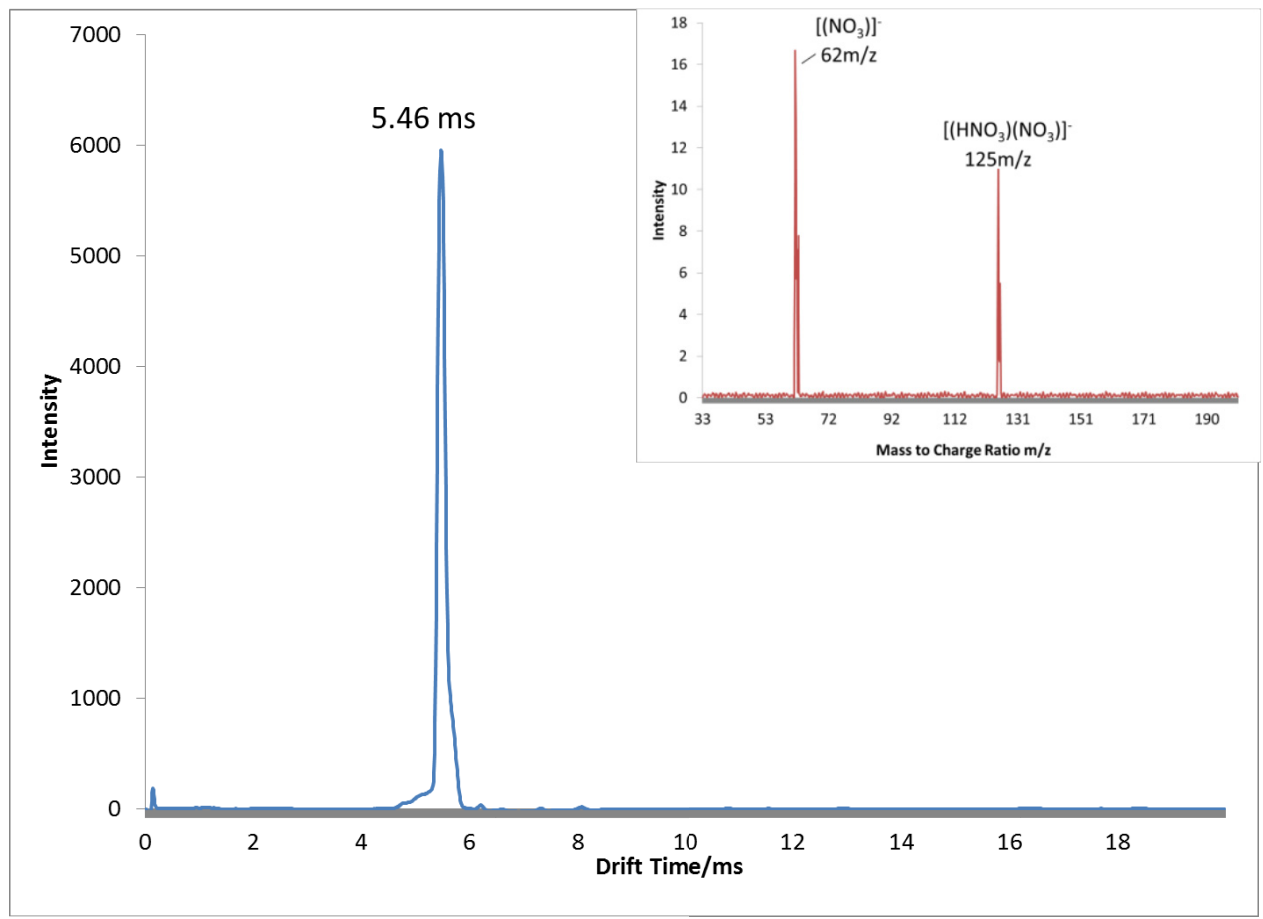

Figure 66: Species observed from ESI-IMS-MS of methanol:water with $10 \mathrm{mMol}$ ammonium nitrate showing Ion Mobility and Mass Spectrum in Negative Ion Mode

For analytes that had inherently low ionization efficiencies, adducts were more readily formed. The explosive compound 1,3,5-trinitro-1,3,5-triazacyclohexane (RDX) is one such example, with an inability to form stable negative ions on its own, RDX preferentially formed adducts determined by the solution in which it was ionized. Figure 67 shows mass spectra of the different species of RDX ions formed on the basis of the type of solutions in which it had been prepared and sprayed via ESI-IMS-MS. Three different solutions were used for these experiments, MeOH:Water, $\mathrm{MeOH}$ :Water with 10mMol of Ammonium Nitrate and $\mathrm{MeOH}$ :Water with 10mMol of Sodium Chloride. Each was used to make a 100ppm solution of RDX which was then analyzed via ESI- 
IMS-MS. The mass spectra obtained differed for each solvent system as shown in Figure 67.

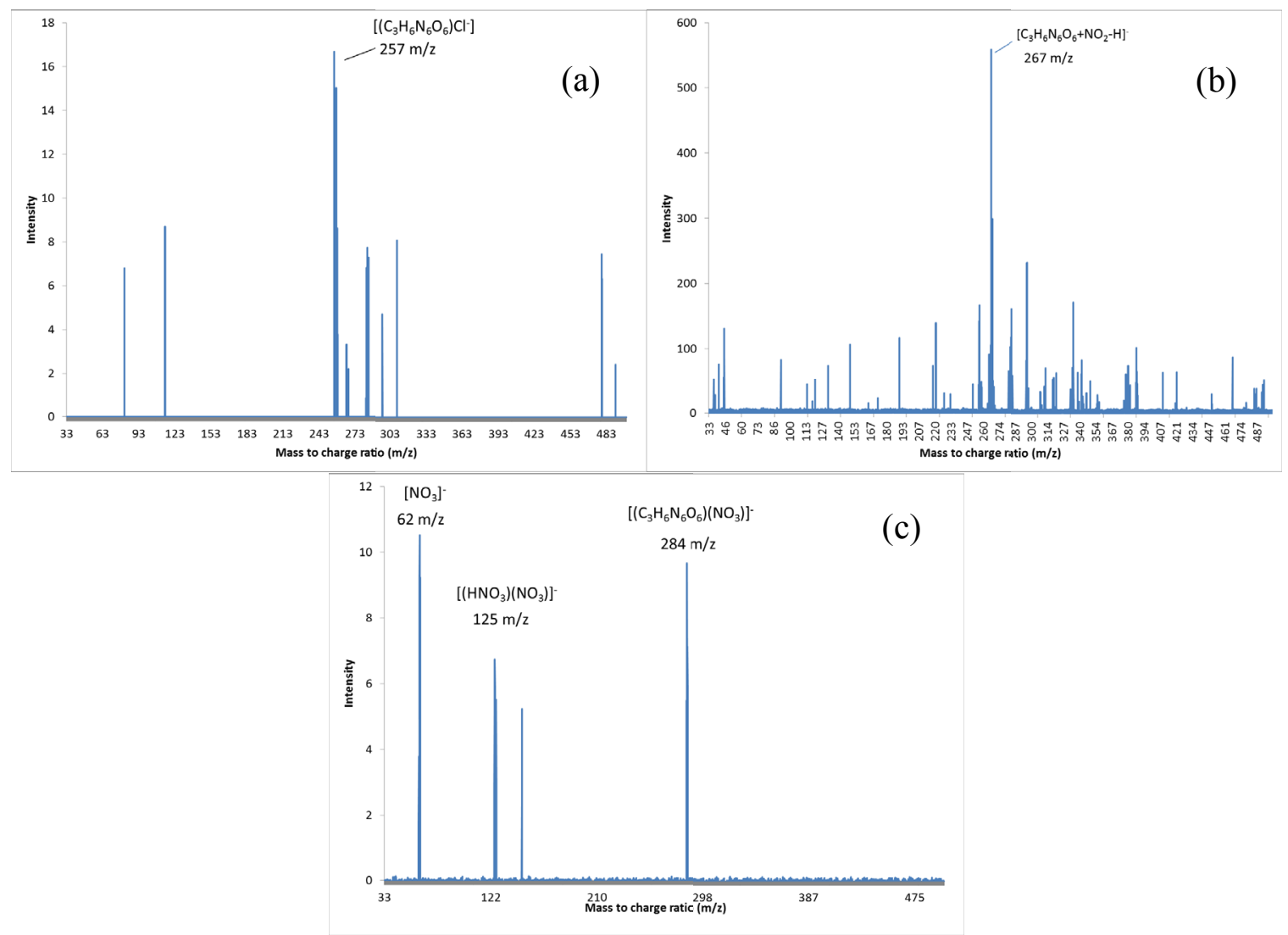

Figure 67: Mass Spectra of RDX species formed in (a) sodium chloride based solvent, (b) methanol:water only and (c) ammonium nitrate based solvent

Of interest was the detection of RDX in Figure $67 \mathrm{~b}$ where no adducting agents are present within the electrospray solvent. The dominant ion at $\mathrm{m} / \mathrm{z} 267$ has been reported as the molecular formula [M+NO2-H]- for RDX in methanol:water solutions [32], however, this structure has not been confirmed. In these experiments, none of the proton abstracted $[\mathrm{M}-\mathrm{H}]-$ ions were detected for these nitro-amine derived explosives as opposed to nitroaromatics such as 2,4,6-trinitrotoluene (TNT) and 2,4-dinitrotoluene (DNT) that readily formed proton abstracted ionic species. When spraying nitro-aromatic explosives such as 
TNT and DNT using an electrospray solvent containing nitrate anions (Ammonium nitrate solution), a complete suppression of these nitro-aromatics was observed as opposed to an increase in signal intensity which was observed for nitro-amines such as RDX. Previous reports into the proton abstraction process that allows negative ionization of analytes at atmospheric pressure, reveals that the reactant ion (charged solvent) and analyte interact so that a new ion is formed as well as a neutral molecule (Equation 14). If the ESI solvent produces a reactant ion that is unable to accept a proton from the neutral analyte, then ion suppression occurs as observed with nitro-aromatics DNT and TNT when sprayed with nitrate salts such as ammonium nitrate [3]. But, if the analyte is able to undergo ion attachment (Equation 15) as is the case with nitro-amines in the presence of nitrate salts, then ionization occurs while in this solution (Figure 67). As a result, a mixture of nitro-aromatic and nitro-amines sprayed with a nitrate salt based solution, will only show ionization of the nitro-amines owing to the difference in proton affinity between the nitro-aromatics and the nitrate ions formed during ESI processes [114]. As a result of these findings, an ESI solution that allows the simultaneous ionization of a variety of analytes in the negative mode, cannot include nitrate salts, as these would suppress any nitro-aromatics within the solution. This ionization suppression however is not the case when chloride ions are used in the ESI solvent. No such suppression was observed with nitro-aromatics and solvents containing chloride ions. During the course of these experiments, no adducted species were formed with the nitro-aromatics DNT and TNT with any solution, these species only formed proton abstracted ionic species $[\mathrm{M}-\mathrm{H}]-$.

Equation 14 Proton abstraction in negative mode ESI [115]

$$
A^{-}+M \rightarrow A H+[M-H]^{-}
$$




\section{Equation 15 Anion attachment in negative mode ESI [115]$$
A H^{-}+M \rightarrow M A H^{-}
$$

The ability to utilize chloride ions to form stable analyte ions within a mixture was achieved by dissolving $10 \mathrm{mMol}$ of sodium chloride within a methanol:water solution. However, this proved problematic with extended use, as nonvolatile deposits would form on high voltage components within the ion mobility spectrometer drift tube. Addition of sodium chloride led to arcing and damage to some electrical components of the IMS system. An alternative source of chloride ions was found in the form of organic methyl halides such as chloroform. These organic halides performed equally to the inorganic sodium chloride additive, allowing for the formation of chloride adducted species for nitro-amines, while not suppressing the ionization of nitro-aromatics and had no deposits that damaged the IMS. Analysis of some analytes utilizing this chloroform modified solution are displayed in Figure 68, the simultaneous formation of both the proton abstracted and chlorine adducted species may be seen for nitrated aromatic amine explosive compounds such as 4-nitro-Diphenylamine (Figure 68b). 


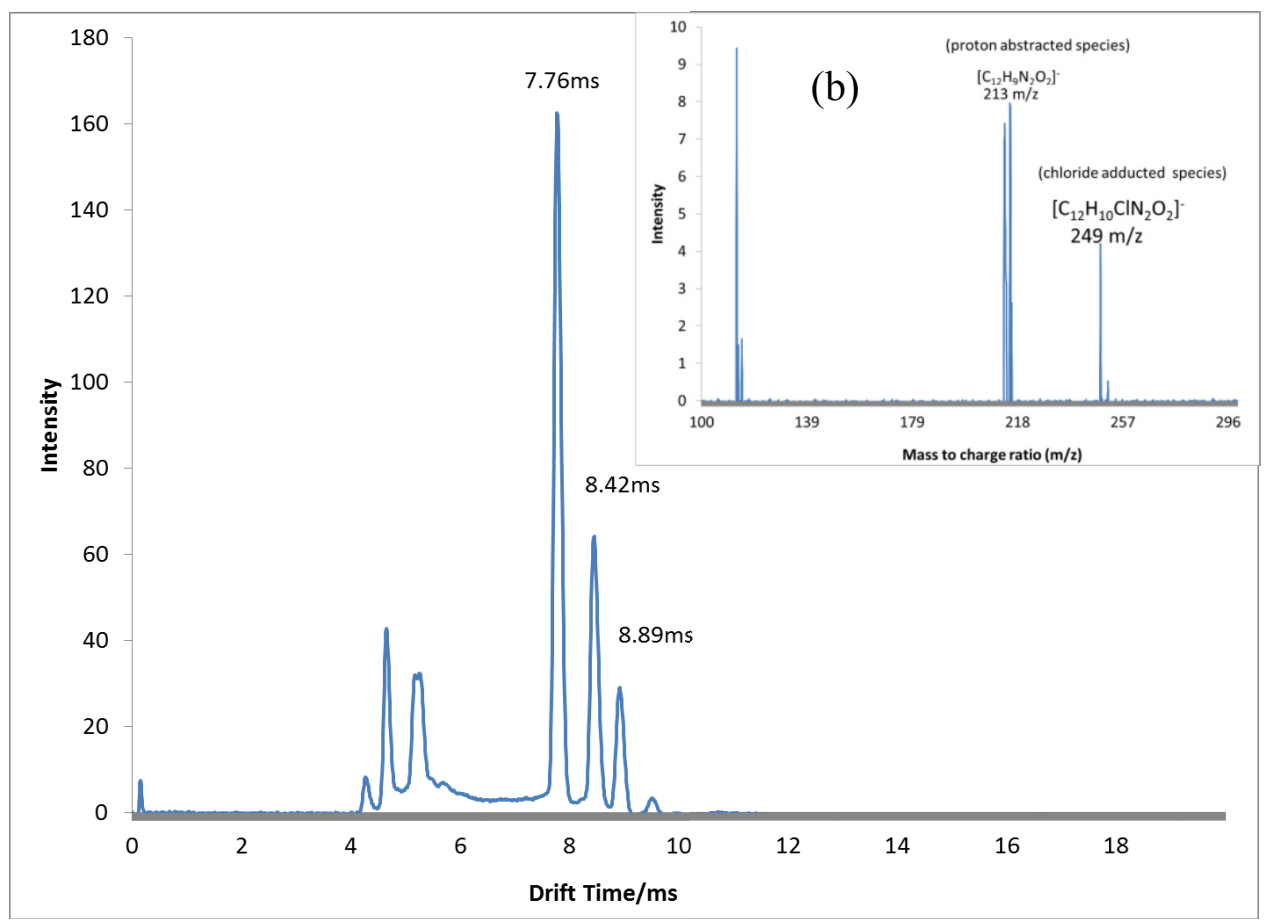

Figure 68: Species observed from ESI-IMS-MS of 4-Nitro-diphenylamine dissolved in methanol:water with $0.1 \%$ chloroform by (a) Ion mobility in negative ion mode and (b) Full scan mass spectrum in negative ion mode

Multiple peaks are observed for this single analyte within the ion mobility spectrometer corresponding to both the proton abstracted $[\mathrm{M}-\mathrm{H}]^{-}$at $7.8 \mathrm{~ms}$ and chloride adducted species $[\mathrm{M}+\mathrm{CL}]^{-}$at $8.5 \mathrm{~ms}$. A third peak at $8.9 \mathrm{~ms}$ was observed but the corresponding mass to charge was not detected by the mass spectrometer. Analysis of this compound without the addition of chlorine in electrospray solution only produced the proton abstracted species observed at $7.8 \mathrm{~ms}$. With regards to the effect that solvent chemistry plays on altering signal intensity, a similar trend to that observed in positive mode analysis through the addition of acid modifiers was also observed in the negative mode. By utilizing chlorinated solvents instead of acids, increased signal response and improved reproducibility was observed during negative mode analysis. Figure 69 shows the results 
when 2,4 dinitrotoluene was analyzed using a solvent mixture with chloroform and one without. The solvent system containing chloroform produced more intense and more consistent ion signals of the analyte.

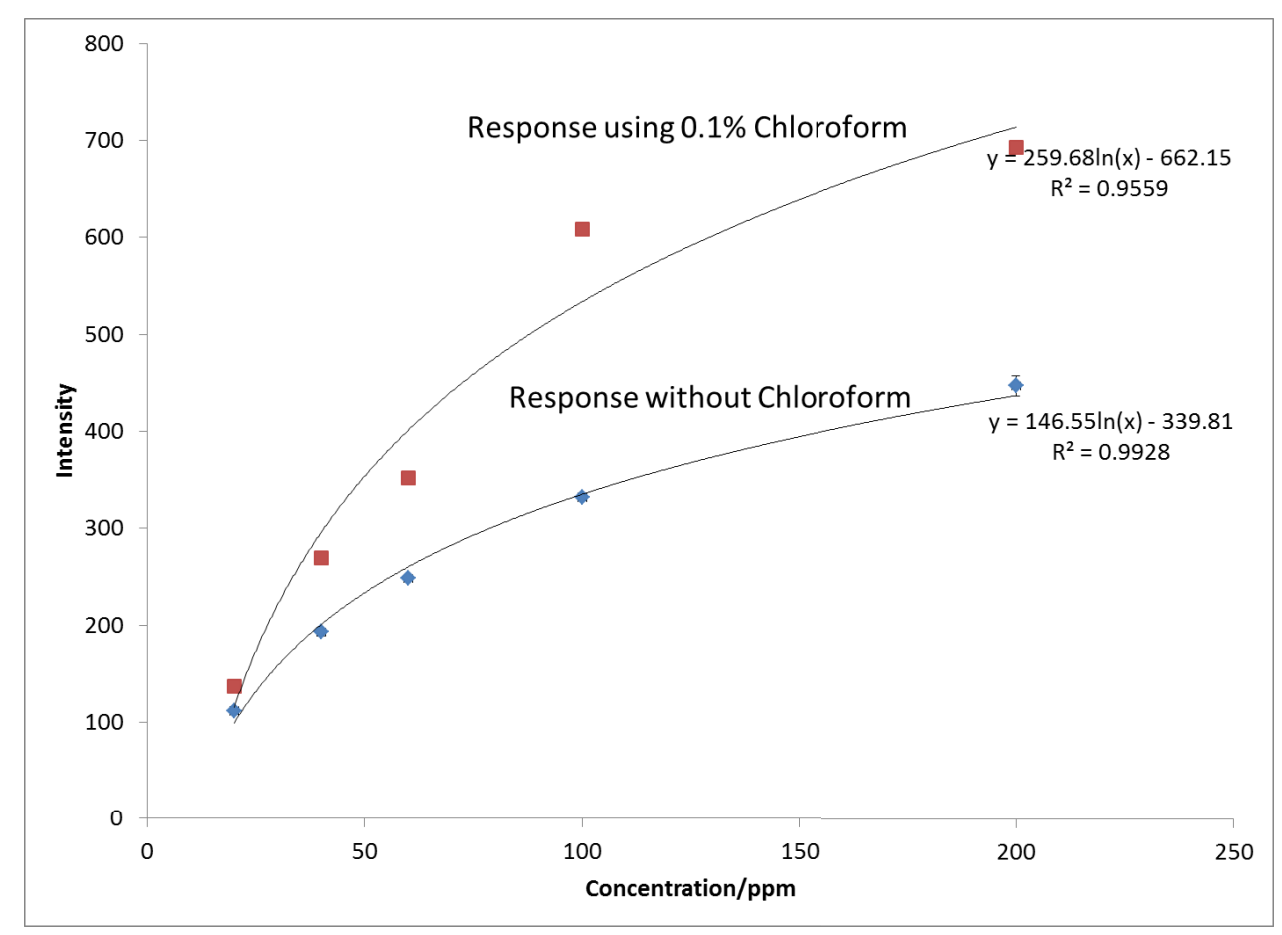

Figure 69: Effect of chloroform on ion intensity for negative mode analysis showing enhanced detection of 2,4 Dinitrotoluene

The use of the methyl halides for negative mode analysis and the use of acid modifiers for positive mode analysis for ESI-IMS-MS analysis therefore proved instrumental in providing more intense and consistent results. For negative mode analysis in particular, it was found that some modifiers such as nitrate salts, enhanced analytes such as nitroamines while this same modifier suppressed other analytes such as nitro-aromatics. Modifiers such as chlorides from methyl halides were most effective for negative mode multiplexing analysis at levels of $0.1 \% \mathrm{v} / \mathrm{v}$ while acid modifiers such as formic acid were most effective in positive mode analysis for multiplexing at levels of $5 \% \mathrm{v} / \mathrm{v}$. 


\subsubsection{Conclusions:}

The ability to alter ion response by changing solution chemistry has allowed for a better understanding of gas phase reactions that occur under atmospheric conditions. These findings were accepted by peer review and have since been published [116]. Rapid and sensitive analysis is now possible of more complicated mixtures than were previously analyzed using traditional ${ }^{63} \mathrm{Ni}$-based IMS devices. In all cases for positive mode detection, simple protonated species were observed for analytes, producing an $[\mathrm{M}+\mathrm{H}]^{+}$ ion, irrespective of the solution chemistry of the electrospray solvent. For negative mode analysis, a wider range of analyte ion species were observed that were dependent on the solution chemistry being sprayed as was observed for nitro-amines such as RDX and nitrated aromatic amine 4-N-DPA. Changes in solvent chemistry were able to produce changes in the analyte ion species being formed in the negative mode[84]. Though electrospray ionization is heavily dependent on concentration which has been reported to provide better sensitivity for low volume samples [78], the addition of acid modifiers in the positive mode and methyl halides in the negative mode, proved useful in allowing simultaneous ionization of analytes and increasing overall signal intensity of analytes. 
4.3 Application of Appropriate solvent chemistry for the Chiral analysis of Amphetamine Type Substances

With the optimal solvent system determined for analysis of either single or multiple component mixtures, focus was placed on applying this solvent system to the analysis of chiral amphetamine type substances. With the use of the solvents containing sufficient acid strength $(2.5 \%$ for all experiments $)$, it was possible to analyze multicomponent mixtures on the RA4100 (see Figure 70). Of recurring interest is the lack of a BIP for the solutions analyzed in low acid solutions, the lack of a BIP was observed as a tell-tale sign during analysis that the analytes were depleting all available ions present within the solvent system and indicated that there may be preferential ionization resulting from charge competition occurring. Figure 70 also highlights the lack of response of some analytes when solutions containing low acid concentrations are used, again allowing the possibility of not identifying analytes when they are actually present from real case work samples. The use of this solvent system therefore reduces these errors within the crime lab when this technique is utilized. 


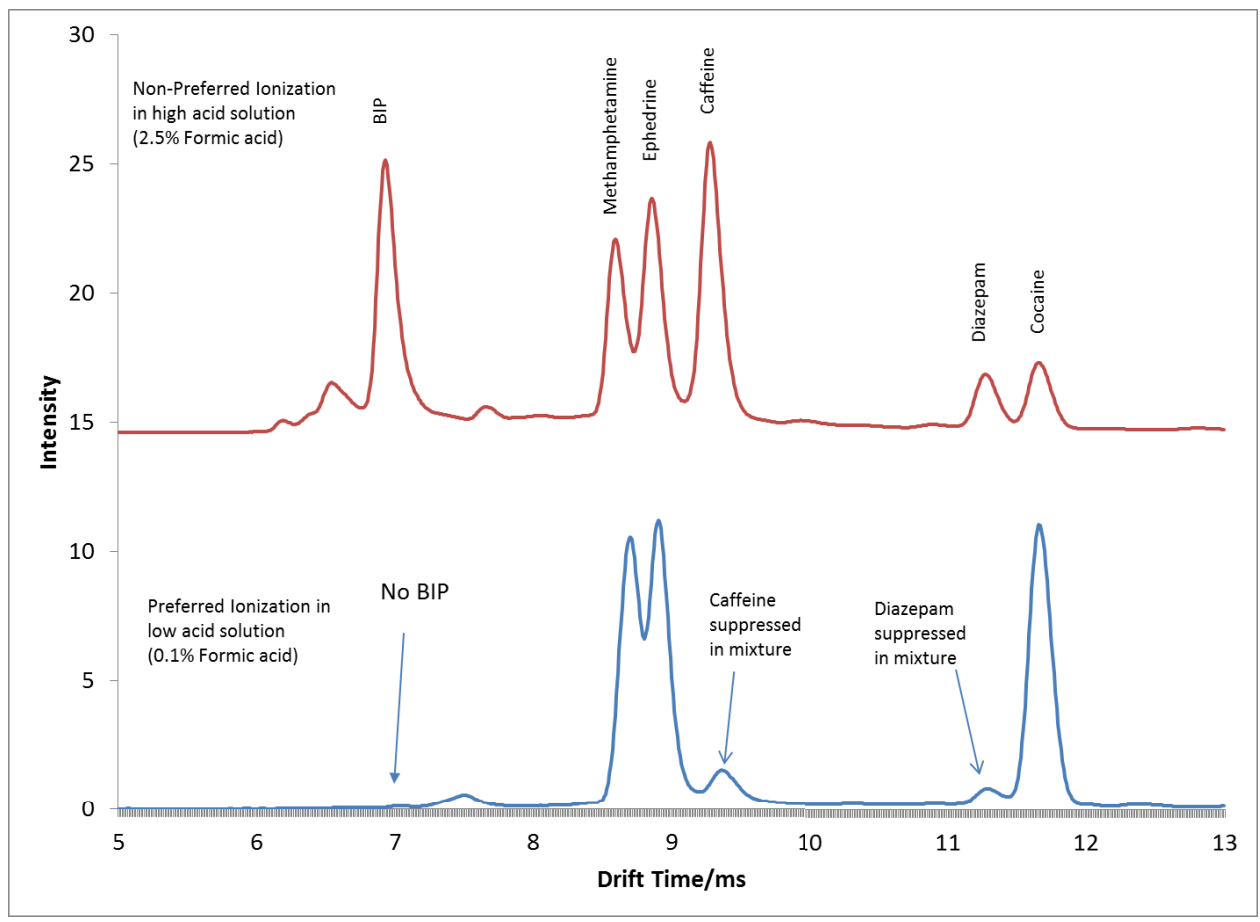

Figure 70: Analysis of 50ppm Caffeine, R-Methamphetamine, SR-Ephedrine, Diazepam and Cocaine on RA4100 using both $2.5 \%$ formic acid and $0.1 \%$ formic acid

As the RA4100 is also able to provide mass spectral data for samples analyzed, the same multicomponent mixture of caffeine, methamphetamine, ephedrine, diazepam and cocaine was shown to produce their characteristic protonated molecular ions when a multicomponent mixture was analyzed (Figure 71). 


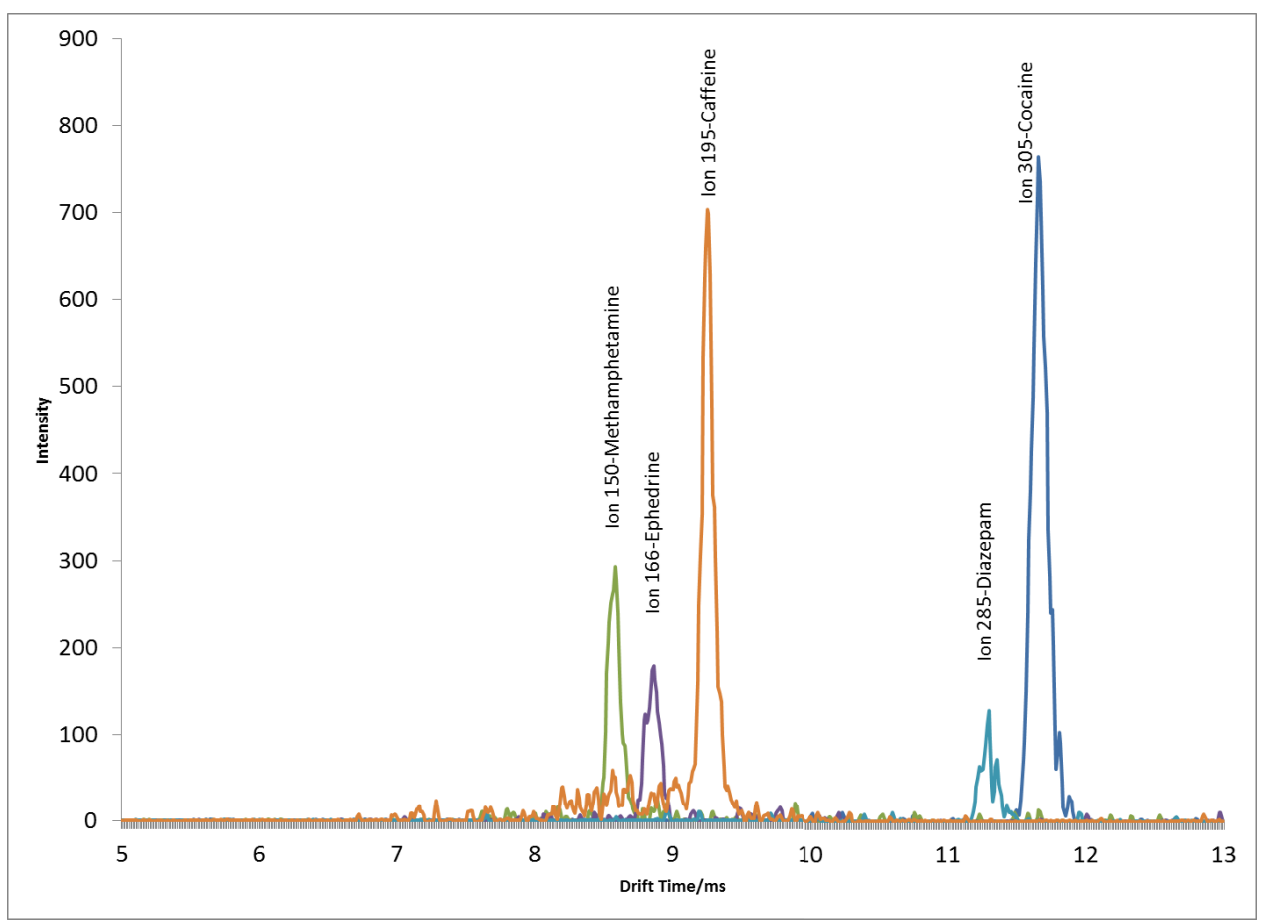

Figure 71: Single Ion monitoring plot of molecular ions for a multicomponent mixture

With the parameters required to effectively analyze mixtures utilizing the RA4100 determined, focus was shifted to improving the separation of the target amphetamine type substances. These substances were analyzed on the RA4100 to determine their drift times and type of ionic species formed (Table 6). 
Table 6: Observed drift times and reduced mobility of target analytes

\begin{tabular}{|l|c|c|c|}
\hline Compound & $\begin{array}{l}\text { Ion } \\
\text { Monitored } \\
\text { (RMM) }\end{array}$ & $\begin{array}{l}\text { Drift } \\
\text { Time/ms }\end{array}$ & $\begin{array}{l}\text { Reduced } \\
\text { Mobility }\end{array}$ \\
\hline R-Methamphetamine & 150 & 8.694 & 1.64 \\
\hline S-Methamphetamine & 150 & 8.696 & 1.64 \\
\hline SR Ephedrine & 166 & 9.007 & 1.59 \\
\hline RS Ephedrine & 166 & 9.012 & 1.58 \\
\hline RR-Pseudoephedrine & 166 & 9.029 & 1.58 \\
\hline SS-Pseudoephedrine & 166 & 9.031 & 1.58 \\
\hline
\end{tabular}

On the basis of the drift times observed, the reduced mobility values for each analyte was calculated by comparison to a standard peak of 2,4 Lutidine $\left(\mathrm{K}_{0} 1.95 \mathrm{~cm}^{2} \mathrm{~V}^{-1}[88]\right)$ as described in Equation 10. The spectra of compounds analyzed revealed unresolved peaks between SR and RS ephedrine and RR and SS pseudoephedrine as seen in Figure 72.

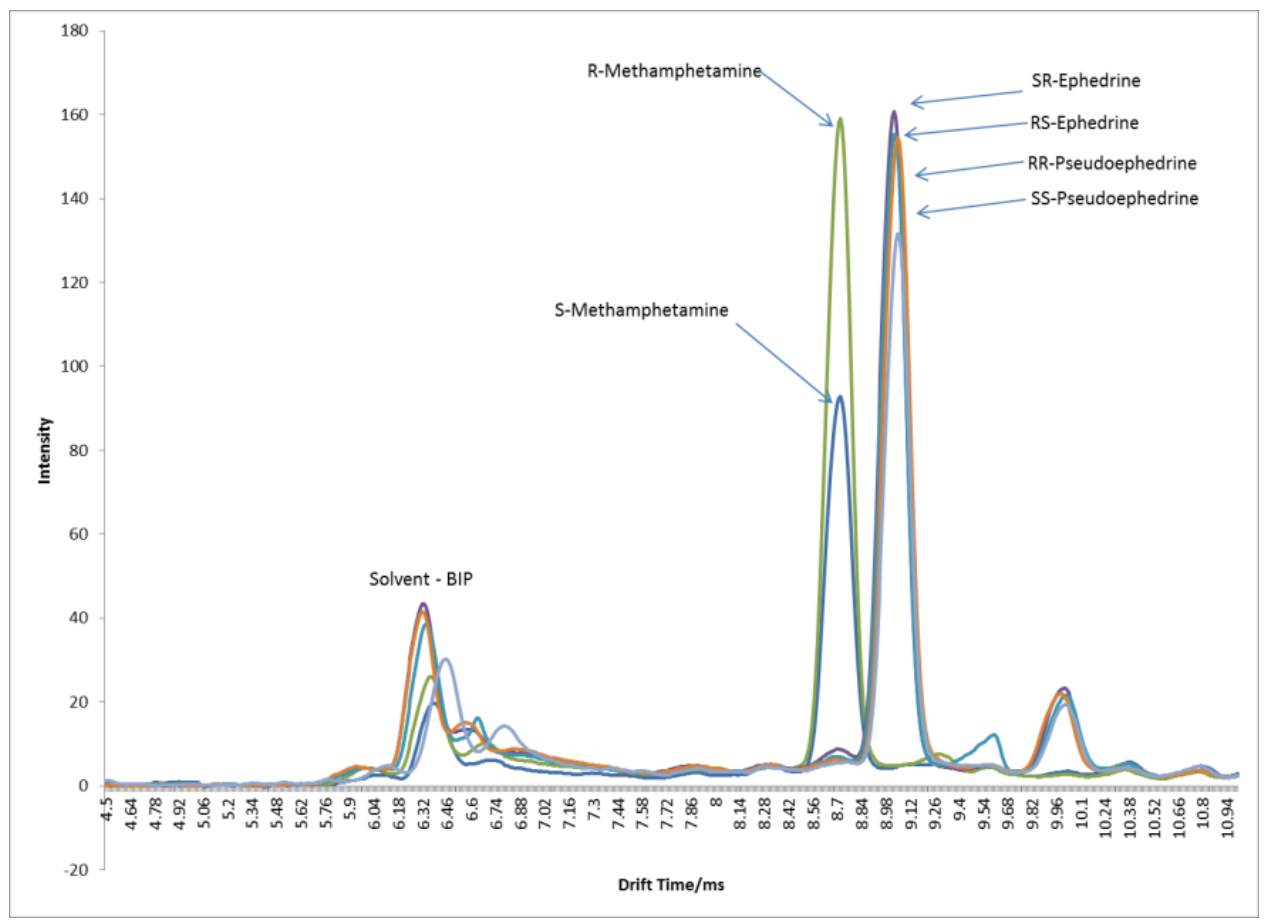

Figure 72: Overlay of target analytes on ESI-IMS-MS (RA4100)

The observed overlap follows the trend observed for the same compounds analyzed on the OTS Ionscan 400B previously shown in Figure 33. Attempts to separate overlapping 
compounds were continued from the experiments utilizing the Ionscan 400B with the introduction of modifier into the drift tube. Recalling from those experiments that the 400B suffered from inefficient modifier introduction which resulted in a non-linear increase between the amount of modifier introduced into the drift tube and the amount of separation observed between chiral compounds (see Figure 41). To overcome this, the RA4100 is equipped with an independently heated modifier inlet with a dedicated gas flow to ensure that the modifier is vaporized and swept into the drift tube (Figure 49). The modifier inlet prevented the decrease in modifier response observed with the Ionscan 400B (Figure 41) and ensured that either small amounts of modifier were required resulting in a more efficient technique, or that much larger amounts of modifier could be introduced if required for certain analyses. The modifier itself was infused similar to the method used with the Ionscan 400B by way of a gas tight syringe and an infusion pump.

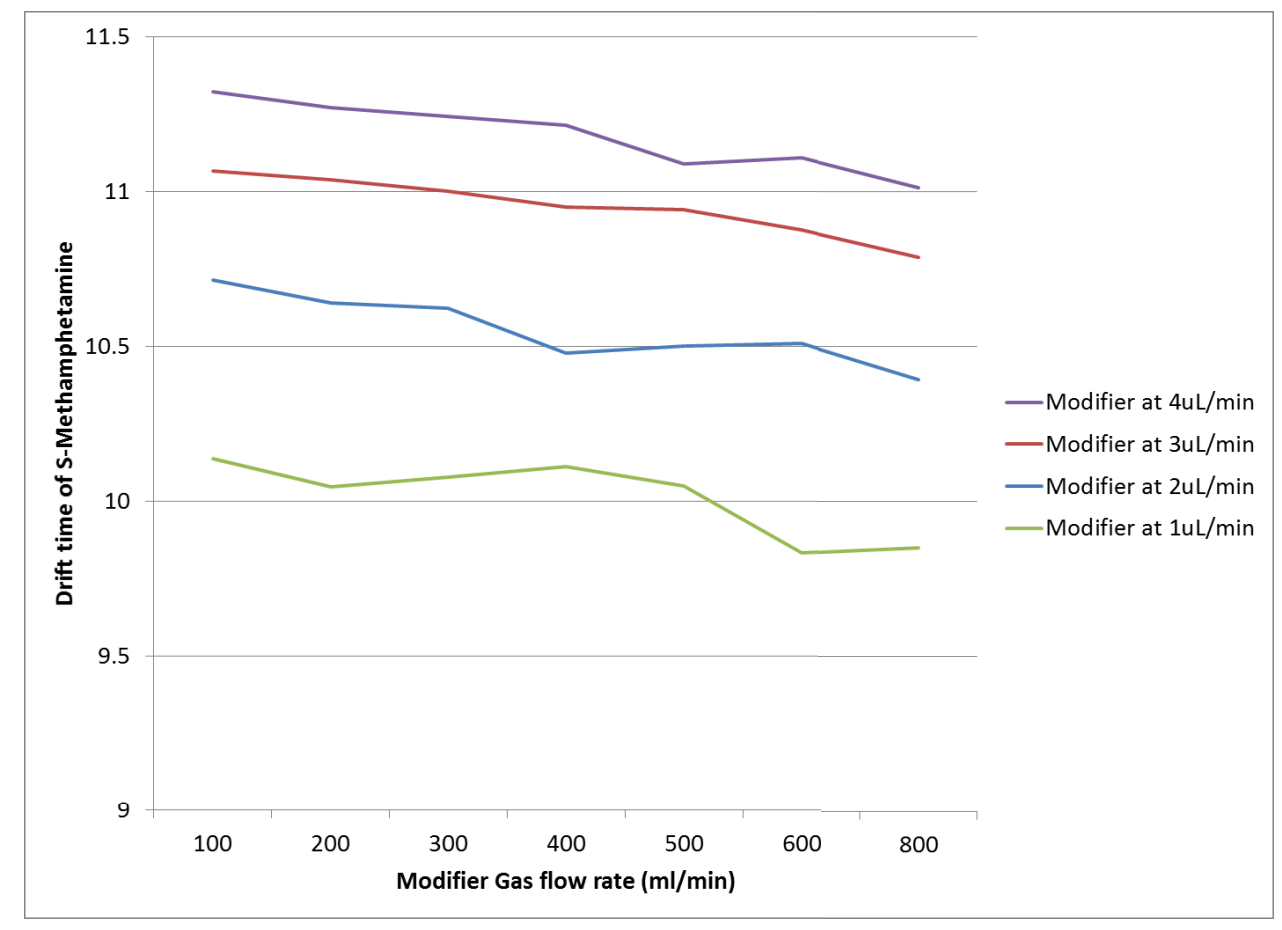

Figure 73: Effect of Modifier Infusion rate on analyte drift time 
Figure 73 reveals how the infusion of the modifier affects the drift time of an individual analyte. As the infusion rate of the modifier is increased, the drift time of the analyte increases, indicating that there is greater interaction of the modifier and analyte as more modifier is infused. A plot of drift times for target analytes of forensic interest, RSEphedrine and RR-Pseudoephedrine, versus the infusion rate of the chiral modifier, S-2butanol, revealed that the modifier can be infused to rates as high as $16 \mu \mathrm{L}$ per minute or $960 \mu \mathrm{L} /$ Hour (Figure 74). The flow was greater than the infusion rate possible using the OTS Ionscan $400 \mathrm{~B}$, that was only able to achieve $300 \mu \mathrm{L} /$ Hour with its configuration (Figure 41). Figure 74 reveals a steady increase in the drift times of the analytes with an increase in modifier infusion rates and this was seen as an indication that the modifier was able to interact with the analyte for the entire range of infusion rates used.

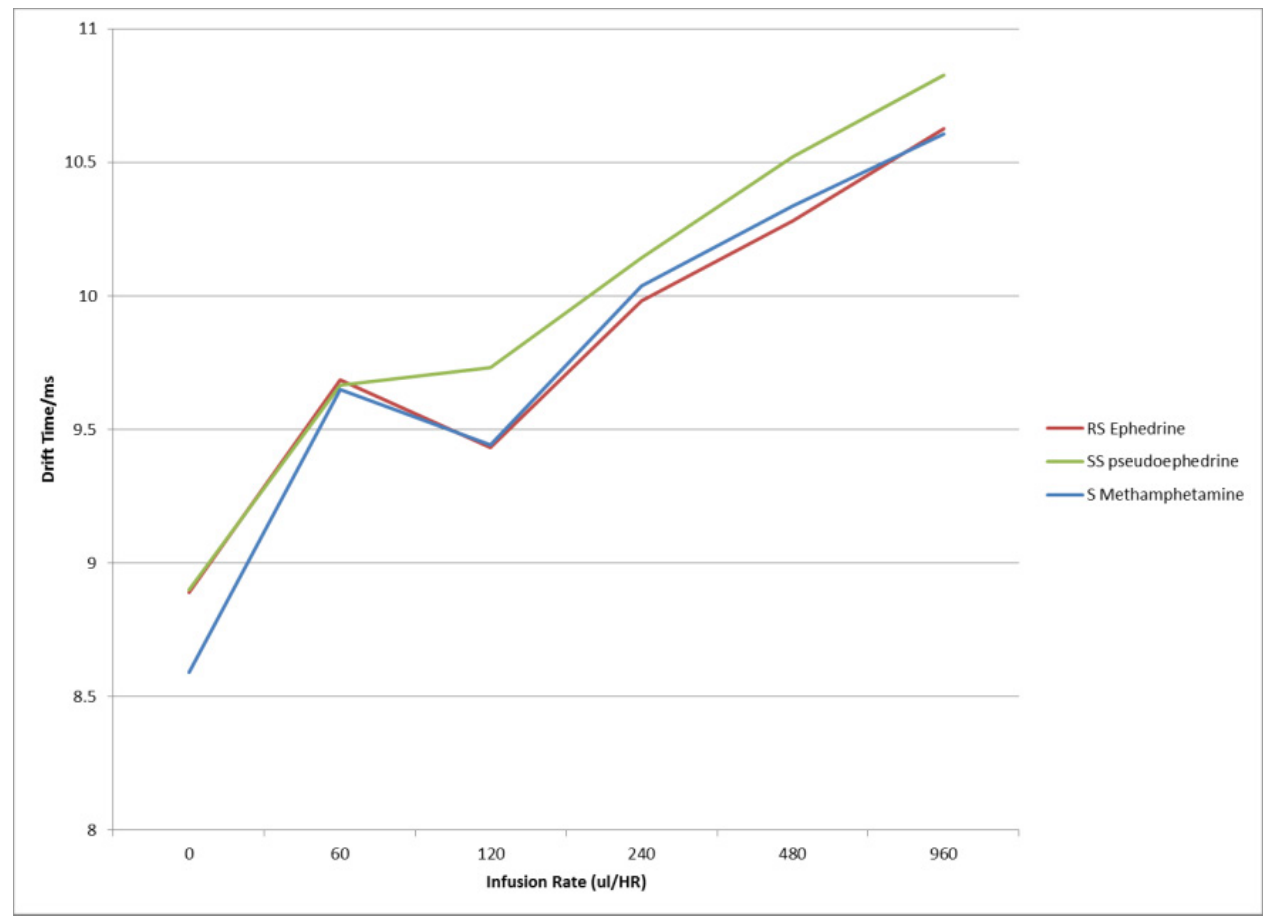

Figure 74: Effect of Modifier infusion rate on drift time separation of analytes on RA4100 
It was also found through another series of experiments, that the flow rate of the independent gas flow connected to the modifier also played a role in the efficiency of the modifier being introduced. By holding the modifier infusion rate constant at $240 \mu \mathrm{L}$ per hour or $4 \mu \mathrm{L}$ per minute, the modifier gas flow was varied and the interaction of the modifier versus analytes plotted (Figure 75).

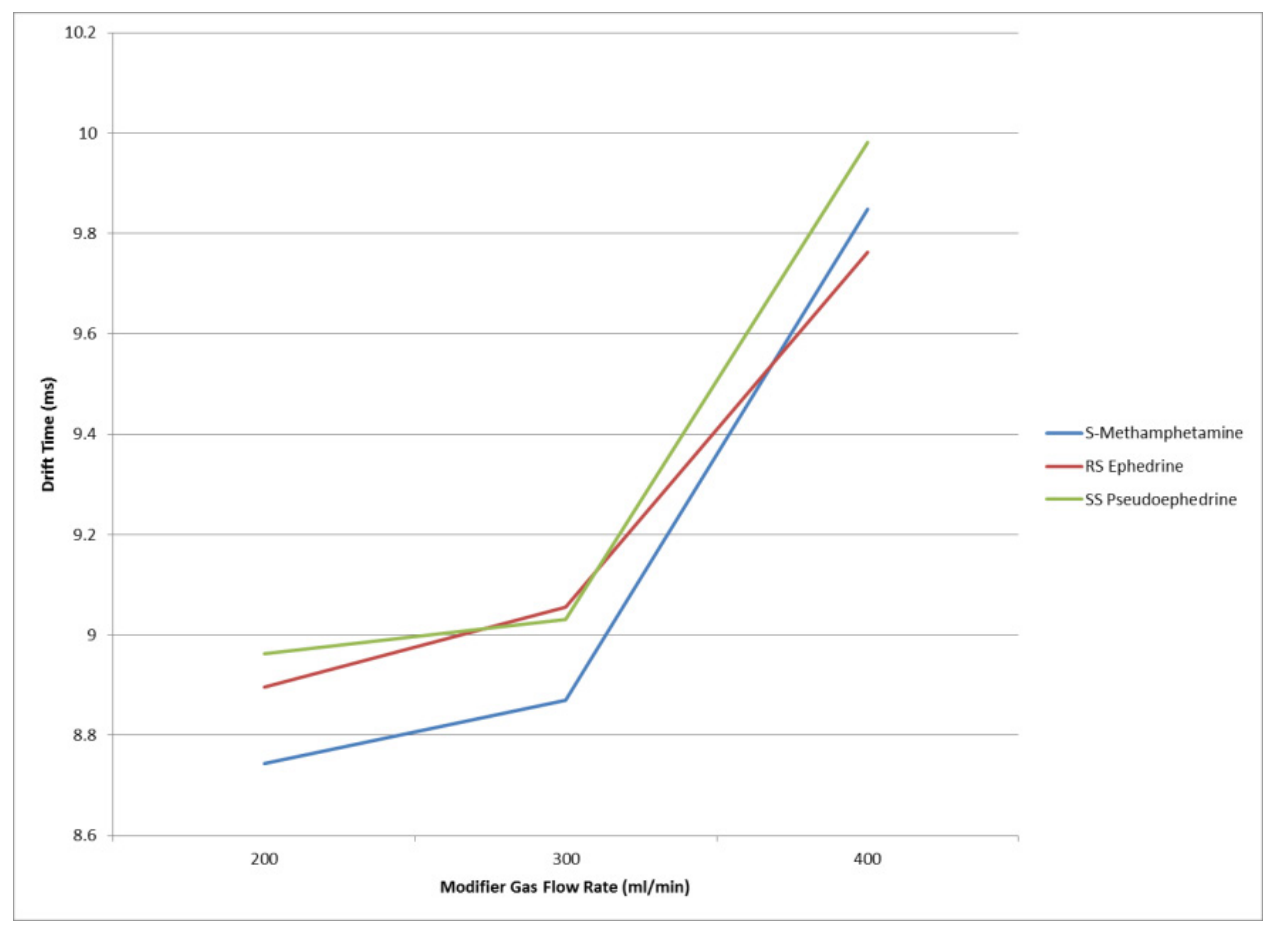

Figure 75: Effect of Modifier Gas flow on drift time of analytes

The interaction between the modifier gas flow and the drift time of the analytes was uncharacteristic as the modifier gas flow was believed to only affect the transport of the modifier into the drift tube and hence have a similar trend to that observed in Figure 74. However, the interaction observed by varying the modifier gas flow rate resulted in slightly different drift times for each analyte as opposed to an equal and systematic change across all analytes. None the less, the modifier gas flow rate was maintained at 
$400 \mathrm{ml} /$ minute in order to conserve gas usage while maintaining sufficient transport of modifier into the drift tube. As revealed in Table 6, the RA4100 is able to give the mass to charge ratio of the ions being formed by the electrospray source and then being separated by the ion mobility spectrometer. Though the electrospray source is a soft ionization source as previously explained, some fragmentation was observed for some of the analytes studied. Case in point, SS-Pseudoephedrine with a molecular mass of 165 amu and an expected protonated molecular ion of $166 \mathrm{amu}$. However, the mass spectrum of SS-Pseudoephedrine using the RA4100 produces an ion fragment of $149 \mathrm{amu}$ in addition to the 166 molecular ion (Figure 76).

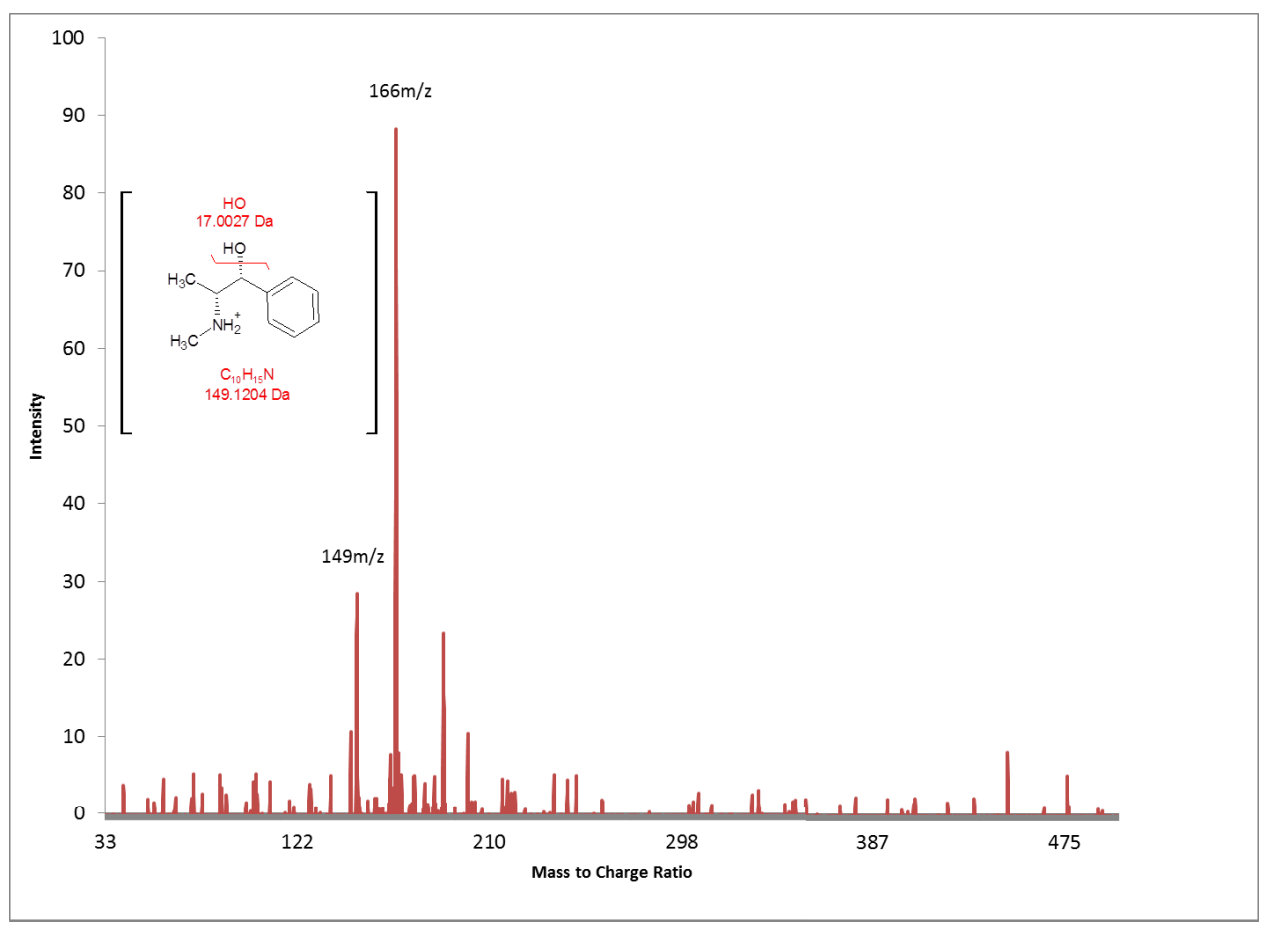

Figure 76: Mass Spectrum of SS-Pseudoephedrine on RA4100 
Both the 149 and 166 ion occur at the same drift time (9 ms) indicating that both ions originate from the same molecule. The 149 ion results from the loss of the hydroxyl group attached to the alpha carbon of the aromatic ring. Figure 77 shows the single ion monitoring of these ions from SS-Pseudoephedrine as observed on the RA4100.

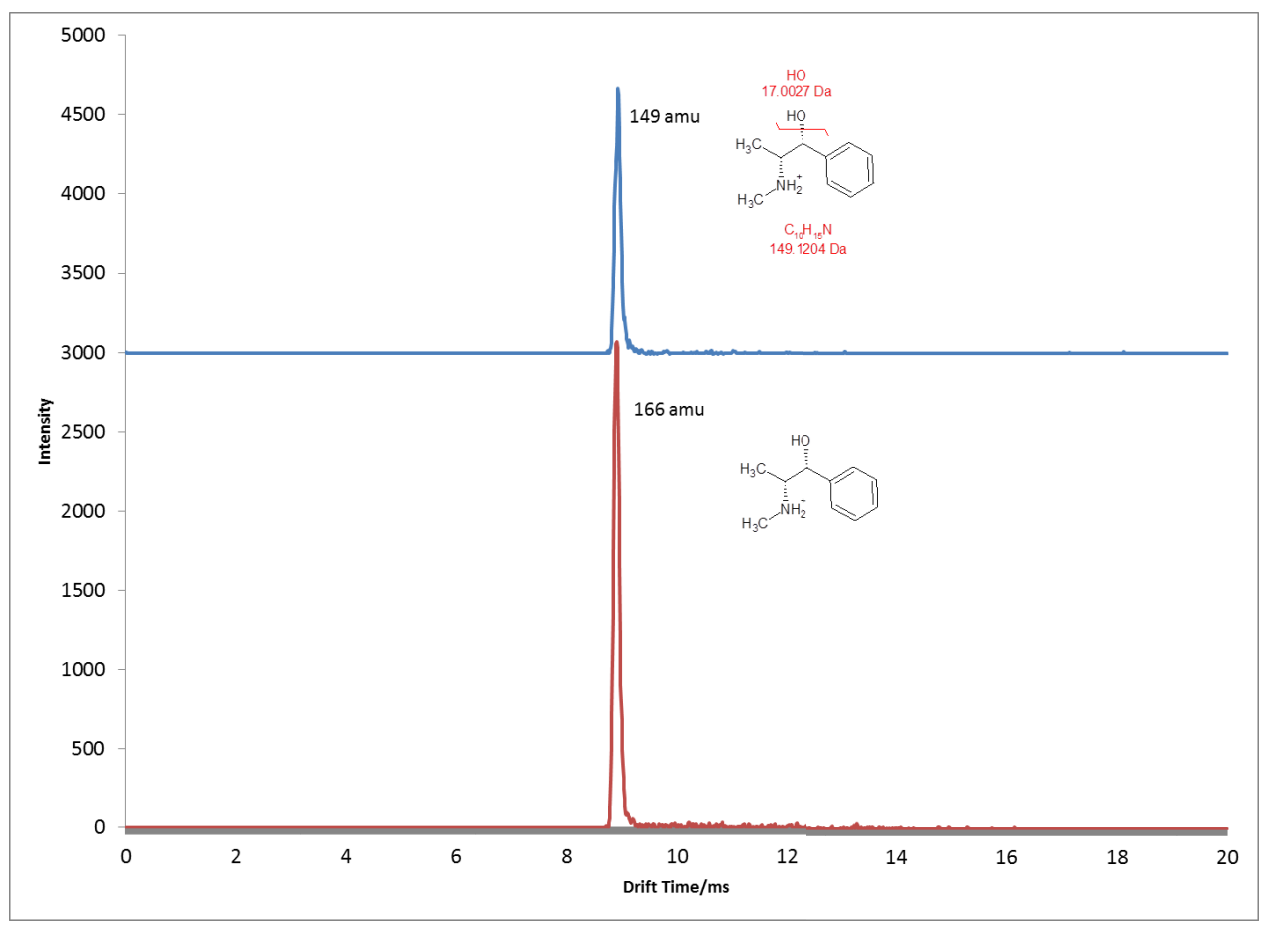

Figure 77: Single Ion Monitoring of ions from SS-Pseudoephedrine on RA4100

Similar fragmentation was observed for RS-Ephedrine, SR-Ephedrine and RRPseudoephedrine. However, when the modifier is added to the RA4100, the mass spectrum for these compounds changed. Again considering SS-Pseudoephedrine, the observed mass spectrum for this analyte when the S-2-Butanol modifier is infused into the drift tube is shown in Figure 78. Of mention here is the appearance of ion at $240 \mathrm{~m} / \mathrm{z}$ which indicates a larger ion than the expected molecular ion of SS-Pseudoephedrine. The 240 ion is an adducted species of the protonated molecular pseudoephedrine ion (166 
$\mathrm{m} / \mathrm{z}$ ) and a neutral S-2-Butanol molecule $(74 \mathrm{~m} / \mathrm{z})$. Also of interest is the lack of the 149 fragment ion previously observed in Figure 76. The larger adducted ion observed when the modifier is introduced would explain why the modifier alters the drift time of these chiral molecules.

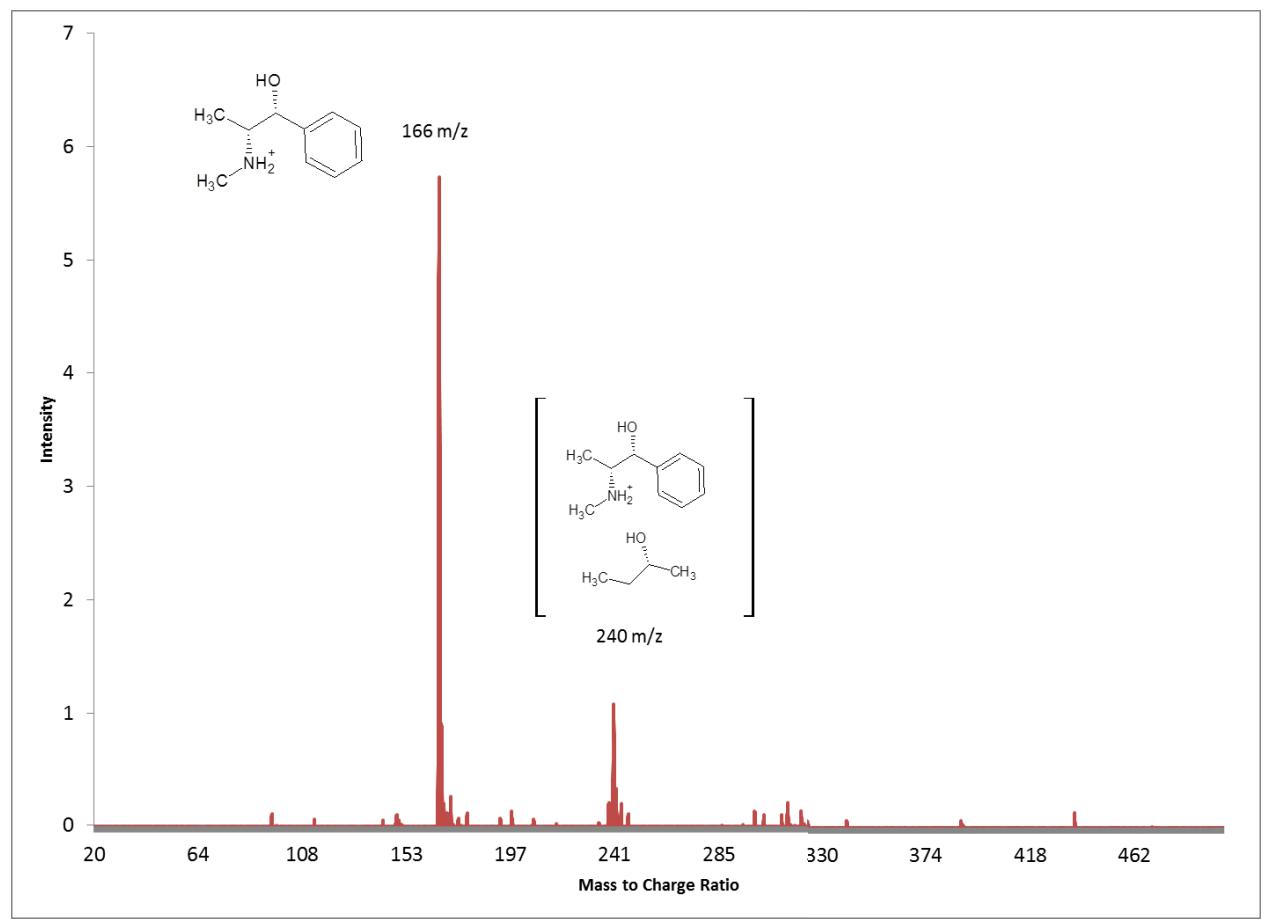

Figure 78: Mass spectrum of SS-Pseudoephedrine on RA4100 with S-2-Butanol modifier infusion

The mass spectrometer therefore provides empirical evidence that the modifier once introduced in the drift tube is interacting with the target analytes. The nature of the interaction between analyte and modifier resulting in an adducted species as revealed through mass spectrometric analysis (Figure 78) suggests that the formation of a larger ion results in retardation of the molecule that in turn changes its mobility. Other chiral modifiers were attempted such as 2,3 butanediol. However, the diol groups present on the molecule resulted in a liquid that was too viscous to be infused through a capillary 
utilizing a gas tight syringe and the boiling point of the butanediol $\left(230^{\circ} \mathrm{C}\right)$ was outside the range of the RA4100's drift tube operating temperature of $195^{\circ} \mathrm{C}$. The limited drift tube operating temperature meant that very little of the compound would vaporize into the drift tube at the standard operating temperature of $150^{\circ} \mathrm{C}$. Butanediol therefore, although attempted did not produce any experimental data. Resulting from a relatively limited supply of enantiomerically pure modifiers and high acquisition costs from the chemical manufacturer, experiments were halted. Serendipitously, alternative straight chain alcohols were then attempted starting with 2-butanol which was available in abundance. Surprisingly, separations utilizing 2-butanol gave similar results to that obtained from S-2-Butanol (Figure 79).

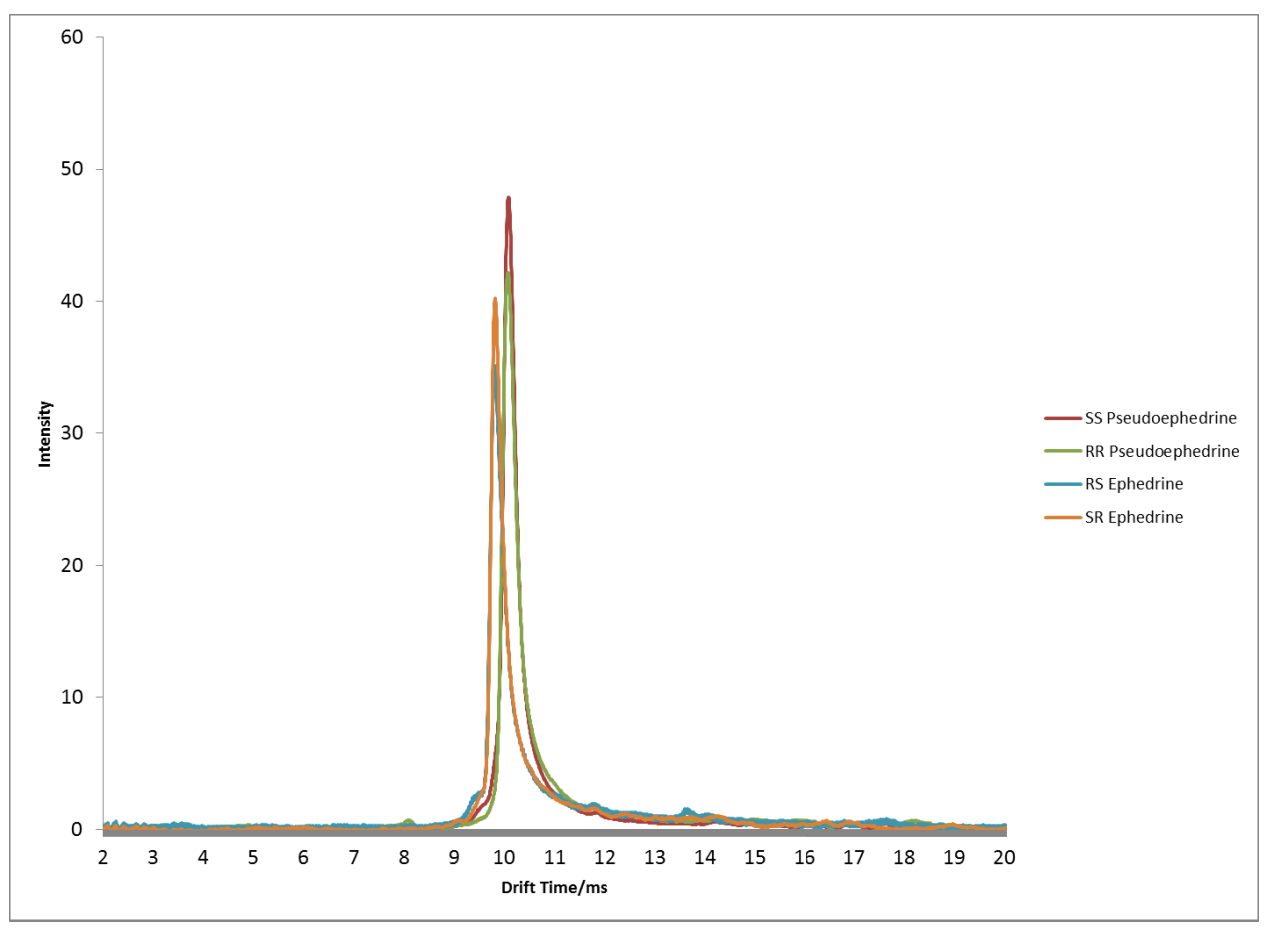

Figure 79: Separation of chiral compounds using achiral modifier 2-butanol 
With the results obtained from using an achiral modifier such as 2-Butanol, other achiral alcohols were attempted. Straight chain achiral alcohols ranging from butanol to nheptanol were used as modifiers to investigate the effects that these modifiers had on separating ephedrines and pseudoephedrines in the gas phase. The results of these experiments are shown graphically in Figure 80.

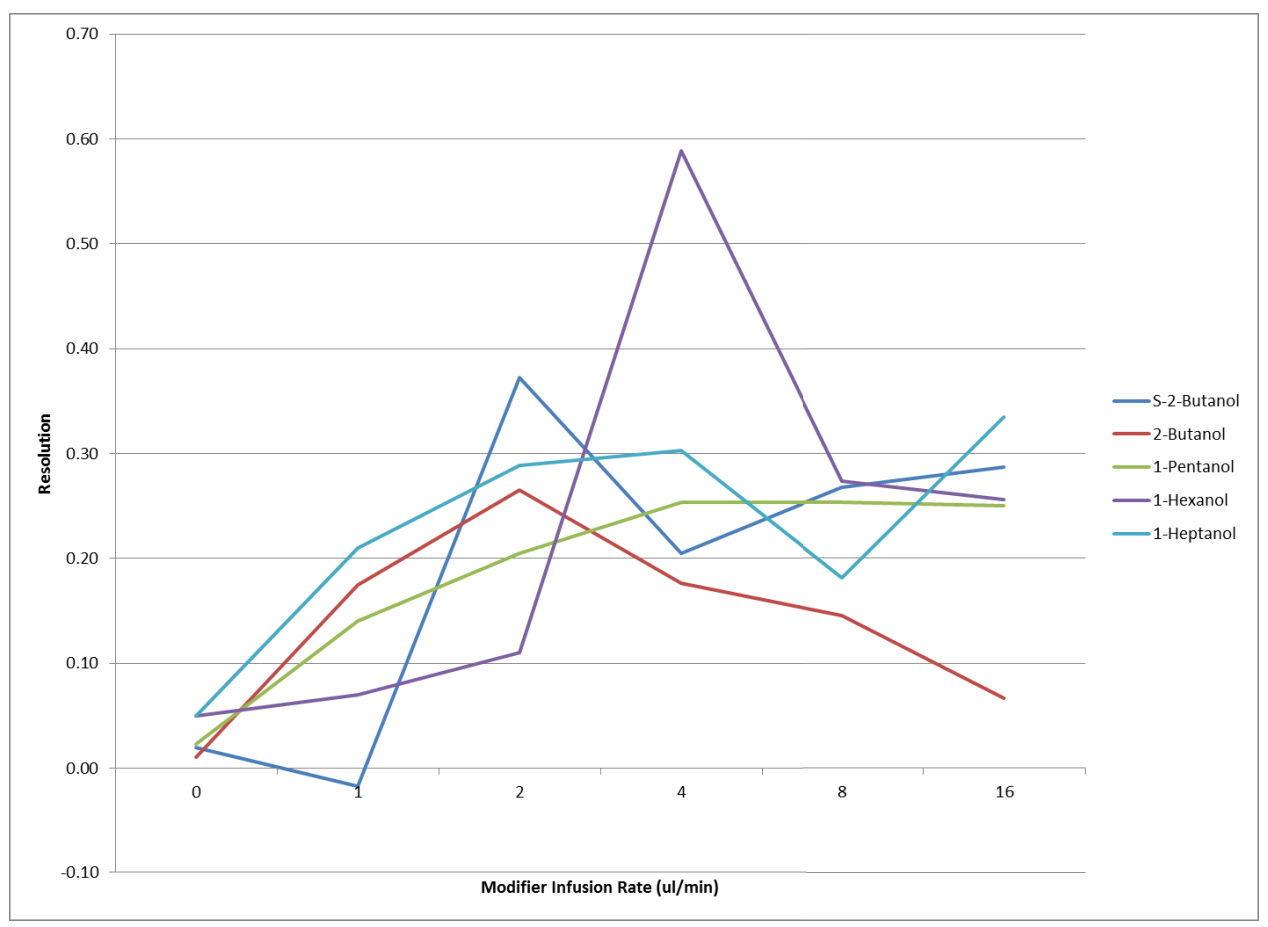

Figure 80: Resolution obtained between ephedrine and pseudoephedrine using achiral modifiers

The general trend observed is that above a particular modifier infusion rate, $4 \mu \mathrm{L}$ per minute, there is little to no enhancement in resolution capabilities, with an apparent apex in peak separating capabilities at this same $4 \mu \mathrm{L}$ per minute infusion value. Further studies commenced to investigate the exact mechanism by which the interaction between modifier and analyte altered the drift time of the target species to produce the desired separation. Previous work in this area of gas phase chiral separations utilizing ion 
mobility spectrometry $[12 ; 14 ; 15]$ hinted at a three point interaction between the chiral molecule and chiral analyte called the Pirkle rule [117] (Figure 81)

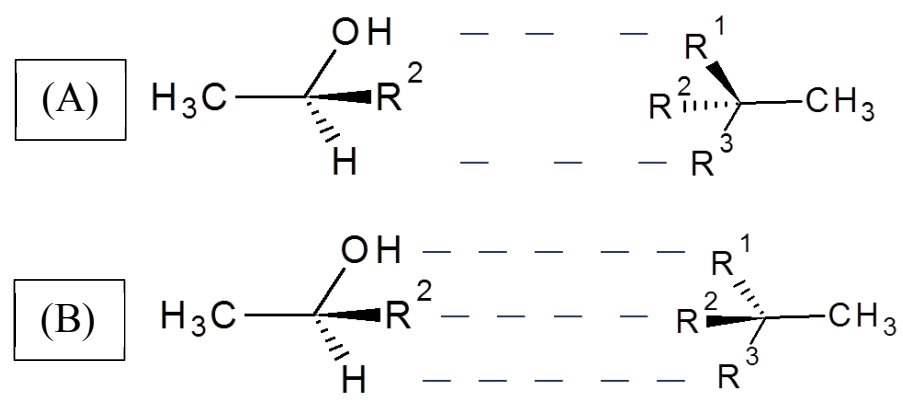

Figure 81: Adaptation of Pirkle interaction between chiral analyte and chiral modifier (a) having a weak interaction and (b) having a strong interaction.

Such separations were first reported in 1933, where it was described that three of the groups that were linked to a chiral atom were involved in the separation process [118]. Each enantiomer interacts with the modifier to a differing degree resulting in changes in the mobility of analytes that results in the desired separation. Figure 81a shows a weak interaction as not all the functional groups on the modifier are in the same plane as the functional groups on the analyte. The "weak interaction" results in a net lower interaction than that observed in Figure $81 \mathrm{~b}$ which shows all functional groups of modifier and analyte in the same plane. While the three group linkage does explain the interactions for chiral analytes and chiral modifiers, it does not however explain why the utilization of achiral modifiers would have any separating capabilities of chiral analytes as observed in Figure 80. Further studies were then conducted to investigate the mechanism and determine the rational for this seemingly inexplicable phenomenon. A full battery of tests were conducted utilizing achiral alcohol modifiers ranging from $\mathrm{C}-1$ (methanol) to $\mathrm{C}-8$ (n-octanol). Mass spectral data was collected for all analytes during modifier introduction 
to reveal the type of species being formed within the drift tube. To complement the mass spectral information obtained, physical chemical models of the detected ions were drawn. $A b$ Initio quantum chemical calculations were performed at the B3LYP/6-31g level of theory using the Gaussian 09 program suite [119]. The hybrid B3LYP [120;121] exchange-correlation functional is commonly used and well accepted in the theoretical community. 6-31g is a Pople-type basis set and is suitable for large systems, such as the analytes and modifiers displayed in this work [122]. These calculations provide energies for a certain set of geometry parameters that can be modified by making small iterations to obtain new energy values. By continuously varying the geometry parameters, an optimized geometry will be eventually achieved resulting in the lowest possible energy [123]. The structure is considered the optimized ground state structure and will yield bond lengths and bond angles with an accuracy of $0.1 \AA$ and $0.01^{\circ}$, respectively [124]. The geometry of the analytes, $(\mathrm{R}, \mathrm{S})$ and $(\mathrm{S}, \mathrm{R})$-ephedrine, and $(\mathrm{R}, \mathrm{R})$ and $(\mathrm{S}, \mathrm{S})$ pseudoephedrine, were first optimized without any modifiers to obtain structures that had intrinsic hydrogen-bonds from the oxygen atom of the hydroxyl group with one of the two hydrogen atoms of the amino group. Following the optimization, two molecules of each modifier, ranging from methanol to n-octanol, were added to the optimized analytes to allow interactions to occur for the formation of an analyte-modifier cluster. Optimized geometries calculated in vacuo of this analyte-modifier cluster indicated the optimized location of the modifiers with the interactions of the hydroxyl and amino groups of the analytes forming hydrogen-bonds. It should be noted that the number of modifier molecules was investigated from one to six, and two was found to be the ideal number of modifiers for the computational analysis due to the two functional groups of hydroxyl 
and amino present in $(\mathrm{R}, \mathrm{S})$ and $(\mathrm{S}, \mathrm{R})$-ephedrine, and $(\mathrm{R}, \mathrm{R})$ and $(\mathrm{S}, \mathrm{S})$-pseudoephedrine. The achiral modifiers were added to the drift gas of the IMS through the modifier syringe pump that was connected through a capillary tube into a heated chamber. Once introduced in the drift tube, these combined gases flow counter to the direction in which the ionized analytes migrate down the drift tube (Figure 82). The modified drift gas then interacts with the analyte to effect the desired change in mobility which results in separation.

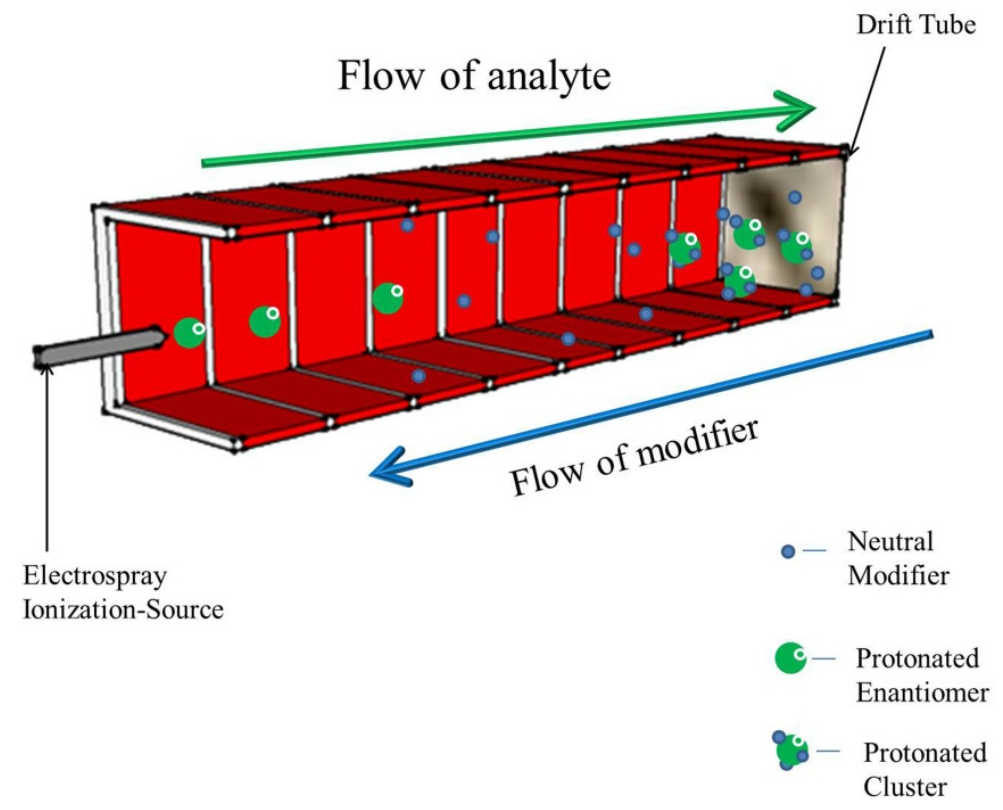

Figure 82: Representation of analyte-cluster formation in the drift tube region of IMS with modifier (Not drawn to scale, IMS gate not shown for clarity)

Results revealed that the interaction between analyte and modifier, though stereospecific, occurs without the requirement of the modifier having chiral characteristics. Achiral modifiers interacted with chiral analytes to change their mobility on the basis of hydrogen-bonding interactions of the modifier to different positions on the chiral analyte. Mass spectrometric analysis of the modified analyte in the gas phase revealed that ion 
clusters were formed as a result of numerous neutral achiral modifiers adducting to the chiral analyte (Figure 83).

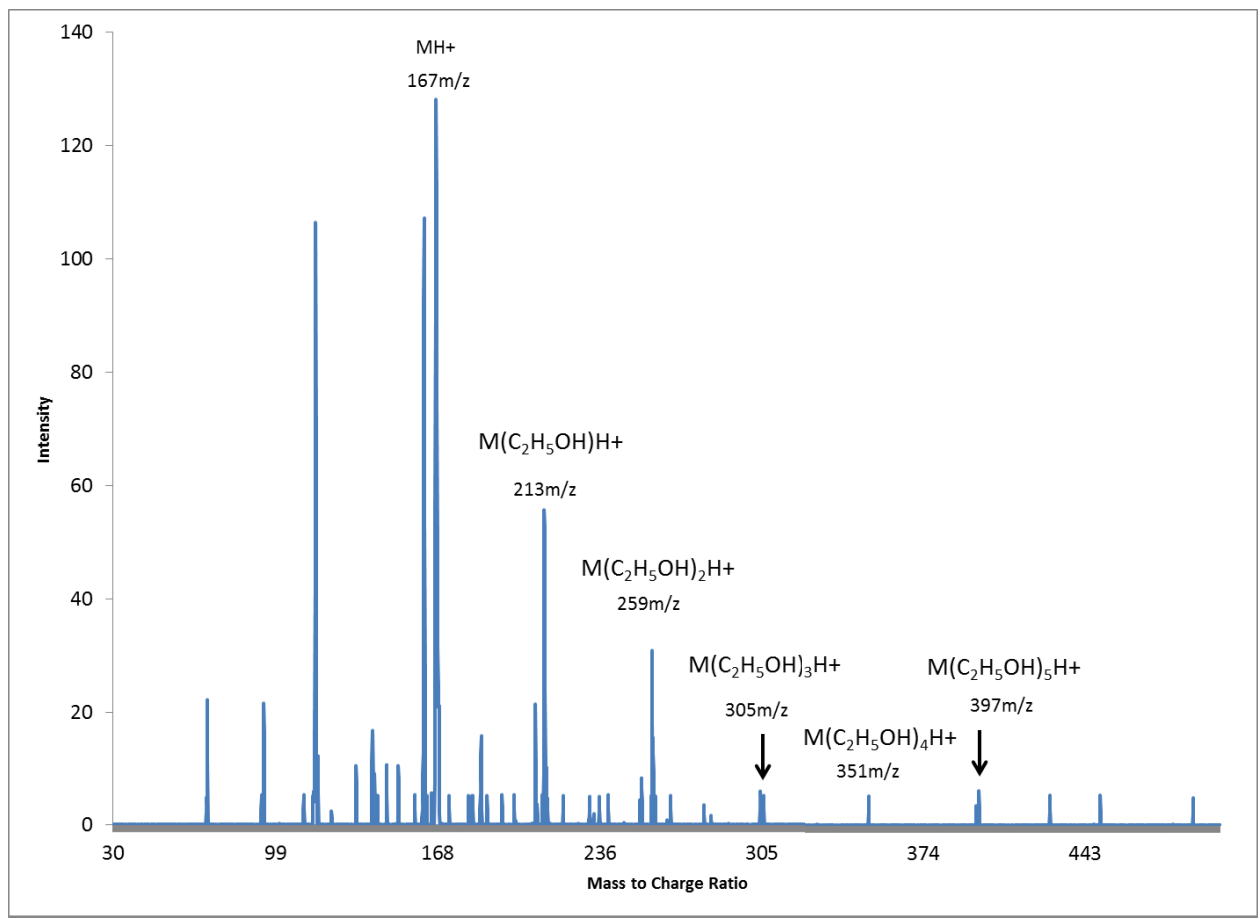

Figure 83: Mass spectrum of analyte (RS-ephedrine) with modifier clusters (ethanol)

The observation of multiple achiral modifier molecules on a single chiral analyte differs from previously reported mechanisms that have suggested that these interactions occur only between chiral analytes and chiral modifiers [125] and in addition, that these interactions occur depending on a three point interaction with one analyte to one modifier (Figure 81) [118]. Similar sized clusters were formed for each set of enantiomers; however these clusters still migrated at different drift times under the influence of the ion mobility electric field indicating that the clusters most likely had a difference in cross sectional area $(\Omega)$ which resulted in the clusters having different drift times. On the basis of the presented findings it is being proposed that the clusters formed when the achiral 
modifier was introduced in the drift tube, emphasized or exaggerated the conformational differences of each enantiomer. The conformational differences resulted in a change in the effective cross sectional area for each clustered enantiomer causing each cluster to migrate at a different rate (mobility) down the electric field. Earlier works with ion mobility involving isomeric species revealed that compounds with cross sectional differences as small as $2.5 \%$ can be successfully separated under the influence of a mild electric field [126]. These adducted modifier molecules retarded the chiral ions as they migrated down the drift tube resulting in a net increase in drift time. The observed ion clusters have a mass defined by the formula $\left(\mathrm{MH}^{+}\right) \mathrm{N}_{x}$ (Figure 84).

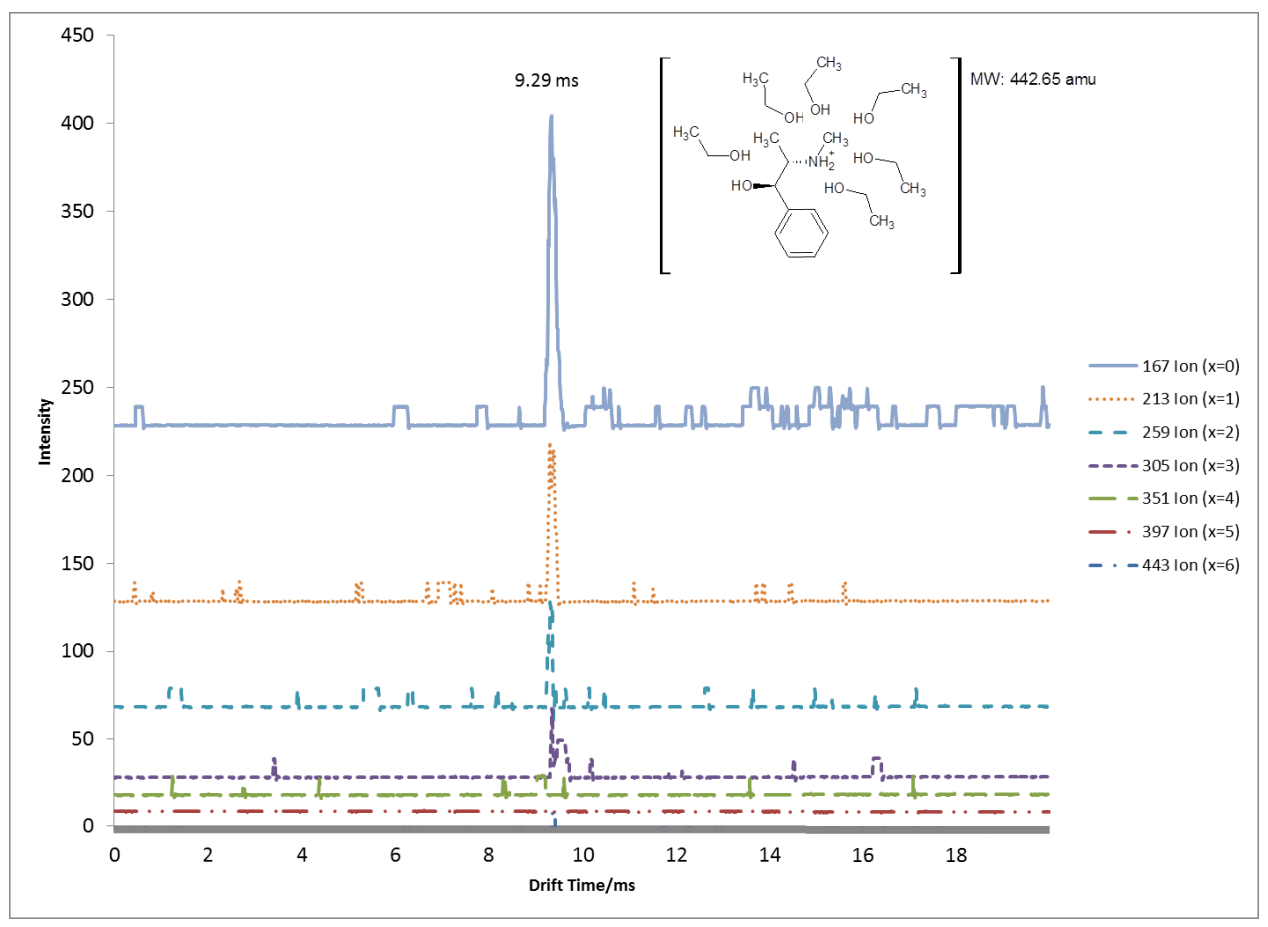

Figure 84: Single Ion monitoring of ions from analyte modifier interaction

Where $\mathrm{MH}+$ represents the mass of the protonated analyte, $\mathrm{N}$ represents the mass of the neutral adducted modifier molecule, and $\mathrm{x}$ being a positive integer that is proportional to the amount of modifier in the drift gas. Our optimized experimental parameters used a 
modifier concentration of $\sim 0.1 \% \mathrm{v} / \mathrm{v}$ modifier to drift gas (i.e., $4 \mu \mathrm{L}$ modifier in $3800 \mathrm{~mL}$ of drift gas). For a given ion cluster, $\left(\mathrm{MH}^{+}\right) \mathrm{Nx}$, a single peak with a consistent drift time was observed by the IMS faraday detector of the IMS (Figure 84), however for the same analyte peak, numerous ions of different integers of $x$ were detected by the mass spectrometer. The numerous ions detected by the mass spectrometer indicate that the ion cluster migrated intact down the IMS drift tube and then fragmented once inside the high vacuum mass spectrometer. The cluster formed is both larger in mass and cross sectional area $(\Omega)$ than the un-modified analyte resulting in a change in the mobility of the chiral analyte. The change in cross sectional area differs for each chiral analyte and results in the desired separation. A list of the modifiers used and the separation that results is shown in Table 7, while Table 8 shows the physical properties of each modifier. Enders et al. highlighted the possibility of such interactions between chiral and achiral molecules and another more recent work by Howdle et al. shows similar separation capability with a racemic modifier mixture [127;128]. The experimental results from this current work support these interactions and characterize the complex ions formed. As mass spectrometry itself is a chirally-blind technique [127], the coupling of a chiral ion mobility unit provides the required dimension of discrimination to distinguish isomeric compounds within a quadrupole mass spectrometer, allowing for each chiral analyte to be detected separately. The effect of each achiral modifier on the drift time for analytes tested is displayed in (Table 7). Discrimination between (R,S)-ephedrine and (S,S)pseudoephedrine utilizing straight chain achiral alcohols as modifiers was achieved. To further explain the results obtained between these chiral analytes and achiral modifiers, the computational model previously described was constructed for the analyte-modifier 
interactions. Density Functional Theory (DFT) calculations were performed at the B3LYP/6-31g level of theory. To each optimized analyte $(\mathrm{R}, \mathrm{S})$ and $(\mathrm{S}, \mathrm{R})$-ephedrine and $(\mathrm{R}, \mathrm{R})$ and $(\mathrm{S}, \mathrm{S})$-pseudoephedrine, two modifier molecules were added to simulate the interactions that occur for an analyte-modifier cluster. These analyte-modifier clusters, after optimization formed either a "bridged" cluster with a small cross-section or an “independent" cluster with a larger cross-section $(\Omega)$.

Table 7: Effect of modifier on resolution between (R,S)-ephedrine and (S,S)-pseudoephedrine

\begin{tabular}{|c|c|c|c|c|}
\hline & $\begin{array}{c}\text { (R,S)- } \\
\text { ephedrine } \\
\text { drift time } \\
\text { (ms) }\end{array}$ & $\begin{array}{c}\text { (S,S)- } \\
\text { pseudoephedrin } \\
\text { e drift time (ms) }\end{array}$ & $\begin{array}{c}\text { Resolution } \\
\text { between } \\
\text { analytes }\end{array}$ & $\begin{array}{c}\text { \% } \\
\text { Difference } \\
\text { in Drift } \\
\text { Time }\end{array}$ \\
\hline No Modifier & 9.012 & 9.029 & $0.09 \pm 0.20 \%$ & $0.19 \%$ \\
\hline Methanol & 9.162 & 9.21 & $0.31 \pm 0.30 \%$ & $0.52 \%$ \\
\hline Ethanol & 9.301 & 9.502 & $1.27 \pm 0.30 \%$ & $2.16 \%$ \\
\hline n-Propanol & 9.465 & 9.668 & $1.19 \pm 0.10 \%$ & $2.14 \%$ \\
\hline n-Butanol & 9.854 & 10.067 & $1.05 \pm 41 \%$ & $2.16 \%$ \\
\hline n-Pentanol & 9.984 & 10.212 & $0.84 \pm 0.48 \%$ & $2.28 \%$ \\
\hline n-Hexanol & 10.531 & 10.699 & $0.67 \pm 0.44 \%$ & $1.60 \%$ \\
\hline n-Heptanol & 10.867 & 11.171 & $1.01 \pm 0.24 \%$ & $2.80 \%$ \\
\hline n-Octanol & 10.691 & 11.186 & $1.34 \pm 0.14 \%$ & $4.63 \%$ \\
\hline
\end{tabular}


Table 8: Physical properties of modifiers used for chiral separations [129]

\begin{tabular}{|c|c|c|c|c|c|}
\hline & $\begin{array}{c}\text { Density } \\
(\mathbf{g} / \mathbf{m l})\end{array}$ & $\begin{array}{c}\text { Viscosity (Pa·s) } \mathbf{a} \\
\mathbf{2 5 C}\end{array}$ & $\begin{array}{c}\text { Boiling Point } \mathbf{(} \\
\mathbf{0} \mathbf{C})\end{array}$ & $\begin{array}{c}\text { Dielectric } \\
\text { Constants }\end{array}$ & $\begin{array}{c}\text { Dipole Moments } \\
(\boldsymbol{\mu})\end{array}$ \\
\hline Methanol & 0.7914 & 0.544 & 65 & 33 & $1.70 \pm 0.02$ \\
\hline Ethanol & 0.7893 & 1.074 & 78.5 & 25.3 & $1.69 \pm 0.03$ \\
\hline n-Propanol & 0.8035 & 1.945 & 97.4 & 20.8 & $1.55 \pm 0.03$ \\
\hline n-Butanol & 0.8098 & 2.544 & 117.2 & 17.84 & $1.66 \pm 0.03$ \\
\hline n-Pentanol & 0.8144 & 3.619 & 137.3 & 15.13 & 1.7 \\
\hline n-Hexanol & 0.8136 & 4.578 & 158 & 13.03 & N/A \\
\hline n-Heptanol & 0.8219 & 5.810 & 176 & 11.75 & N/A \\
\hline n-Octanol & 0.827 & 7.288 & 194.4 & 10.3 & 1.76 \\
\hline
\end{tabular}

For the bridged motif, the hydroxyl hydrogen of the modifier forms a hydrogen-bond with the hydroxyl oxygen of the analyte, while the hydroxyl oxygen of the modifier would form a hydrogen-bond with the amino hydrogens of the analyte. The interaction forms a "bridge" between the modifier and the two functional groups of the analyte (Figure 85).
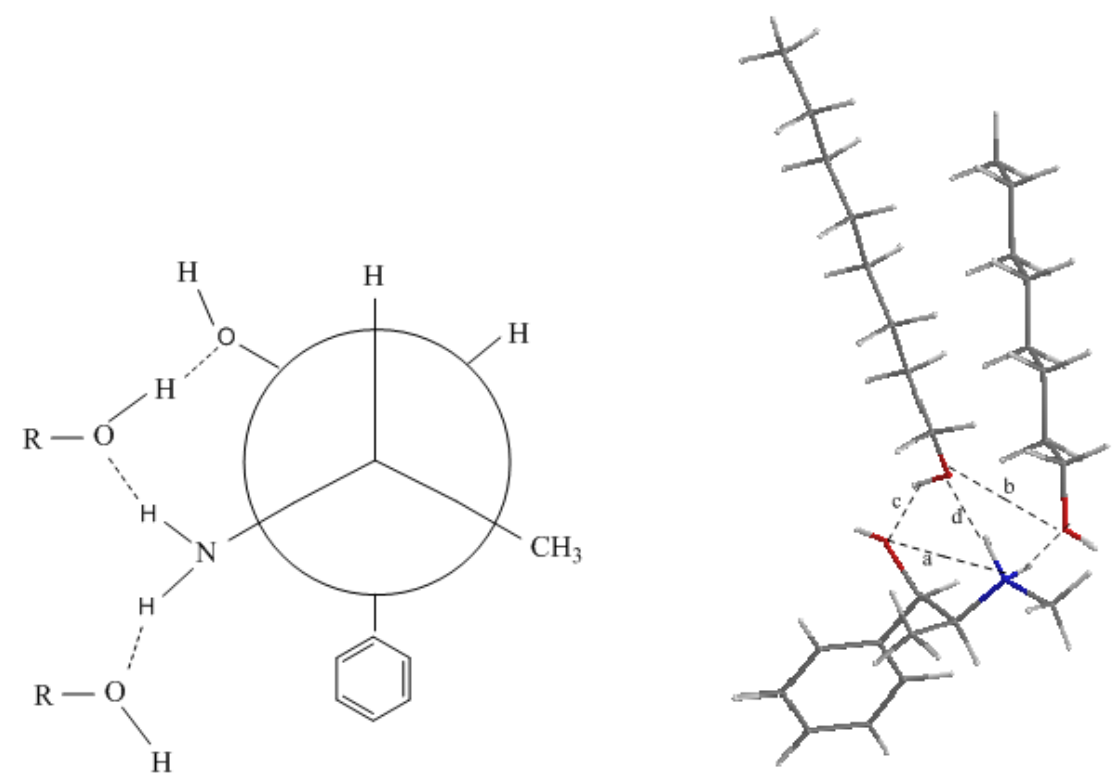

Figure 85: Newman and visual projections of (R,S)-ephedrine with two noctanol modifiers depicting the "bridged" motif or compact configuration 
The bridged motif reveals that modifiers are capable of two hydrogen-bonds with both hydroxyl and amino functional groups of the analyte, causing a condensed analytemodifier cluster that reduces its overall cross-sectional area. The "bridged" analytemodifier cluster motif was shown to occur with the analytes of $(\mathrm{R}, \mathrm{S})$ and $(\mathrm{S}, \mathrm{R})$ ephedrine. Figure 85 shows a Newman Projection of $(\mathrm{R}, \mathrm{S})$-ephedrine with two generic nalcohols. The hydroxyl and amino functional groups of (R,S)-ephedrine are gauche (or cis) to one another, and capable of having an n-alcohol fit in a highly ordered orientation with two hydrogen bonds to the one molecule of alcohol. The bridged configuration results in a smaller cross-section and consequently a lower drift time (Table 7). In contrast, the "independent" analyte-modifier cluster motif was shown with (S,S) and (R,R)-pseudoephedrine analytes (Figure 86).

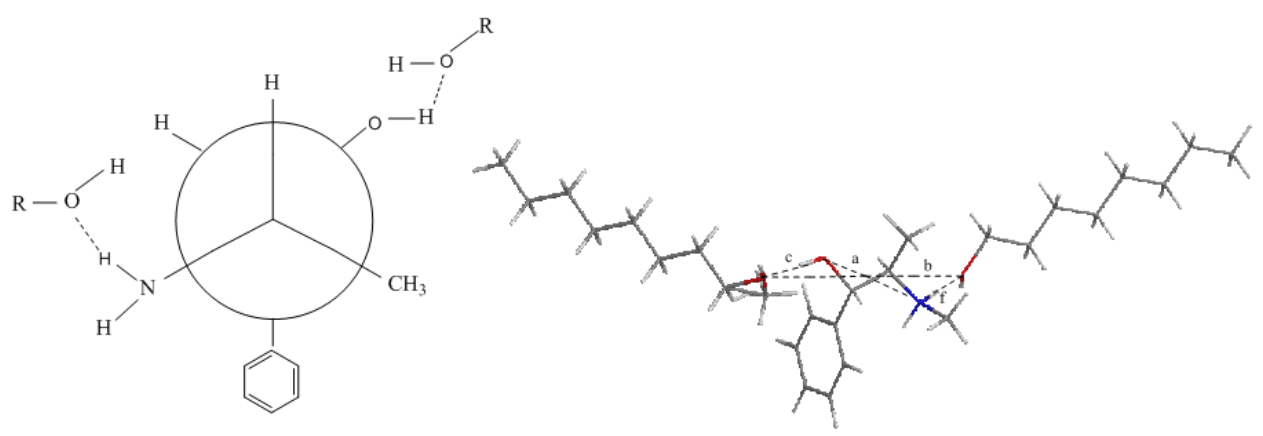

Figure 86: Newman and visual projections of (S,S)-pseudoephedrine with two noctanol modifiers depicting the "independent" motif or expanded confirmation

Figure 86 shows a Newman Projection of (S,S)-pseudoephedrine, also with two generic $\mathrm{n}$-alcohols. Both functional groups of (S,S)-pseudoephedrine are anti (or trans) to one another, resulting in an analyte-modifier cluster with a larger cross-section, and therefore higher drift time (Table 7). It should be noted that several trial initial geometries were used in placement of the modifiers around the optimized analytes, however, the 
placements depicted in this study bearing the "bridged" motif for analyte-clusters involving $(\mathrm{R}, \mathrm{S})$ and $(\mathrm{S}, \mathrm{R})$-ephedrine and the "independent" motif for the analyte-clusters involving $(\mathrm{R}, \mathrm{R})$ and $(\mathrm{S}, \mathrm{S})$-pseudoephedrine were the absolute minimum from all the initial geometry considerations. Through the utilization of several achiral modifiers, separations were obtained between (R,S)-ephedrine and (S,S)-pseudoephedrine. The best separation was obtained by using n-octanol as a modifier (Figure 87).

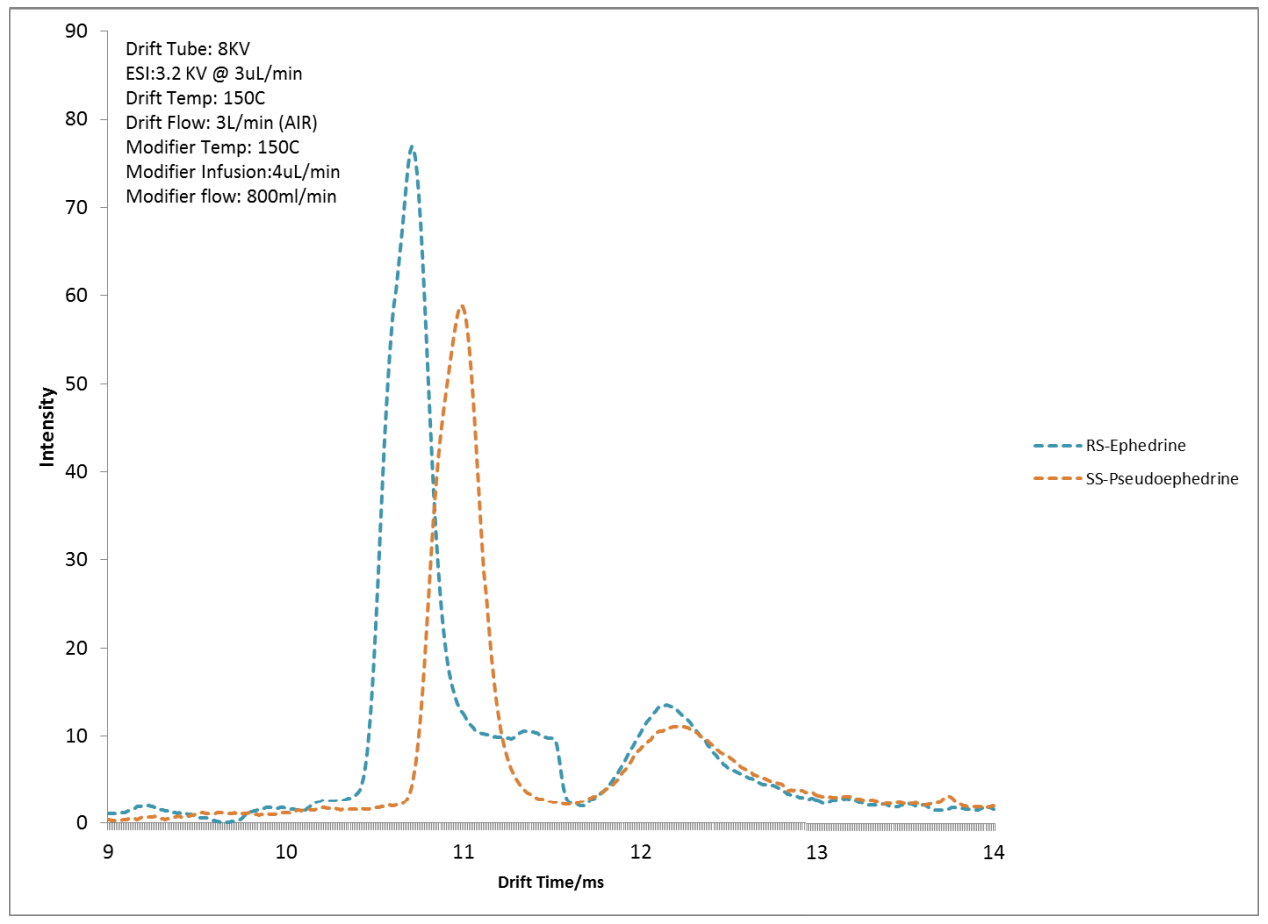

Figure 87: Separation between ephedrine and pseudoephedrine using n-octanol modifier

These analytes could not be separated without the addition of the modifiers in the gas phase as shown in Figure 88. 


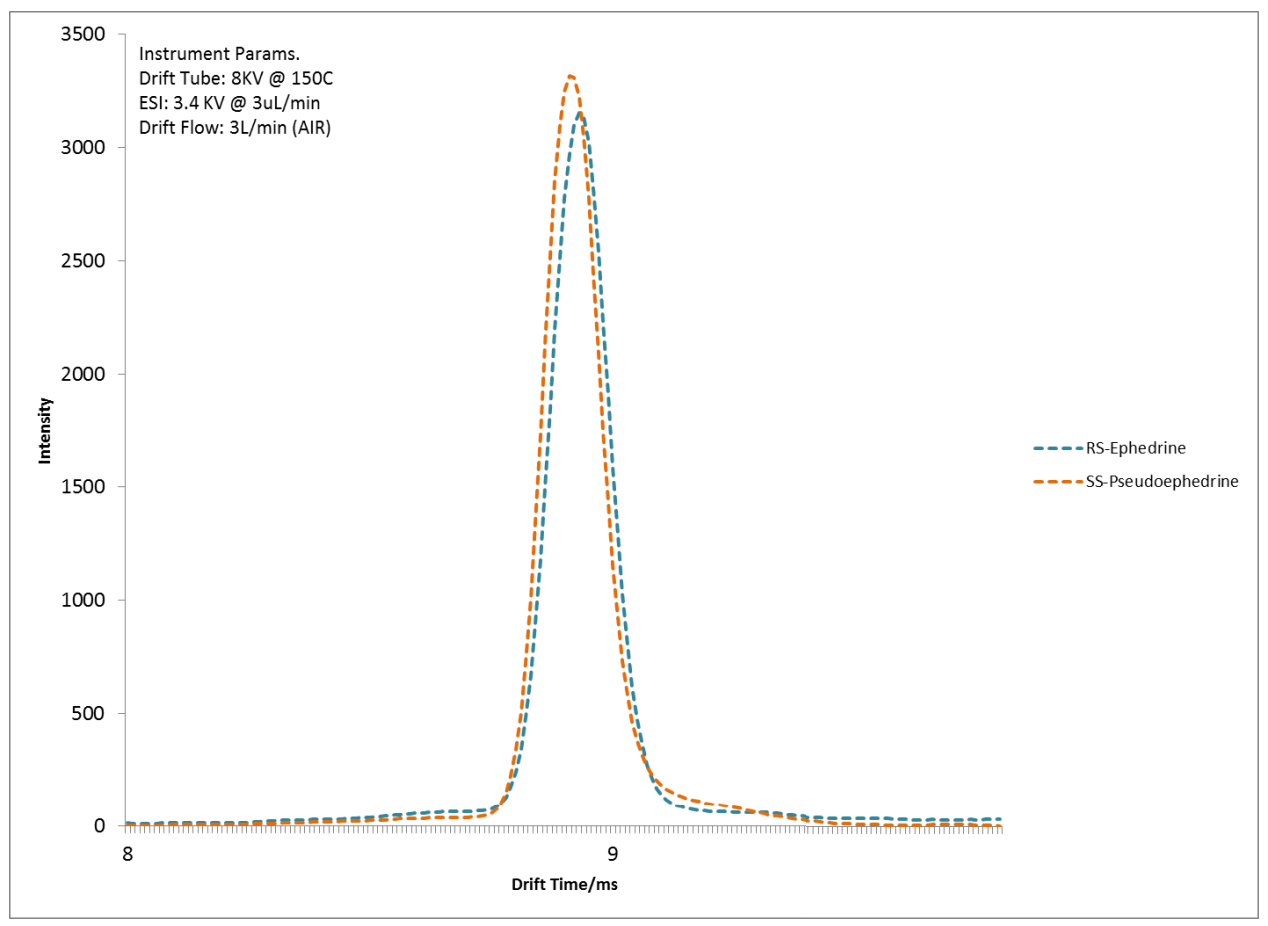

Figure 88: Analysis of ephedrine and pseudoephedrine without the use of modifier

The separation, although not with baseline resolution, has high theoretical value, particularly since the modifiers used were achiral straight chain alcohols. The ESI-IMSMS analysis has revealed that these achiral modifiers form clusters around each analyte (Figure 84) while computational calculations have revealed that the interactions between modifier and analyte were formed by hydrogen-bonding resulting in differing geometries or cross sectional areas $(\Omega)$ for each analyte cluster (Figure 85 and Figure 86 ). These differences in cross sectional area between the chiral molecule and achiral modifiers allowed for separation to occur within the IMS with very short analysis times $(<1 \mathrm{~s})$. Geometry optimizations at the B3LYP/6-31g level of theory for $(\mathrm{R}, \mathrm{S})$ and $(\mathrm{S}, \mathrm{R})$ ephedrine showed intramolecular hydrogen-bonding between the hydroxyl and amino group with internuclear distances of 3.9 and $4.6 \AA$ between analyte and modifier, 
respectively, whereas $(\mathrm{R}, \mathrm{R})$ and $(\mathrm{S}, \mathrm{S})$-pseudoephedrine showed no intramolecular hydrogen-bonds and had internuclear distances of $8.2 \AA$ between analyte and modifier. An assessment of the intrinsic hydrogen-bonds is crucial as it appears to play a role in the positioning of the modifier molecules, thereby causing a larger cross sectional area as is the case with $(\mathrm{R}, \mathrm{R})$ and $(\mathrm{S}, \mathrm{S})$-pseudoephedrine, or a smaller in the cross sectional area as seen with $(\mathrm{R}, \mathrm{S})$ and $(\mathrm{S}, \mathrm{R})$-ephedrine. These different analyte-modifier clusters had differing cross-section areas forming either a tight fit of the "bridged" analyte or wider geometry of the "independent" analyte, explaining the different drift times as was observed and reported in Table 7.

The optimized geometries of analyte-modifier clusters presented in my study represent the global energy minima [124]. The computational analysis explains the results obtained by the experimental IMS analysis, suggesting the utility of these and other modifiers for the separation of similar drugs. For my work, the greatest resolution was observed between $(\mathrm{R}, \mathrm{S})$-ephedrine and $(\mathrm{S}, \mathrm{S})$-pseudoephedrine by using $\mathrm{n}$-octanol as a modifier with a resolution of 1.34. It was also observed that as the mass of the modifier is increased (i.e. higher values of $\mathrm{N}$ ), the shift in drift time observed for each analyte is also increased (Table 7). High values of $\mathrm{N}$ did not appear to have high values of $\mathrm{x}$ for the observed ion cluster, $(\mathrm{MH}+) \mathrm{Nx}$, as detected by the mass spectrometer. The lack of detection for larger ions was largely believed to be the result of the Extrel 150's limited mass range of 500 amu. High values of both $\mathrm{N}$ and $\mathrm{x}$ would result in ions with masses above $500 \mathrm{amu}$ and hence not be observed. Despite the mass detection limitation, there exists the possibility of steric hindrance to also account for small $x$ values when $\mathrm{N}$ is large. No difference in the number of adducted molecules for a specific 
modifier was observed for any chiral analyte studied. The equal number of adducts detected for each chiral analyte indicates that each chiral analyte had the same change in overall mass when modifiers were introduced in the gas phase. It stands to reason that the differences observed in drift times were largely as a result of the differing cross sectional areas $(\Omega)$ resulting from different spatial arrangements of the modifiers around the analytes. The rational for the difference in collisional cross sectional area was confirmed by geometry optimization calculations of both $(\mathrm{R}, \mathrm{S})$ and $(\mathrm{S}, \mathrm{R})$-ephedrine and $(\mathrm{R}, \mathrm{R})$ and $(\mathrm{S}, \mathrm{S})$-pseudoephedrine with the modifiers used. High molecular weight modifiers, i.e., those with increased numbers of carbons in the n-alcohol skeleton (large $\mathrm{N}$ values), appeared to have a suppression effect on signal intensity. Intensities for analytes consistently decreased for high values of $\mathrm{N}$ when comparing un-modified analytes at $50 \mathrm{ng} / \mu \mathrm{L}$ with modified analytes at the same concentration (Figure 87 and Figure 88), signal intensities of $\sim 3500$ and $\sim 70$ were obtained respectively. Ongoing research conducted revealed that neither straight chain alkane modifiers nor modifiers with phenol functional groups had any effect on changing the mobility of these enantiomers. The interactions with the functional groups of the analytes allowed the formation of an analyte-modifier cluster that followed one of the two motifs previously described, either "bridged" or "independent" binding. The bridged motif, exhibited by clusters with $(\mathrm{R}, \mathrm{S})$ and $(\mathrm{S}, \mathrm{R})$-ephedrine, placed modifiers tightly between the two functional groups of the analyte, allowing both functional groups to have a hydrogenbond to the modifier and resulting in a small cross-section of the analyte-modifier cluster. The independent motif, exhibited by the clusters with $(\mathrm{R}, \mathrm{R})$ and $(\mathrm{S}, \mathrm{S})$-pseudoephedrine, resulted from both hydrogen-bonding functional groups of the analytes being out of plane 
from one another, causing the modifiers to be independently hydrogen-bonded to the functional groups, leading to a larger cross-section of the cluster. As the experimental results indicated, the larger cross-sectioned clusters would have a higher drift time and therefore, show separation from the smaller cross-sectioned clusters. Techniques to improve the delivery of other high molecular weight achiral modifiers will also be investigated as higher molecular weight modifiers though more effective in separating analytes, tended to be more viscous and did not disperse readily into the drift region.

\subsubsection{Conclusions:}

The separation of chiral molecules in the gas phase of an IMS using achiral modifiers is reported for the first time. A proposed mechanism by which these ion-neutral interactions take place within the IMS was probed with mass spectral analysis and the ion clusters $\left[\left(\mathrm{MH}^{+}\right) \mathrm{N}_{x}\right]$ have been identified. Where $\mathrm{MH}^{+}$represents the mass of the protonated analyte, $\mathrm{N}$ represents the mass of the neutral adducted modifier molecule, and $x$ is a positive integer that is proportional to the amount of modifier in the drift gas. Separations were obtained for $(\mathrm{R}, \mathrm{S})$-ephedrine and $(\mathrm{S}, \mathrm{S})$-pseudoephedrine with a resolution of 1.34 utilizing achiral modifiers. Theoretical calculations suggest that the (R,S) and (S,R)ephedrine form a "bridged" analyte-modifier cluster with a small cross-section, while $(\mathrm{S}, \mathrm{S})$ and $(\mathrm{R}, \mathrm{R})$-pseudoephedrine formed an "independent" analyte-modifier cluster with a larger cross-section. These analyte-modifier interactions may be used in a practical application to achieve the separation of the ephedrine from pseudoephedrine in amphetamine-type substances encountered during forensic analysis. My work improves the understanding of the mechanism of the cluster interactions while conducting gas phase chiral separations and now reveals possibilities for the further study of gas-phase 
chiral separations using CIMS with relatively common reagents and very short analysis times.

\subsection{Application of Chiral Separations to Samples}

The theoretical determination of the separation mechanism discussed in the previous section therefore allows for the analysis of real samples in a forensic setting. Determination of the ratio of the enantiomeric make-up of these target compounds within a sample can be used for provenance approximation of seized amphetamine type substances (ATS). Methamphetamine and other amphetamine type substances (ATS) are commonly produced in clandestine laboratories by any of a dozen chemical synthetic routes [130]. Most of these routes involve a reductive approach where either ephedrine or pseudoephedrine is reduced to methamphetamine. The enantiomers of ephedrine and pseudoephedrine possess the same stereochemistry as Methamphetamine, differing only by the presence of a benzyl hydroxyl group. It has been reported that depending on the starting material used, a particular enantiomer of methamphetamine will be produced as well as remnants of the starting materials or precursor compounds used for the synthesis, since these reactions are not $100 \%$ efficient. The controlled drug (S)-methamphetamine is produced predominantly from $(\mathrm{R}, \mathrm{S})$ Ephedrine and $(\mathrm{S}, \mathrm{S})$ Pseudoephedrine while using $(\mathrm{S}, \mathrm{R})$-Ephedrine and (R,R)-Pseudoephedrine will produce predominantly (R)Methamphetamine which is not controlled [11] and sold as the active ingredient in over the counter nasal inhalers. The detection of these enantiomers can therefore yield valuable forensic insight as to the potency of the product, whether controlled or not and the method of synthesis. The United Nations Office on Drug and Crime released its 2009 
World Drug report in which it reported 10 additional countries now produce amphetamine type substances where these countries previously had no history of producing amphetamine type substances (ATS) [19]. This indicates the unique problem in the production of amphetamine type substances, where they may be produced "virtually anywhere at relatively low cost [19]." Unlike cocaine, heroin and cannabis which are plant based or natural drugs; ATS production is not restricted to areas of arable land cultivation as these are synthetically manufactured from a variety of precursor compounds. The availability and use of a particular type of precursor compound has resulted in regional trends of ATS compositions, such as predominantly one type of enantiomer being seized in Asia and Japan and racemic mixtures being seized in Europe and North America [25]. This is shown in Figure 89 with results determined through laborious and difficult chiral separations by capillary electrophoresis. 


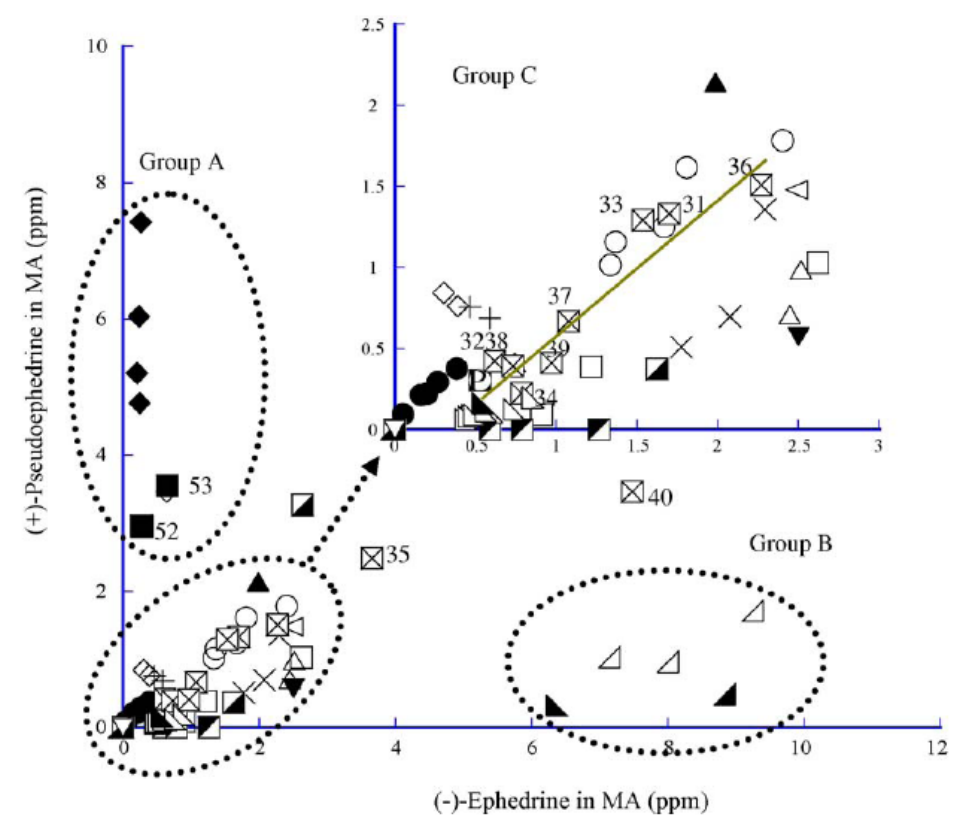

Figure 89: Ratio of chiral impurities found in ATS showing three different groups of origin A,B and $\mathrm{C}$ as determined by Capillary Electrophoresis [25].

These characteristics however go un-noticed as they may only be revealed through chiral analysis of the seized substances. This type of chiral analysis has long been an expensive and time consuming analytical technique, added to the fact that between 230 and 640 MT of illicit ATS were estimated to be manufactured globally in 2007[19], a rapid and cost effective approach is desperately needed in order to aid law enforcement to monitor sources of supply, drug trafficking routes and connections between different batches of these seized drugs. This has been previously achieved utilizing gas [24;131;132], liquid $[11 ; 117 ; 133 ; 134]$ chromatography and capillary electrophoretic [23;25;132;135-141] techniques. Peer reviewed reports on chiral separations have also been previously published from authors such as Ward et al. [142] and Fanali [143]. However, these techniques have yet to find widespread acceptance within the forensic arena and routine chiral analysis of ATS during criminal proceedings is still not a common practice. As 
previously discussed in the section titled Application of Chiral Separations to Samples (pg.141), the capabilities of the RA4100 system to conduct chiral analysis will allow for the detection of chiral impurities found in ATS by fiscally restrained law enforcement agencies through its lower operational costs.

As the mechanism of separation for these target analytes was revealed to be as a result of an interaction between the alpha hydroxyl group and the nitrogen of the beta amino group on the target chiral compounds, a compound that does not have this molecular structure could therefore be used as an internal standard. This internal standard would serve as a reference point from which the relative drift time of the chiral analytes can be determined. Cocaine was selected as this internal standard as it does not have the internal hydroxyl group on an alpha carbon in close proximity to an amino group and should therefore not be affected by a modifier during chiral IMS analysis (Figure 90).

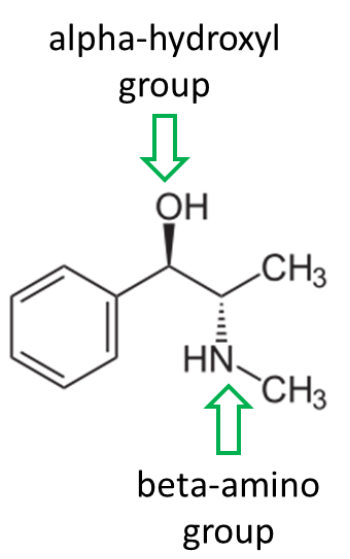

RS-Ephedrine
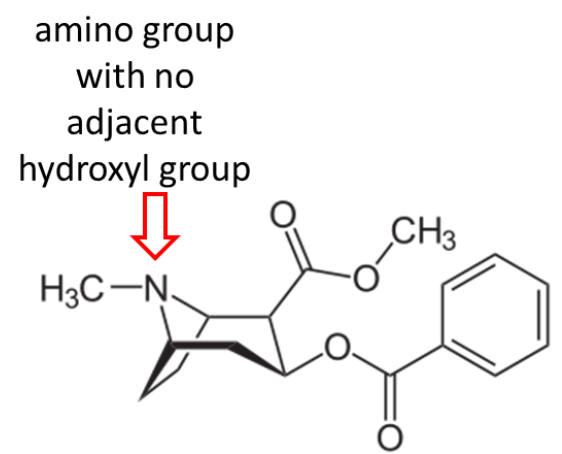

Figure 90: Differences in structure for target chiral compound ephedrine and internal standard cocaine. 
By combining the knowledge of solvent chemistry previously determined in the section Analysis of Multiple Component Mixtures Utilizing ESI-IMS-MS (pg. 98), it was possible to simultaneously analyze target analytes and cocaine in a single run (Figure 91).

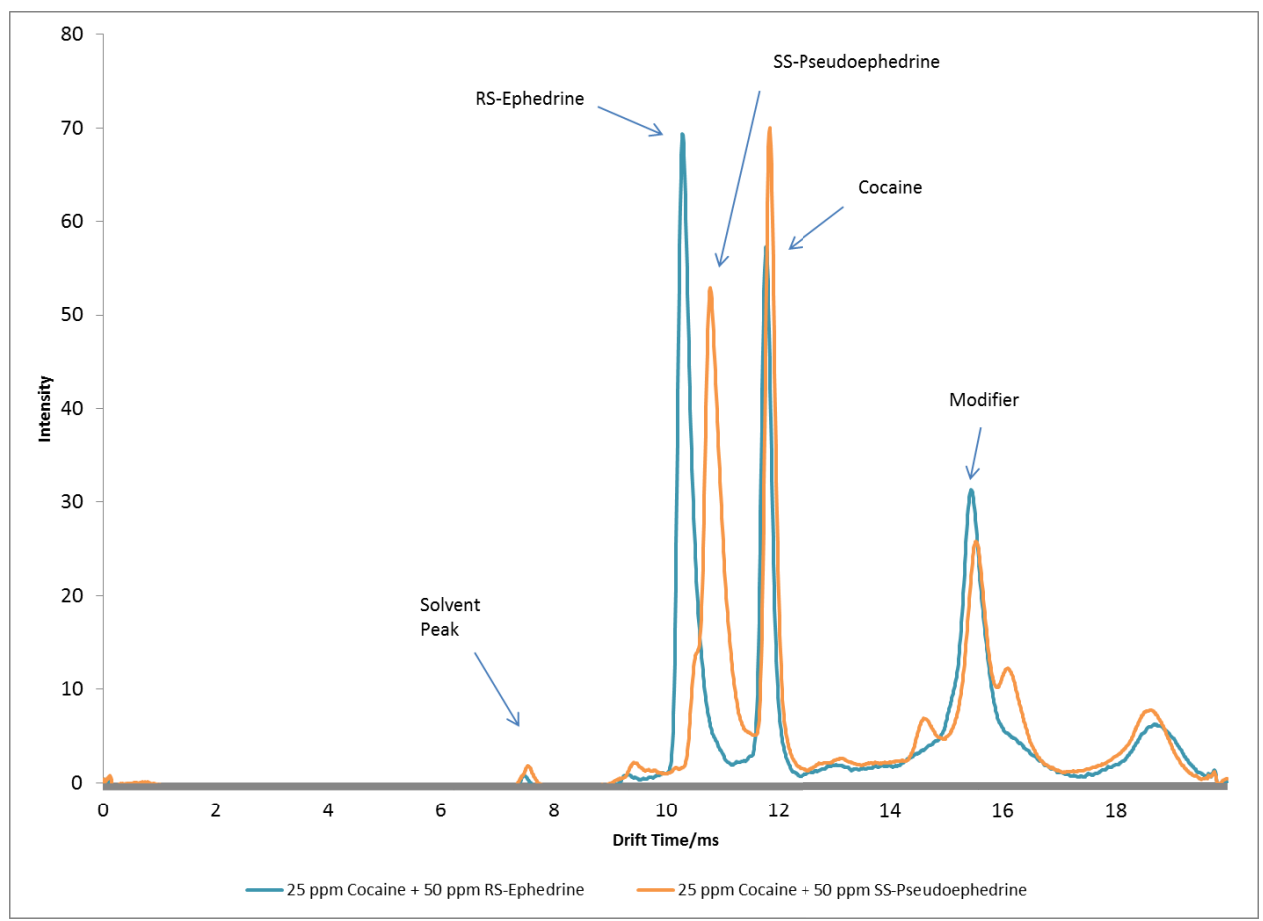

Figure 91: Analysis of chiral compounds with cocaine internal standard on RA4100

By conducting a series of these analysis utilizing chiral compounds and using cocaine as an internal standard with various achiral modifiers, a trend line was constructed of their relative drift times to cocaine (Figure 92). Figure 92 shows that while each chiral analyte was affected by the modifier being used resulting in a change in its drift time, cocaine's drift time remained constant at $11.9 \mathrm{~ms}$. This not only shows the suitability of cocaine as an internal standard but more importantly verifies the hypothesis for the chiral separation mechanism as the theory proposed accurately predicted that there should be interactions 
with compounds possessing the alpha-beta linkage shown in Figure 90, and no interactions for compounds such as cocaine that do not possess this linkage.

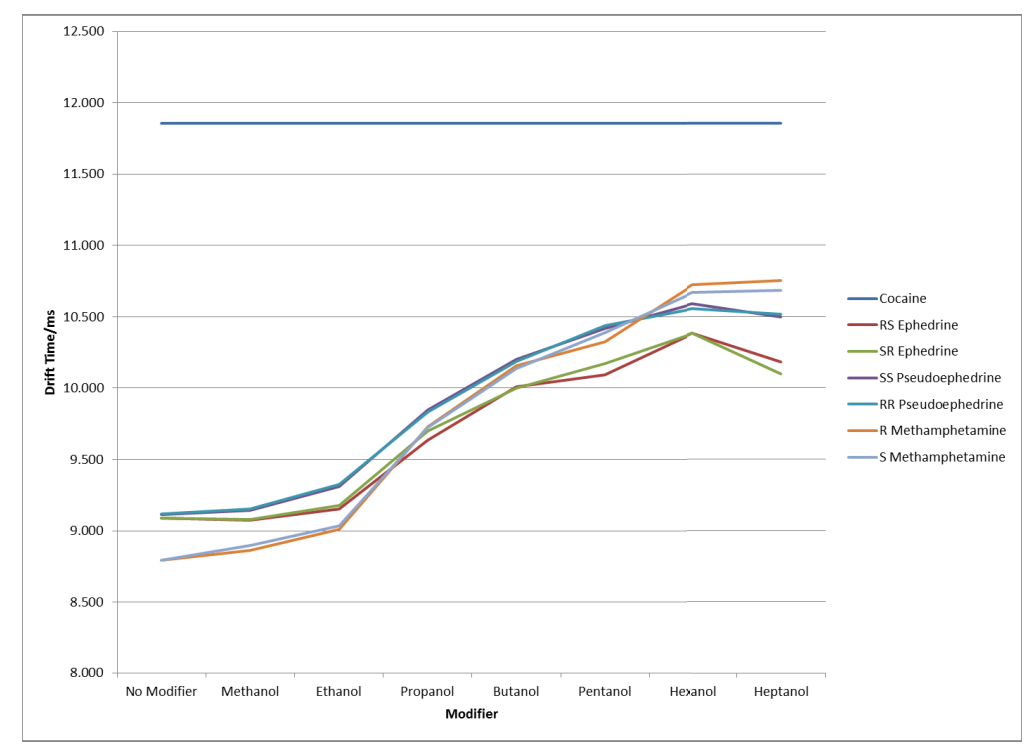

Figure 92: Drift time of analytes relative to cocaine internal standard utilizing various modifiers

The drift times of these analytes utilizing these achiral modifiers were also determined, again utilizing cocaine as the reference standard. By applying Equation 10, the reduced mobility values for each analyte was determined and plotted for all achiral modifiers used (Figure 93). 


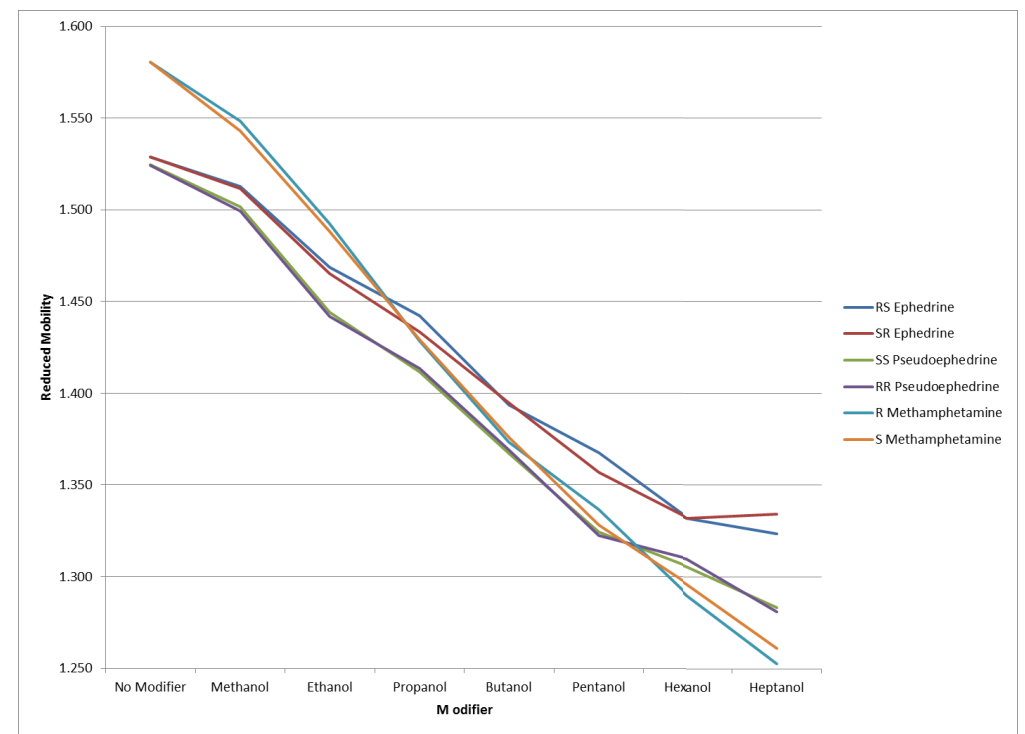

Figure 93: Reduced mobility values for chiral compounds utilizing various modifiers

Figure 93 shows that the reduced mobility values for all chiral analytes have been changed as the modifiers were altered. Though octanol did show the greatest separation for these analytes, its use was subsequently eliminated. The relatively high boiling point for octanol resulted in much of the modifier condensing in the RA4100 drift tube. The inability for the drift tube to exceed the boiling point of the modifier resulted in accumulation of the modifier within the drift tube over time, causing contamination of subsequent samples and requiring excessive down time for cleaning of the instrument that greatly reduced productivity. The demonstration of these separations is the sine qua non for analysis of real samples. As Iwata et al. demonstrated in Figure 89, the analysis and subsequent determination of ratios of these chiral compounds is key to the provenance of drugs within the forensic laboratory. Figure 94 shows the result of conducting both chiral analysis (using a modifier) and non-chiral analysis (no modifier) for a mixture of RSEphedrine, SS-Pseudoephedrine and Cocaine. 


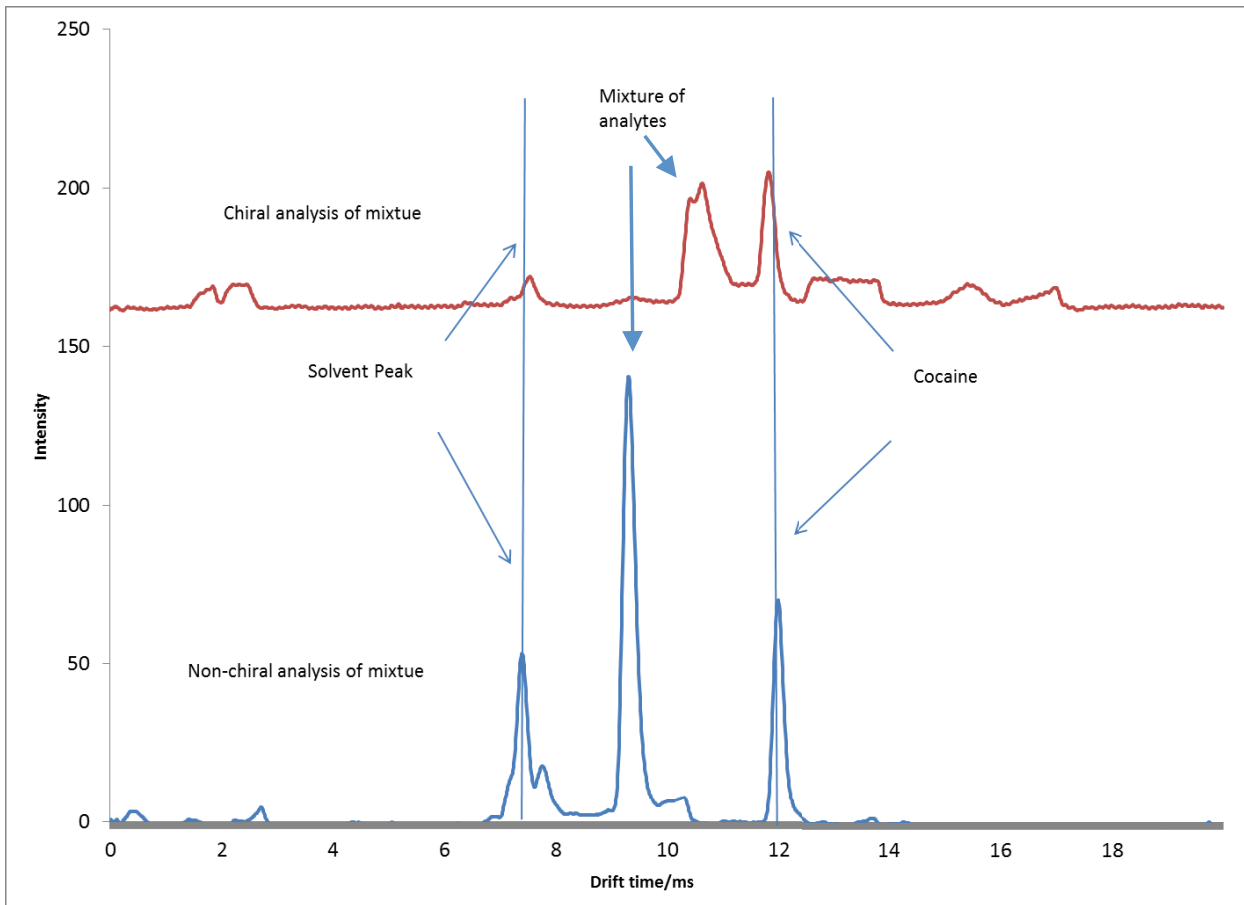

Figure 94: Analysis of a mixture of analytes using both chiral and non-chiral analysis on RA4100

For the non-chiral analysis there is complete overlap of the target analytes ephedrine and pseudoephedrine, as expected. While for the chiral analysis there is separation also as expected (Figure 94). The ability to quantify these analytes independent of one another while they are in the same mixture with this level of separation is essential. By utilizing Peak simple software, Gaussian areas can be approximated for this level of separation. Experiments of several mixtures of these chiral analytes were analyzed under chiral conditions and the concentration of each was able to be determined to provide a ratio of the analytes to one another (Figure 95). Though no actual seized drugs samples could be obtained to validate the true ability of this technique, the theoretical approach has been proven and is now available for practical deployment. 


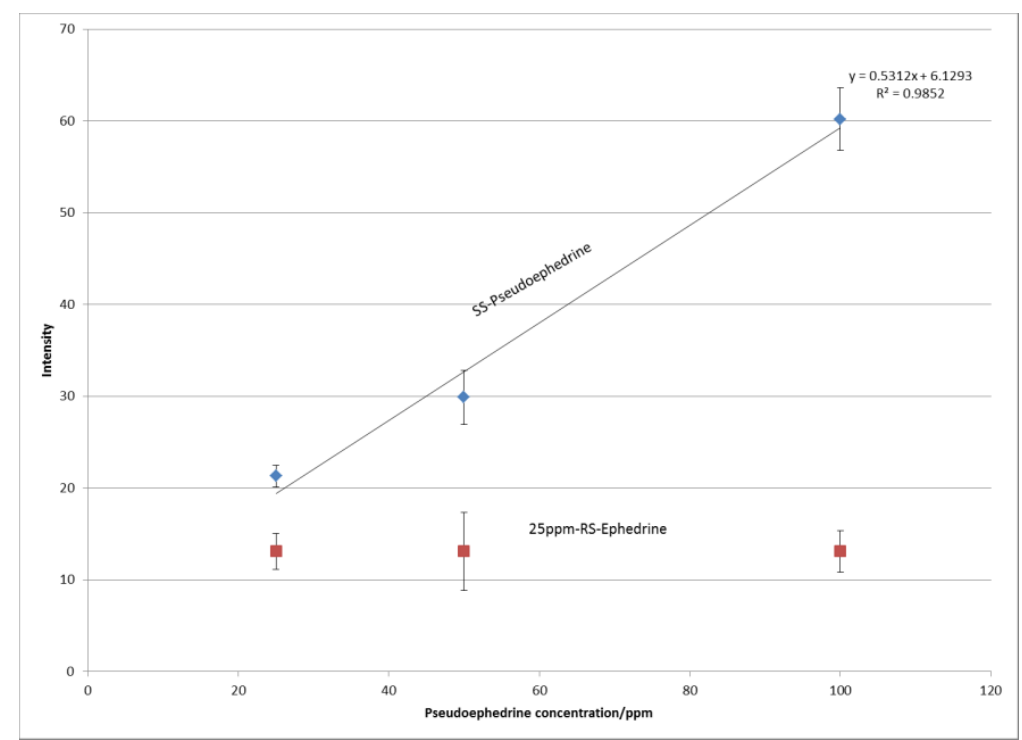

Figure 95: Determination of ephedrine and pseudoephedrine ratio from a single mixture utilizing chiral analysis on RA4100

\subsubsection{Conclusions:}

These findings therefore prove that the chiral separation is not only theoretical but applicable to mixtures through the combination of knowledge of the solvent system used for analysis which allows for these analytes to be detected simultaneously in one mixture. The use of achiral modifiers is also possible as the separation mechanism does not rely on the chiral nature of the modifier being used in order to affect the separation of analytes. Lastly, knowledge of the separation mechanism which allowed for the successful selection of an internal standard (cocaine) to validate the changes in drift time being observed for chiral analysis also validates the mechanism of the chiral separation occurring. The end result now being a functional method that may be applied to real casework in the determination of chiral impurities RS-ephedrine and SS-pseudoephedrine from amphetamine type substances that will allow forensic laboratories to conduct drug provenance on routine seized ATS samples in a cost effective manner. 


\subsection{Development of a Suitable IMS methods for the detection of Thermally Labile Explosive Compounds.}

Thermally labile compounds, are those which are easily destroyed by heat. Explosive samples typically possess this characteristic and have been difficult to analyze through conventional means such as gas chromatography as a result of the high temperatures utilized for separation and detection of analytes using this technique. As indicated by Makinen et al., GC is only able to analyze thermastable compounds that can withstand temperatures up to $300^{\circ} \mathrm{C}$ [36]. Ion mobility spectrometry however, operates at much lower (milder) temperatures that often do not result in degradation of these thermally labile analytes. The ability of IMS to perform these analyses has resulted in a plethora of ion mobility detectors deployed in a variety of field and laboratory based explosive detection operations [37;144-153]. The utilization of Electrospray Ionization based Ion Mobility builds on this trend by offering a softer ionization alternative that still maintains the integrity of the target thermally labile analytes, but also provides the advantage of enhancing the detection of these analytes through the manipulation of solvent chemistries. Several explosive molecules were examined through the course of this work. These included compounds commonly found in low explosives such as smokeless powders as these compounds are commonly used in acts of domestic terrorism or nonmilitary based incidents. The compounds found in high explosive devices were analyzed to simulate the samples that would need to be analyzed following detonation of munitions used in military based incidents. A list of these compounds and there classification has been included (Table 9). 
Table 9: Explosive Compounds and Classification

\begin{tabular}{|c|c|c|c|}
\hline Compound & Classification & $\begin{array}{l}\text { Molecular } \\
\text { Mass }\end{array}$ & Molecular Formula \\
\hline $\begin{array}{c}\text { 2,4,6-Trinitrotoluene } \\
\text { (TNT) }\end{array}$ & Nitro-Aromatic & 227.13 & $\mathrm{H}_{2} \quad \mathrm{O}$ \\
\hline $\begin{array}{l}\text { 1,3,5-Trinitro-1,3,5- } \\
\text { triazacyclohexane (RDX) }\end{array}$ & Nitro-Amine & 222.12 & \\
\hline $\begin{array}{l}\text { Octahydro-1,3,5,7- } \\
\text { tetranitro-1,3,5,7- } \\
\text { tetrazocine (HMX) }\end{array}$ & Nitro-Amine & 296.15 & \\
\hline Nitroglycerin (NG) & Nitrate-ester & 227.08 & {$\left[0^{1 /}\right.$} \\
\hline $\begin{array}{c}\text { Triacetonetriperoxide } \\
\text { (TATP) }\end{array}$ & Peroxide & 222.23 & 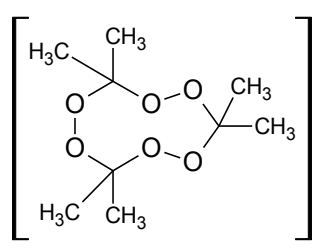 \\
\hline
\end{tabular}




\subsubsection{Classification of Explosives}

Explosives may be grouped into two main classes, either High explosives or Low explosives. High explosives are those compounds that have a burn rate above that of the speed of sound (km's/second), this high burn rate is referred to as detonation. These compounds therefore create a shockwave once ignited in addition to a large release of heat and pressure. This results in high explosives being very physically destructive, having high brisance, without the need for confinement. Low explosives on the other hand are those compounds that have a burn rate below that of the speed of sound (cm's/second), this lower burn rate is called deflagration. The slower burn rate of low explosives requires that these materials be confined during ignition in order for there to be significant destruction through physical release of heat and pressure. As a result, low explosives are found in applications such as ammunition manufacture, such as bullets, in the form of smokeless powders. These are encased and confined in casings or shells, when ignited the buildup of heat and pressure will propel the bullet out of the firearm. Malicious use of low explosives has resulted from their inclusion in improvised explosive devices (IED's). A snapshot of the global incidents involving explosives reveals a high percentage of these incidents occurring domestically within the United States (Figure 96). 


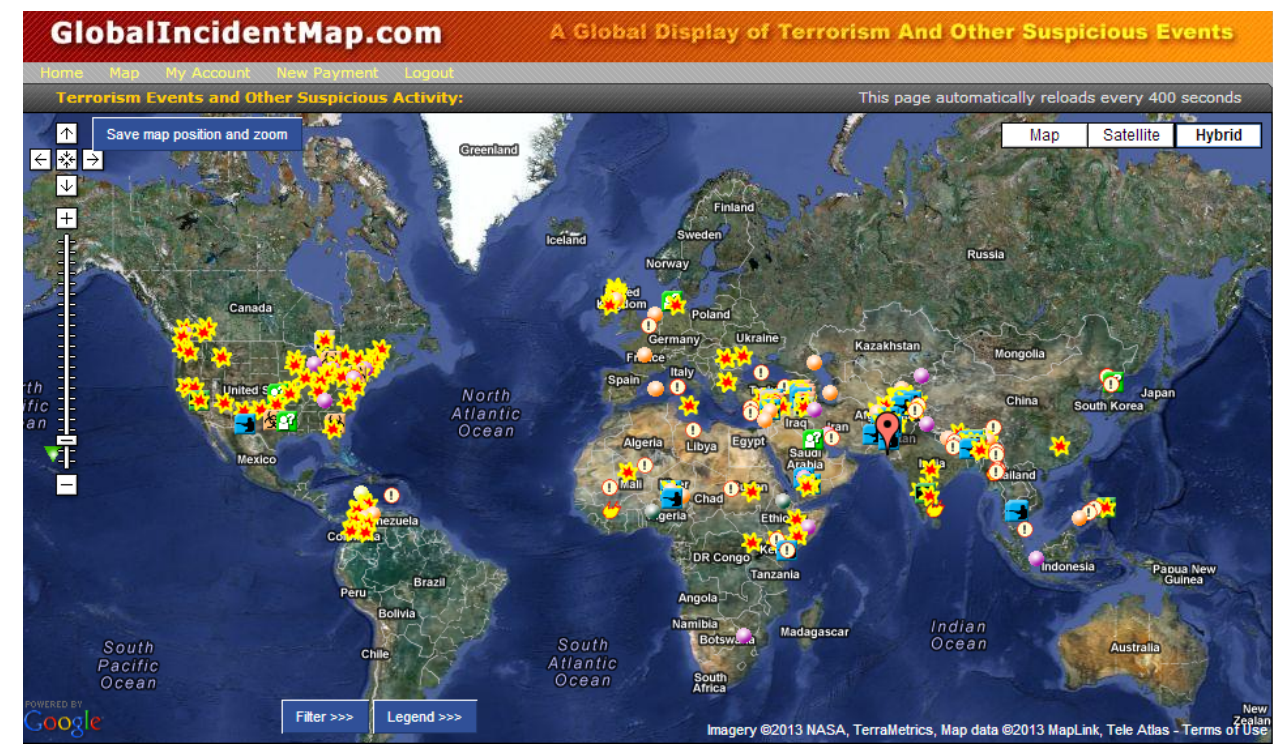

Figure 96: World Incident Map of Explosive Use (www.globalincidentmap.com)

These incidents range from false alarms where police are called and no explosive devices are found, to bomb squads finding and disabling an actual device such as a pipe bomb, generally filled with low explosive smokeless powders. At the time of this writing, the Boston Marathon bombing incident was reported utilizing two improvised explosive devices (IED's) comprised of kitchen pressure cookers loaded with explosive materials, ball bearings and nails as shrapnel, the use of which caused the deaths of 3 persons and injured several hundred [154]. The type of explosive used in this incident is still yet to be determined, but is suspected to be an easily accessible low explosive such as smokeless powders. The high occurrence of these incidents domestically has warranted the continued high alert rate of law enforcement as well as the increased need for law enforcement to be able to detect these explosive compounds in the event that they are indeed present. The non-controlled nature of low explosive materials coupled with their wide availability continues to make low explosive compounds a priority for any domestic 
effort to control or enforce explosive regulations. The compounds listed in Table 9, represent the compounds most commonly found in explosive devices, however, there exists a variety of other compounds commonly used in low explosive devices which are themselves not explosive, yet, are reliable indicators for the presence of the actual explosive compound. These compounds have been proven through many previous studies to be readily detected by Ion Mobility $[3 ; 27 ; 29 ; 30 ; 37 ; 144-146 ; 148]$. These additives which are non-energetic in nature (non-explosive) are added to these low explosives during manufacturing as stabilizers, plasticizers, flash suppressants, deterrents and opacifiers to increase stability [147]. A table showing a list of these non-energetic compounds targeted for analysis and detection of explosives is given in Table 10. 
Table 10: Non-energetic materials found in Low Explosive smokeless powders

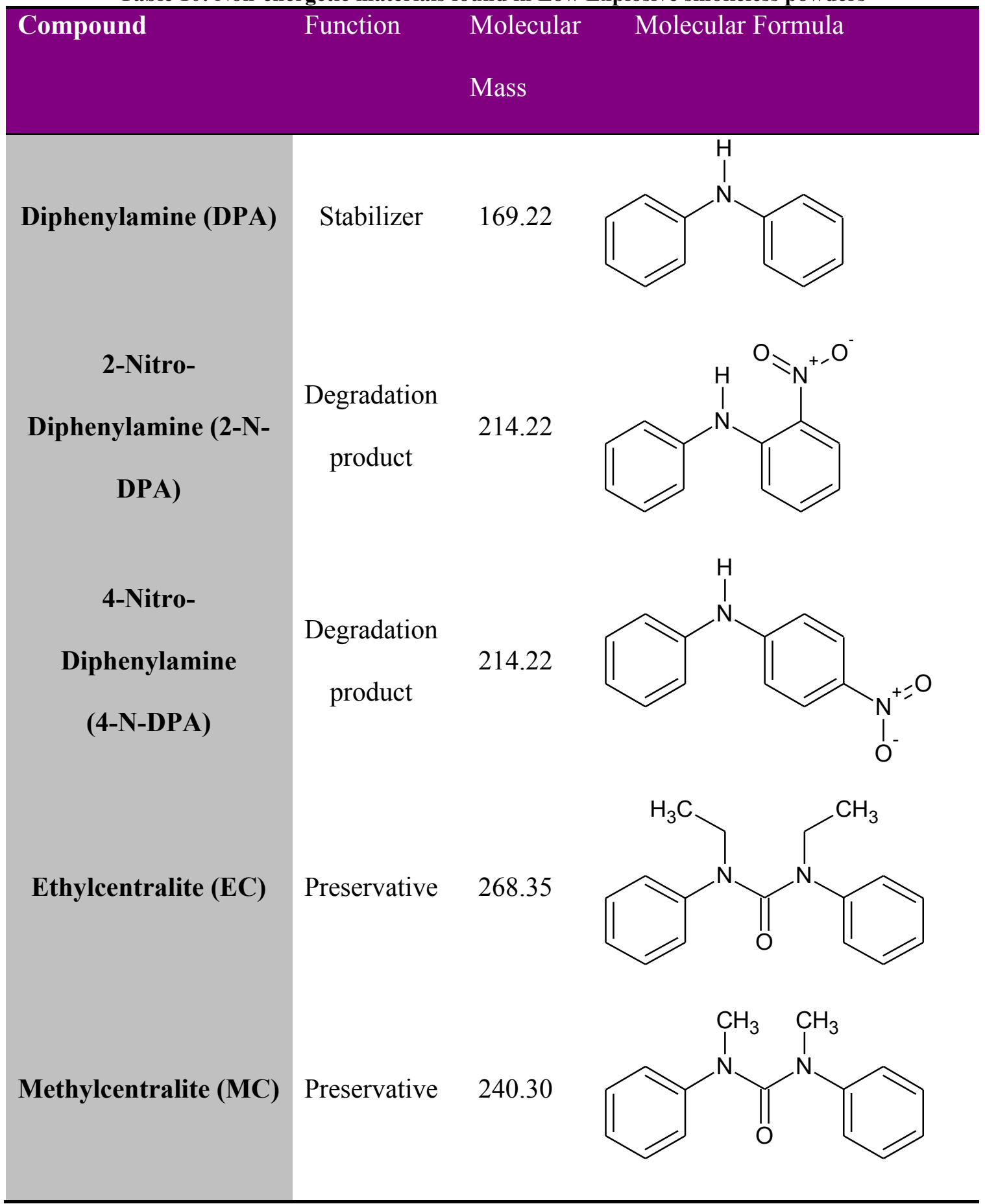




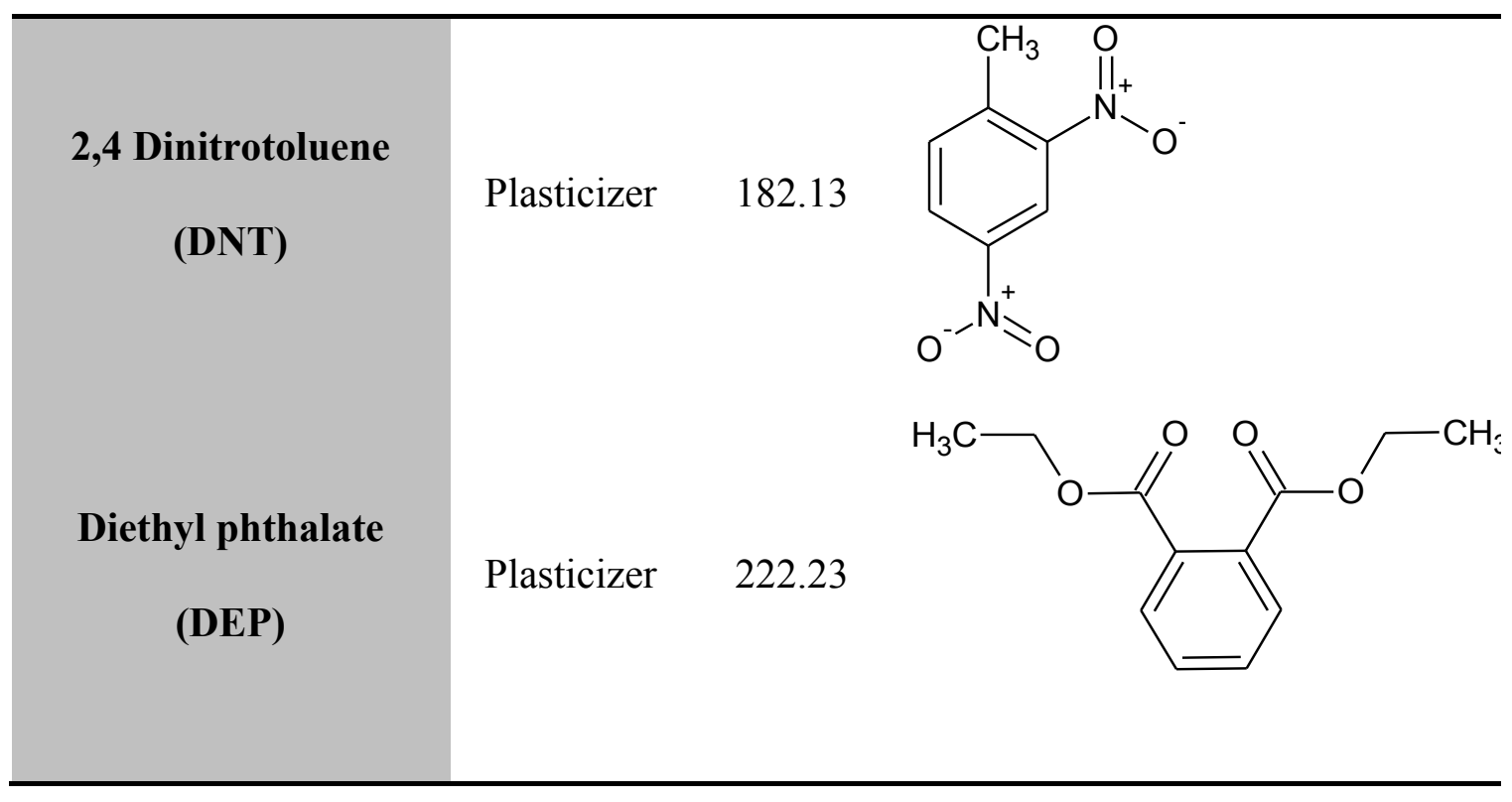

A combination of being able to detect all or some of these compounds as listed in Table 9 or Table 10 will result in not only the detection of an explosive but also the possible identification of the type of explosive as is the case for smokeless powders which have a multitude of different manufacturers that differentiate their brand of powder by customizing the formulation of additives. This final step of discriminating samples has been a long standing limitation for ion mobility, which has traditionally been used to identify a single analyte that would indicate the presence of an explosive. This has limited the utilization of Ion mobility for true analytical analysis of an explosive to determine the composition of a device. However, the utilization of an electrospray ionization source (ESI) will enhance the detection of these substances over the traditional ${ }^{63} \mathrm{Ni}$ based ion mobility devices previously used. This is achieved as a result of the much lower operating temperature of the ESI source (room temperature) than that of the traditional ${ }^{63} \mathrm{Ni}$ ionization sources (excess of $250^{\circ} \mathrm{C}$ ). As ${ }^{63} \mathrm{Ni}$ sources are used to ionize analytes desorbed in the gas phase, heat is required to maintain the analytes in a gaseous 
form, this required heat will damage thermally labile compounds found in explosives. ESI does not require the application of heat and may be referred to as a cool ionization source, allowing the ionization of these analytes without degradation under extremely mild conditions. The chemical nature of many explosives results in them being negatively ionized. As a result, many of the analyses for these thermally labile explosives compounds on the IMS are done in the negative ionization mode. Throughout reported literature, negative mode ionization is not fully understood, however attempts have been made to interpret the ions responsible for ionization in the negative mode as $\mathrm{O}_{2}-(32 \mathrm{~m} / \mathrm{z})$, $\left(\mathrm{H}_{2} \mathrm{O}\right)_{2} \mathrm{OH}-(53 \mathrm{~m} / \mathrm{z}) \mathrm{NO}_{2}-(46 \mathrm{~m} / \mathrm{z})$ as reported by Spangler et al. in 1975 [155] or CN(26m/z), CNO-(42m/z), O- $\left(\mathrm{H}_{2} \mathrm{O}\right)_{3}$ and $\mathrm{CO}_{3}-(60 \mathrm{~m} / \mathrm{z})$ by Timothy Carr in $1977[156]$. To the author's knowledge, there has been no update or general consensus to these approximate mass configurations when conducting ionization in the negative mode [3] coupled with the fact that these values still do not correspond to current negative mode ions observed from solvents analyzed by ESI-IMS. Compounding this is the complex and still incomprehensible mass assignments obtained when analyzing analytes. A few of these spectra will be shown with best guess approximations to the actual molecular structure. One of the earlier works that dates back two decades by Straub et al. attempted to decipher these ions formed in the negative mode utilizing various electrospray solvents and stated that "Solution chemistry plays a major role in negative ion chemistry" [84]. Despite these unknowns, negative ion chemistry does work and is able to effectively detect explosive compounds. In order for compounds to "explode" they must burn or combust. Combustion involves the reaction of a fuel and oxidizing agent to produce heat and gas. The simultaneous production of heat and gas creates a pressure wave that is able 
to induce great mechanical damage once released. Quite simply combustion is defined as (Equation 16):

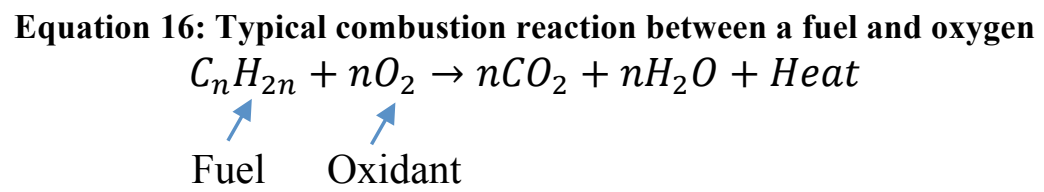

As this reaction is exothermic, an additional product along with $\mathrm{CO}_{2}$ and $\mathrm{H}_{2} \mathrm{O}$ is heat. For explosives, the reaction occurs at a much faster rate and produces greater amounts of heat. This is as a result of the explosive compound possessing the oxidant on the same molecule as the fuel (Table 9). The close proximity of the oxidant as well as the nature of this chemical bond ensures that the explosive always has sufficient oxidant in order to react, thereby creating a much more efficient reaction that produces much larger quantities of gases and heat. Consider specifically the explosive nitroglycerin (Nitroglycerin, NG).

\section{Equation 17: Combustion reaction of nitroglycerin during detonation $\mathrm{C}_{3} \mathrm{H}_{5}\left(\mathrm{NO}_{3}\right)_{3} \rightarrow 3 \mathrm{CO}_{2}+2.5 \mathrm{H}_{2} \mathrm{O}+1.5 \mathrm{~N}_{2}+0.25 \mathrm{O}_{2}$ $\uparrow$ \\ Fuel/Oxidant}

Note that this reaction takes place without oxygen as a reactant (Equation 17). Instead the oxygen is chemically bonded to the compound in the form of a nitrate $\left(\mathrm{NO}_{3}\right)$ group. This nitrate group is electronegative and as a result is amenable for detection in the negative ion mode. These oxygen rich electronegative groups may be found in any and all explosive compounds which in turn results in their highly energetic reactions known as detonation or deflagration. In addition to these oxygen rich groups, explosives also possess a carbon structure (fuel) that also determines their explosive characteristics i.e. 
whether they are nitro-aromatics, nitro-amines, peroxides or nitrate-esters. The RA4100 operation settings for explosive analysis involved the drift tube being maintained at a temperature of $150^{\circ} \mathrm{C}$, experiments on the effect of the drift tube temperature on analytes did reveal that higher temperatures $\left(170^{\circ} \mathrm{C}\right)$ provided greater signal intensity for analytes, however this higher temperature caused peaks to migrate at a very high rate, causing overlap of some analytes resulting from increased migration times (Figure 97).

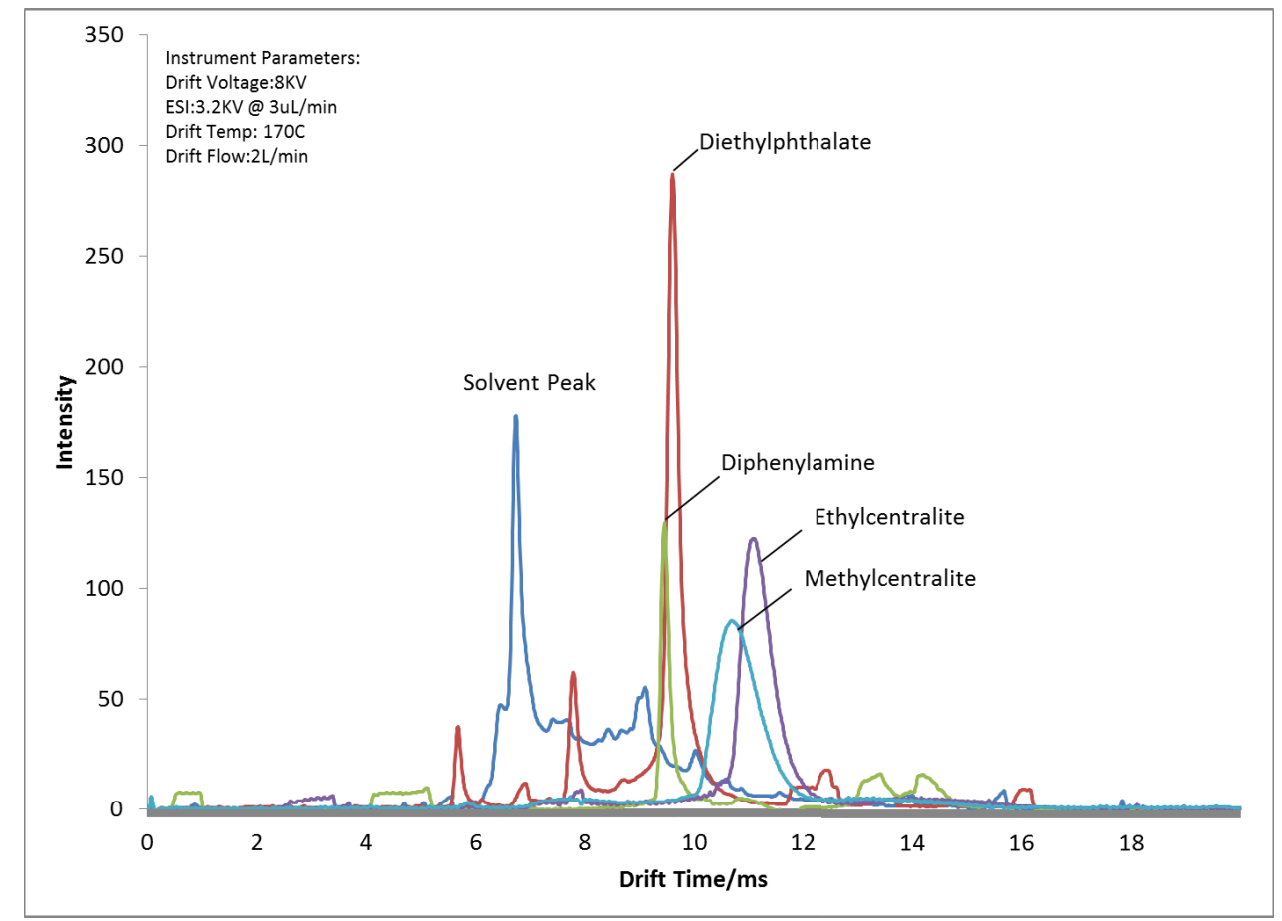

Figure 97: Analysis of DEP, DPA, MC and $\mathrm{EC}$ at $170^{\circ} \mathrm{C}$ in positive in mode

The use of very low temperatures $\left(110^{\circ} \mathrm{C}\right)$ only allowed ionization of some analytes tested, resulting in an inability to detect all targeted compounds. At $110^{\circ} \mathrm{C}$ only Diethyl phthalate (DEP) was detected while diphenylamine (DPA), Methylcentralite (MC) and Ethylcentralte (EC) were neither ionized nor detected at this temperature (Figure 98). 


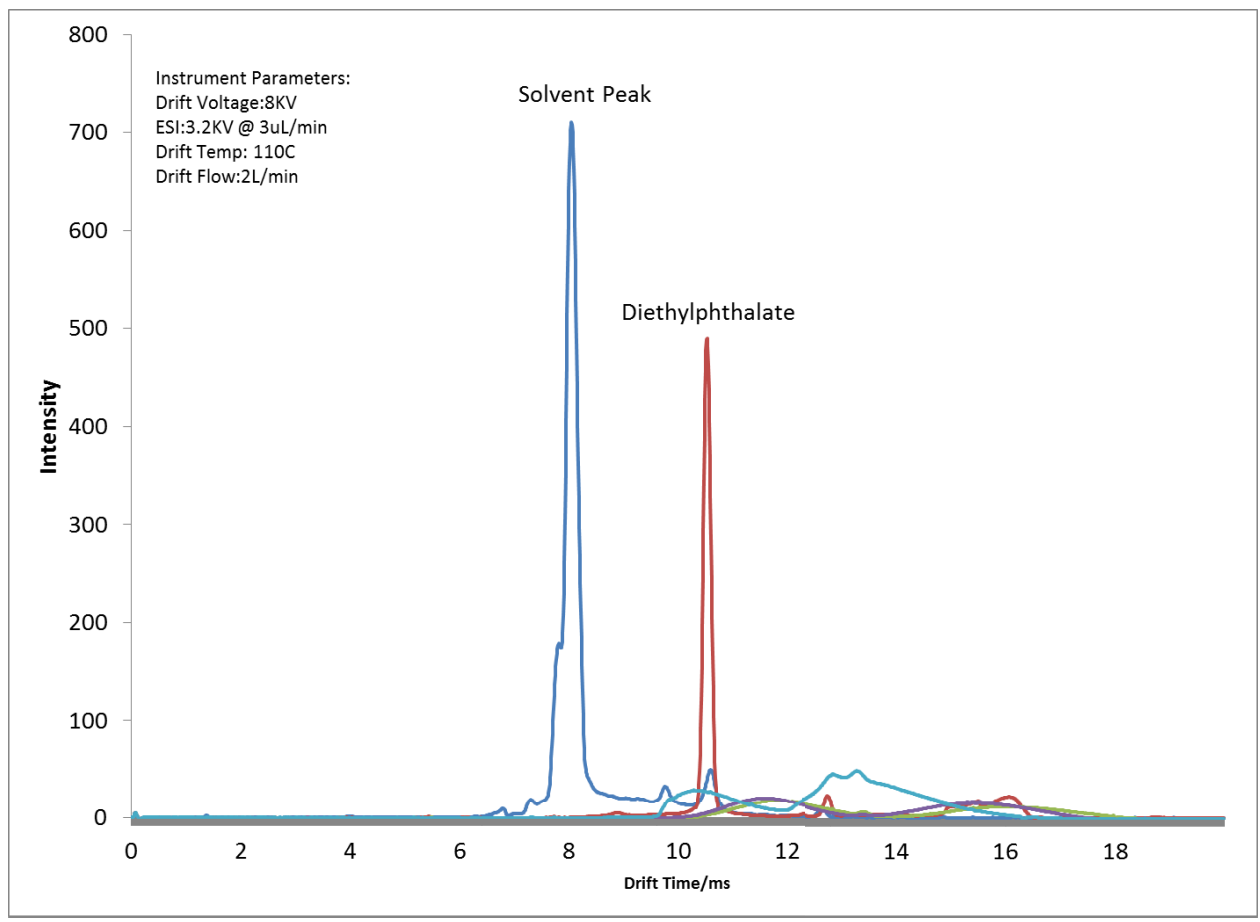

Figure 98: Analysis of DEP, DPA, MC and $\mathrm{EC}$ at $110^{\circ} \mathrm{C}$ in positive in mode, showing inability to detect DPA, MC and EC at this temperature.

However, the median temperature of $150^{\circ} \mathrm{C}$ provided the best compromise of signal intensity, detection capabilities of all analytes and sufficient resolution between analytes (Figure 99). 


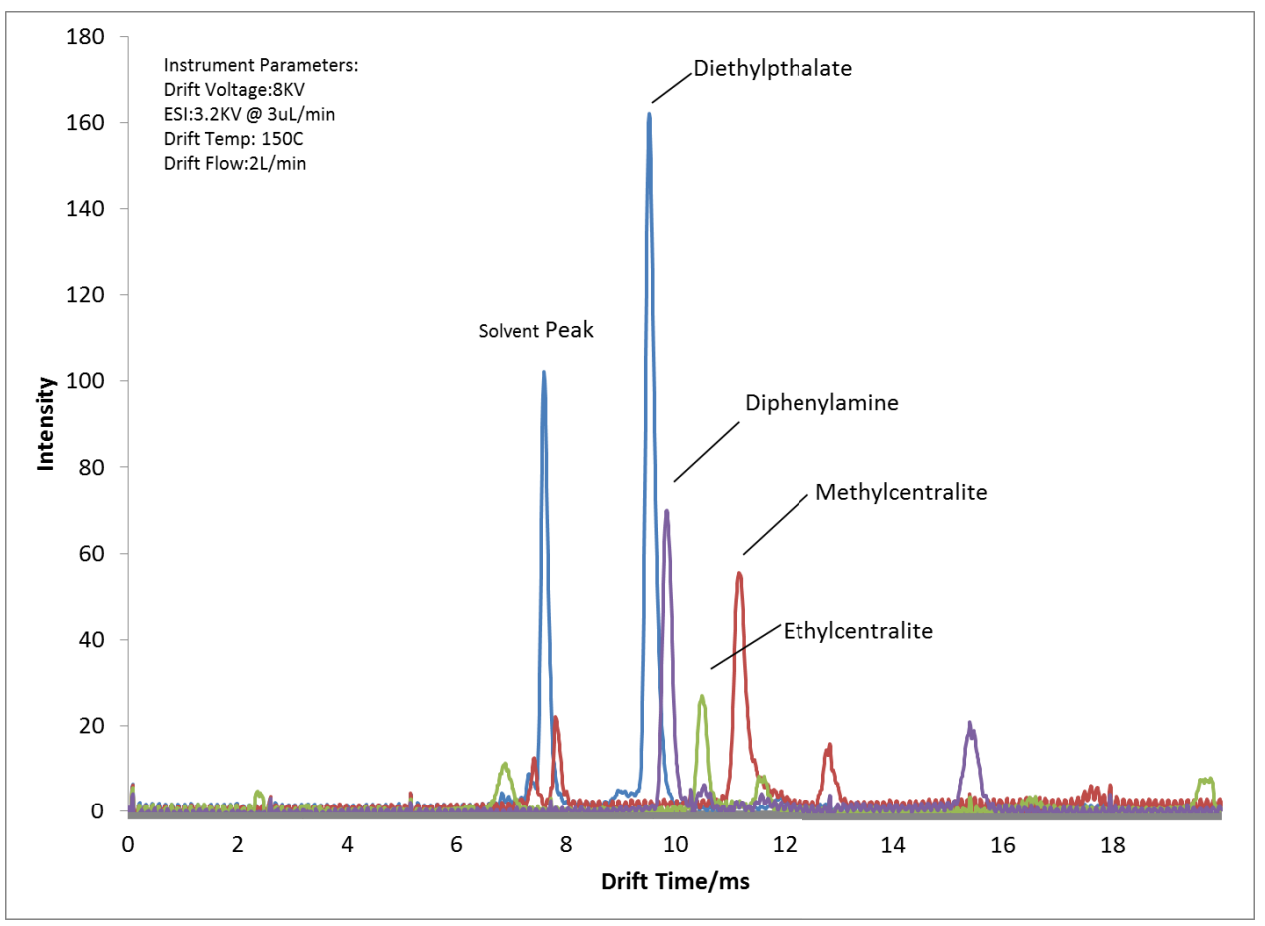

Figure 99: Analysis of DEP, DPA, MC and EC at $150 \mathrm{C}$ in positive in mode showing the detection of all analytes with good separation.

Single ion monitoring of the expected molecular ion masses from these analytes resulted in a clear indication of the drift time for each analyte within the ion mobility spectrum (Figure 100). As can be seen from the results in Figure 100, DPA, DEP, MC and EC are all detected in the positive mode, forming protonated analytes. The optimized solvent chemistry used for these analyses was adopted from the section Analysis of Multiple Component Mixtures Utilizing ESI-IMS-MS (pg. 98) Analysis and provided good ionization and separation characteristics. 


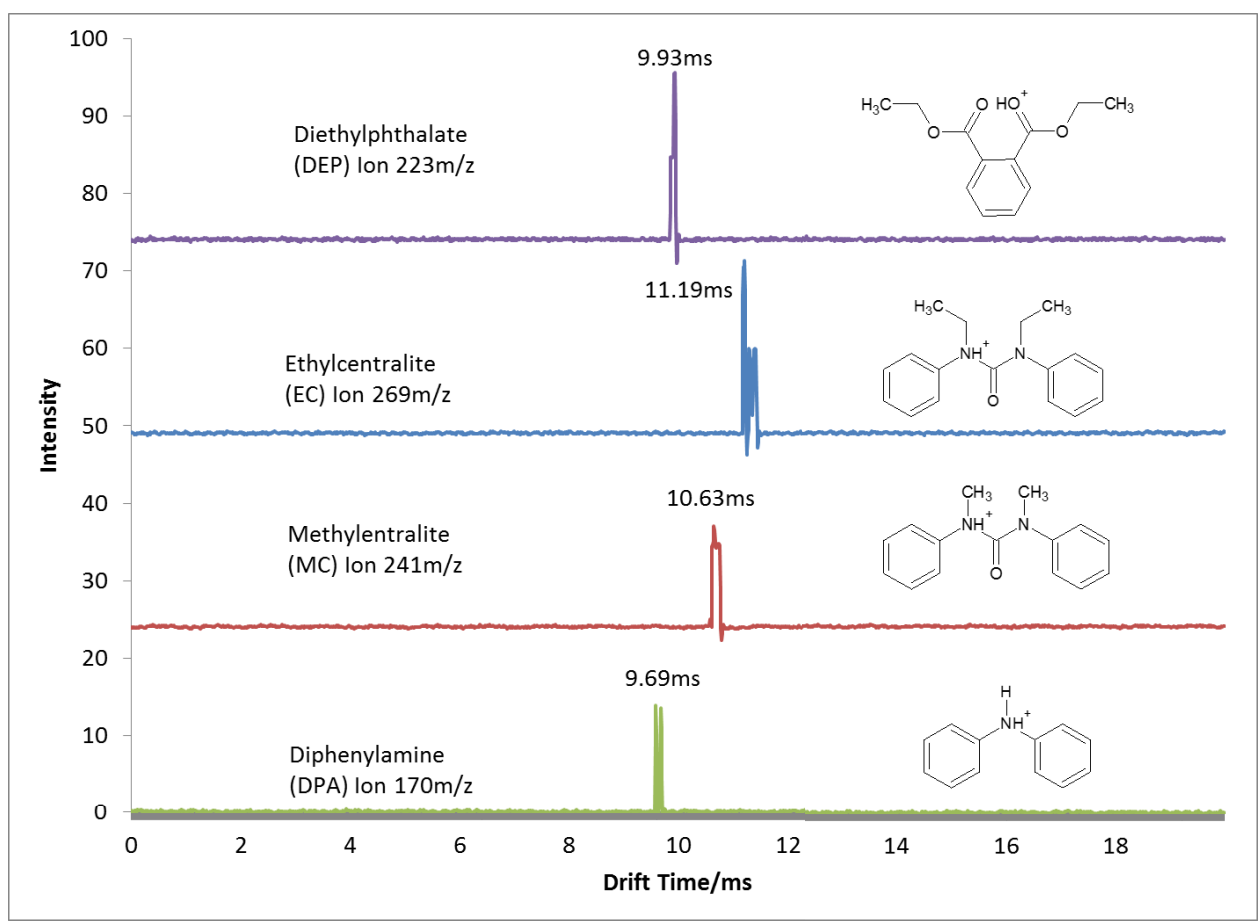

Figure 100: Single Ion Monitoring of molecular ions of DEP, DPA, MC and EC at 150C in positive in mode.

The remaining analytes from Table 10 DNT, NG, 2-NDPA and 4-NDPA were all analyzed in the negative mode. As previously stated, negative mode analysis did prove to be more challenging than analysis in the positive mode [46]. In addition to adjustments in drift tube temperature, the drift gas itself was varied in order to improve results. It was found that the use of nitrogen as a drift gas in the negative mode often provided less noisy spectra and reduced the chances of arcing from the electrospray ionization source (Figure 101). 


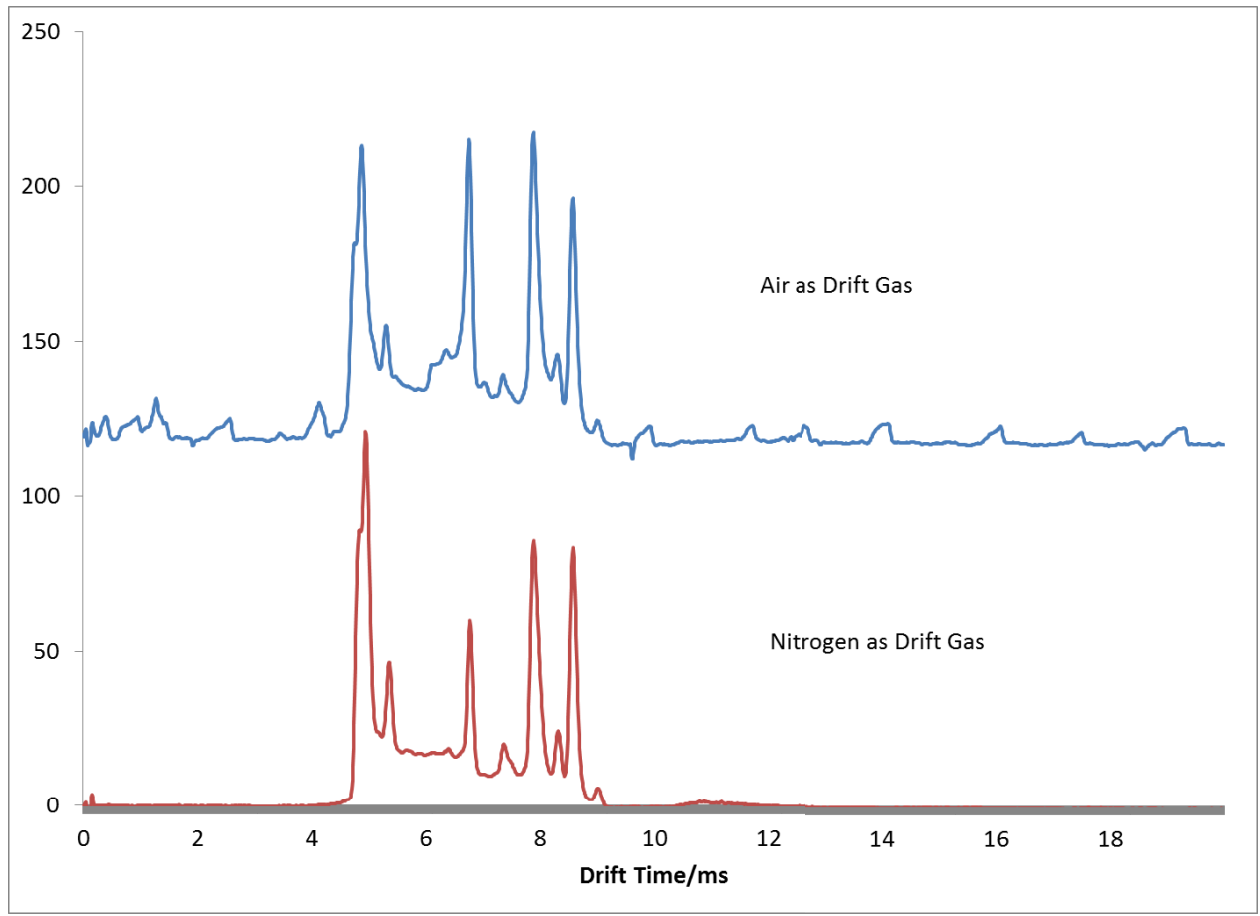

Figure 101: Comparison of Standards DNT, NG, 2-NDPA and 4-NDPA in negative mode using Air vs. Nitrogen drift gas.

This was believed to be the result of the nitrogen acting as an electron scavenging gas that inhibits corona discharge and thus increases the stability of the electrospray $[81 ; 84]$. Again, optimized solvent chemistry for the negative mode was used for these experiments as outlined in section Analysis of Multiple Component Mixtures Utilizing ESI-IMS-MS (pg. 98). This included an 80:20 Methanol:Water mixture with 0.1\% Chloroform. The mass spectra for these analytes were compared and single ion monitoring performed to determine the type of ionic species being detected in relation to the ion mobility spectra (Figure 102). Of interest was the fact that both 2-NDPA and 4NDPA produced the proton abstracted species $(\mathrm{M}-\mathrm{H})^{-}$as well as the chloride adducted species $(\mathrm{M}+\mathrm{Cl})^{-}$in the chlorinated solvent system. The detection of nitroglycerin proved difficult, with the analyte having very poor ionization response. It was also observed that 
the nitroglycerin fragmented producing the nitrate ion at mass to charge $62 \mathrm{~m} / \mathrm{z}$ versus a molecular adducted or proton abstracted ion.

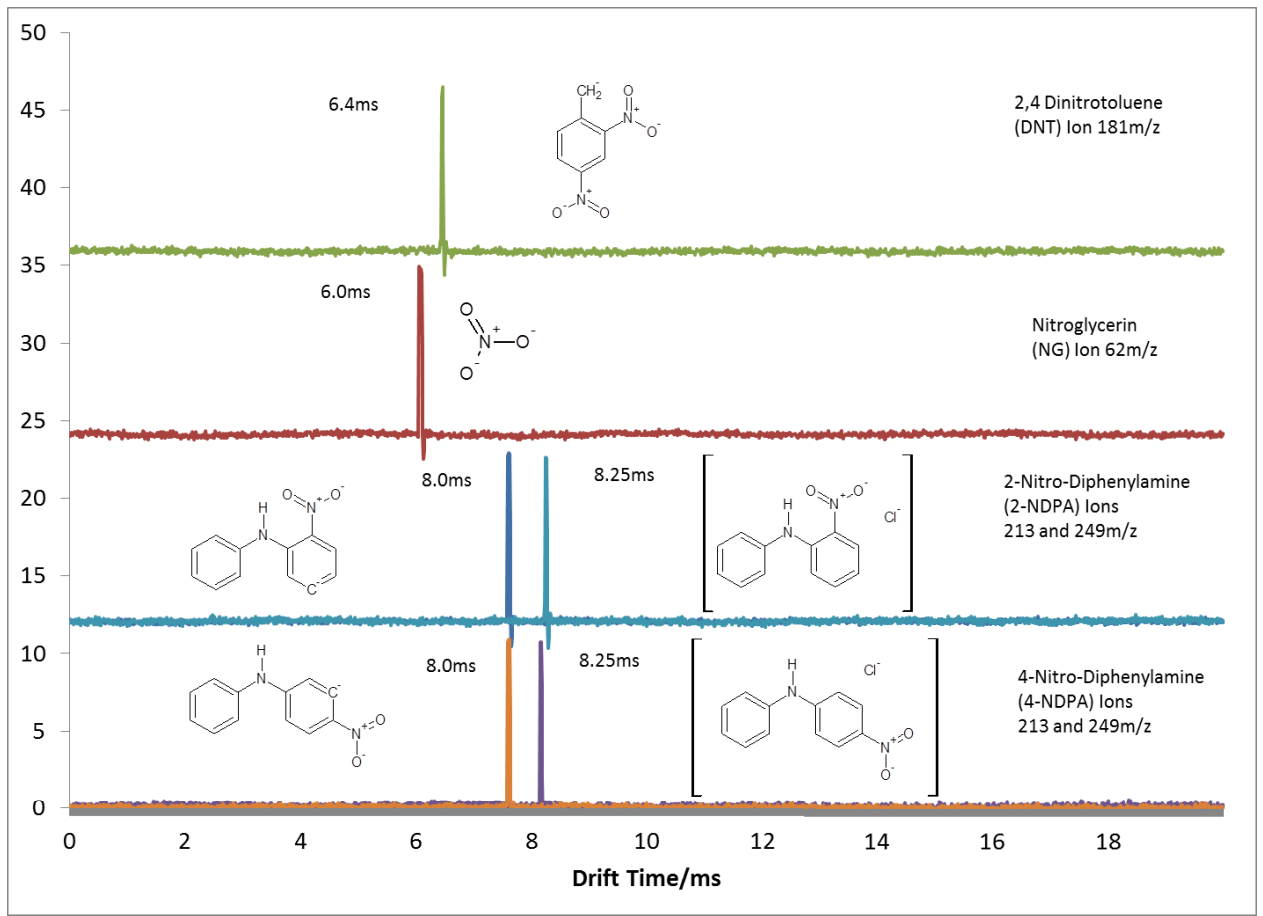

Figure 102: Single Ion Monitoring of ions of DNT, NG, 2-NDPA and 4-NDPA at $150^{\circ} \mathrm{C}$ in negative in mode.

Dinitrotoluene (DNT) produced the proton abstracted species regardless of the solvent system in which it was dissolved. With both the solvent system determined for optimal ionization of analytes in both the positive and negative mode and the ionic species for analytes determined, emphasis shifted to the preparation of analytes extracted from actual samples in order for them to be analyzed. 


\subsubsection{Analysis of Smokeless Powder Samples}

Smokeless powders are produced and sold as extruded cellulose based pellets that are impregnated with the active ingredients listed in Table 10 as well as the explosive nitroglycerin from the list in Table 9. As a result of the production process, the target ingredient must first be extracted from the pellets before being detected by the analytical system of choice. An extraction scheme was thus adopted and developed from the previous work of Mathis et al. [82;157]. This extraction scheme involved an initial step of measuring $20 \mathrm{mg}$ gravimetrically of the smokeless powder and dissolving with $1 \mathrm{ml}$ of

dichloromethane, this was left to stand for one hour. This was followed by evaporation of an aliquot of the supernatant $(300 \mu 1)$ with nitrogen gas. The residue was then re-dissolved in the appropriate solvent determined by the method of analysis whether positive mode or negative mode analysis as shown in Figure 103. A filtration step was added using a $0.2 \mu \mathrm{m}$ pore PTFE filter to prevent undissolved residue from entering and clogging the capillary plumbing of the ESI source. 


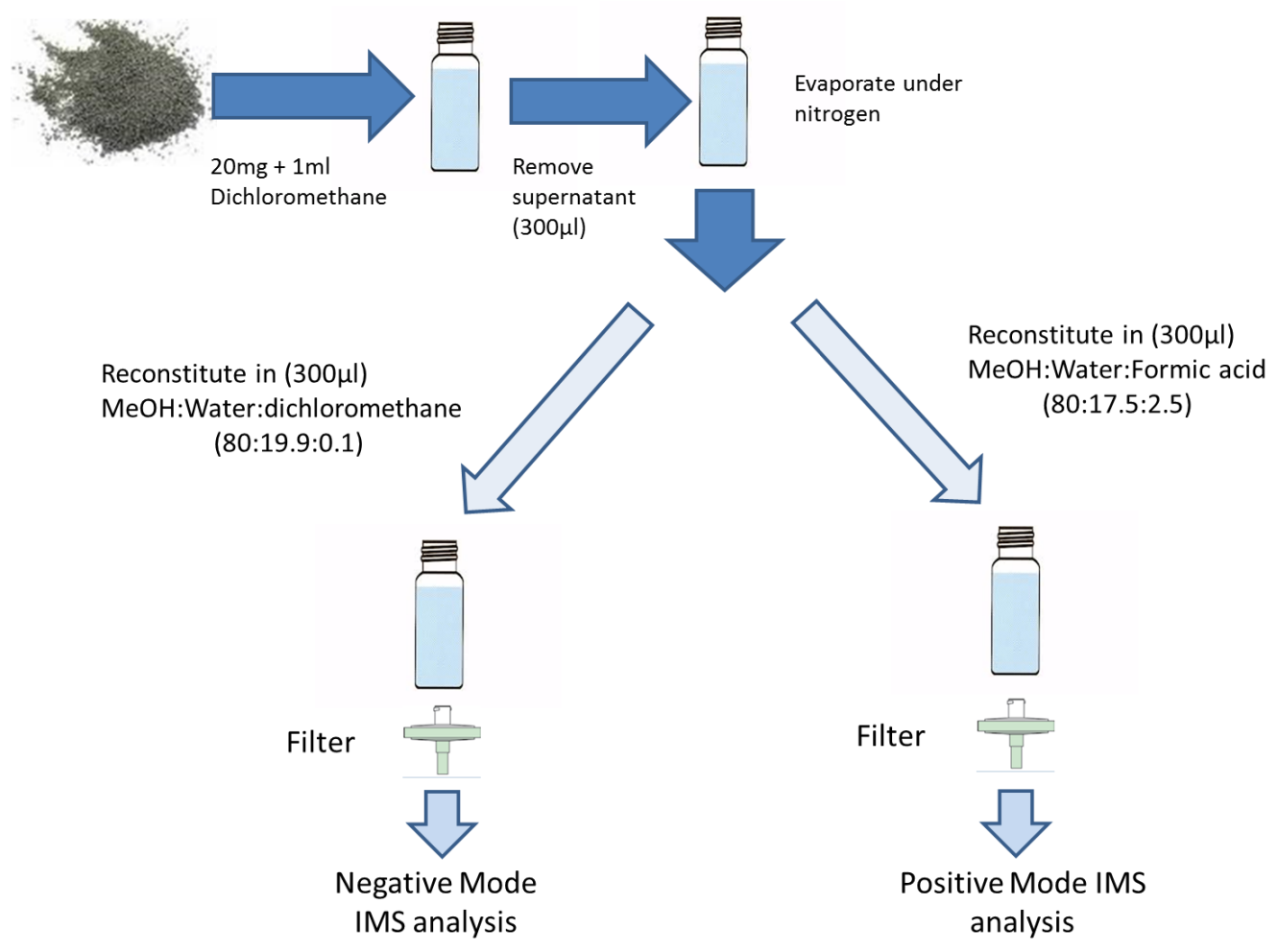

Figure 103: Extraction scheme used for Smokeless powder analysis by ESI-IMS-MS

This extraction scheme was used to separate target analytes from solid smokeless powder samples in preparation for analysis by ESI-IMS-MS. In optimizing the extraction time for the interaction between the solid smokeless powder and dichloromethane extraction solvent, a variety of extraction times were tested and these samples analyzed utilizing GC-ECD. It was observed that after an extraction time of one hour, there was only slight enrichment of analytes within the solvent compared to an extended extraction time of up to 96 hours (Figure 104). In an effort to conserve time, the shortest extraction time was selected of one hour. 


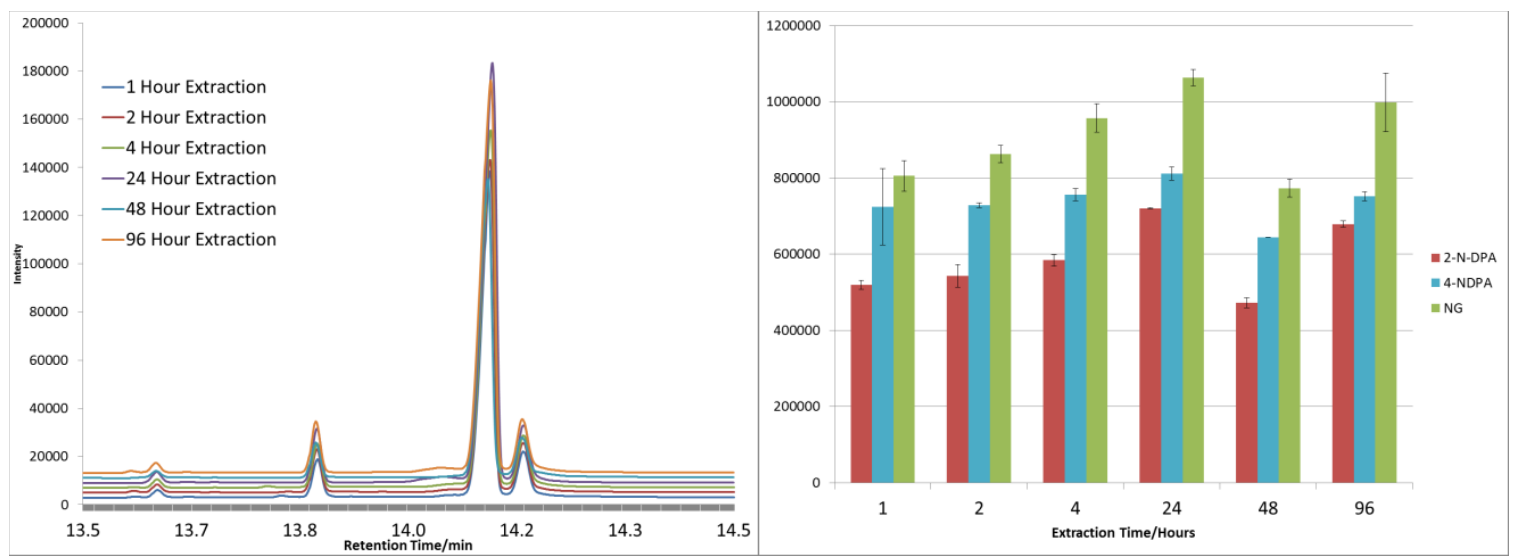

Figure 104: GC-ECD response of smokeless powder extracts with varying extraction time.

The optimized extraction procedure was used to analyze thirty six smokeless powder samples from 5 different brands, Vihta Vouri, Accurate Arms, Hodgdon, IMR and Alliant (Red and Green Dot). Within these five brands were also different lots of smokeless powders, seventeen in all. Samples were treated as indicated by Figure 103. The analysis was conducted not just to identify the presence of target compounds listed in Table 10 but also to possibly discriminate between different brands of smokeless powders based on the non-energetic additives detected. This final step will be essential in enhancing the tools required by law enforcement during times of mass disasters when the presence and origin of a suspected or detonated devices is crucial. Such disasters often overwhelm law enforcement with the sheer number of samples requiring analysis, ESI-IMS-MS offers a much faster method of analysis compared to the current gold standard of gas chromatographic analysis. Case in point, the 2005 Bali bombings where over 5000 shrapnel fragments were recovered for analysis, these analyses were done rapidly using a mobile IMS lab in order to determine the type of explosive used [158]. IMS provides analysis of these compounds in milliseconds (Figure 105) while traditional chromatographic analysis of these same compounds takes several minutes as shown in 
Figure 104. A summary of the typical results obtained from these analysis are shown below in Figure 105.

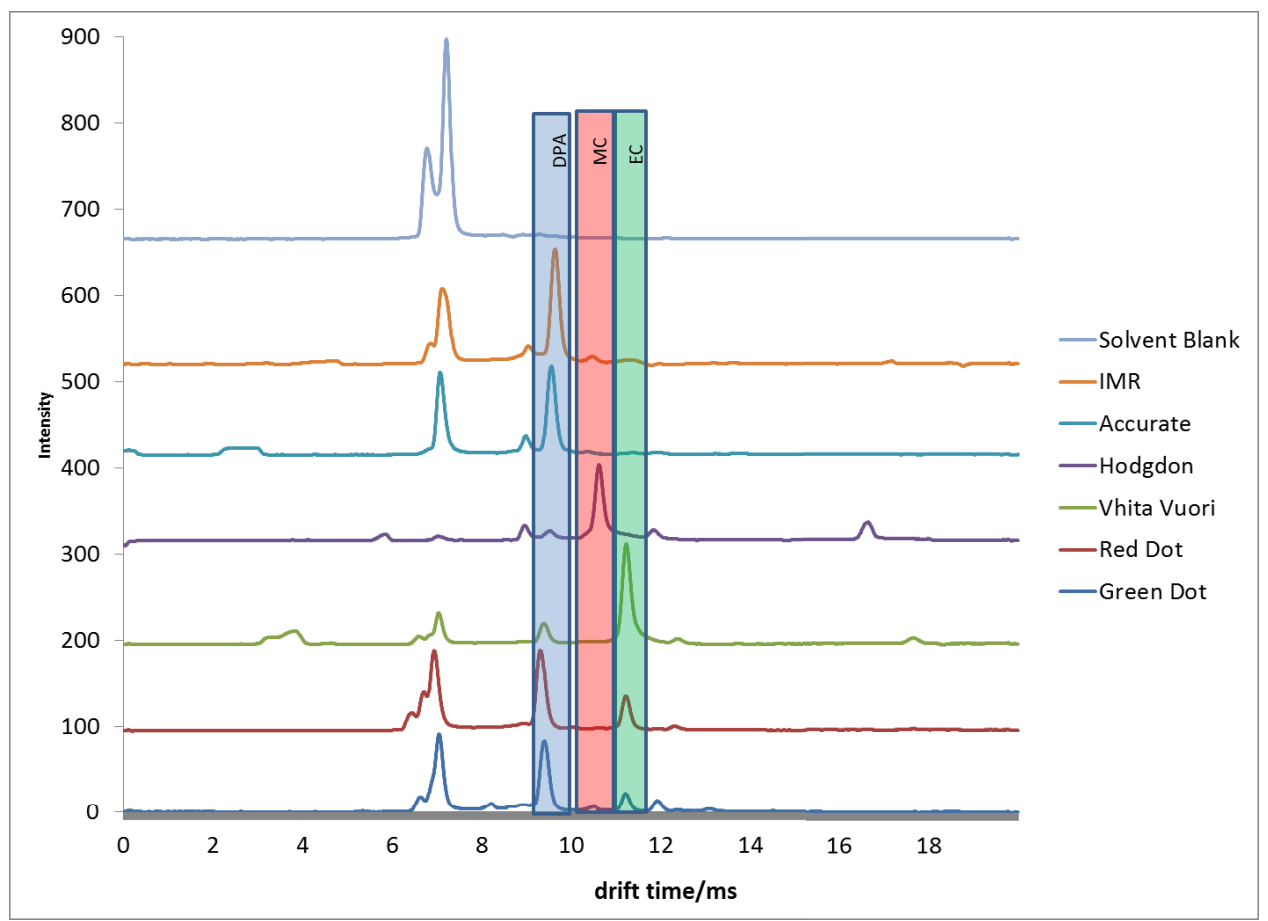

Figure 105: Analysis of smokeless powder extracts in positive ion mode

It is crucial to note here, that the results obtained from these extracts would not be possible without having the optimized solution chemistry outlined in section (Analysis of Multiple Component Mixtures Utilizing ESI-IMS-MS, pg. 98), as without it, competitive ionization would reveal only one ion from each sample greatly reducing the discriminating power of the technique. The spectra show the detection of three analytes, DPA, MC and EC which differ across the smokeless powder samples. A full table containing these results is shown in Table 11. 
Table 11: Results from ESI-IMS-MS analysis of Smokeless powder extracts in positive ion mode ( 1 indicates the presence of the compound)

\begin{tabular}{|c|c|c|c|c|c|c|}
\hline Ref\# & Manufacturer & Lot Number & DPA & DEP & EC & MC \\
\hline 113 & Hodgdon & HS-6 & 1 & 1 & 0 & 0 \\
\hline 164 & Accurate & S744 & 1 & 1 & 1 & 0 \\
\hline 165 & Hodgdon & HS-7 & 1 & 1 & 1 & 0 \\
\hline 241 & IMR & SR-7625 & 1 & 1 & 0 & 0 \\
\hline 275 & Accurate & 4350 & 1 & 1 & 1 & 0 \\
\hline 657 & VhitaVouri & $24 N 41$ & 0 & 1 & 1 & 0 \\
\hline 751 & Accurate & No.2 & 0 & 0 & 0 & 0 \\
\hline 754 & Alliant Red Dot & 987 & 0 & 1 & 1 & $\underline{0}$ \\
\hline 755 & Alliant Bulls eye & & 0 & 0 & 1 & 0 \\
\hline 756 & Alliant Blue Dot & 296 & 0 & 1 & 1 & 0 \\
\hline 757 & Alliant Red Dot & 995 & 0 & 1 & 1 & 0 \\
\hline 758 & Alliant Red Dot & 992 & 0 & 1 & 1 & 0 \\
\hline 759 & Alliant & 2400 & 0 & 1 & 1 & 0 \\
\hline 760 & Alliant & Herco & 0 & 1 & 1 & 0 \\
\hline 761 & Alliant Reloader & 15 & 0 & 0 & 1 & 0 \\
\hline 762 & Alliant Green Dot & 765 & 0 & 1 & 1 & 0 \\
\hline 763 & Alliant Green Dot & 783 & 1 & 1 & 1 & 0 \\
\hline 764 & Alliant Unique & 577 & 0 & 0 & 1 & $\underline{0}$ \\
\hline 765 & Alliant American Select & & 1 & 1 & 1 & 0 \\
\hline 766 & Alliant Power Pistol & & 0 & 0 & 1 & $\underline{0}$ \\
\hline 768 & Hodgdon & $B L-C(2)$ & 0 & 0 & 0 & 0 \\
\hline 771 & IMR & 3031 & 1 & 1 & 1 & 0 \\
\hline 772 & IMR & Hi Skol 700x & 0 & 0 & 1 & 0 \\
\hline 773 & Hodgdon & Longshot & 0 & 0 & 0 & 1 \\
\hline 776 & Hodgdon & H414 & 1 & 1 & 0 & $\underline{0}$ \\
\hline 777 & Hodgdon & $\mathrm{H} 4831$ & 1 & 1 & 0 & 0 \\
\hline 779 & Hodgdon & Retumbo & 1 & 1 & 0 & 0 \\
\hline 780 & Hodgdon & Titewad & 0 & 1 & 0 & $\underline{0}$ \\
\hline 781 & Hodgdon & Benchmark & 1 & 1 & 0 & 0 \\
\hline 783 & Hodgdon & International Clays & 1 & 1 & 0 & $\underline{0}$ \\
\hline 784 & Hodgdon & $\mathrm{H} 48315 \mathrm{C}$ & 1 & 1 & 0 & $\underline{0}$ \\
\hline 785 & Hodgdon & $\mathrm{H} 380$ & 0 & 0 & 0 & 0 \\
\hline 786 & Hodgdon & $\mathrm{H} 110$ & 0 & 0 & 1 & 0 \\
\hline 787 & Hodgdon & Lil gun & 0 & 0 & 0 & 0 \\
\hline 788 & Hodgdon & H322 & 1 & 1 & 0 & 0 \\
\hline NA & Alliant Red dot & 987 & 0 & 0 & 1 & 0 \\
\hline
\end{tabular}

From Table 11, it can be concluded that each brand of smokeless powder had a

characteristic analyte composition. By creating a dendrogram with this data utilizing JMP 
version 10 statistical software, it was revealed that there is sufficient discriminating power to classify these smokeless powders by brand (Figure 106). The roots of the branches stemming from the same brand of powders converge at points that are more similar than roots of branches from different brands of powders.

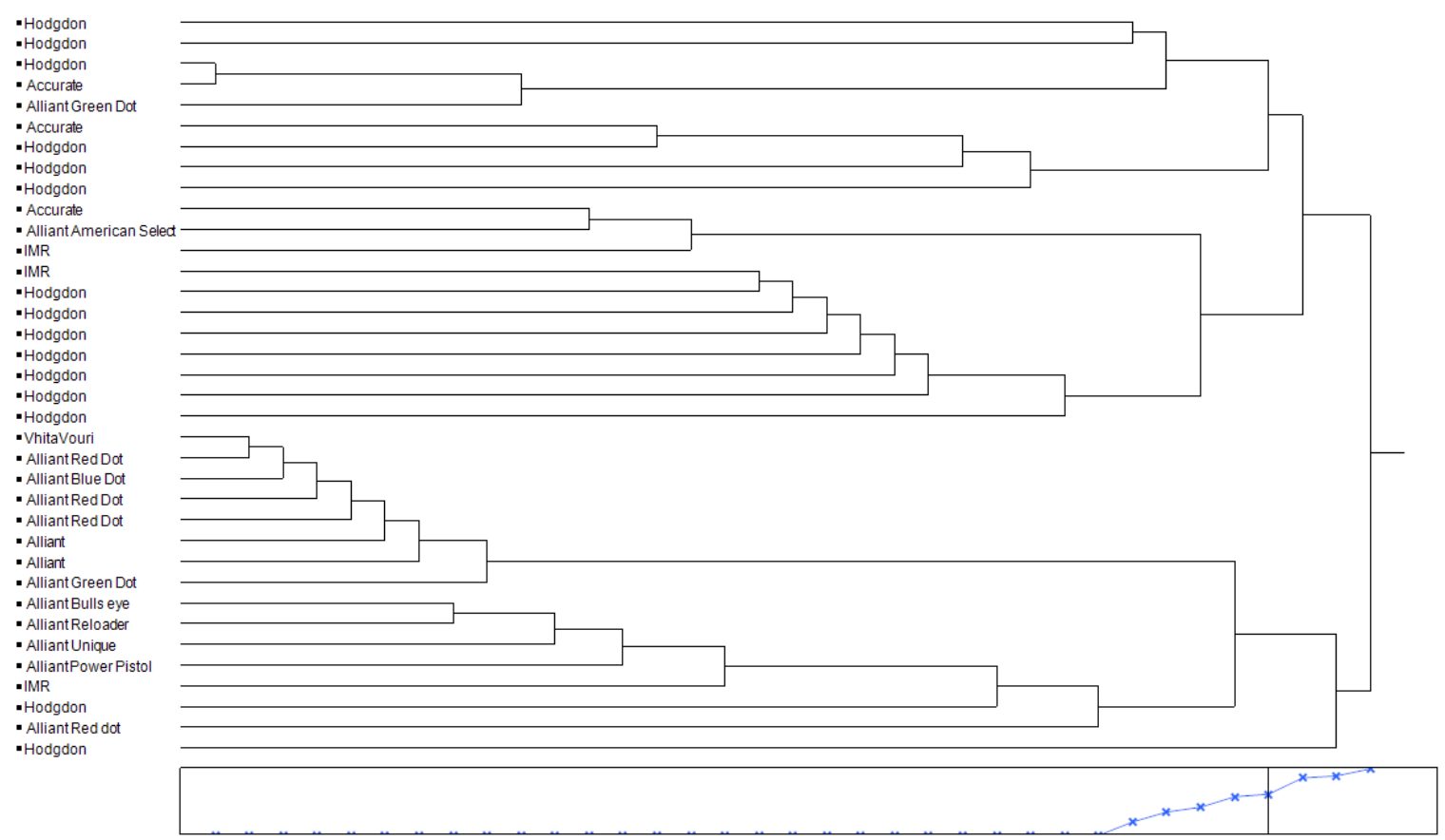

Figure 106: Dendrogram showing clustering of smokeless powders based on analysis by ESI-IMS-MS in positive ion mode.

This verifies that the RA4100 is capable of not only detecting the analytes that indicate the presence of smokeless powders, but is also able to detect multiple analytes from a single sample thereby allowing discrimination of these smokeless powder samples.

To obtain additional powers of discrimination, powders were also analyzed in the negative ion mode. This allowed for the detection of the other components such as NG, DNT and 2 and 4 N-DPA. It should be noted that the analytes 2 and 4 N-DPA could not be discriminated utilizing the ESI-IMS-MS technique as they possessed identical drift 
times and identical mass to charge ratios. However, by cross referencing these samples with GC-MS data that was also performed, it was discovered that only two of the thirty six powders analyzed contained both 2 and 4-N-DPA. Most powders contained only 2-NDPA, therefore removing the ambiguity when detecting this analyte from these powders when conducting ESI-IMS-MS analysis. The negative mode results are displayed and summarized in Table 12 below. Table 12 shows that most of these powders contain nitroglycerin (NG) as the analyte detected. This is expected as nitroglycerin is the main explosive component used to manufacture smokeless powders. What was not expected however was the inability to detect the other components of these powders while in the negative mode. This inability to multiplex in the negative mode was believed to be as a result of the extremely high levels of nitroglycerin found within each sample. This high level of nitroglycerin suppressed the ionization of other analytes while in the negative mode during electrospray ionization, despite the best efforts of the optimized solvent chemistry to compensate for this. 
Table 12: Results from ESI-IMS-MS analysis of Smokeless powder extracts in negative ion mode (1 indicates the presence of the compound)

\begin{tabular}{|c|c|c|c|c|c|}
\hline Ref\# & Manufacturer & Lot Number & DNT & 2 or 4 NDPA & NG \\
\hline 113 & Hodgdon & HS-6 & 0 & 0 & 1 \\
\hline 164 & Accurate & $S 744$ & 0 & 1 & 1 \\
\hline 165 & Hodgdon & HS-7 & 0 & 0 & 1 \\
\hline 241 & IMR & SR-7625 & 0 & 1 & 0 \\
\hline 275 & Accurate & 4350 & 1 & 0 & 0 \\
\hline 657 & VhitaVouri & $24 N 41$ & 0 & 0 & 0 \\
\hline 751 & Accurate & No. 2 & & 1 & 1 \\
\hline 754 & Alliant Red Dot & 987 & 0 & 0 & 1 \\
\hline 755 & Alliant Bulls eye & & 0 & 0 & 1 \\
\hline 756 & Alliant Blue Dot & 296 & 0 & 0 & 1 \\
\hline 757 & Alliant Red Dot & 995 & 0 & 0 & 1 \\
\hline 758 & Alliant Red Dot & 992 & 0 & 0 & 0 \\
\hline 759 & Alliant & 2400 & 0 & 0 & 1 \\
\hline 760 & Alliant & Herco & 0 & 0 & 1 \\
\hline 761 & Alliant Reloader & 15 & 0 & 0 & 1 \\
\hline 762 & Alliant Green Dot & 765 & 0 & 0 & 1 \\
\hline 763 & Alliant Green Dot & 783 & 0 & 0 & 1 \\
\hline 764 & Alliant Unique & 577 & 0 & 0 & 0 \\
\hline 765 & Alliant American Select & & 0 & 1 & 1 \\
\hline 766 & Alliant Power Pistol & & 0 & 0 & 1 \\
\hline 768 & Hodgdon & $\mathrm{BL}-\mathrm{C}(2)$ & 0 & 1 & 0 \\
\hline 771 & IMR & 3031 & 0 & 0 & 1 \\
\hline 772 & IMR & Hi Skol 700x & 0 & 0 & 0 \\
\hline 773 & Hodgdon & Longshot & 0 & 0 & 1 \\
\hline 776 & Hodgdon & $\mathrm{H} 414$ & 0 & 0 & 1 \\
\hline 777 & Hodgdon & $\mathrm{H} 4831$ & 1 & 1 & 0 \\
\hline 779 & Hodgdon & Retumbo & 1 & 1 & 0 \\
\hline 780 & Hodgdon & Titewad & 0 & 0 & 1 \\
\hline 781 & Hodgdon & Benchmark & 1 & 0 & 0 \\
\hline 783 & Hodgdon & International Clays & 0 & 1 & 1 \\
\hline 784 & Hodgdon & $\mathrm{H} 48315 \mathrm{C}$ & 1 & 1 & 0 \\
\hline 785 & Hodgdon & H380 & 0 & 1 & 1 \\
\hline 786 & Hodgdon & $\mathrm{H} 110$ & 0 & 0 & 1 \\
\hline 787 & Hodgdon & Lil gun & 0 & 0 & 1 \\
\hline 788 & Hodgdon & H322 & 1 & 0 & 0 \\
\hline NA & Alliant Red dot & 987 & 0 & 1 & 0 \\
\hline
\end{tabular}


A statistical clustering model was used to classify the smokeless powders analyzed by brand based on the data obtained from the analysis in the negative mode. The obtained dendrogram affirms the data from Table 12 . There is significantly more overlap of brands when using the data from the negative ion mode as seen in Figure 107 versus the much more distinct clustering of powders by brand when using the data from the positive ion mode data as seen in Figure 106.

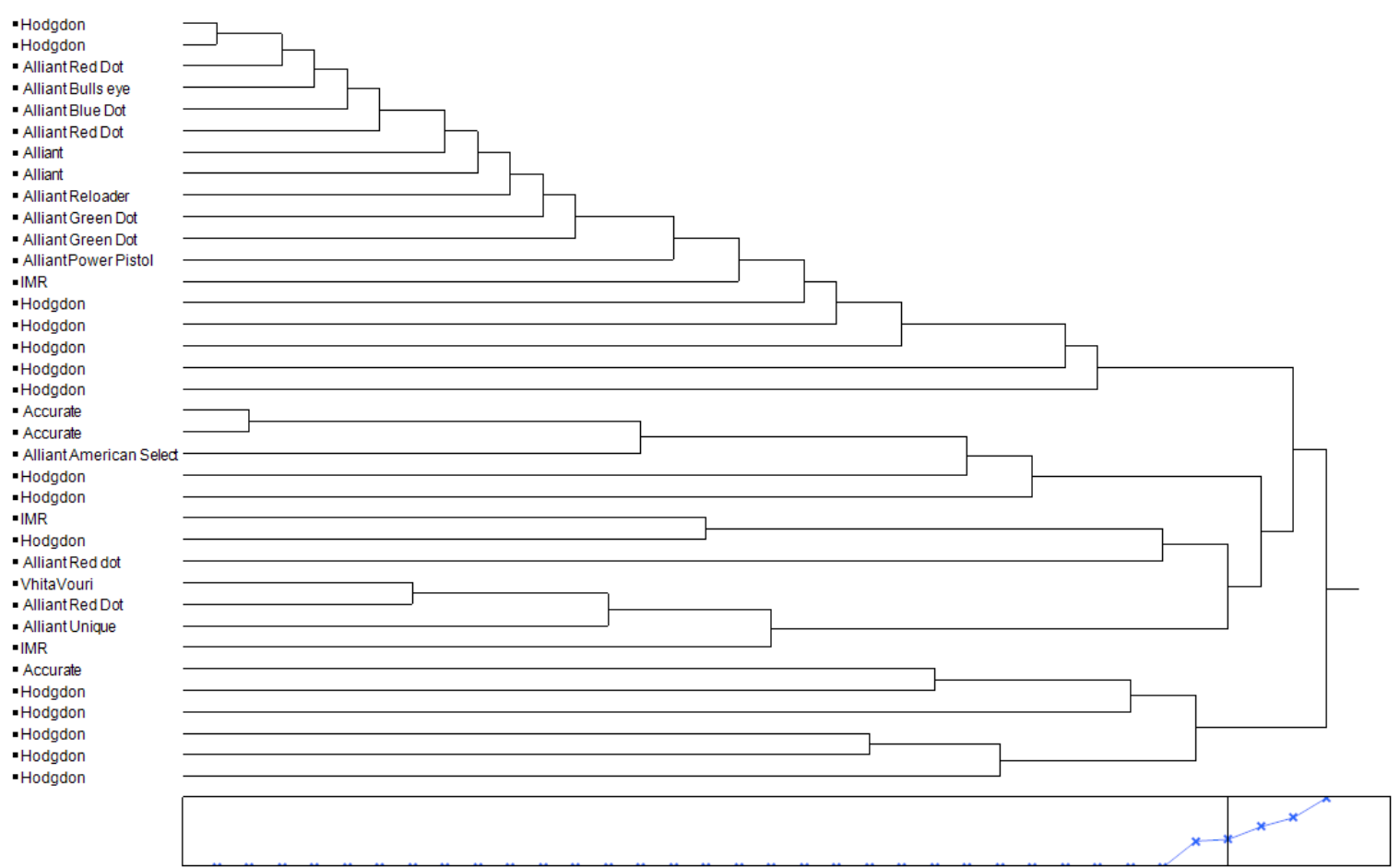

Figure 107: Dendrogram showing clustering of smokeless powders based on analysis by ESI-IMS-MS in negative ion mode

The result of this difference in discriminating power can be clearly seen from the type of data obtained when powders are analyzed in the positive mode versus the negative mode. The relatively large amounts of nitroglycerin in these powders when analyzed in the negative mode would result in a salient peak for only this analyte, suppressing the ionization of other analytes present within the sample (Figure 108). A mixture of 
components are more balanced in the positive mode, this allows the ionization solvent to promote detection of all components as shown in Figure 109.

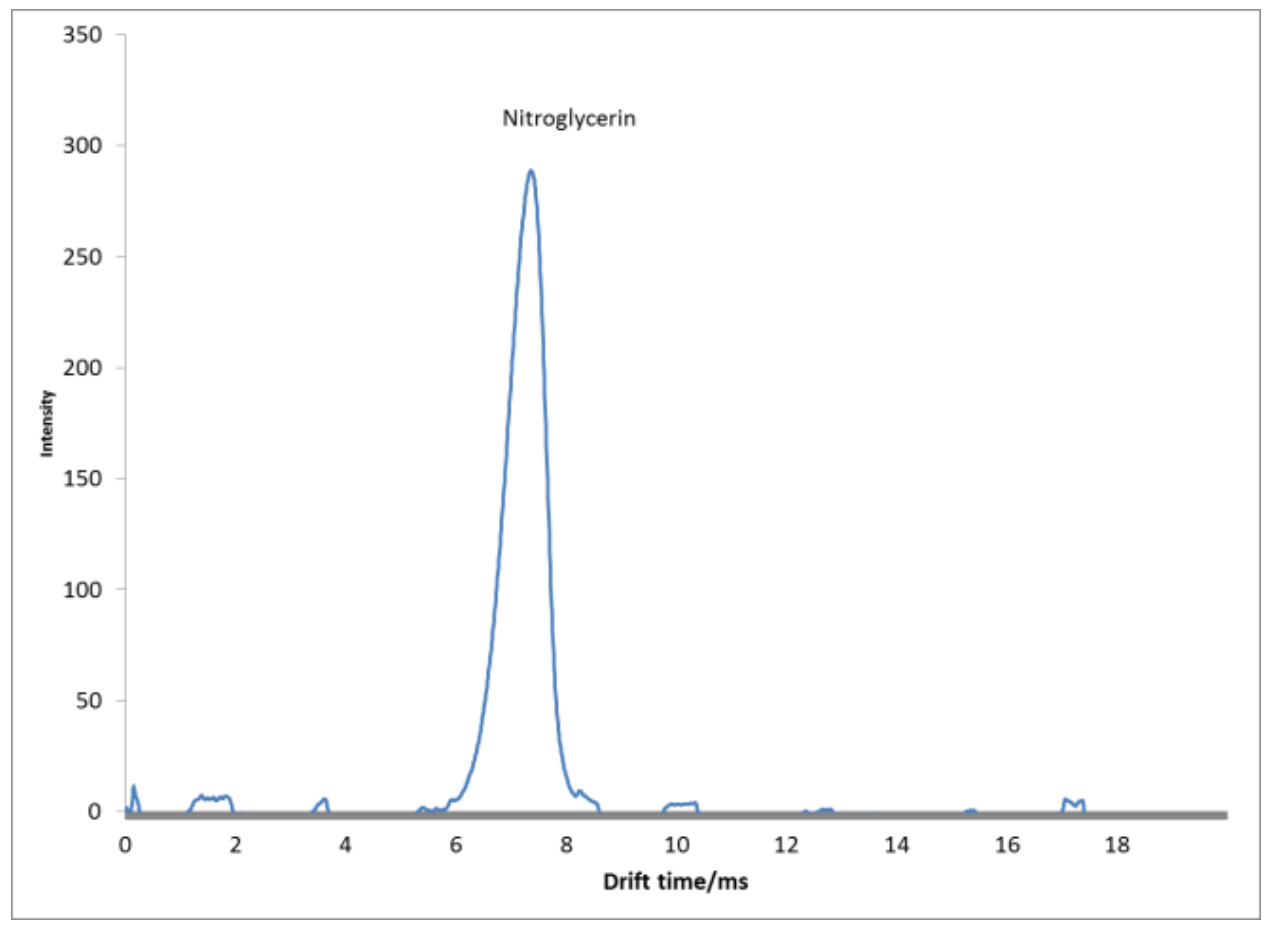

Figure 108: Analysis of Green dot in negative ion mode

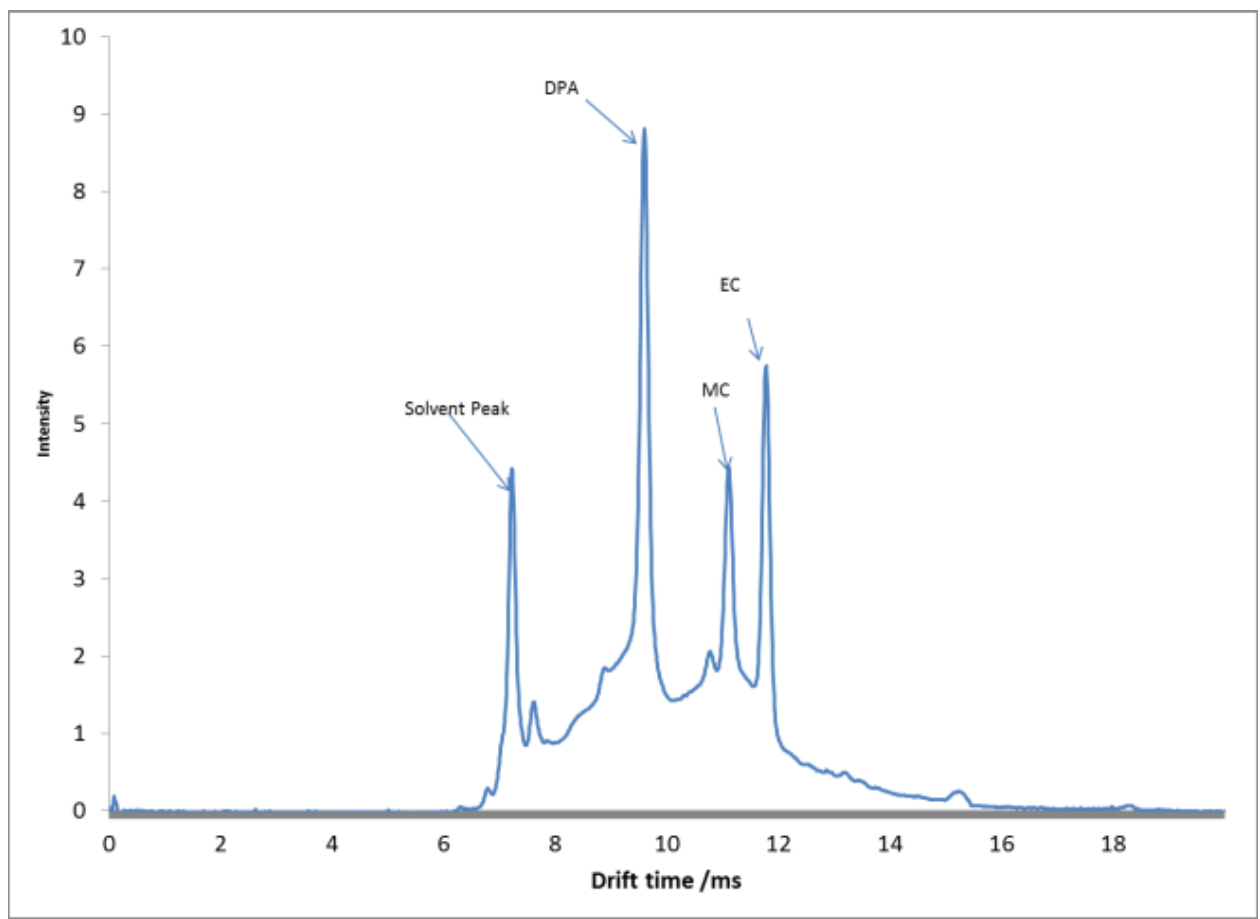

Figure 109: Analysis of Green dot in the positive ion mode 


\subsubsection{Conclusions:}

Methods were developed to allow the detection of thermally labile compounds commonly found in smokeless powders in both the positive and negative ionization mode utilizing ESI-IMS-MS. The positive ionization mode provided more discriminating power when analyzing these samples, allowing samples to be classified by brand. Though the limitation of ionization competition observed when conducting analysis in the negative mode was not overcome through these experiments, the ability to detect and discriminate these powders from their analytes in the positive ion mode is encouraging. This will be a powerful tool for law enforcement for pre and post blast studies where identification of numerous fragments of evidence in a timely manner as well as the ability to identify the type of explosive by brand. The more information that can be generated from evidence increases the chances of law enforcement in identifying and eventually prosecuting a suspect. The current method indicates a leap forward in forensic analysis of explosives samples such as smokeless powders, by allowing analysis and characterization of a sample in only $20 \mathrm{~ms}$, versus the current fastest reported analysis time of 8 minutes for similar analytes utilizing LC-MS [159]. 
4.6 Detection and Mass Spectrometric Characterization of explosive analytes using ESI-IMS-MS

Though the non-energetic materials were extensively targeted for analysis by ESI-IMSMS, other high energetic materials as identified in Table 9 were analyzed. These compounds were analyzed only as standard reference materials, since their high energetic nature is accompanied by stricter controllers and more limited access than their low energy or non-energetic counterparts listed in Table 10 The compounds analyzed included RDX, HMX, TATP, HMTD, TNT and PETN. These were all analyzed with various solvent systems to determine optimal signal intensity as well as to determine the type of ionic species derived and detected by the mass spectrometer.

\subsubsection{RDX and HMX}

RDX or Research Department eXplosive has the IUPAC name 1,3,5-Trinitroperhydro1,3,5-triazine (RMM $222.12 \mathrm{~g} / \mathrm{mol})$. This compound is a nitro-amine and as expected by high explosives has numerous nitrite groups chemically bonded to its structure that comprises of a six membered ring with three carbons substituted with nitrogens along its structure. The Nitrite groups are bound to these nitrogens as shown in 
Figure 110a in order to promote efficient and rapid combustion (detonation). HMX or High Molecular weight rdX (RMM $296.15 \mathrm{~g} / \mathrm{mol}$ ), is also a nitro-amine that has a similar structure to RDX with numerous nitrite groups attached to an eight membered ring. This ring also has carbon atoms substituted with nitrogen atoms as shown in Figure 110b.
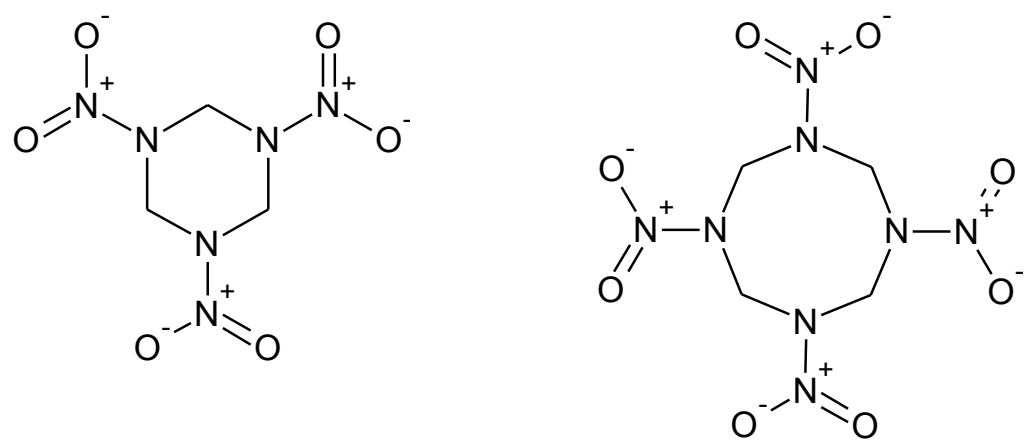

(a)

Figure 110: Molecular structure of (a) RDX and (b) HMX

Both these analytes are among the most powerful high explosives available and are used primarily by the military, though other more powerful explosives are currently being developed [160]. Traditional methods of analysis for these compounds have been difficult as a result of the low melting point of $276^{\circ} \mathrm{C}$ and $205^{\circ} \mathrm{C}$ for HMX and RDX respectively. This normally results in decomposition of the analytes prior to detection by gas chromatographic techniques which are the most common methods of detection $[16 ; 30 ; 95 ; 161]$. Of all the compounds analyzed, RDX proved to be one of the most difficult to detect. Due to the structure of RDX, it does not form stable proton abstracted ions $(\mathrm{M}-\mathrm{H})^{-}$in the negative mode and so gives poor response when sprayed in $\mathrm{MeOH}: \mathrm{H}_{2} \mathrm{O}$ solution using ESI. This made characterizing any observed ions difficult. 
Characterization was done by utilizing relatively high concentrations of the analyte as can be seen from the IMS spectra of 200ng/ $\mu \mathrm{L}$ of $\mathrm{RDX}$ in $\mathrm{MeOH}: \mathrm{H}_{2} \mathrm{O}$ (Figure 111), there is a dominant peak at $7.78 \mathrm{~ms}$.

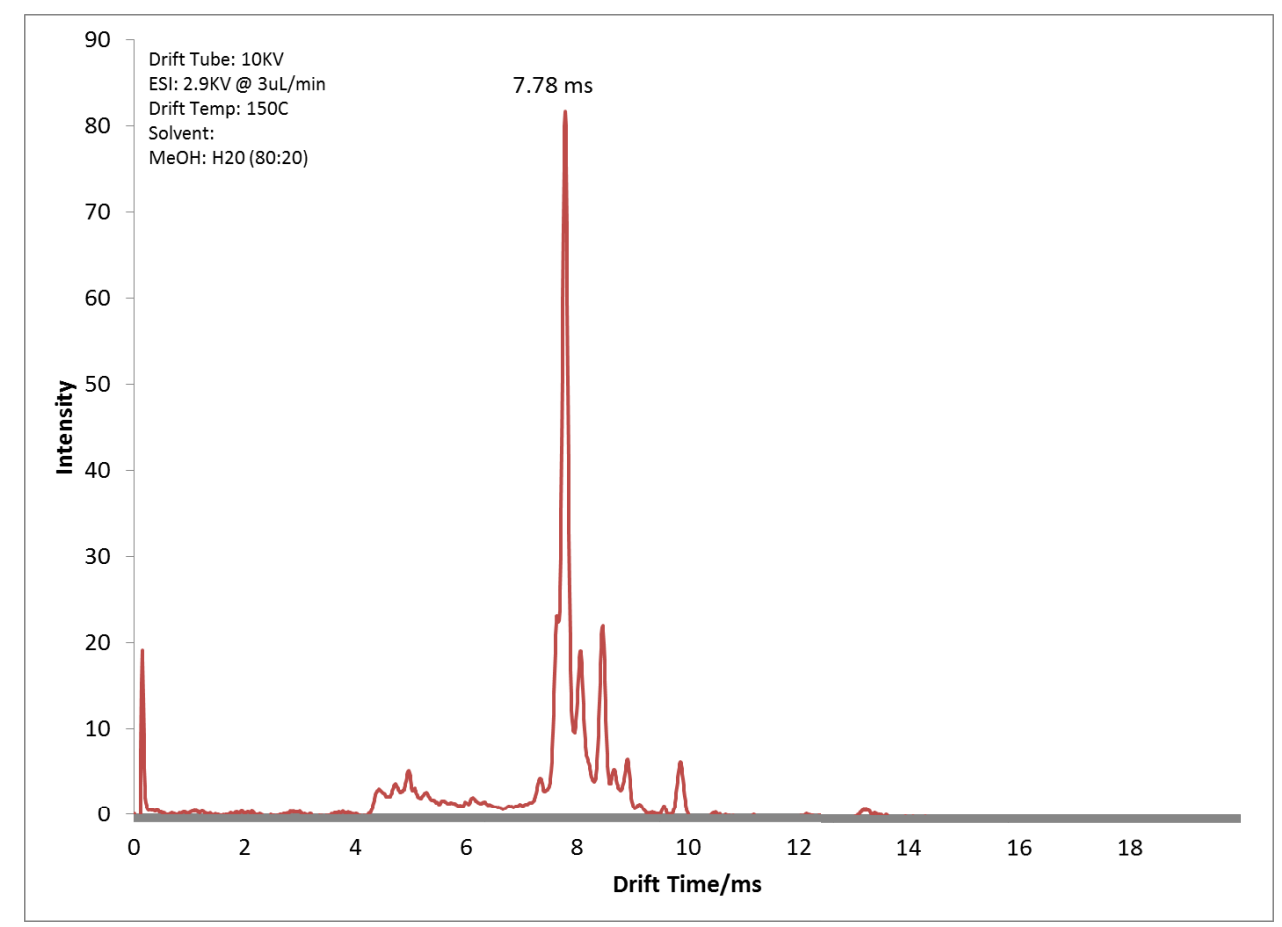

Figure 111: 200ng/ $\mu \mathrm{L}$ of RDX in MeOH:Water analyzed in the negative ion mode.

The corresponding mass spectrum for this analyte shows a mass to charge ratio of 267 . This mass to charge ratio of RDX has been previously reported in the literature as the molecule $\left[\mathrm{M}+\mathrm{NO}_{2}-\mathrm{H}\right]^{-}$[32]. However, this apparent structure for this molecule is thermodynamically unlikely and the more thermodynamically likely structure is $\left[\mathrm{M}+\mathrm{NO}_{2}\right]^{-}$with a corresponding mass to charge of 268 which was not observed (Figure 112). 


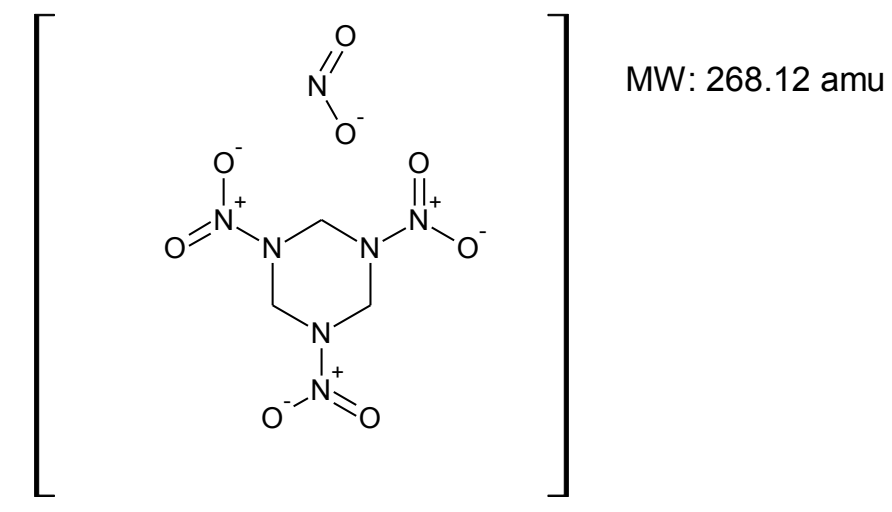

Figure 112: Thermodynamically favorable adduct of RDX not observed in ESI-IMS-MS analysis in negative mode.

The nitrite adduct show in Figure 112 is suspected to be formed from cleavage of another RDX molecule or possibly from the electrospray solvent that forms numerous nitrite and nitrate molecules in combination with air while creating gas phase ions. Regardless of the process, this expected molecule is not observed in the negative mode when using solvents that contain only Methanol and Water. A molecular structure for RDX analyzed by ESIIMS has been reported by Tam at el and includes an excited radical molecule of RDX with an adducted nitrite anion [162]. The analysis of HMX followed suit with the results obtained for RDX. An ion of $\mathrm{m} / \mathrm{z} 341$ was obtained in the mass spectrum versus an expected $342 \mathrm{~m} / \mathrm{z}$ as shown in Figure 113.

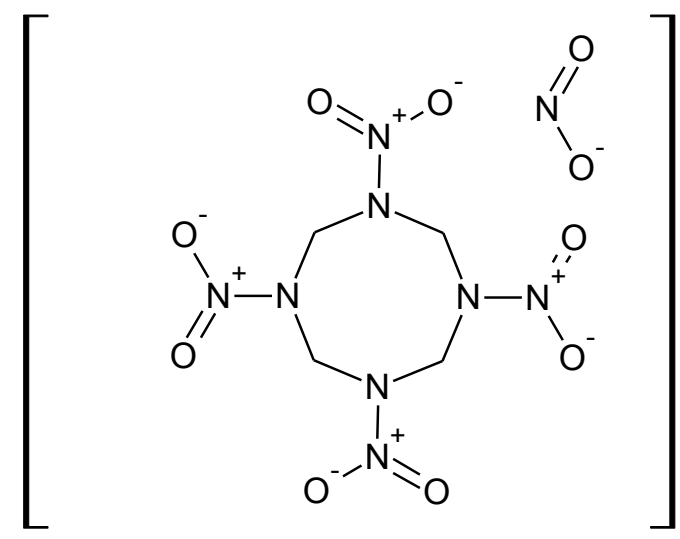

MW: $342.16 \mathrm{amu}$

Figure 113: Expected HMX adduct not observed on ESI-IMS-MS in negative ion mode 
Both these results confounded attempts at interpreting the spectra observed when conducting negative mode analysis. But it was interesting to observe that both nitroamines produced the same anomaly, i.e. producing anions in methanol and water solutions that were one amu less than the actual ions observed. However, more predictable ions were obtained when additives were placed within the electrospray solvent. The use of halides such as chlorine in the form of sodium chloride or as an organic halide such as chloroform produced strong peaks of mass to charge $257 \mathrm{~m} / \mathrm{z}$ indicating the process of a chloride adducted species for RDX or $331 \mathrm{~m} / \mathrm{z}$ indicating the chloride adducted HMX. Further improvements in response for these compounds were obtained when using solvents containing Nitrate salts. A Methanol:Water (80:20) solution with $10 \mathrm{mMol}$ Ammonium Nitrate produced very intense peaks for RDX and HMX on the IMS and corresponding ions of 358 and $267 \mathrm{~m} / \mathrm{z}$ for Nitrate adducted HMX and RDX respectively. Of interest was the fact that both HMX and RDX possessed near identical drift times of $7.8 \mathrm{~ms}$ when sprayed in a solution of the methanol and water only or methanol and water containing chloride ions. In solutions containing nitrate ions however, the observed drift times for these analytes increased slightly to $8.05 \mathrm{~ms}$ indicating a reduction in reduced mobility as this adduct forms a much heavier ion than either the suspected nitrite adducted species or the chloride adducted species.

\subsubsection{TATP and HMTD}

Triacetone triperoxide (TATP, RMM $222.24 \mathrm{~g} / \mathrm{mol}$ ) and Hexamethylenetriperoxide (HMTD, RMM $208.17 \mathrm{~g} / \mathrm{mol}$ ) stand out as explosives as they possess a different functional group that allows them to undergo rapid combustion or detonation [163-165]. 
As shown in Table 9, these compounds collectively known as peroxide based explosives, do not possess the nitrite or nitrate functional groups but instead have peroxide functional groups that easily break to react and oxidize fuels (Figure 114).

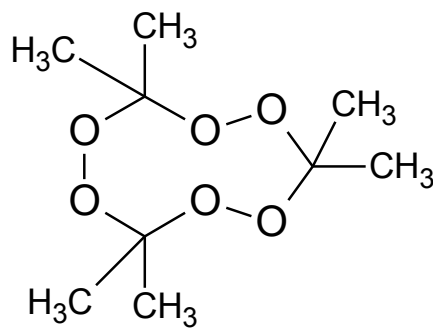

(a)

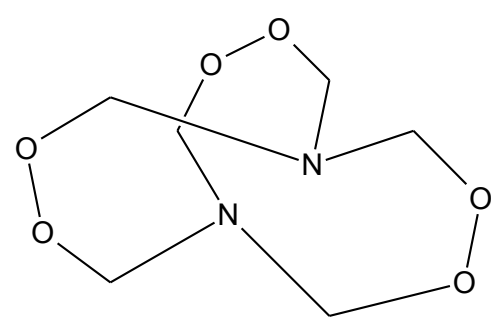

(b)

Figure 114: Molecular structure of (a) TATP and (b) HMTD

The result of these weak peroxide bonds has made TATP extremely shock sensitive, making it very unstable and not used in military applications despite its greater explosive power than TNT [166;167]. However, TATP and to a lesser extent HMTD can be made by simple acid catalyzed reactions of acetone and concentrated hydrogen peroxide, both of these starting materials are completely accessible to the public and easily obtained without any scrutiny from law enforcement [168;169]. As a result, peroxide based explosives have been manufactured and widely used for illicit activity [170]. There have been numerous incidents reported in literature where these compounds have been manufactured clandestinely and used as improvised explosive devices. The 2005 London bombings [171], attempted attack on American Airlines Flight AA63 in 2001 and the attempted Northwest Airlines Flight 253 in 2009 (Underwear bomber) are recent examples where peroxide based explosives have been used [172]. The ability to identify 
the explosive used in these cases was crucial in guiding law enforcement to prevent the further use of these substances in future incidents by unscrupulous individuals and terrorist organizations. The current chemical structure of peroxide based explosives has not only made it too unstable for military use, but also made it completely different as far as detection. The nitrogen based functional groups of other explosives cause them to be preferentially ionized in the negative ion mode; peroxide based explosives however are preferentially ionized in the positive ion mode [28;173]. Analysis of these explosives on the RA-4100 revealed an inability to detect intact molecular ions, despite the very soft ionization offered by electrospray ionization. Attempts were made to ionize TATP in the negative ion mode, with some success (Figure 115).

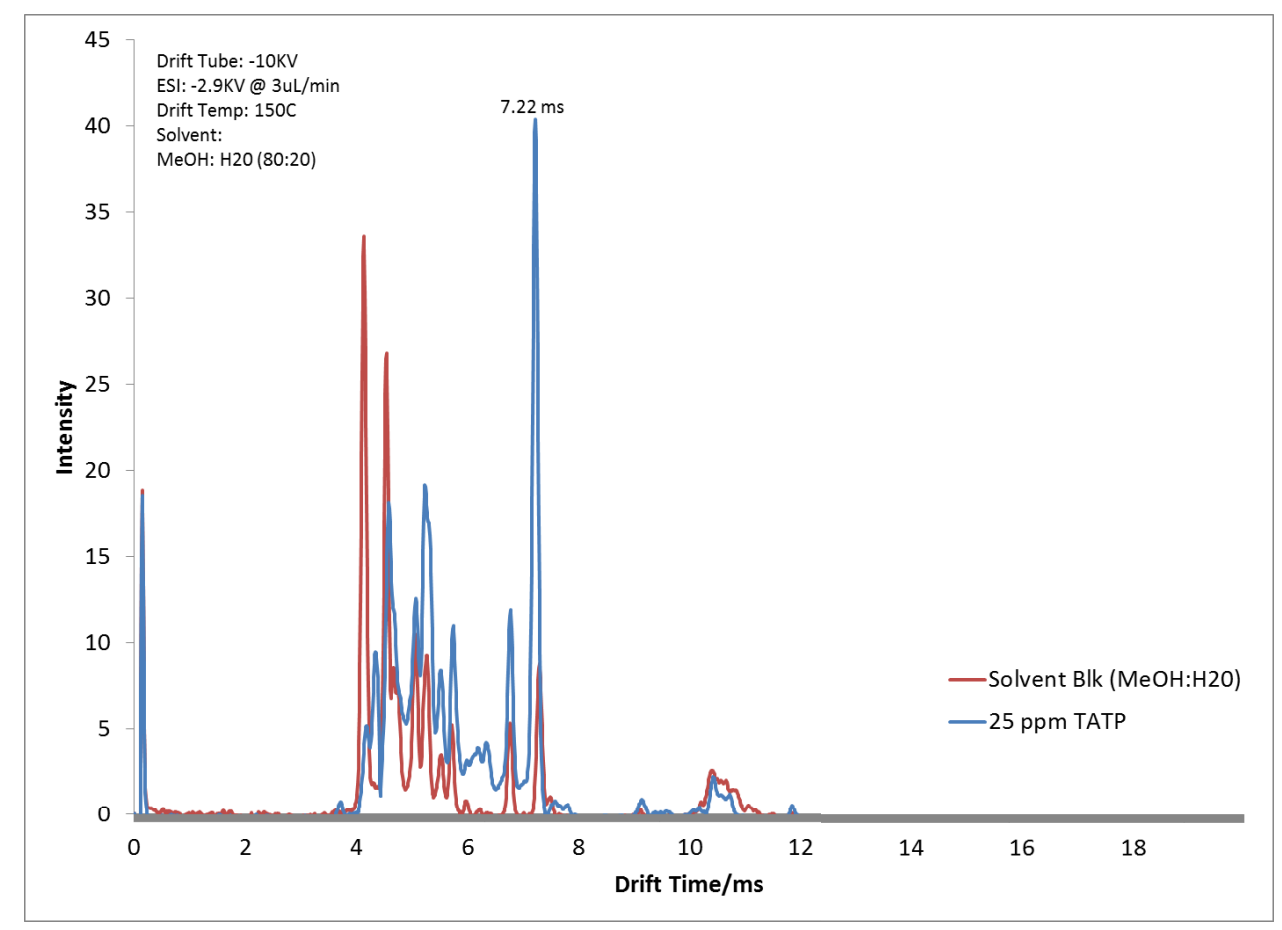

Figure 115: Results from the analysis of TATP in negative ion mode 
The mass spectrum revealed a peak at $\mathrm{m} / \mathrm{z} 168$ that had the same drift time as the observed TATP peak (Figure 116). However the structure of this ion fragment has still not been determined.

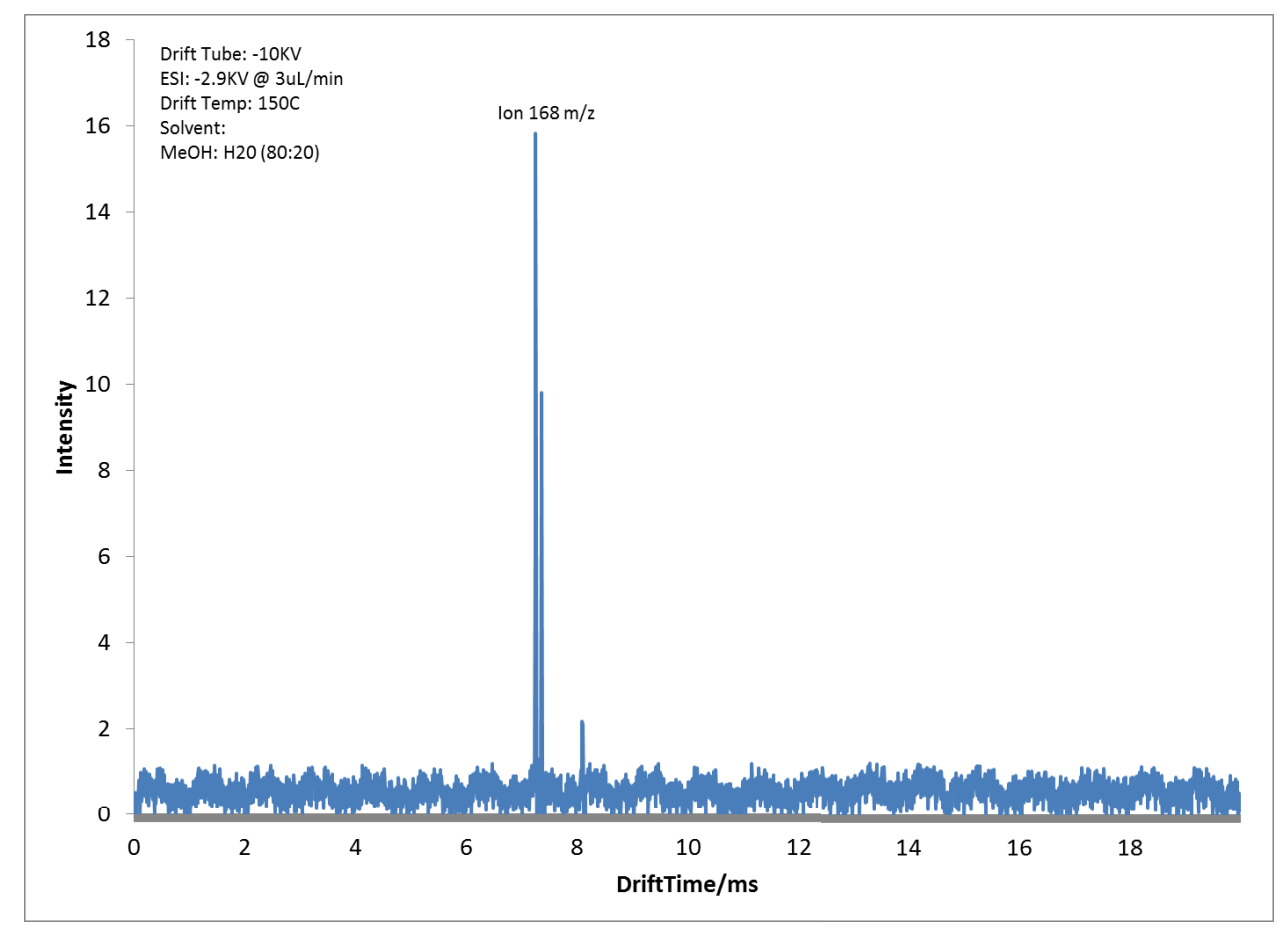

Figure 116: Single Ion Monitoring of TATP ion fragment obtained in the negative ion mode

Though the prospect of obtaining ion responses from peroxide based explosives in the negative mode will simplify and streamline the detection capabilities for IMS devices to detect all explosives whether nitrate or peroxide based, the ion counts obtained in the negative ion mode was very poor. Improved response was obtained for these analytes in the positive ion mode as expected [161;174-176]. Both TATP and HMTD were analyzed in the positive ion mode utilizing optimized solvent systems. Of interest was that both analytes despite having very different molecular masses, produced the same drift times (Figure 117). 


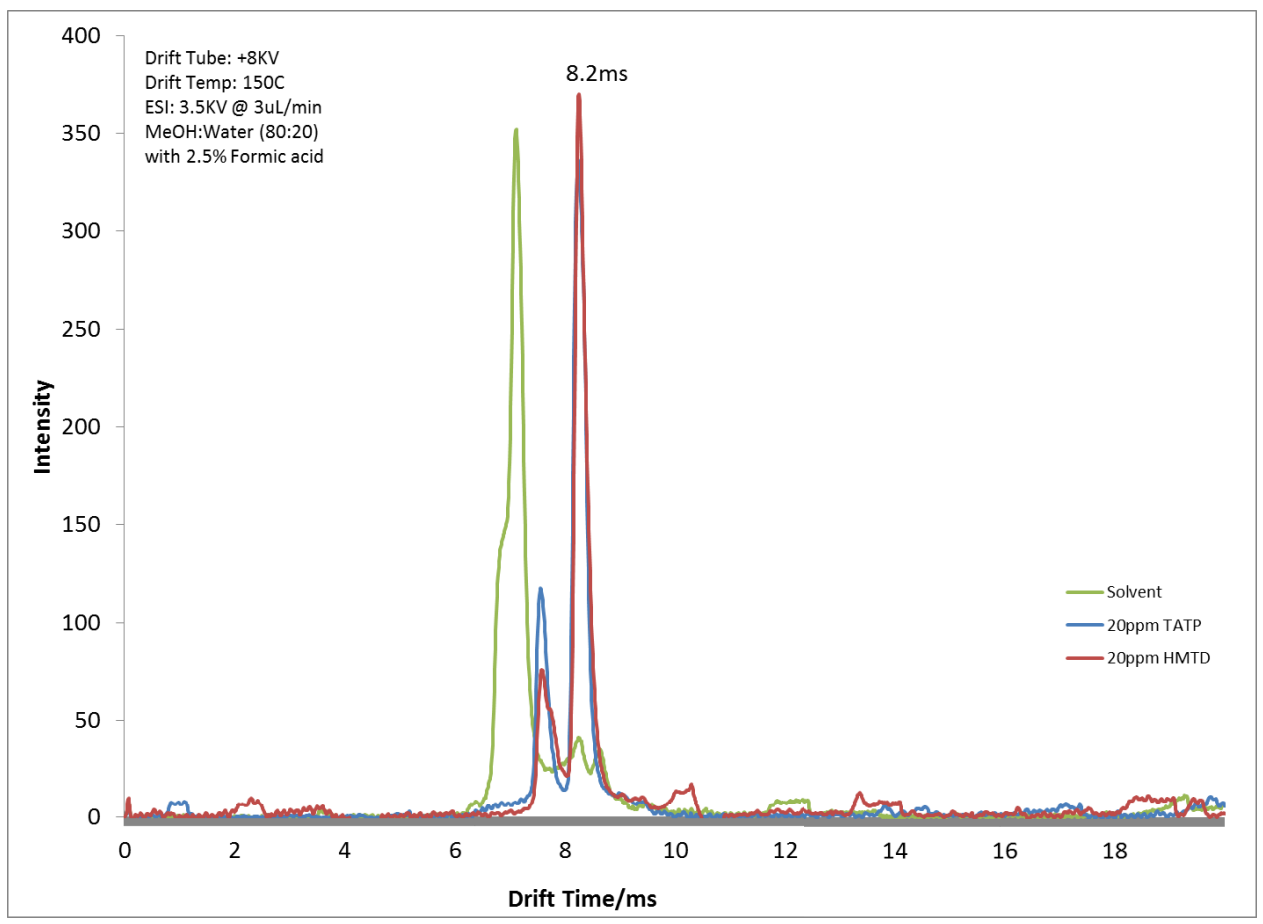

Figure 117: Overlay of 20ppm TATP and 20ppm HMTD detected in positive ion mode

Identical drift times were explained by the obtained mass spectra for both TATP and HMTD. The ESI-IMS Mass Spectrum for both TATP and HMTD were also identical, producing similar fragments and no molecular ions (Figure 118), hence resulting ion identical ions from the analysis of either compound that would in turn have identical drift times. 


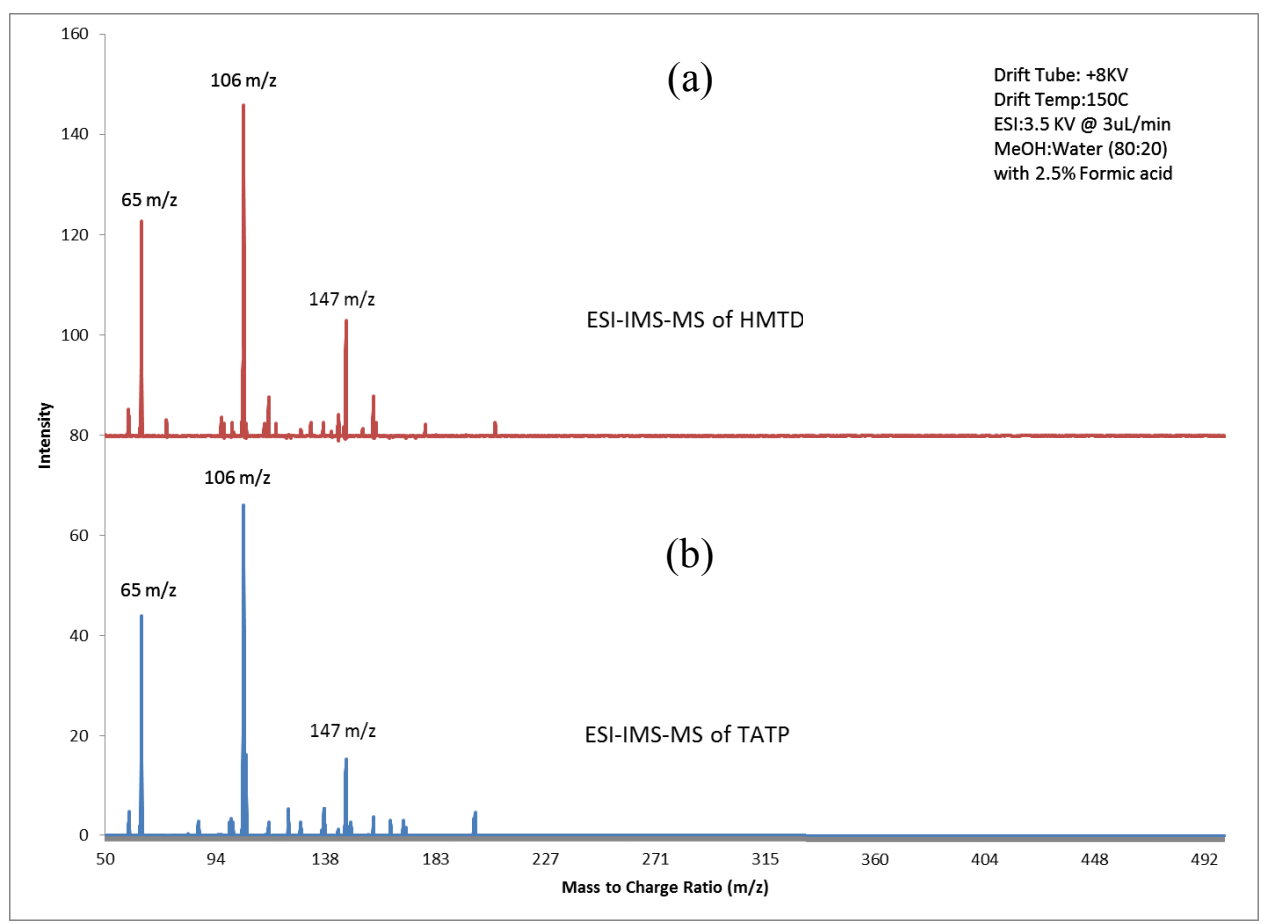

Figure 118: ESI-IMS-MS of (a) HMTD and (b) TATP in positive ion mode using Methanol:Water solvent with $2.5 \%$ formic acid

Fragments were obtained for masses 65, 106 and 147, though fragments at $65 \mathrm{~m} / \mathrm{z}$ are identical to those from the methanol solvent as shown in Figure 62, these ions had a drift time identical to that of ions 106 and 147 which also coincided with the drift time of the peak obtained for both TATP and HMTD of $8.2 \mathrm{~ms}$ (Figure 117). The conclusion then being that the TATP and HMTD molecules also produced a $65 \mathrm{~m} / \mathrm{z}$ ion. Attempts to ionize these peroxide based explosives in order to obtain a molecular ion involved the dissolution of aliquots of TATP and HMTD into a solution comprised of methanol:water (80:20) with $2.5 \%$ formic acid and $10 \mathrm{mMol}$ of Ammonium Nitrate. Based on optimized solvent studies performed, it was believed that the ammonium ion from the dissolved nitrate salt would form an adduct with the analytes in solution, thereby producing a molecular ion that has been previously reported in literature to be the easily obtained molecular structure for TATP $[176 ; 177]$. This solvent system produced identical ion 
mobility spectra to that obtained when utilizing only methanol:water without Ammonium Nitrate, however, the mass spectrum was slightly different than that previously obtained (Figure 119).

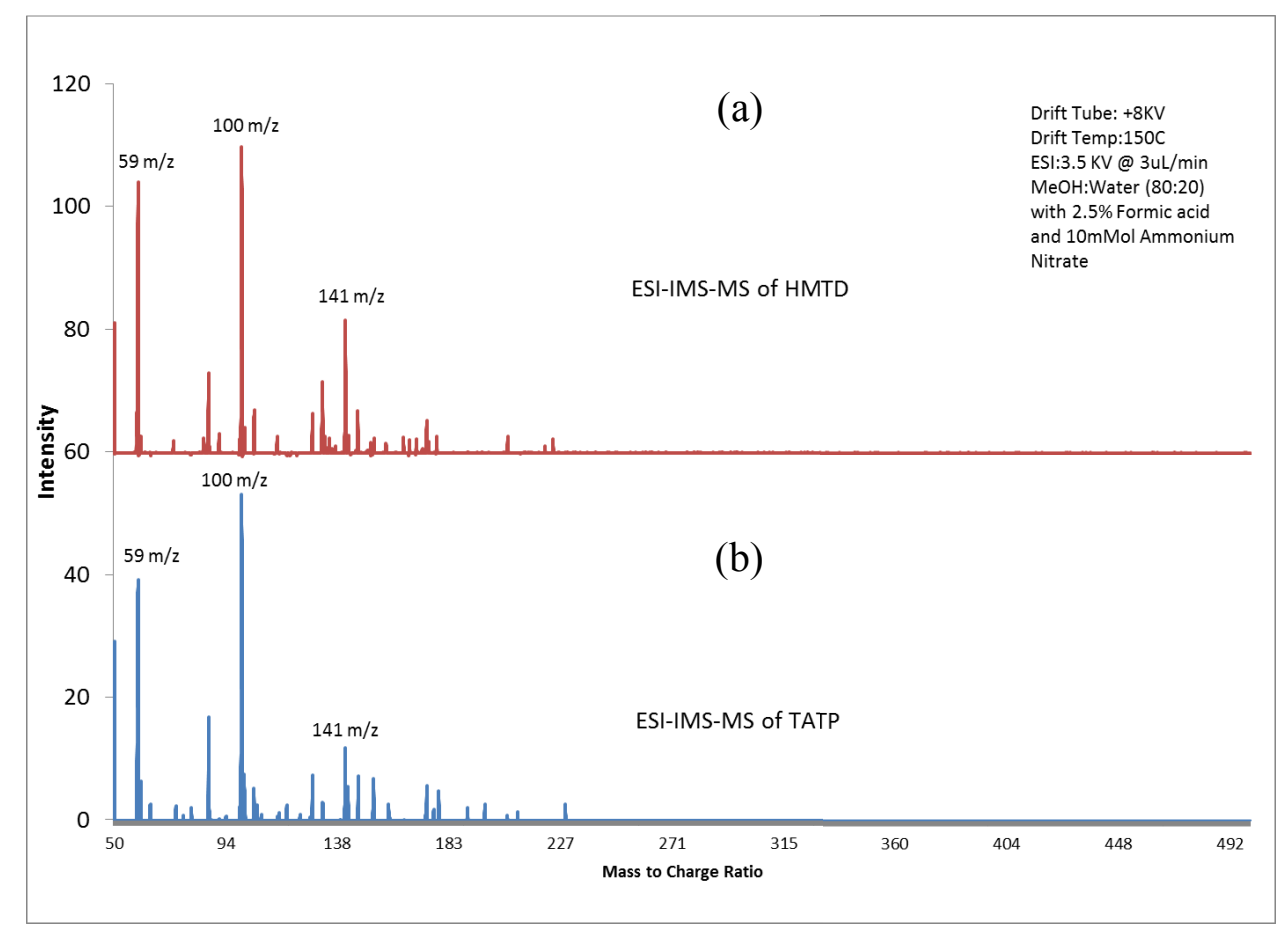

Figure 119: ESI-IMS-MS of (a) HMTD and (b) TATP in positive ion mode using Methanol:Water solvent with $2.5 \%$ formic acid with $10 \mathrm{mMol}$ Ammonium Nitrate

Both HMTD and TATP still produced identical mas spectra, but with ions corresponding to 59,100 and 141 . Of interest was the fact that these ions were exactly 6 amu less than the ions obtained when analyzing these analytes without the ammonium based salt. This has been the source of much confusion as it is now clear that both peroxide based explosives can be analyzed utilizing ESI-IMS-MS and that peroxide based explosives produce identical fragments that are indistinguishable. However, the molecular structure of these ion fragments remains a mystery. Assuming that the ammonium based solvent produced ammonium adducted species $\left(\mathrm{X}+\mathrm{NH}_{4}\right)^{+}$or $(\mathrm{X}+18)^{+}$and that the solvent without 
the ammonium based salt produced a species of $(\mathrm{X}+18+6)^{+}$. The illusive molecular formula of " $\mathrm{X}$ " is yet to be determined. This is coupled to the fact that each observed ion is exactly 41 amu apart in each instance. The exact structure of this 41 amu repeating unit is yet to be determined.

\subsubsection{Nitroglycerin}

Nitroglycerin $(\mathrm{NG})$ or trinitroglycerin (RMM $227.08 \mathrm{~g} / \mathrm{mol}$ ) is one of the oldest explosives still being used. Originally commercialized as dynamite by Sir Alfred Nobel in 1867 by mixing diatomaceous earth with the explosive to improve its handling characteristics, as nitroglycerin is very shock sensitive similar to the peroxide based explosives. As a result, nitroglycerin is rarely used on its own, however, by mixing the explosive with another additive thereby making it less shock sensitive and easier to handle, nitroglycerin has become the single most important ingredient in small arms manufacturing of smokeless powders (Figure 120).

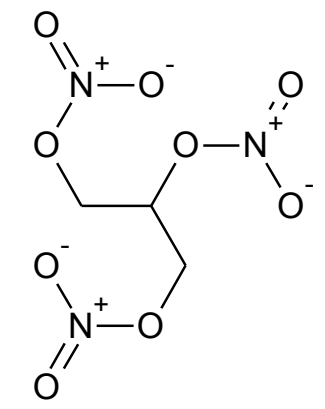

Figure 120: Structure of Nitroglycerin

The structure of nitroglycerin like many other explosives possesses nitro groups that allows for rapid oxygenation of fuels and results in detonation. However, the mixing of nitroglycerin with various additives to control its shock sensitivity has confined its burn rate, causing nitroglycerin containing smokeless powders to only deflagrate. These nitrate 
groups allows nitroglycerin to also be detected in the negative ion mode providing ready detection alongside the other explosive compounds listed in Table 9. Analysis of nitroglycerin by ESI-IMS-MS was achieved and resulted in the detection of an ion mobility peak, when sprayed in the negative mode using a methanol:water mixture. The corresponding mass spectrum was obtained and revealed ions at $62 \mathrm{~m} / \mathrm{z}$ and $289 \mathrm{~m} / \mathrm{z}$. This corresponded to $\left(\mathrm{NO}_{3}\right)^{-}$and $\left(\mathrm{C}_{3} \mathrm{H}_{5} \mathrm{~N}_{3} \mathrm{O}_{9}\right)\left(\mathrm{NO}_{3}\right)^{-}$(Figure 121).

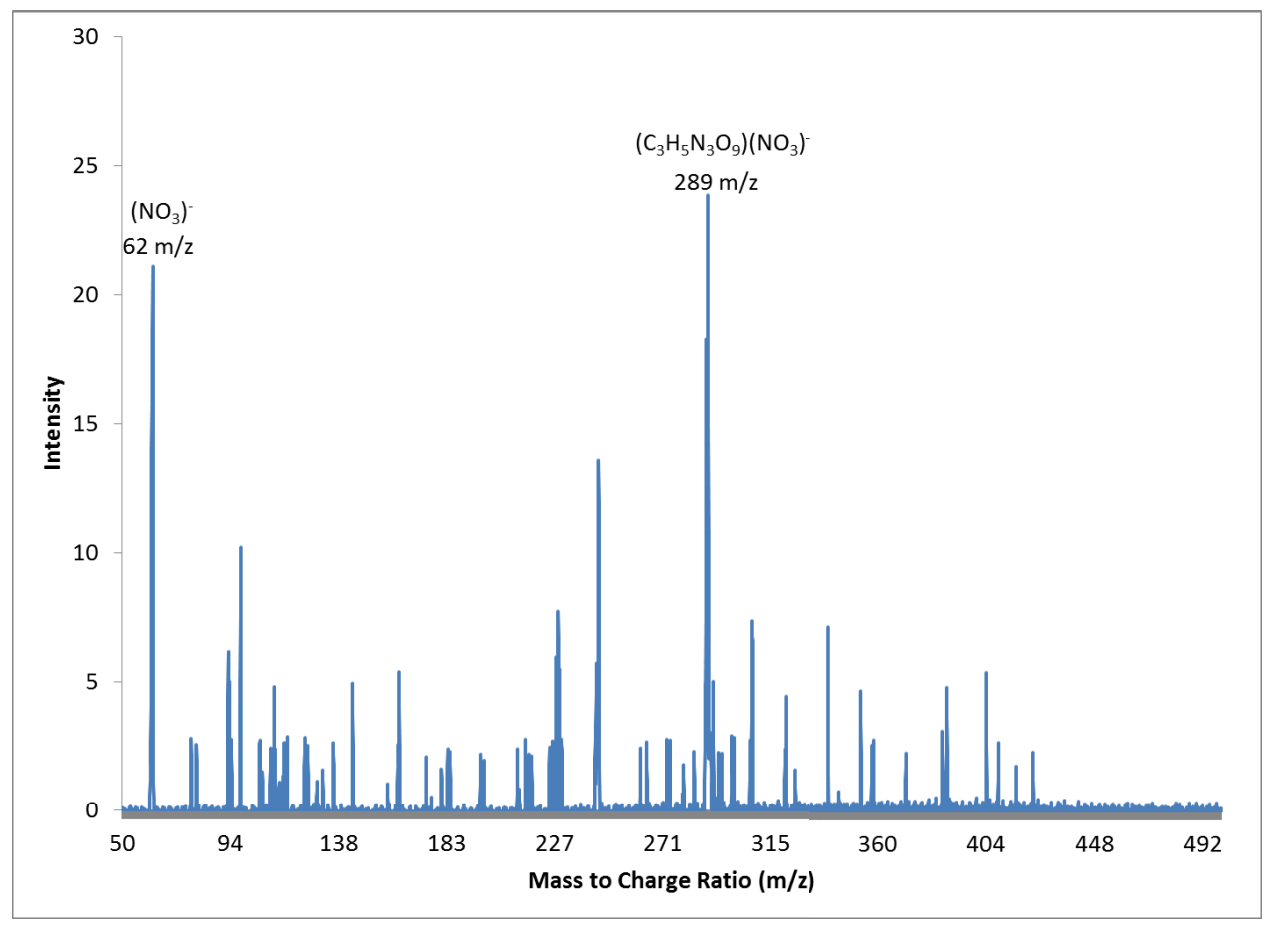

Figure 121: ESI-IMS-Mass Spectrum of Nitroglycerin dissolved in MeOH:Water in the negative ion

\subsubsection{DNT and TNT} mode

The nitro aromatics 2,4 dinitrotoluene (DNT, RMM $182.13 \mathrm{~g} / \mathrm{mol}$ ) and 2,4,6 trinitrotoluene (TNT, RMM $227.13 \mathrm{~g} / \mathrm{mol}$ ) were among the simplest molecules to analyze. These compounds provided relatively simple spectra of a proton abstracted molecular ion. Neither nitro-aromatic compound formed any adducted ionic species, irrespective of the solvent system used. 


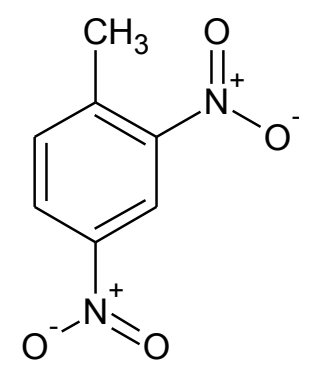

(a)

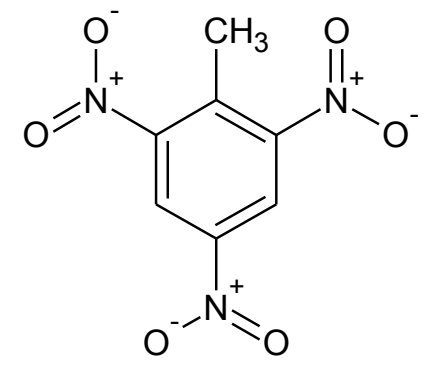

(b)

Figure 122: Structure of (a) 2,4 Dinitrotoluene and (b) 2,4,6 Trinitrotoluene

However, it was discovered that the use of nitrated solutions, such as methanol:water dissolved with ammonium nitrate, completely suppressed the ionization of these nitroaromatics. It is being hypothesized that the cause for this suppression lies within the ability of the nitrated solvent to accept a proton from the nitro-aromatic species. Previous reports into the proton abstraction process that allows negative ionization of analytes at atmospheric pressure, reveals that the charged solvent (BIP) and analyte interact so that a new ion is formed as well as a neutral molecule. If the ESI solvent produces a reactant ion that is unable to accept a proton from the neutral analyte, then ion suppression occurs as observed with nitro-aromatics DNT and TNT when sprayed with nitrate salts such as ammonium nitrate (Figure 123b) [3]. However, if the analyte is able to undergo ion attachment as is the case with nitro-amines in the presence of nitrate salts, then ionization occurs while in this solution. As a result, a mixture of nitro-aromatic and nitro-amines sprayed with a nitrate salt based solution, will only show ionization of the nitro-amines owing to the difference 


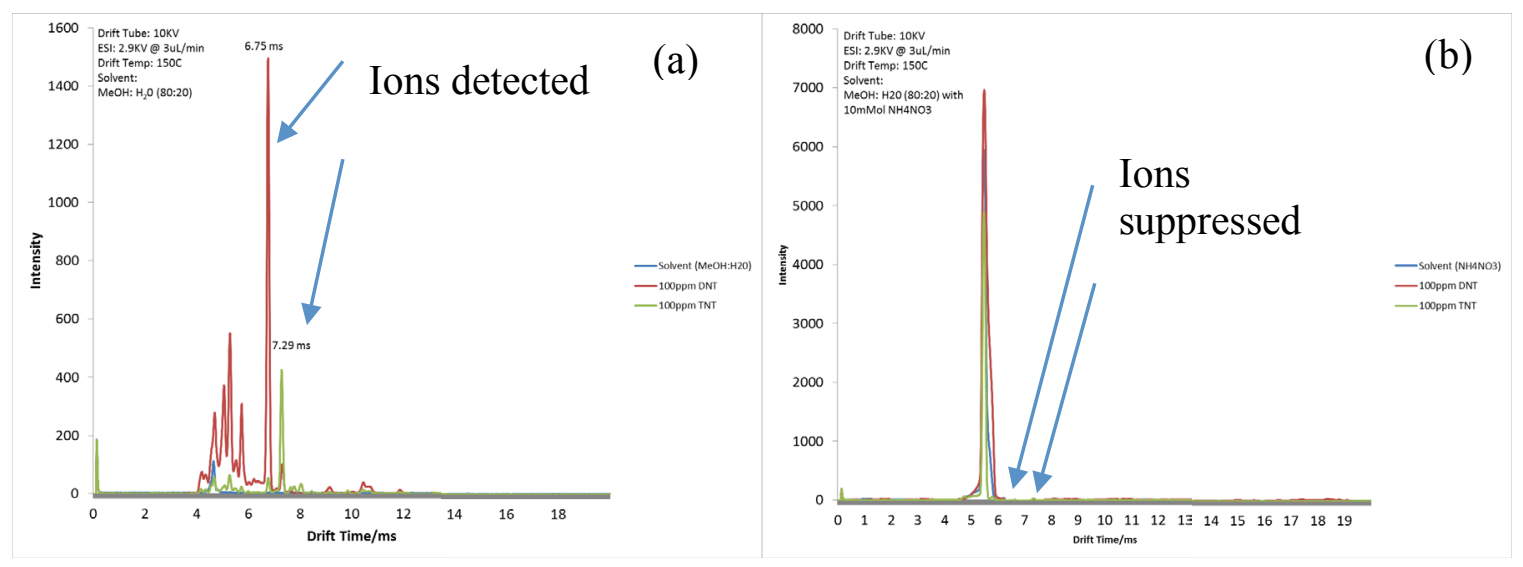

Figure 123: (a) Ionization of Nitro-aromatics in Methanol:Water solution and (b) Suppression of Nitro-aromatics in Nitrate solution

in proton affinity between the nitro-aromatics and the nitrate ions formed during ESI processes [114]. This however is not the case when chloride ions are used. No such suppression was observed with nitro-aromatics and solvents containing chloride ions. During the course of these experiments, no adducted species were formed with the nitroaromatics DNT and TNT with any solution, these species only formed proton abstracted ionic species $[\mathrm{M}-\mathrm{H}]^{-}$.

\subsubsection{Conclusions:}

The utility of the RA4100 (ESI-IMS-MS) for the detection of thermally labile explosives has been demonstrated. These compounds have ranged from highly energetic and unstable nitro-amines such as RDX to non-energetic and thermastable propulsion additives such as 4-nitro-phenylamine. The method of analysis and the ionic species obtained for each analyte of interest has been revealed and the manipulation of the ESI solvent to produce a desired and more responsive analyte. The RA4100 has also been demonstrated to be able to detect analytes present within smokeless powders as well as 
provide sufficient discriminating power for positive mode analysis to classify analyzed smokeless powders by brand.

\subsection{Secondary Electrospray Ionization}

Secondary electrospray ionization is the use of the ionized electrospray solvent to ionize a neutral analyte that is not dissolved in the solvent. This is has been done in other analyses such as Desorption Electrospray Ionization (DESI) [178;179]. The use of secondary electrospray allows the ionization of samples or matrices that are difficult to dissolve in an ESI solvent. It also allows the use of electrospray ionization to ionize completely volatile analytes. It has been reported that using secondary electrospray ionization results in an improvement in ionization efficiency over using conventional electrospray ionization [79]. Armed with this data, experiments were setup that would leverage this improvement for the detection of volatile analytes commonly found within analytes of interest, namely smokeless powders. The advantage of being able to conduct volatile component analysis on forensic samples leads to non-contact sampling of evidence. This typically allows the evidence to be sampled repeatedly without degradation or adulteration from the volatile component test, making this type of testing non-destructive. For smokeless powders in particular, volatile component testing is advantageous for similar reasons, the primary reason being the ability to detect components of a device containing smokeless powders that may be hidden for malicious use as the volatile components constantly evaporate and dissipate away from the device into the surrounding environment, making themselves available for detection. This type of clandestine detection is highly sought after for any type of explosive device. For such 
detection to take place the target analyte must itself be very volatile as a result of it possessing a significantly high vapor pressure. Previous research has revealed a plethora of volatile components that emanate from explosive devices and has also proven that the detection of these volatile components are reliable indicators of the source the actual explosive itself $[29 ; 31 ; 144 ; 147 ; 180-183]$. One component that fits the criteria of having high vapor pressure and being a reliable indicator of the presence of smokeless powder is 2,4 Dinitrotoluene (DNT) [30;147;182]. This analyte is a non-energetic component of many smokeless powder samples and possess a relatively high vapor pressure of $1 \mathrm{mmHg}$ at $102^{\circ} \mathrm{C}$. However, before this analyte can be detected utilizing secondary ionization on the RA4100, the instrument itself had to be modified. The modifications allowed the introduction of a gaseous sample into the drift gas that would then come into contact with the electrospray solvent. Once the neutral gaseous sample interacts with the ionized solvent from the ESI, a transfer of charge takes place allowing the neutral analyte to in turn become ionized.

The modifications were done in-house by swapping the nut and ferrule of the modifier inlet, with a $5 \mathrm{~mm}$ septum and $4.0 \mathrm{~mm}$ internal diameter glass liner $(7.85 \mathrm{~cm}$ length and $6.3 \mathrm{~mm}$ outside diameter.). 


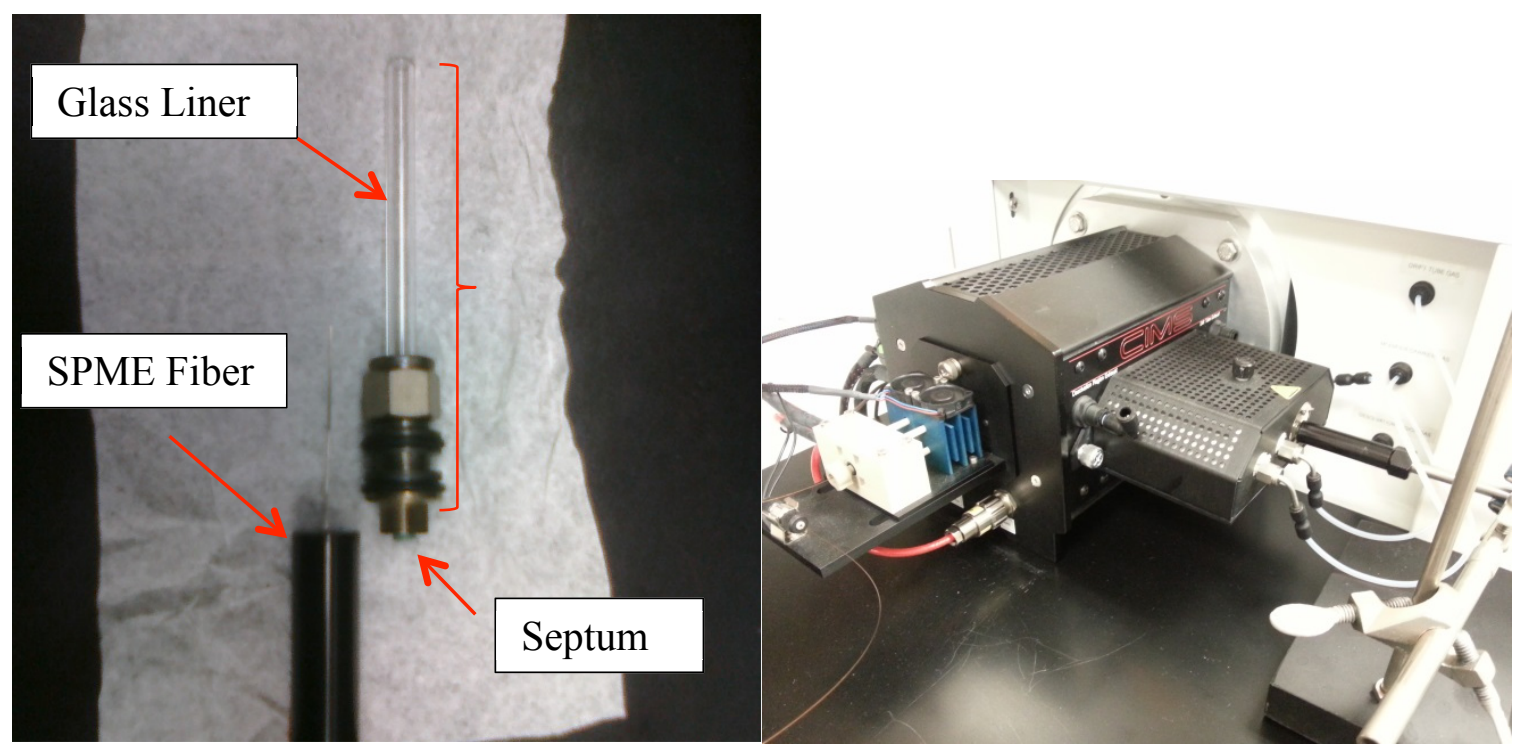

Figure 124: Modifications to allow secondary electrospray ionization on RA4100 with SPME fiber introduction

The first test to verify that the modifications did indeed allow the introduction of the extracted vapor via SPME, was to expose a sample of solid DNT to a SPME fiber and then desorb the fiber in the modifier inlet (Figure 124). For this experiment $94 \mu \mathrm{g}$ of 2,4DNT were deposited in a $15 \mathrm{ml}$ headspace vial and a SPME fiber coated with polydimethylsioxane used to extract the volatile headspace vapor for 30 minutes at room temperature. The ESI solvent was a mixture of Methanol and Water at a ratio of 80:20 $(\mathrm{v} / \mathrm{v})$ and infused at a rate of $3 \mu \mathrm{L}$ per minute. The drift tube was maintained at $150^{\circ} \mathrm{C}$ with Air as the drift gas which was flowed at $1 \mathrm{~L}$ per minute. The modifier temperature was set at $220^{\circ} \mathrm{C}$ to allow sufficient desorption of analyte from the SPME fiber. The modifier gas flow (AIR) was maintained at $1 \mathrm{~L}$ per minute to ensure sufficient mass transport from the modifier into the drift gas and also to give a combined gas flow into the drift tube of $2 \mathrm{~L}$ per minute as this was found to be the optimum drift gas flow rate for this device (Optimization of the Drift Gas Flow and Tube Potential pg. 89). A peak was 
subsequently obtained for the extracted DNT via secondary electrospray ionization on the RA4100 (Figure 125).
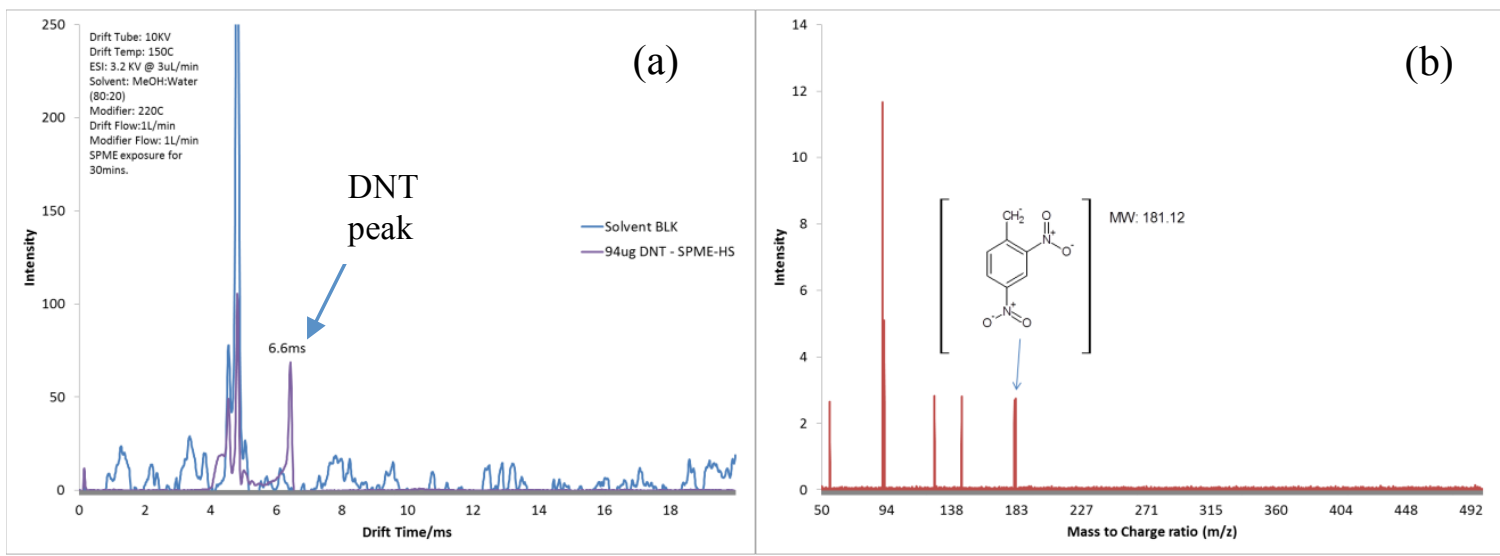

Figure 125: (a) Ion mobility and (b) Mass Spectrum of Secondary ESI of 2,4 DNT collected by SPME Headspace

The main objectives to determine the feasibility of performing Secondary ESI analysis was twofold. The first objective was to enhance the capabilities of the RA4100 by developing and testing a mechanism where volatile compound analysis could be conducted. The second was to verify the claim previously reported in literature that secondary ESI provides an enhancement in sensitivity due to improved ionization efficiency [79]. The first objective was achieved as shown in Figure 125, which shows the detection of 2,4 DNT through SPME headspace analysis. The second objective, which was to provide an enhancement in sensitivity was investigated further through supplemented data via GC-FID to determine the amount of 2,4 DNT that was being extracted by the SPME fiber (Figure 126). 


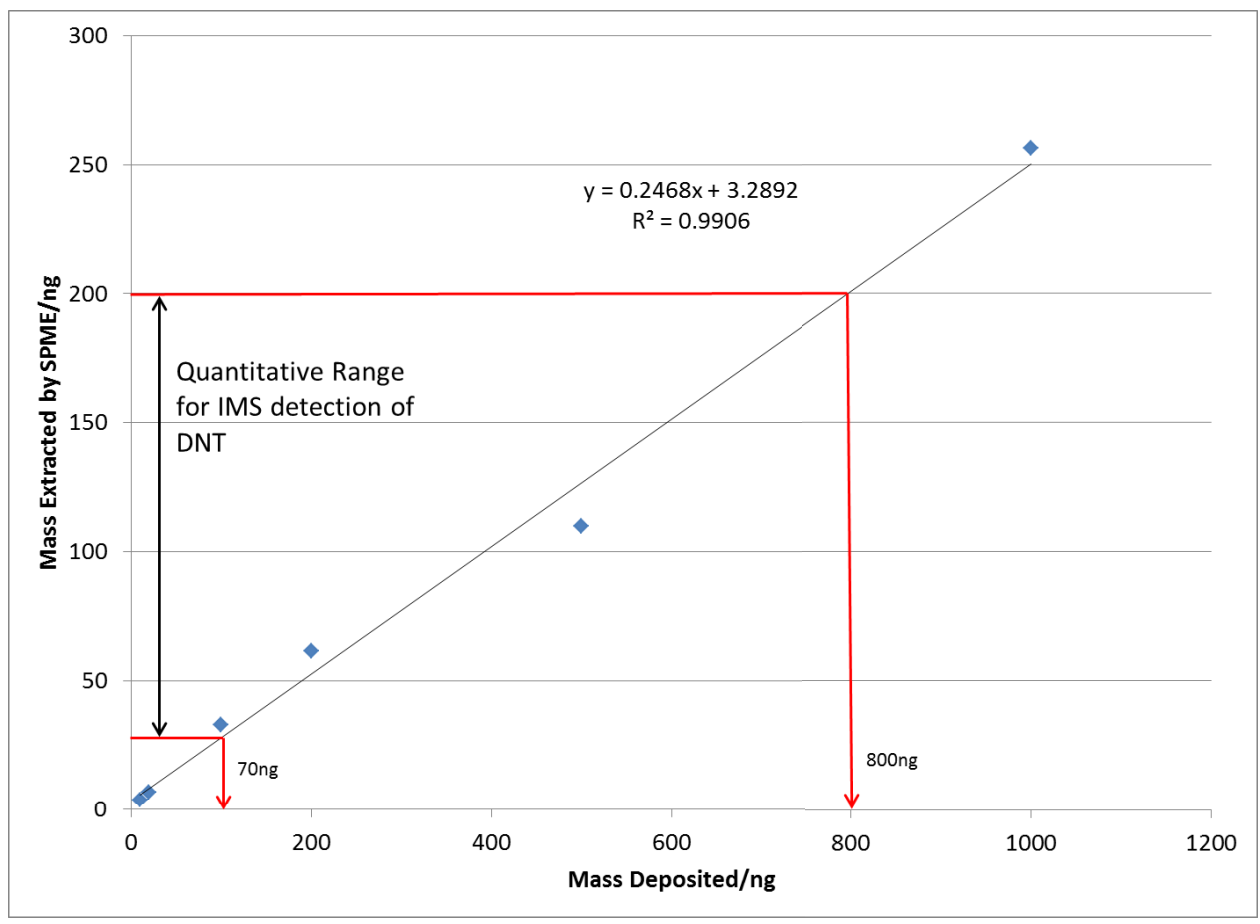

Figure 126: GC-FID data showing amount of DNT extracted by Headspace SPME

The GC-FID data revealed that approximately $24 \%$ of the DNT deposited was being extracted by the SPME fiber. Based on comparisons of the known amounts of DNT deposited in $15 \mathrm{ml}$ vials with a standard response curve of DNT that was directly injected on the GC-FID (Figure 127). 


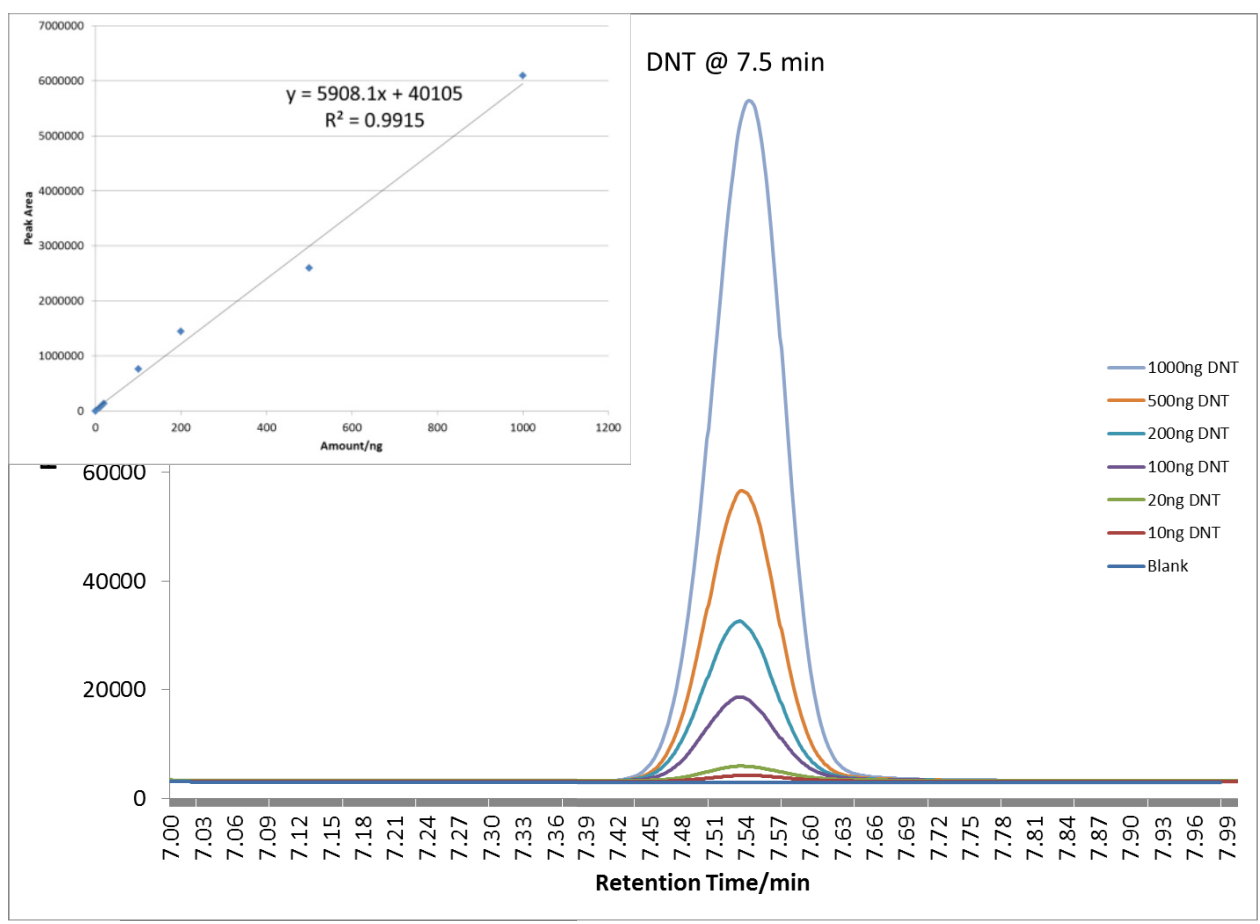

Figure 127: Response of DNT on GC-FID

It was also previously determined that the dynamic range to allow quantitation on the RA4100 for DNT was between $20 \mathrm{ng} / \mu \mathrm{L}$ and $200 \mathrm{ng} / \mu \mathrm{L}$ of DNT as shown in Figure 69. Therefore, the amount of DNT required to be deposited to allow sufficient extraction and produce a suitable response on the RA4100, assuming $24 \%$ extraction efficiency by SPME, would be between 70 and 800ng (Figure 126) which as expected is much less than the 94ug of DNT which was used for the initial experiments. The results of this extraction are shown in Figure 128. A plot of the response obtained following secondary ESI revealed a limit of detection of 110ng of DNT that could be detected and quantified based on the amount of DNT deposited for headspace extraction. This amount of DNT when correlated to graph in Figure 126 equates to approximately 30ng of DNT that was extracted by the SPME fiber. This was an inferior detection limit for DNT dissolved in solution and analyzed by direct ESI as shown in Figure 69. The detection limit for DNT 
dissolved in solution and sprayed by ESI was approximately 10ng versus the 30ng derived by Secondary ESI.

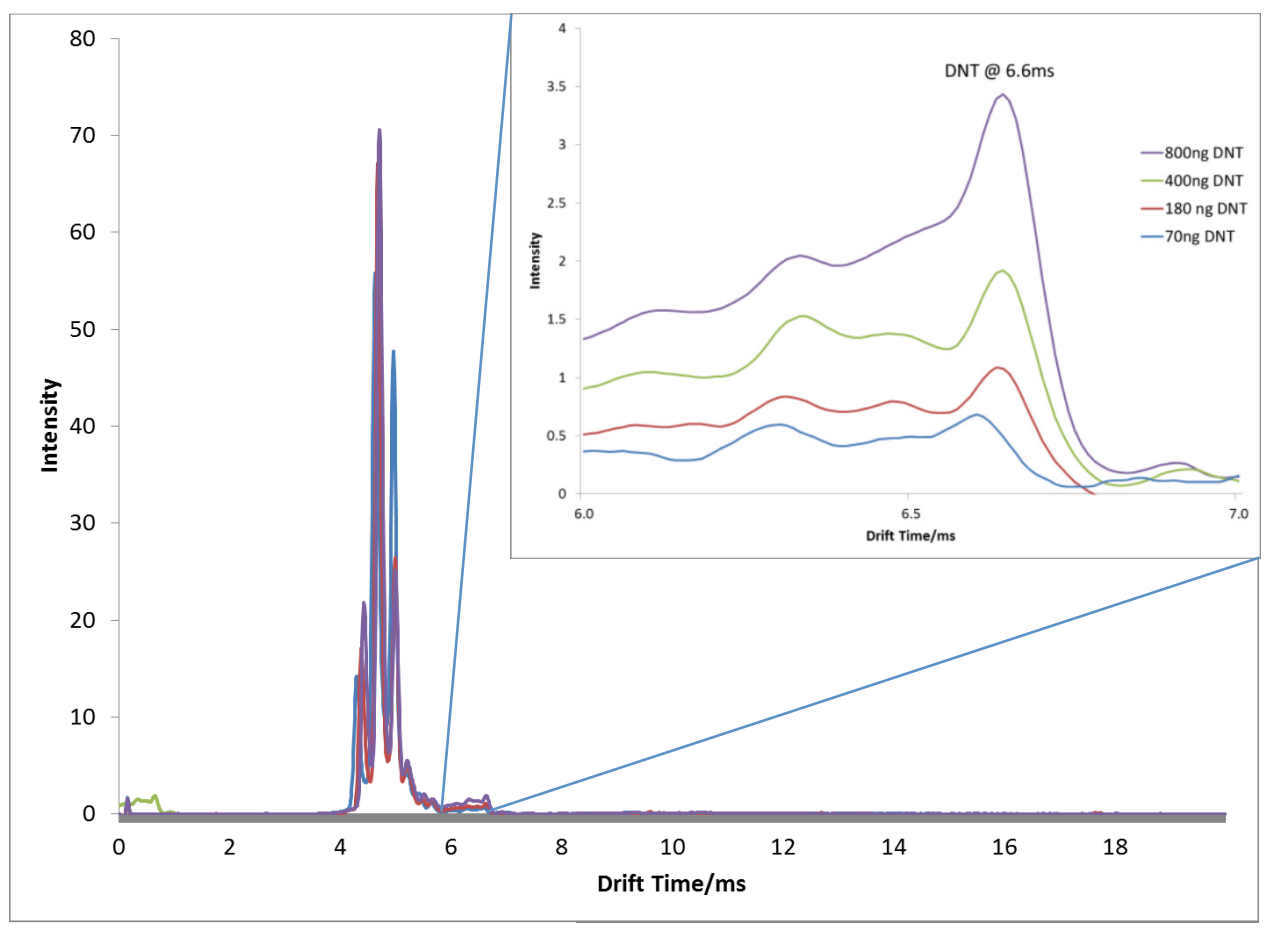

Figure 128: Headspace extraction of DNT by Secondary ESI on RA4100

This corresponds to a poorer detection limit when using secondary electrospray versus that obtained from direct electrospray analysis, contrary to the enhancement reported by Tam et al. [162]. Though, the inferior detection limit for secondary ESI in this present work is believed to be strongly linked to the relatively poor mass transfer from the SPME fiber into the drift gas and then into the drift tube as shown in Figure 129. Despite this, it was demonstrated that the RA4100 is capable of performing volatile compound analysis through secondary electrospray ionization with comparable detection limits to that obtained from direct electrospray analysis. 


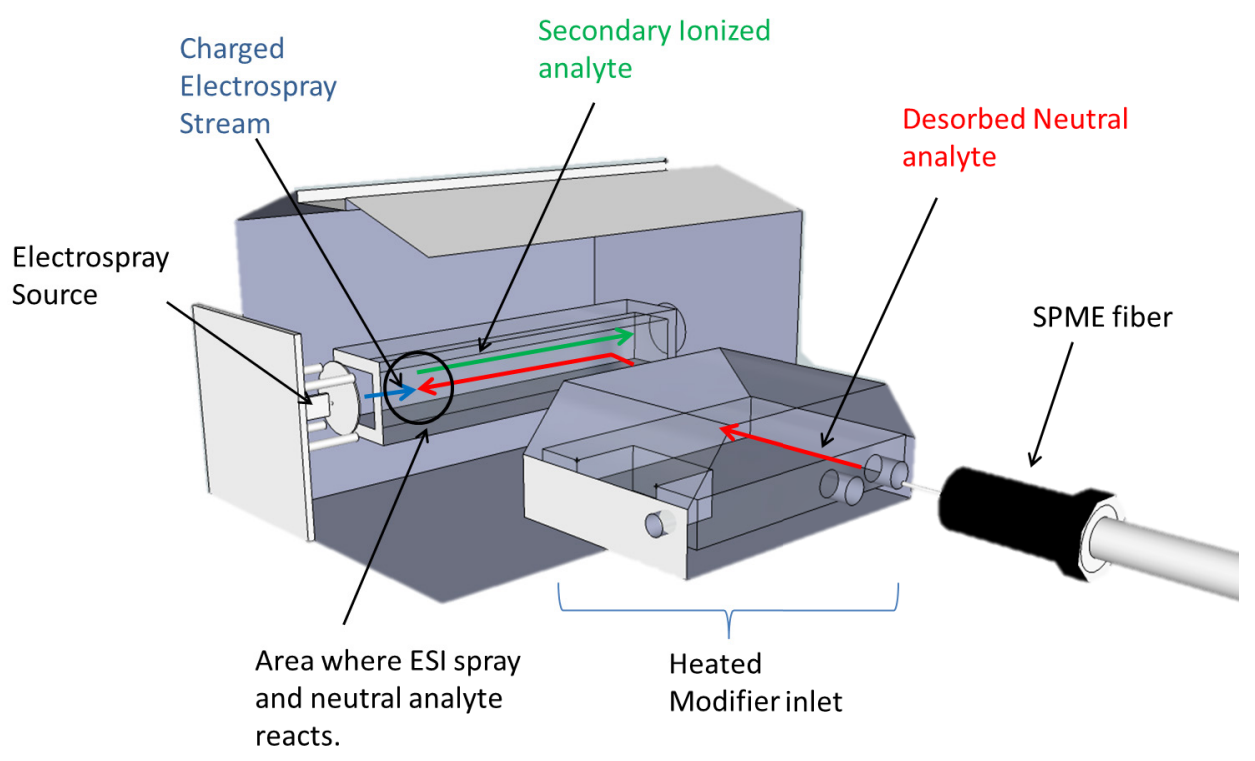

Figure 129: Model of Secondary ESI as it occurs within the RA4100

\subsubsection{Conclusions:}

The novelty of conducting secondary electrospray analysis on the RA4100 is yet another demonstration of the flexibility of this instrument in being able to analyze a wide array of samples. Though poorer detection capabilities were obtained when comparing results of secondary ESI of DNT with that of direct ESI of DNT, this was believed to be mainly attributed to the inefficient mass transfer of desorbed analyte within the RA4100 through the drift tube and ultimately to the ESI source (Figure 129). With improvements in the design of the RA4100 to accommodate introduction of extracted samples for Secondary ESI analysis, a wide variety of volatile vapor samples could also be analyzed with this same technique. 


\section{DISCUSSION}

\subsection{Utilization of Ion Mobility for Chiral Separation}

The ability to perform separations of compounds which are chiral in nature has been demonstrated utilizing both an off the shelf (OTS) Ion Mobility Unit, the Barringer 400B as well as a commercially available Electrospray Ion Mobility Mass Spectrometer (ESIIMS-MS) called the RA4100. The chiral separations conducted and achieved utilizing the OTS IMS, Barringer 400B, verified previously published reports of the effectiveness of using S-2-Butanol as a chiral modifier in separating D and L Methionine. However, significant modifications to the Barringer 400B were required to allow these separations to occur. Modifications included removing the dopant, adding an independent infusion line for the modifier and changes within the system software. Greater improvements in separation were obtained through the use of the Electrospray Ion Mobility Mass Spectrometer (RA4100). The RA4100 was designed with features that allowed introduction of a variety of modifiers which resulted in separations with greater efficiency than that seen with the Barringer 400B. This greater modifier introduction efficiency produced more reliable separations of chiral compounds such as D and L Methionine as well as other compounds of forensic interest namely pseudoephedrines and ephedrines. The more efficient modifier introduction allowed the exploration of other compound such as achiral modifiers namely straight chain alcohols to be used as modifiers. Surprisingly, these achiral modifiers produced similar separations to the chiral modifiers which prompted further investigation as to the exact mechanism of separations occurring. The hyphenation of the mass spectrometer to the IMS allowed for the identification of ions formed within the drift tube during modifier introduction for chiral 
separations. The mass spectrometer revealed new details on the interactions that occur between modifier and chiral analyte. Numerous modifier molecules were observed to interact with each chiral analyte resulting in a molecular ion cluster that possessed a greater effective collision cross sectional area $(\Omega)$ than the molecule previous to modifier introduction. This was found to be contrary to the previously believed Pirkle interaction that dictated interaction of one modifier to one analyte and that the modifier itself had to be chiral in nature. Computational analysis at the B3LYP/6-31g level of theory showed that each chiral molecule though possessing the same cluster of modifier molecules had different cross sectional areas. The differences observed in effective collision cross sectional area for each cluster was as a result of the position of specific functional groups on the chiral analyte itself. For pseudoephedrine and ephedrine, these functional groups were an amine and a hydroxyl group that were on different chiral carbon atoms adjacent to one another on the molecule. The close proximity of these functional groups on adjacent chiral atoms created internal hydrogen bonds that were either more accessible to interact with hydroxyl groups on the modifier molecules or were less accessible depending upon the chirality of the analyte. The position of these functional groups around the chiral carbon atoms resulted in modifier molecules having different spacial arrangements around the chiral analyte inside the IMS drift tube. Compounds with an internal or "bridged" structure arranged modifiers more tightly than compounds without the internal or "independent" structure. This gave bridged compounds a slightly smaller collision cross sectional area than the independent structure and allowed bridged compounds to interact less with the surrounding drift gas and so migrate at a faster rate down the drift tube. The independent structure resulted in slightly larger collision cross 
sectional area, resulting in more interactions with the drift gas and a subsequently slower migration time down the drift tube. These differences were sufficient to cause separations of isomeric chiral compounds within the IMS even when the modifiers being used were not chiral (achiral). The separation mechanism revealed that achiral modifiers were sufficient in causing differences in effective collision cross sectional areas as the chiral analytes themselves were responsible for the arrangement of the cluster ions being formed. Larger modifier molecules resulted in greater separation power and hence the most effective modifier used in the study was n-octanol [13]. This was another significant revelation for chiral separations by ion mobility as previously only modifiers with chiral characteristics were believed to be able to produce such separations.

\subsection{Application of Appropriate Solvent System for Chiral Analysis}

The utilization of an electrospray ionization (ESI) source on the RA4100 offered greater analytical flexibility than that observed with the typical nickel-63 radio nuclei of OTS IMS units. As the ionization of molecules occurs under ambient conditions in the presence of air in IMS, charge competition was a constant inhibitive factor during analysis. However, this was alleviated through the use of selective solvent chemistry, allowing analytes that had differing proton affinities to be ionized simultaneously with the use of ESI. This allowed the RA4100 to simultaneously analyze analytes that would typically be too difficult to ionize utilizing traditional nickel based IMS units. This gave the IMS the ability to analyze mixtures identical to those confiscated and tested in a real forensic setting. This provided simultaneous identification of multiple analytes within the same mixture, and also allowed comparisons to be made of the ratio of chiral analytes 
within the same mixture, providing a mechanism for provenance work to be conducted on these samples. The use of selective solvent chemistries also resulted in improvements in the dynamic response range of analytes, as the solvent promoted increased ionization efficiency resulting in increased ion current generation and hence increased ion cluster detection by the faraday detector. However, it was also noted that the use of very high acid levels within the solvent chemistry for positive ions created wider more diffused peaks, resulting in a reduction in the overall resolving power of the technique. The use of selective solvents therefore must be tapered to balance the need for identifying a complex mixture of analytes without jeopardizing instrument resolution. Formic acid at a concentration of at least $2.5 \%$ was found to show enhanced ionization efficiency in the positive ion mode when compared to solvents with less or no formic acid added. Methyl halides specifically, chloroform at a concentration of $0.1 \%(\mathrm{v} / \mathrm{v})$ was found to promote improved ionization efficiency in the negative ion mode. Other solvent such as ammonium nitrate proved useful in altering the ionic species being obtained for some analytes. For the positive ion mode, such solvent chemistries produced ammonium adducts $\left(\mathrm{M}+\mathrm{NH}_{4}\right)^{+}$versus the typical protonated $(\mathrm{M}+\mathrm{H})^{+}$adducts. In the negative ion mode, this same solvent system produced $\left(\mathrm{M}+\mathrm{NO}_{3}\right)^{-}$adducts versus the typical $(\mathrm{M}-\mathrm{H})^{-}$ ions. However, it was also observed that the ionization of nitro-aromatics was suppressed in the ammonium nitrate solvents. These findings were also published in peer reviewed literature [86]. 


\subsection{Development of IMS methods for the detection of Thermally Labile Explosive Compounds.}

Analysis of thermally labile compounds commonly found in explosives has been a special niche area for IMS since its inception. However an understanding of the ions generated during these analyses had not previously been fully investigated. The hyphenated ion mobility mass spectrometer was able to elucidate the ionic species of these very fragile compounds, revealing that some ions form only as adducts, such as the nitro-amines, while other ions form proton abstracted species such as the nitro-aromatics. Still other compounds formed such fragmented species that a molecular formula has yet to be determined such as the peroxide explosives. Most of these compounds were analyzed in the negative ion mode and selective solvent chemistries again played a critical role in determining the ionization efficiency as well as the type of ionic species that would be formed and subsequently detected. Interesting observations such as the complete ionization suppression of nitro-aromatics in the presence of nitrate based solutions was observed. Other observations included the generation of chloride adducts from solvents spiked with methyl halides such as chloroform during electrospray ionization in the negative ion mode. These experiments with solvent chemistries formed the basis of tests that were subsequently used for the analysis of real world low explosives (smokeless powders) that were extracted and prepared in the appropriate solvent for ESI-IMS-MS analysis.

In all thirty six smokeless powder samples from a variety of brands were extracted and subject to analysis by ESI-IMS-MS analysis on the RA4100 utilizing both positive and negative ionization. The ability to simultaneously ionize analytes from within a sample 
(multiplex) allowed for statistical discriminant analysis to be done. It was revealed that the smokeless powder samples were not simply analyzed and identified as smokeless powders, but were able to be classified by brand. ESI-IMS-MS proved just as viable as classical analysis utilizing GC-MS. In addition, the analysis time for these samples by ESI-IMS-MS was reduced to a fraction of their current values when compared to GC-MS or LC-MS analysis. ESI-IMS-MS analysis was performed in 20 milliseconds from the most rapid currently reported analysis time of 8 minutes utilizing LC-MS [159], with comparable results.

\subsection{Secondary Electrospray Ionization}

The true versatility of the RA4100 was demonstrated by the ability to analyze volatile organic compounds extracted with a solid phase microextraction (SPME) fiber. With slight changes to the modifier inlet of the RA4100, a SPME fiber was able to desorb extracted volatile organic compounds directly into the drift gas of the instrument allowing secondary ionization to occur of the extracted analyte. For these tests, the volatile compound Dinitrotoluene was tested and found to be extracted, ionized and detected by both the IMS and the MS. The only downfall being the poorer sensitivity obtained from the secondary ionization process, most likely from the less efficient VOC introduction

mechanism that transports neutral extracted VOCs from the SPME fiber through the drift tube and ultimately to the electrospray ionization source where secondary ionization occurs. Improvements in the mass transport of these extracted neutrals into the drift tube will ultimately allow for greater sensitivity with this application of the IMS. 


\subsection{Overall Conclusions:}

In all, a complete suite of experiments were carried out utilizing Ion Mobility Mass Spectrometry. Higher resolving powers were obtained, approximately 80 versus 35, when comparing the ESI-IMS-MS and the Barringer 400B Nickel-63 based ion mobility units respectively. The use of an electrospray ionization source offered the ability to use selective solvent chemistries to aid in the ionization of analytes thereby allowing the ionization and subsequent analysis of a mixture of analytes simultaneously (multiplexing). The manipulation of solvent chemistry also showed improvements in the detection capabilities of the instrument in the positive mode when utilizing formic acid and in the negative mode when utilizing chloroform. The modifier on the RA4100 was essential in allowing superior chiral separation capabilities versus that which could be obtained from utilizing the OTS Barringer 400B. Resolution was obtained for chiral compounds Pseudoephedrine and Ephedrine utilizing achiral modifiers. The mass spectrometer on the RA4100 revealed the mechanism of interaction of the modifier during chiral separations, revealing the rational for the use of less expensive achiral modifiers as well as the type of compounds that may be separated. Detection and characterization of a wide variety of thermally labile compounds were also achieved, again revealing the importance of solvent chemistry makeup on the type of ionic species obtained. Application of the techniques developed in manipulating ESI solvent chemistries were used to analyze a battery of low explosive samples resulting in the classification of samples by brand. The possibility of conducting solid phase microextraction analysis (SPME) was also done. The secondary ionization experiments with SPME, though successful, proved to be of little value as the sensitivity obtained by 
conducting SPME analysis was significantly lower than that obtained through direct ESI. This poorer sensitivity through secondary ESI was believed to be as a result of inefficient mass transport of the neutral analyte from the SPME fiber in the modifier inlet with the current instrument configuration.

Finally, techniques have been developed for the analysis of a wide variety of analytes of forensic interest utilizing ESI-IMS-MS. The use of ESI-IMS-MS proved to be a multifaceted technique that would be well suited within a forensic setting to provide high speed and low cost analysis of explosives, narcotics, volatile, non-volatile and even chiral compounds. The RA4100 is able to rival results obtained from current LC and GC-MS techniques while providing these at a fraction of the time, milliseconds versus minutes. The use of ESI and the coupling of a mass spectrometer have made the IMS a true analytical technique that should ultimately find its way into more laboratories of both academics and forensic practitioners alike. 


\section{LIST OF REFERENCES}

1. Eiceman GA and Karpas Z (2005) Ion Mobility Spectrometry. CRC Press.

2. G.Reid Asbury and Herbet H.Hill (1999) Journal of Microcolumn Separations 12:172-178.

3. Ewing RG, Atkinson DA, Eiceman GA, and Ewing J (2001) Talanta 54:515-529.

4. Spangler GE and Lawless PA (1978) Anal. Chem. 50:884-891.

5. O'Donnel RM, Sun X, and de B.Harrington P (2008) Trends in Analytical Chemistry 27:44.

6. Guharay SK, Dwivedi P, and Hill HH (2008) Ieee Transactions on Plasma Science 36:1458-1470.

7. Eiceman GA (2002) Trends in Analytical Chemistry 21:259-275.

8. Cottingham K (2003) Anal. Chem. 75:435A-439A.

9. Ward TJ and Ward KD (2011) Anal. Chem. 84:626-635.

10. Smith SW (2009) Toxicol. Sci. 110:4-30.

11. Noggle ET, Deruiter J, and Clark CR (1986) Anal. Chem. 58:1643-1648.

12. Dwivedi P, Wu C, Matz LM, Clowers BH, Siems WF, and Hill HH (2006) Anal. Chem. 78:8200-8206.

13. Holness H, Jamal A, Mebel A, and Almirall J (2012) Analytical and Bioanalytical Chemistry 404:2407-2416.

14. Mie A, Jornten-Karlsson M, Axelsson BO, Ray A, and Reimann CT (2007) Anal. Chem. 79:2850-2858.

15. Mie A, Ray A, Axelsson BO, Karlsson M, and Reimann CT (2008) Anal. Chem. $80: 4133-4140$.

16. Collin OL, Niegel C, DeRhodes KE, Mccord BR, and Jackson GP (2006) Journal of Forensic Sciences 51:815-818.

17. Douse JMF (1981) Journal of Chromatography 208:83-88.

18. Goodrich JN (2002) Tourism Management 23:573-580.

19. United Nations Office on Drugs and Crime. World Drug Report. 6-24-2009. 
20. United Nations Office on Drugs and Crime. World Drug Report 2011. 2011.

21. UNODC. World Drug Report 2012. Sales No. E.11XI.10. 2011. New York, United Nations Publication.

22. Chinaka S, Iio R, Takayama N, Kodama S, and Hayakawa K (2006) J. Health Sci. $52: 649-654$

23. Cladrowarunge S, Hirz R, Kenndler E, and Rizzi A (1995) J. Chromatogr. A. $710: 339-345$.

24. Strano-Rossi S, Botr $\Phi$ F, Bermejo AM, and Tabernero MJs (2009) Forensic Sci int 193:95-100.

25. Iwata $\mathrm{YT}$, Inoue $\mathrm{H}$, Kuwayama K, Kanamori T, Tsujikawa K, Miyaguchi H, and Kishi T (2006) Forensic Sci int 161:92-96.

26. E. Bernier. Explosive signatures: Pre \& Post Blast. 2008. University of Rhode Island.

27. Jehuda Yinon and Jeh. Detection of Hidden Explosives: An Overview. American Laboratory 38[12], 18-23. 2006.

28. Xu XM, van de Craats AM, Kok EM, and de Bruyn PCAM (2004) Journal of Forensic Sciences 49:1230-1236.

29. Jehuda Yinon (2002) Trends in Analytical Chemistry 21:292.

30. Joshi M, Rigsby K, and Almirall JR (2011) Forensic Sci int 208:29-36.

31. Lai H, Leung A, Magee M, and Almirall JR (2010) Analytical and Bioanalytical Chemistry 396:2997-3007.

32. Yinon J, McClellan JE, and Yost RA (1997) Rapid Commun. Mass Sp. 11:19611970.

33. Yinon J, McClellan JE, and Yost RA (2000) Abstracts of Papers of the American Chemical Society 220:146-ANYL.

34. Yinon J, Yost RA, and Bulusu S (1994) J. Chromatogr. A. 688:231-242.

35. WILLIAMS AF, MURRAY WJ, and GIBB BH (1966) Nature 210:816-817.

36. Makinen M, Nousiainen M, and Sillanpaa M (2011) Mass Spectrom. Rev. 30:940-973.

37. Reid Asbury G, Klasmeier Jr, and Hill J (2000) Talanta 50:1291-1298. 
38. Pumera M (2008) Electrophoresis 29:269-273.

39. D.S.Moore (2004) American Institute of Physics 75.

40. Cotte-Rodriguez I, Hernandez-Soto H, Chen H, and Cooks RG (2008) Anal. Chem. 80:1512-1519.

41. Flanigan PM, Brady JJ, Judge EJ, and Levis RJ (2011) Anal. Chem. 83:71157122 .

42. Thurman EM, Ferrer I, and Barcelo D (2001) Anal. Chem. 73:5441-5449.

43. Gunzer F, Ulrich A, and Baether W (2010) International Journal of Ion Mobility Spectrometry 13:9-16.

44. Harvey SR, MacPhee CE, and Barran PE (2011) Methods 54:454-461.

45. Cody RB (2002) Electrospray Ionization Mass Spectrometry. In: Applied Electrospray Mass Spectrometry. Marcel Dekker, New York.

46. Cech NB and Enke CG (2001) Mass Spectrom. Rev. 20:362-387.

47. Kebarle P and Verkerk UH (2009) Mass Spectrom. Rev. 28:898-917.

48. Blades AT, Ikonomou MG, and Kebarle P (1991) Anal. Chem. 63:2109-2114.

49. Fenn JB, Mann M, Meng CK, Wong SF, and Whitehouse CM (1990) Mass Spectrom. Rev. 9:37-70.

50. Budnik BA, Tsybin YO, Hakansson P, and Zubarev RA (2002) J Mass Spectrom 37:1141-1144.

51. Dole M, Mack LL, Hines RL, Mobley RC, Fergusan LD, and Alice MB (1968) Journal of Chemical Physics 49:2240-2249.

52. Taylor G (1964) Proceedings of the Royal Society of London. Series A. Mathematical and Physical Sciences 280:383-397.

53. Rayleigh JWS (1896) The Theory of Sound. MacMillan and Company, New York.

54. Beckey HD (1971) Field ionization mass spectrometry. Pergamon Press, Oxford, New York.

55. Dempster AJ (1918) Physics review 11:316-325.

56. Aston F.W. Experiments on a New Cathode Dark Space in Helium and Hydrogen. The Royal Society of London 80[535], 45-49. 12-7-1907. 
57. Gordon Squires (1998) Dalton transactions3893-3899.

58. M. Geisow. New developments in biochemical mass spectrometry. Biologicals 21[2], 125-129. 1993.

59. Oberacher H, Niederstatter H, Casetta B, and Parson W (2005) Anal. Chem. 77:4999-5008.

60. Oberacher H (2008) Analytical and Bioanalytical Chemistry 391:135-149.

61. McDaniel EW, Martin DW, and Barnes WS (1962) Review of Scientific Instruments 33:2-7.

62. Zhu M, Bendiak B, Clowers B, and Hill H (2009) Analytical and Bioanalytical Chemistry 394:1853-1867.

63. Bornschein RE, Hyung SJ, and Ruotolo BT (2011) Journal of the American Society for Mass Spectrometry 22:1690-1698.

64. J. Kapron and D. A. Barnett. Selectivity Improvement for Drug Urinalysis using FAIMS and H-SRM on the Finnigan TSQ Quantum Ultra. Application Note 362. 2012. Thermo Electron Corporation.

65. D. E. Hunka and D. E. Austin. Ion Mobility Spectrometry/Mass Spectrometry (IMS-MS). 2006. Sandia National Laboratories. SAND2005-6908.

66. U.S Department of Justice Drug Enforcement Administration. Scientific Working Group for the Analysis of Seized Drugs (SWGDRUG) Recommendations. 4th Edition. 10-1-2008. SWGDRUG Recommendations.

67. Borsdorf H and Eiceman GA (2006) Applied Spectroscopy Reviews 41:323-375.

68. Ficarro SB, Zhang Y, Lu Y, Moghimi AR, Askenazi M, Hyatt E, Smith ED, Boyer L, Schlaeger TM, Luckey CJ, and Marto JA (2009) Anal. Chem. 81:34403447.

69. Arce L, Menendez M, Garrido-Delgado R, and Valcarcel M (2008) Trac-Trends in Analytical Chemistry 27:139-150.

70. Jafari MT (2009) Talanta 77:1632-1639.

71. J. Gieniec, H. L. Cox, D. Teer, and M. Dole. Application of the Ion-Drift Spectrometer to Macromass Spectrometry. Twentieh Annual Conference on mass Spectrometry and Applied Topics. Twentieh Annual Conference on mass Spectrometry and Applied Topics , 276. 6-4-1972. 6-4-1972. 
72. Shumate CB and Hill HH (1989) Anal. Chem. 61:601-606.

73. Wittmer D, Luckenbill BK, Hill HH, and Chen YH (1994) Anal. Chem. 66:23482355.

74. Wu C, Siems WF, Asbury GR, and Hill HH (1998) Anal. Chem. 70:4929-4938.

75. Jafari MT, Rezaei B, and Javaheri M (2011) Food Chemistry 126:1964-1970.

76. Covey TR and Pinto D (2002) Nanospray Electrospray Ionization Development. In: Applied Electrospray Mass Spectrometry. Marcel Dekker, New York.

77. Tang XT, Bruce JE, and Hill HH (2006) Anal. Chem. 78:7751-7760.

78. Griffiths WJ, Jonsson AP, Liu S, Rai DK, and Wang Y (2001) Journal of Biochemistry 355:545-561.

79. Wu C, Siems WF, and Hill HH (2000) Anal. Chem. 72:396-403.

80. Wu ZR, Gao WQ, Phelps MA, Wu D, Miller DD, and Dalton JT (2004) Anal. Chem. 76:839-847.

81. Cole RB and Harrata AK (1993) Journal of the American Society for Mass Spectrometry 4:546-556.

82. Mathis JA and Mccord BR (2005) Forensic Sci int 154:159-166.

83. Matz LM and Hill HH (2001) Anal. Chem. 74:420-427.

84. Straub RF and Voyksner RD (1993) Journal of the American Society for Mass Spectrometry 4:578-587.

85. B. Bazzi. Ionization competitors extend the linear range of Electrospray ionization mass spectrometry. Thesis 2009. The University of Texas at Arlington.

86. Holness H and Almirall J (2013) Int. J. Ion Mobil. Spec. 16:237-246.

87. Eiceman GA, Nazarov EG, and Stone JA (2003) Analytica Chimica Acta 493:185-194.

88. Fernandez-Maestre R, Harden CS, Ewing R, Crawford CL, and Herbert H Hill Jr (2010) Analyst 135:1433-1442.

89. Vautz W, B+,deker B, Baumbach J+, Bader S, Westhoff M, and Perl T (2009) International Journal for Ion Mobility Spectrometry 12:47-57. 
90. William A.Munro, C.L.Paul Thomas, and Marian L Langford (1998) Analytica Chimica Acta 375:49-63.

91. Colin S.Creaser, John R.Griffiths, Claire J.Bramwell, Sadaf Noreen, Carol A.Hill, and C.L.Paul Thomas (2004) Analyst 129:984-994.

92. Buryakov IA (2002) Technical Physics 47:1453-1457.

93. Krylov EV and Nazarov EG (2009) International Journal of Mass Spectrometry 285:149-156.

94. Borsdorf H, Nazarov EG, and Miller RA (2006) Analytica Chimica Acta 575:7688.

95. Eiceman GA and Feng Y (2009) J. Chromatogr. A. 1216:985-993.

96. Eiceman GA, Krylov EV, Nazarov EG, and Miller RA (2004) Anal. Chem. 76:4937-4944.

97. Krylov EV, Nazarov EG, and Miller RA (2007) International Journal of Mass Spectrometry 266:76-85.

98. Krylov EV, Coy SL, and Nazarov EG (2009) International Journal of Mass Spectrometry 279:119-125.

99. Manard MJ, Trainham R, Weeks S, Coy SL, Krylov EV, and Nazarov EG (2010) International Journal of Mass Spectrometry 295:138-144.

100. Nazarov EG, Miller RA, Eiceman GA, and Stone JA (2006) Anal. Chem. 78:4553-4563.

101. Nazarov EG, Coy SL, Krylov EV, Miller RA, and Eiceman GA (2006) Anal. Chem. 78:7697-7706.

102. Smiths Detection. History of Barringer. World Wide Web . 2013. 1-3-2013.

103. Kanu AB, Dwivedi P, Tam M, Matz L, and Hill HH (2008) J Mass Spectrom 43:1-22.

104. Vautz W, Michels A, and Franzke J (2008) Analytical and Bioanalytical Chemistry 391:2609-2615.

105. Kim SH, Betty KR, and Karasek FW (2002) Anal. Chem. 50:2006-2012.

106. D. Pearson. Safran to buy rest of GE's Morpho Detection stake. World Wide Web . 4-12-2012. Wall Street Journal. 
107. B. Lynn, personal communication, 2010.

108. Tomlinson WJ (1977) Appl. Opt. 16:2180-2194.

109. Leito I, Herodes K, Huopolainen M, Virro K, Kunnapas A, Kruve A, and Tanner R (2008) Rapid Commun. Mass Sp. 22:379-384.

110. Holcapek M, Jirasko R, and Lisa M (2010) J. Chromatogr. A. 1217:3908-3921.

111. Puton J, Nousiainen M, and Sillanpaa M (2008) Talanta 76:978-987.

112. Cole RB and Zhu JH (1999) Rapid Commun. Mass Sp. 13:607-611.

113. Cai Y and Cole RB (2002) Anal. Chem. 74:985-991.

114. Song LG, Wellman AD, Yao HF, and Bartmess JE (2007) Journal of the American Society for Mass Spectrometry 18:1789-1798.

115. Dillard JG (2002) Chem. Rev. 73:589-643.

116. Holness H and Almirall J (2013) Int. J. Ion Mobil. Spec.1-10.

117. Pirkle WH and Pochapsky TC (1989) Chem. Rev. 89:347-362.

118. Easson LH and Stedman E (1933) Biochemical Journal 27:1257-1266.

119. M. J. Fritsch, G. Trucks, J. Cheeseman, G. Scalmani, F. Clemente, H. Hratchian, M. Caricato, A. Izmaylov, J. Hess, A. C, A. C, M. G, K. T, J. T, J. C, D. Fox, K. Morokuma, I. Jacokowski, V. Ortiz, S. Zakrzewski, O. Dolgounitcheva, K. Raghavachari, G. Ferguson, N. Mayhall, O. Farkas, B. Schlegel, A. Birkholz, G. Scuseria, C. Diaconu, T. Henderson, J. Paier, C. Jiminez-Hoyos, A. Rusakov, T. Tsuchimochi, H. Nakatsuji, M. Robb, M. Bearpark, G. Petersson, J. Gao, F. Ogliaro, N. Rega, M. Ehara, C. Adamo, R. Cammi, B. Mennucci, A. Biancardi, S. Caprasecca, L. Viani, F. Lipparini, J. Tomasi, V. Barone, X. Li, J. Sonnenberg, and J. Foresman. GAUSSIAN 09. [Revision C.3.]. 2009. Pittsburgh, PA.

120. Becke AD (1993) Journal of Chemical Physics 98:5648-5652.

121. Lee CT, Yang WT, and Parr RG (1988) Physical Review B 37:785-789.

122. Pople JA, Headgordon M, and Raghavachari K (1987) Journal of Chemical Physics 87:5968-5975.

123. Jamal A and Mebel AM (2010) Physical Chemistry Chemical Physics 12:26062618. 
124. Jones BM, Zhang FT, Kaiser RI, Jamal A, Mebel AM, Cordiner MA, and Charnley SB (2011) Proceedings of the National Academy of Sciences of the United States of America 108:452-457.

125. Berthod A (2010) Chiral Recognition Mechanisms in Enantiomers Separations: A General View. In: Chiral Recognition in Separation Methods. Springer, New York.

126. Wu C, Siems WF, Klasmeier J, and Hill HH (2000) Anal. Chem. 72:391-395.

127. Enders JR and Mclean JA (2010) Chirality 21:E253-E264.

128. Howdle MD, Eckers C, Laures AMF, and Creaser CS (2010) International Journal of Mass Spectrometry 298:72-77.

129. (2011) CRC handbook of chemistry and physics, version 2012. Book News, Inc.

130. Allen A and Cantrell TS (1989) Forensic Sci int 42:183-199.

131. Aasim WRW, Gan SH, and Tan SC (2008) Biomed. Chromatogra. 22:1035-1042.

132. Inoue H, Iwata YT, and Kuwayama K (2008) J. Health Sci. 54:615-622.

133. Del Rio A (2009) J. Sep. Sci. 32:1566-1584.

134. Nassar AEF, Talaat RE, and Kamel AM (2006) Curr. Opin. Drug Disc. 9:61-74.

135. Tagliaro F, Manetto G, Bellini S, Scarcella D, Smith FP, and Marigo M (1998) Electrophoresis 19:42-50.

136. Mol R, de Jong GJ, and Somsen GW (2008) Rapid Commun. Mass Sp. 22:790796.

137. Mol R, Servais AC, Fillet M, Crommen J, de Jong GJ, and Somsen GW (2007) J. Chromatogr. A. 1159:51-57.

138. Nielen MWF (1993) Anal. Chem. 65:885-893.

139. Schug KA and Lindner W (2005) J. Sep. Sci. 28:1932-1955.

140. Shamsi SA (2001) Anal. Chem. 73:5103-5108.

141. Wu LM and Cooks G (2005) Eur. J. Mass Spectrom. 11:231-242.

142. Timothy J.Ward and Beth Ann Baker (2008) Anal. Chem. 80:4363-4372.

143. Fanali S (1996) J. Chromatogr. A. 735:77-121. 
144. Lai H, Guerra P, Joshi M, and Almirall JR (2008) J. Sep. Sci. 31:402-412.

145. Buryakov IA (2011) Journal of Analytical Chemistry 66:674-694.

146. Oxley JC, Smith JL, Kirschenbaum LJ, Marimganti S, and Vadlamannati S (2008) Journal of Forensic Sciences 53:690-693.

147. Joshi M, Delgado Y, Guerra P, Lai H, and Almirall JR (2009) Forensic Sci int 188:112-118.

148. Fetterolf DD and Clark TD (1993) Journal of Forensic Sciences 38:28-39.

149. Riikka-Marjaana Rasanen, Marjaana Nousiainen, Kaleva Perakorpi, Mika Sillanpaa, Lauri Polari, Osmo Anttalainen, and Mikko Utriainen (2008) Analytica Chimica Acta 673:59-65.

150. Guerra-Diaz P, Gura S, and Almirall JR (2010) Anal. Chem. 82:2826-2835.

151. Hulya Koyuncu, Ertan Seven, and Ayla Calimli (4 A.D.) Turk Journal of Chem 29:255-264.

152. Fuche C and Deseille J (2010) Actualite Chimique91-95.

153. Mitch Jacoby. Keepers of the Gate. Chemical and Engineering News 87[22], 1013. 6-1-2009. 6-17-2009.

154. Associated Press. Pressure cooker bombs suspected in Boston Blast. 4-17-2013. Boston Herald. 4-19-2013.

155. Spangler GE and Collins CI (1975) Anal. Chem. 47:393-402.

156. Carr TW (1977) Anal. Chem. 49:828-831.

157. Mathis JA and Mccord BR (2003) J. Chromatogr. A. 988:107-116.

158. Royds D, Lewis SW, and Taylor AM (2005) Talanta 67:262-268.

159. Thomas JL, Lincoln D, and Mccord BR (2013) Journal of Forensic Sciences 58:609-615.

160. Kabir A, Holness H, Furton KG, and Almirall JR (2012) 1.23 - Legal and Forensic Sampling. In: Comprehensive Sampling and Sample Preparation. Academic Press, Oxford.

161. Burks RM and Hage DS (2009) Analytical and Bioanalytical Chemistry 395:301313. 
162. Tam M and Hill HH (2004) Anal. Chem. 76:2741-2747.

163. Armitt D, Zimmermann P, and Ellis-Steinborner S (2008) Rapid Commun. Mass Sp. 22:950-958.

164. Faina Dubnikova, Ronnie Kosloff, Joseph Almog, Yehuda Zeiri, Roland Boese, Harel Itzhaky, Aaron Alt, and Ehud Keinan (2005) Journal of American Chemical Society 127:1146-1159.

165. Shen CY, Li JQ, Xu GH, Wang HM, Han HY, Zheng PC, Li H, Wang YJ, and Chu YN (2009) Chemical Journal of Chinese Universities-Chinese 30:274-278.

166. Matyas R and Selesovsky J (2009) Journal of Hazardous Materials 165:95-99.

167. Matyas R, Zeman S, Trzcinski W, and Cudzilo S (2008) Propellants Explosives Pyrotechnics 33:296-300.

168. C. E. Schumer. In the wake of Zazi bombing plot in NYC, Schumer urges justice department and DHS to crackdown on easily purchased chemicals that terrorists can turn into explosives . Internet source, updated 10-20-2010.accessed 2-242010

169. Nicholas A.Milas and Aleksander Golubovic (1959) American Chemical Society.

170. Times Online. TATP is suicide bomber's weapon of choice. Internet . 7-15-2005. 2-24-2010.

171. British Parliment. Intelligence and Security Committee Report into the London Terrorist Attacks on 7 July 2005. 1-45. 2006.

172. Johns C, Hutchinson JP, Breadmore MC, Guijt RM, Hilder EF, Dicinoski GW, and Haddad PR (2009) Chemistry in Australia 76.

173. Schulte-Ladbeck R, Kolla P, and Karst U (2003) Anal. Chem. 75:731-735.

174. Sigman ME, Clark CD, Caiano T, and Mullen R (2008) Rapid Commun. Mass Sp. 22:84-90.

175. Nicola Nugent. Breakthrough in trace detection of peroxide explosives. Chemical Science . 2-13-0006.

176. Ewing RC, Waltman MJ, and Atkinson DA (2011) Anal. Chem. 83:4838-4844.

177. Sigman ME, Clark CD, Fidler R, Geiger CL, and Clausen CA (2006) Rapid Commun. Mass Sp. 20:2851-2857.

178. Cotte-Rodriguez I, Chen H, and Cooks RG (2006) Chem. Commun.953-955. 
179. Ferguson CN, Benchaar SA, Miao ZX, Loo JA, and Chen H (2011) Anal. Chem. 83:6468-6473.

180. Harper RJ and Furton KG (2007) Biological Detection of Explosives. In: Counterterrorist Detection Techniques of Explosives. Elsevier.

181. Michael Krausa and Aleksey Alekseyvitch Reznev (2004) Vapor Detection of Explosives for Counter Terrorism. Kluwer Academic Publishers.

182. Jeannette M Perr, Kenneth G Furton, and Jose R Almirall (2005) J. Sep. Sci. 28:177-183.

183. J. M. Johnston. Canine Detection Capabilities: Operational Implications of Recent R \& D Findings. 1-7. 1999. Auburn University, Institute for Biological Detection Systems. 
VITA

Howard K Holness

Born Kingston, Jamaica

\section{EDUCATION}

$1999-2002$

Bachelor of Science, Applied and Food Chemistry

University of the West Indies

Mona, Jamaica

2004-2005

Master of Business Administration

University of New Orleans

New Orleans, Louisiana

$2008-2013$

Doctoral Candidate in Chemistry

Florida International University

Miami, Florida

\section{PUBLICATIONS:}

1. Holness, H.; Jamal, A.; Mebel, A.; Almirall, J. Separation mechanism of chiral impurities, ephedrine and pseudoephedrine, found in amphetaminetype substances using achiral modifiers in the gas phase. Analytical and Bioanalytical Chemistry 2012, 404 (8), 2407-2416.

2. Kabir, A.; Holness, H.; Furton, K. G.; Almirall, J. R. 1.23 - Legal and Forensic Sampling. In Comprehensive Sampling and Sample Preparation, Editor-in-Chief: Janusz Pawliszyn, Ed.; Academic Press: Oxford, 2012; pp 441-465.

3. Kabir, A.; Holness, H.; Furton, K. G.; Almirall, J. R. Recent advances in micro-sample preparation with forensic applications. TrAC Trends in Analytical Chemistry 2013, 45 (0), 264-279.

4. Holness, H.; Almirall, J. Speciation effects of solvent chemistry on the analysis of drugs and explosives by electrospray ion mobility mass spectrometry. Int. J. Ion Mobil. Spec. 2013, 16 (3), 237-246. 


\section{SELECTED PRESENTATIONS:}

1. Howard K. Holness and Jose R. Almirall, Fast Forensic Analysis of Methamphetamine and its Enantiomers Using Ion Mobility Spectrometry, Presented at the $62^{\text {nd }}$ American Academy of Forensic Sciences, Seattle, Washington, 2010

2. Kenneth G. Furton, Jessie Greb and Howard Holness, Improved Location of Forensic Evidence Through Implementation of the Scientific Working Group on Dog and Orthogonal Detector Guidelines (SWGDOG), Presented at the $62^{\text {nd }}$ American Academy of Forensic Sciences, Seattle, Washington, 2010

3. Howard K. Holness and José R. Almirall, SEPARATION OF ENANTIOMERS BY CHIRAL ION MOBILITY SPECTROMETRY, Presented at the Florida Annual Meeting and Exposition (FAME), Tampa, Florida, 2010

4. Mimy Young, Wen Fan, Howard Holness and Jose Almirall, Development of Stimulants of Hydrogen Peroxide Based Explosives for use by Canine and IMS Detectors, Presented at the Awareness and Localization of Explosives-Related Threats (ALERT) Site Visit, Boston, Massachusetts, 2011

5. Howard Holness and Jose Almirall, Chiral Separation of Amphetamine Type Substances Using Ion Mobility Spectroscopy - Mass Spectrometry, Presented at the $64^{\text {th }}$ American Academy of Forensic Sciences, Atlanta Georgia, 2012

6. Howard Holness, Seongshin Gwak and Jose Almirall, Chiral Separation of Amphetamine Type Substances Using Ion Mobility - Mass Spectrometry, Presented at $1^{\text {st }}$ IFRI Forensic Science Symposium, Miami, Florida 2012

7. Nicholas Weis, Howard Holness and José Almirall, METHOD OPTIMIZATION FOR THE ANALYSIS OF SMOKELESS POWDERS BY ELECTROSPRAY IONIZATION-ION MOBILITY-MASS SPECTROMETRY, Presented at the Florida Annual Meeting and Exposition (FAME), Tampa Florida 2012.

8. Stephanie Luquis, Howard Holness and José Almirall, Analysis of Smokeless Powder by Gas Chromatography-Mass Spectrometry and Ion Mobility Mass Spectrometry, Presented at Florida International University, Research Experience for Undergraduates (REU), 2013 